University of Tennessee Health Science Center

UTHSC Digital Commons

Theses and Dissertations (ETD)

College of Graduate Health Sciences

$12-2013$

\title{
ERG11-Mediated Azole Resistance in Candida albicans
}

Stephanie Ann Flowers

University of Tennessee Health Science Center

Follow this and additional works at: https://dc.uthsc.edu/dissertations

Part of the Bacterial Infections and Mycoses Commons, Fungi Commons, Medical Immunology Commons, Medicinal and Pharmaceutical Chemistry Commons, and the Pharmaceutical Preparations Commons

\section{Recommended Citation}

Flowers, Stephanie Ann, "ERG11-Mediated Azole Resistance in Candida albicans" (2013). Theses and Dissertations (ETD). Paper 335. http://dx.doi.org/10.21007/etd.cghs.2013.0096.

This Dissertation is brought to you for free and open access by the College of Graduate Health Sciences at UTHSC Digital Commons. It has been accepted for inclusion in Theses and Dissertations (ETD) by an authorized administrator of UTHSC Digital Commons. For more information, please contact jwelch30@uthsc.edu. 


\title{
ERG11-Mediated Azole Resistance in Candida albicans
}

\begin{abstract}
Although many medically important Candida species are commensal to the gut or colonizers of the skin, these organisms have the propensity to cause disease in the event of a waning immune system. Clinical manifestations of infections with Candida species can range from superficial mucosal infections to deep organ involvement usually resulting from haematogenous spread of infection. Despite significant progress made in the management of patients with fungal infections, the emergence of antifungal resistant clinical isolates creates significant problem in regards to antifungal prophylaxis and empirical treatment strategies. Antifungal resistance is associated with high mortality rates and hefty medical costs. The azole-antifungal class has been the "work horse" of antifungal pharmacotherapy for the past 20 years defined by its efficacy against Candida species and paucity of side effects. As the only oral option available for systemic antifungal treatment, the azoles are the most suitable option for the long treatment periods sometimes required for antifungal prophylaxis and therapy. As the azoles are fungistatic to Candida species, the lengthy and repeated treatment courses have resulted in azoleresistant clinical isolates resulting in treatment failure and increased patient mortality.
\end{abstract}

Candida albicans is the most prevalent etiologic cause of fungal disease. High-level azole resistance in this species is a result of the interplay of several mechanisms of resistance. Overexpression of the efflux transporter genes $C D R 1, C D R 2$, and MDR1 is a common mechanism of drug resistance in $C$. albicans and the majority of previous investigations pertained to defining mechanisms of transcriptional regulation of efflux transporters. Alternatively to efflux transport, point mutations in the ERG11 gene, whose gene product is the target of azoles, result in reduced target binding affinity without precluding enzymatic function. In addition to point mutations, overexpression of $E R G 11$ has also been shown to decrease fluconazole susceptibility. ERG11 gene amplification by chromosome 5 duplication or the presence of a chr5L isochromosome is known to contribute to azole resistance. Additionally, the zinc-cluster transcription factor Upc2 has been shown to regulate the expression of ERG11 and other genes involved in ergosterol biosynthesis.

In a large group of clinical $C$. albicans isolates enriched for azole resistance, I created a transcriptional profile defining expression of genes known to cause azole resistance such as ERG11,CDR1,CDR2 and MDR1. Not surprisingly, CDR1 and CDR2 overexpression was generally coordinately regulated and quite prevalent among these isolates. Of those isolates that did overexpress MDR1, even fewer isolates expressed MDR1 to the levels previously observed in azoleresistant isolates. ERG11 was found to be upregulated in almost three-fourths of the fluconazoleresistant isolates examined. This suggests that $E R G 11$ overexpression is a common contributor to fluconazole resistance in $C$. albicans. Among the ERG11-overexpressing isolates studied here, I repeatedly recovered eight distinct single-nucleotide substitutions in UPC2. Five of these substitutions in UPC2 have not been described previously. Of the five novel mutations, four mutations resulted in increased ERG11 expression and increased resistance to fluconazole but to various degrees. Genome-wide transcriptional analysis was performed for the four strongest Upc2 amino acid substitutions (A643V, G648D, G648S, and Y642F). Genes commonly upregulated by all four mutations included those involved in ergosterol biosynthesis, in oxidoreductase activity, the major facilitator efflux pump encoded by the MDR1 gene, and the uncharacterized ATP binding cassette transporter CDR11. These findings demonstrate that gain-of-function mutations in UPC2 are more prevalent among clinical isolates than previously thought and make a significant contribution to azole antifungal resistance, but the findings do not account for ERG11 overexpression in all such isolates of $C$. albicans.

Although prevalent, not all ERG11-overexpression in this group of isolates could be explained by GOF mutations in Upc2. In C. albicans, the Pho-G transcription factor NDT80 has been implicated in azole 
resistance not only due to its regulation of $C D R 1$ but also due to its regulation of genes involved in the ergosterol biosynthesis pathway. In the next set of experiments, NDT80 alleles for genetically matched pairs of isolates $945 / 1619$ and 1002/3795 were sequenced. In both matched sets, the fluconazole resistant isolate overexpresses ERG11. Sequencing of the NDT80 allele of both matched sets revealed several mutations that resulted in amino acid substitutions when compared to SC5314. This analysis also showed that a loss of heterozygostiy event occurs so that the resistant counterpart was homozygous for one allele. A strain carrying the NDT80 allele derived from fluconazole-resistant isolate 1619 did not result in increased ERG11 expression and increased fluconazole resistance. The mechanism by which ERG11 is upregulated in the absence of UPC2 gain-of-function mutations is currently under investigation.

In addition to ERG11-overexpression, mutations in ERG11 that result in amino acid substitutions in lanosterol demethylase have also been associated with decreased azole susceptibility. In the third study, I examined the prevalence and variance of ERG11 mutations in the same group clinical $C$. albicans isolates. In this collection, I identified that 55 of the 63 isolated contained missense mutations in ERG11 that resulted in at least one amino acid substitution. From this sequencing data, a selected a group of mutant ERG11 alleles were expressed in an azole-susceptible background so I could determine the specific contribution of the mutant ERG11 allele on antifungal susceptibility. In this analysis, I was particularly interested in characterizing amino acid substitutions that occurred alone and also when accompanied by another mutation. In total, I characterized ten ERG11 alleles containing one amino acid substitution and nine alleles which carried ERG11 alleles with a combination of amino acid substitutions. Fluconazole, itraconazole and voriconazole susceptibilities for these strains were tested. Our data demonstrated many of these mutations resulted in fluconazole resistance, but most were not as significant when tested against voriconazole or itraconazole. Itraconazole, in particular seemed less effected by ERG11 mutations which produced significant resistance to fluconazole although amino acid combination $\mathrm{Y} 132 \mathrm{~F}$ and $\mathrm{F} 145 \mathrm{~L}$ resulted in increased itraconazole resistance. Specific combinations of ERG11 mutations resulted in increased azole resistance beyond single mutations. These data suggest that structural differences between azole effect activity against specific mutant ERG11 alleles.

\section{Document Type}

Dissertation

\section{Degree Name}

Doctor of Philosophy (PhD)

\section{Program}

Biomedical Sciences

\section{Research Advisor}

P. David Rogers, Pharm.D., Ph.D.

\section{Keywords}

albicans, azole, Candida, fluconazole, resistance

\section{Subject Categories}

Bacterial Infections and Mycoses | Chemicals and Drugs | Diseases | Fungi | Medical Immunology | Medical Sciences | Medicinal and Pharmaceutical Chemistry | Medicine and Health Sciences | Organisms | Pharmaceutical Preparations | Pharmacy and Pharmaceutical Sciences

\section{Comments}

Two year embargo expired December 2015

This dissertation is available at UTHSC Digital Commons: https://dc.uthsc.edu/dissertations/335 


\title{
ERG11-Mediated Azole Resistance in Candida albicans
}

\author{
A Dissertation \\ Presented for \\ The Graduate Studies Council \\ The University of Tennessee \\ Health Science Center
}

In Partial Fulfillment

Of the Requirements for the Degree

Doctor of Philosophy

From The University of Tennessee

By

Stephanie Ann Flowers

December 2013 
Chapter 2 (C) 2012 by American Society of Microbiology. All other material (C) 2013 by Stephanie A. Flowers.

All rights reserved. 


\section{DEDICATION}

To my parents, Marcia Flowers and Allan Flowers, and to my step-parents, Roger Alaux and Jacqueline Flowers, for all their love and support. To my grandmother and "grandfather", Virginia Clarine Walton and Burton Zoul, you are greatly missed. 


\section{ACKNOWLEDGEMENTS}

First I would like express my sincere gratitude to my advisor, Dave Rogers, for giving me the opportunity to work in his lab and for facilitating professional relationships with researchers and academic pharmacists. Thank you for the encouragement provided throughout this process and the investment made in my future. I would also like to thank my committee members, Richard Lee, Ramin Homayouni, Bernd Meibohm and Todd Reynolds for their support and guidance. Thank you for the thoughtful and critical review of my dissertation.

A special thanks to Tom Cunningham at the Molecular Resource Center for his help with the large amount of sequencing work that made this project possible. To my friends Young-In Kim- and Chris Hoehamer, Juanita White and Clare Wimbiscus for keeping life balanced.

Last, but certainly not least, I would like to thank the members of the Rogers lab, Kathy Barker, Kelly Caudle, Teresa Liu, Erin Vasicek, Sarah Whaley, Andy Nishimoto, Beth Berkow and Kayihura Manigaba. To my partner in crime Qing Zhang, this wouldn't be possible without your help. 


\begin{abstract}
Although many medically important Candida species are commensal to the gut or colonizers of the skin, these organisms have the propensity to cause disease in the event of a waning immune system Clinical manifestations of infections with Candida species can range from superficial mucosal infections to deep organ involvement usually resulting from haematogenous spread of infection. Despite significant progress made in the management of patients with fungal infections, the emergence of antifungal resistant clinical isolates creates significant problem in regards to antifungal prophylaxis and empirical treatment strategies. Antifungal resistance is associated with high mortality rates and hefty medical costs. The azole-antifungal class has been the "work horse" of antifungal pharmacotherapy for the past 20 years defined by its efficacy against Candida species and paucity of side effects. As the only oral option available for systemic antifungal treatment, the azoles are the most suitable option for the long treatment periods sometimes required for antifungal prophylaxis and therapy. As the azoles are fungistatic to Candida species, the lengthy and repeated treatment courses have resulted in azoleresistant clinical isolates resulting in treatment failure and increased patient mortality.
\end{abstract}

Candida albicans is the most prevalent etiologic cause of fungal disease. High-level azole resistance in this species is a result of the interplay of several mechanisms of resistance. Overexpression of the efflux transporter genes $C D R 1, C D R 2$, and $M D R 1$ is a common mechanism of drug resistance in $C$. albicans and the majority of previous investigations pertained to defining mechanisms of transcriptional regulation of efflux transporters. Alternatively to efflux transport, point mutations in the ERG11 gene, whose gene product is the target of azoles, result in reduced target binding affinity without precluding enzymatic function. In addition to point mutations, overexpression of ERG11 has also been shown to decrease fluconazole susceptibility. ERG11 gene amplification by chromosome 5 duplication or the presence of a chr5L isochromosome is known to contribute to azole resistance. Additionally, the zinc-cluster transcription factor Upc2 has been shown to regulate the expression of ERG11 and other genes involved in ergosterol biosynthesis.

In a large group of clinical $C$. albicans isolates enriched for azole resistance, I created a transcriptional profile defining expression of genes known to cause azole resistance such as ERG11,CDR1,CDR2 and MDR1. Not surprisingly, CDR1 and CDR2 overexpression was generally coordinately regulated and quite prevalent among these isolates. Of those isolates that did overexpress $M D R 1$, even fewer isolates expressed $M D R 1$ to the levels previously observed in azole-resistant isolates. ERG11 was found to be upregulated in almost three-fourths of the fluconazole-resistant isolates examined. This suggests that ERG11 overexpression is a common contributor to fluconazole resistance in C. albicans. Among the ERG11-overexpressing isolates studied here, I repeatedly recovered eight distinct single-nucleotide substitutions in UPC2. Five of these substitutions in $U P C 2$ have not been described previously. Of the five novel mutations, four mutations resulted in increased ERG11 expression and increased resistance to fluconazole but to various degrees. Genome-wide transcriptional analysis was performed 
for the four strongest Upc2 amino acid substitutions (A643V, G648D, G648S, and Y642F). Genes commonly upregulated by all four mutations included those involved in ergosterol biosynthesis, in oxidoreductase activity, the major facilitator efflux pump encoded by the MDR1 gene, and the uncharacterized ATP binding cassette transporter $C D R 11$. These findings demonstrate that gain-of-function mutations in UPC2 are more prevalent among clinical isolates than previously thought and make a significant contribution to azole antifungal resistance, but the findings do not account for ERG11 overexpression in all such isolates of $C$. albicans.

Although prevalent, not all ERG11-overexpression in this group of isolates could be explained by GOF mutations in Upc2. In C. albicans, the Pho-G transcription factor NDT80 has been implicated in azole resistance not only due to its regulation of CDR1 but also due to its regulation of genes involved in the ergosterol biosynthesis pathway. In the next set of experiments, NDT80 alleles for genetically matched pairs of isolates $945 / 1619$ and 1002/3795 were sequenced. In both matched sets, the fluconazole resistant isolate overexpresses ERG11. Sequencing of the NDT80 allele of both matched sets revealed several mutations that resulted in amino acid substitutions when compared to SC5314. This analysis also showed that a loss of heterozygostiy event occurs so that the resistant counterpart was homozygous for one allele. A strain carrying the NDT80 allele derived from fluconazole-resistant isolate 1619 did not result in increased ERG11 expression and increased fluconazole resistance. The mechanism by which $E R G 11$ is upregulated in the absence of UPC2 gain-of-function mutations is currently under investigation.

In addition to ERG11-overexpression, mutations in ERG11 that result in amino acid substitutions in lanosterol demethylase have also been associated with decreased azole susceptibility. In the third study, I examined the prevalence and variance of ERG11 mutations in the same group clinical $C$. albicans isolates. In this collection, I identified that 55 of the 63 isolated contained missense mutations in ERG11 that resulted in at least one amino acid substitution. From this sequencing data, a selected a group of mutant ERG11 alleles were expressed in an azole-susceptible background so I could determine the specific contribution of the mutant $E R G 11$ allele on antifungal susceptibility. In this analysis, I was particularly interested in characterizing amino acid substitutions that occurred alone and also when accompanied by another mutation. In total, I characterized ten ERG11 alleles containing one amino acid substitution and nine alleles which carried ERG11 alleles with a combination of amino acid substitutions. Fluconazole, itraconazole and voriconazole susceptibilities for these strains were tested. Our data demonstrated many of these mutations resulted in fluconazole resistance, but most were not as significant when tested against voriconazole or itraconazole. Itraconazole, in particular seemed less effected by ERG11 mutations which produced significant resistance to fluconazole although amino acid combination Y132F and F145L resulted in increased itraconazole resistance. Specific combinations of ERG11 mutations resulted in increased azole resistance beyond single mutations. These data suggest that structural differences between azole effect activity against specific mutant ERG11 alleles. 


\section{TABLE OF CONTENTS}

CHAPTER 1. INTRODUCTION ......................................................................................1

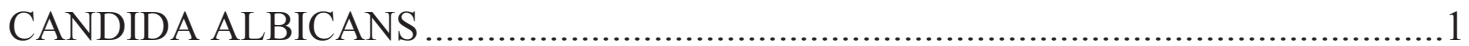

The historical importance of fungus and fungal pathogens .....................................

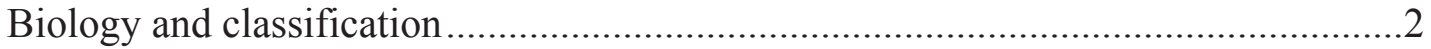

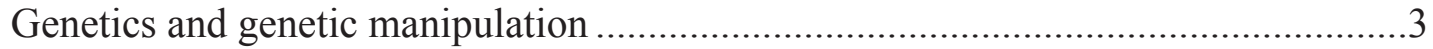

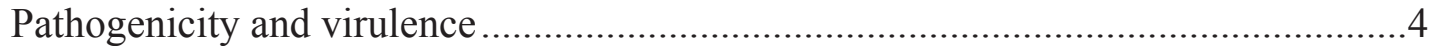

Infections caused by Candida species .............................................................6

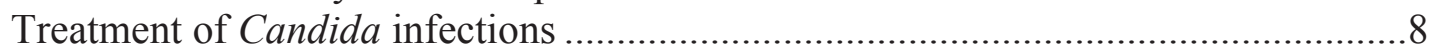

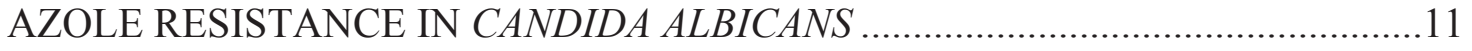

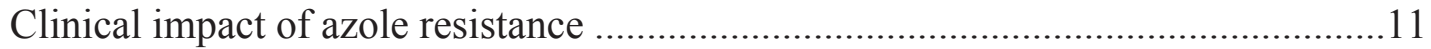

Molecular mechanisms of azole resistance in $C$. albicans ......................................12

TRANSCRIPTIONAL REGULATORS OF AZOLE RESISTANCE........................14

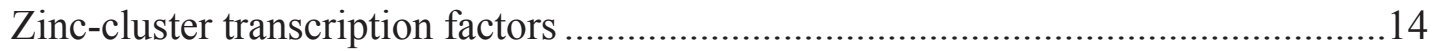

Zinc-cluster transcription factors and azole resistance in $C$. albicans ......................15

CENTRAL HYPOTHESIS AND RESEARCH OBJECTIVES ...............................17

\section{CHAPTER 2. GAIN-OF-FUNCTION MUTATIONS IN UPC2 ARE A} FREQUENT CAUSE OF ERG11 UPREGULATION IN AZOLE-RESISTANT

CLINICAL ISOLATES OF CANDIDA ALBICANS...................................................19

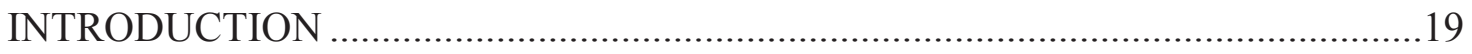

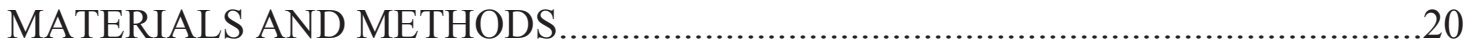

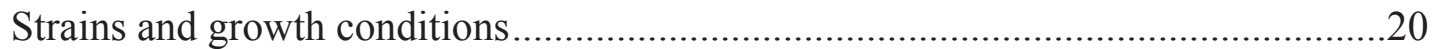

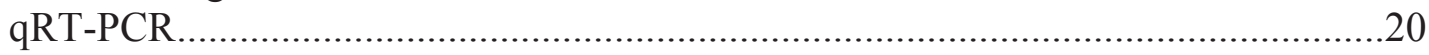

Plasmid construction for allele sequencing.............................................................20

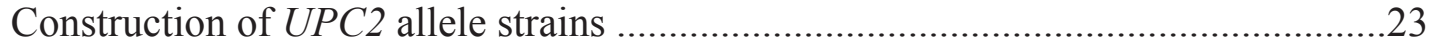

Fluconazole susceptibility testing. ......................................................................23

Ergosterol quantification analysis .....................................................................24

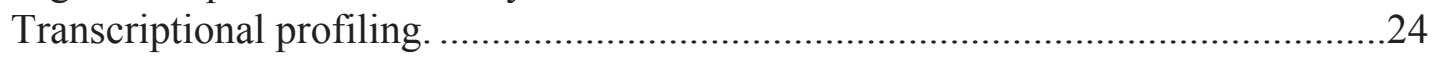

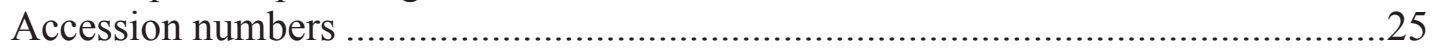

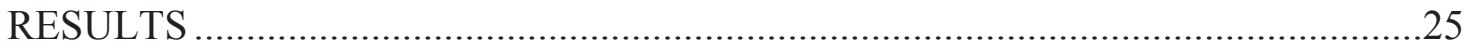

Expression of ERG1 1, CDR1, CDR2, and MDR1 in 62 fluconazole-resistant

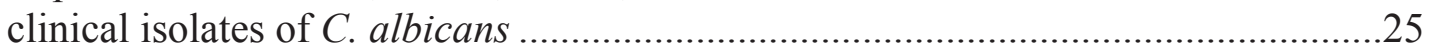

Most but not all isolates that overexpress ERG11 carry a GOF mutation in UPC2..25 GOF mutations in UPC2 result in increased expression of ERG11 ….....................28

GOF mutations inUPC2 result in elevated cellular ergosterol content......................28

GOF mutations in UPC2 influence susceptibility to antifungals...............................28

Genome-wide transcriptional-profile analysis of strains carrying activated UPC2

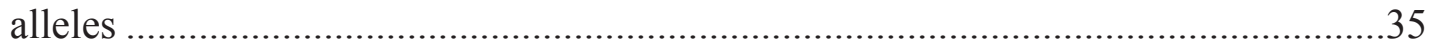

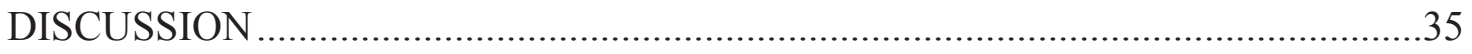

CHAPTER 3. NDT80 MUTATIONS FOUND IN CLINICAL ISOLATES OF CANDIDA ALBICANS DO NOT CONTRIBUTE TO AZOLE RESISTANCE BY

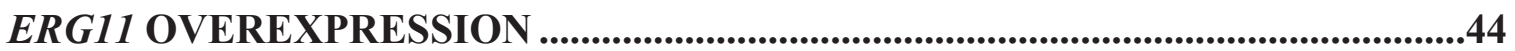




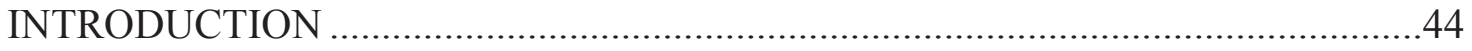

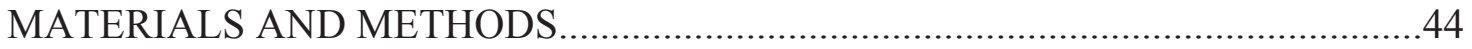

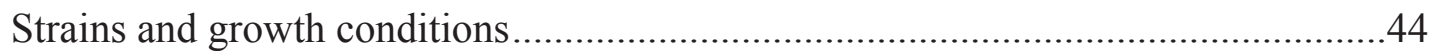

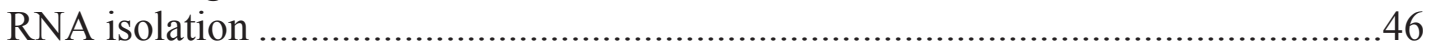

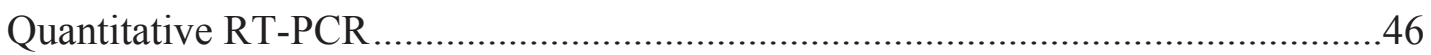

Plasmid construction for allele sequencing ...........................................................46

Construction of NDT80 allele strains...............................................................48

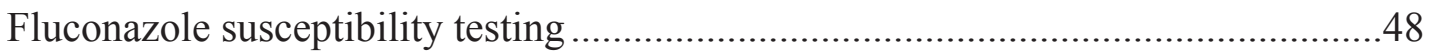

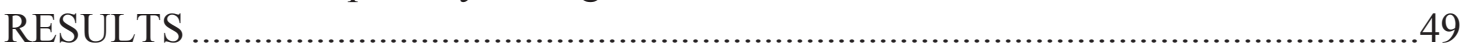

Sequencing NDT80 of matched isolate sets $945 / 1619$ and 1002/3795 revealed multiple single nucleotide polymorphisms and a loss of heterozygosity event in the NDT80 alleles of the resistant isolate.

Mutations observed in NDT80 of resistant isolates do not result in fluconazole

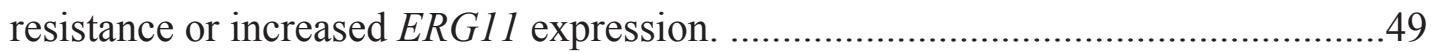

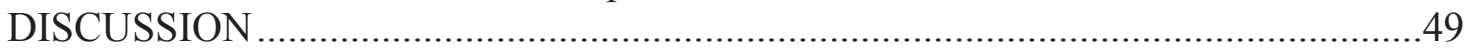

\section{CHAPTER 4. CLINICALLY DERIVED MUTATIONS IN ERG11} CONTRIBUTE TO AZOLE RESISTANCE IN CANDIDA ALBICANS...................52

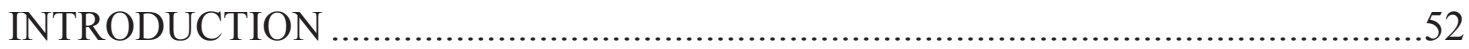

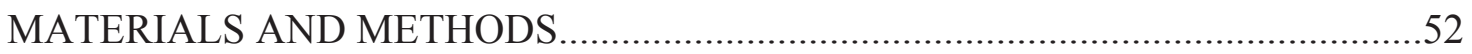

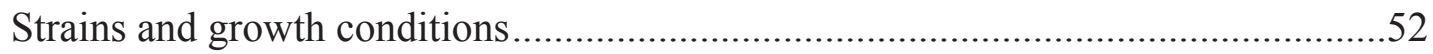

Plasmid construction for allele sequencing.............................................................53

Construction of strains carrying specific ERG11 alleles .........................................53

Azole susceptibility testing ..................................................................................57

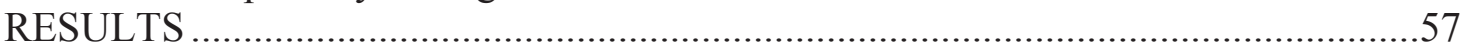

Many clinical isolates with reduced fluconazole susceptibility carry mutations in

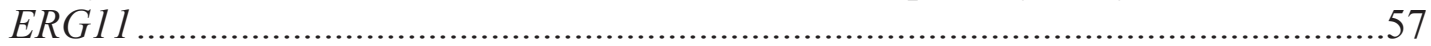

Single mutations in ERG11 contribute to azole resistance in C. albicans.................57

Multiple mutations in ERG11 can result in decreased azole susceptibility ...............63

Amino acid substitution E266D does not contribute to azole resistance ....................65

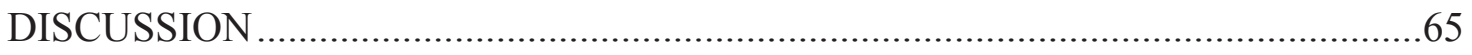

CHAPTER 5. DISCUSSION AND CONCLUSIONS .....................................................75

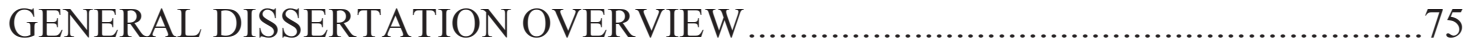

Summary and discussion of Chapter 3 ………………….....................................77

Summary and discussion of Chapter 4................................................................77

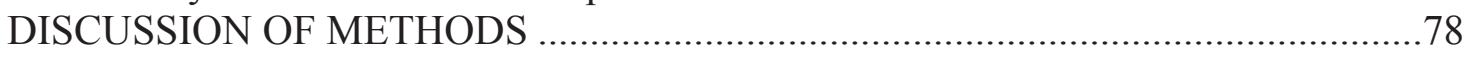

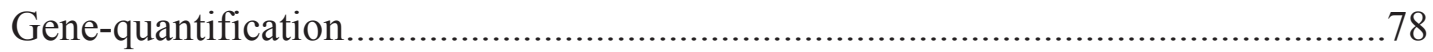

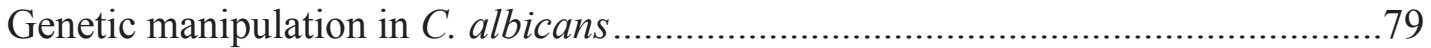

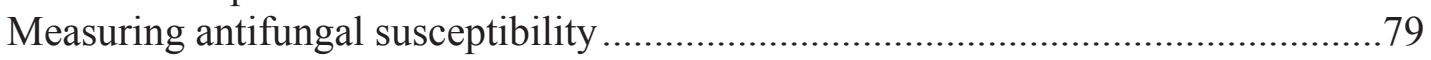

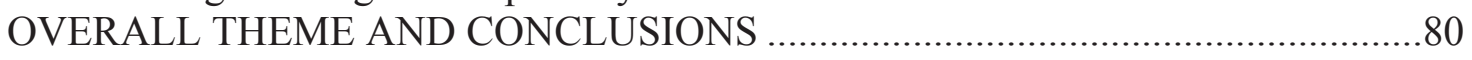

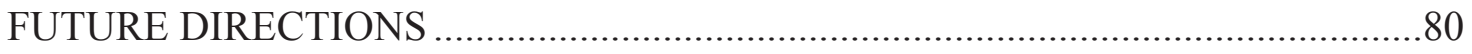

Whole genome sequencing to investigate $U P C$-independent mechanisms of

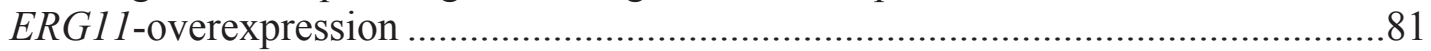

Employing genotypic testing as a tool to guide antifungal therapy ............................82 
LIST OF REFERENCES......................................................84

APPENDIX A. $C$. ALBICANS STRAINS TABLE.................................100 APPENDIX B. GENES UPREGULATED IN STRAINS CARRYING UPC2 GOF ALLELES BY AT LEAST 1.5 FOLD .....................................................104 APPENDIX C. GENES DOWNREGULATED IN STRAINS CARRYING UPC2 GOF ALLELES BY AT LEAST 1.5 FOLD.............................................131

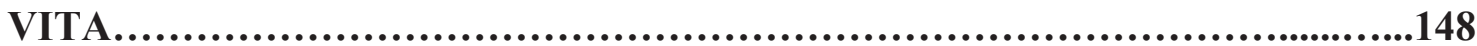




\section{LIST OF TABLES}

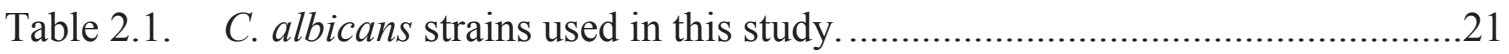

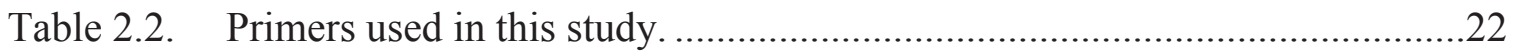

Table 2.3. Occurrence of Upc2 amino acid substitutions in predicted translated sequence in fluconazole resistant clinical ERG11-overexpressing isolates.

Table 2.4. MICs of strains expressing UPC2 mutant alleles to a panel of antifungals

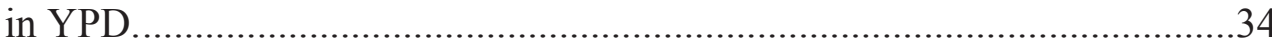

Table 2.5 Genes upregulated by at least 1.5- fold in strains carrying UPC2 GOF alleles

Table 2.6. Genes downregulated by at least 1.5-fold in strains carrying UPC2 GOF alleles.

Table 3.1. NDT80 strains and primers used in this study.

Table 3.2. NDT80 sequencing data from matched isolate sets 580, 2440 and 945, 1619 were compared to the SC5314.

Table 4.1 C. albicans strains carrying mutant ERG11 alleles used in this study .........54

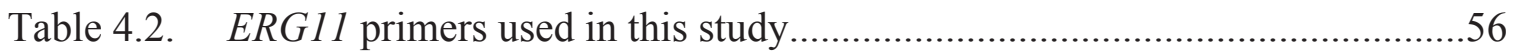

Table 4.3. Occurrence of Erg11 amino acid substitutions in predicted translated sequence in fluconazole resistant clinical ERG11-overexpressing isolates.

Table 4.4. Forty-eight hour MICs to FCZ, ICZ and VCZ measured by using a modified CLSI broth microdilution methods of $C$. albicans constructed to carry mutant ERG11 alleles homozygously. 


\section{LIST OF FIGURES}

Figure 2.1. Expression levels of $C D R 1, C D R 2, M D R 1$, and $E R G 11$ in 63

fluconazole-resistant clinical isolates

Figure 2.2. ERG11 transcription levels in strains carrying mutant UPC2 alleles are represented as averages from three independent qRT-PCR assays.

Figure 2.3. Ergosterol quantification in C. albicans laboratory strains expressing mutant UPC2 alleles.

Figure 3.1. ERG11 expression of strains constructed to carry NDT80 alleles derived from clinically matched isolates 945 and 1619

Figure 4.1. Cyp51 secondary structure is conserved despite low sequence homology between kingdoms

Figure 4.2. Sequence alignment highlighting the fungal specific major insertion loop in various fungal species and contrasted with the non-fungal species, $M$. tuberculosis.

Figure 4.3. Observed amino acid subsitutions in $\operatorname{Erg} 11$ in C. albicans compared to other medically important Candida species and organisms in other kingdoms. 


\section{CHAPTER 1. INTRODUCTION}

\section{CANDIDA ALBICANS}

\section{The historical importance of fungus and fungal pathogens}

As immunocompetent mammals are naturally resistant to fungus, infections by fungal organisms seem enigmatic and dangerous. Mammals' innate immunity to fungal infections is likely due to a combination of the innate and adaptive immune system in addition to elevated body temperatures characterized by our endothermic lifestyle (19). A computational model has determined the optimal temperature that provides maximal protection against fungus to be $36.7^{\circ} \mathrm{C}$, which is close to mammalian temperatures (11). This data suggests that vertebrate endothermic physiologic temperatures could be in part selected by pathogenic fungal species (19). An example of protection conferred by both a competent immune system and an endothermic physiological temperature has been demonstrated recently in the local bat population. In August of 2013, British Broadcasting Company reported a "bat killing fungus" detected in Arkansas that is responsible for the devastation of the North American bat population. The visual symptom of infected bats is a white fungus that typically grows on the nose, ears or tails, earning the title "white nose syndrome" (31). The etiologic cause of this infection is the fungus Pseudogymnoascus destructans. This fungus is unable to grow at temperatures above $20^{\circ} \mathrm{C}$ and infects bats as their body temperature lowers due to hibernation (30). Dr. Arturo Casadevall, from Albert Einstein School of Medicine has attributed mammalian immunity to disseminated fungal infections with the dominance of mammals after the Cretaceous-Tertiary (K-T) Boundary (19). In his article entitled "Fungi and the Rise of Mammals", Dr. Casadevall describes how the "fungal bloom" resulting from catastrophic events 65 million years ago set the stage for mammals replacing reptiles as the dominant land animals $(19,192)$.

Historically, the progress defining the significance of medically relevant mycology was slow due to the infrequency of disease and lack of experience of medical practitioners. The first observations of Candida infections is thought to date back to the ancient Greek physician Hippocrates who described an oral candidiasis, or thrush, around $400 \mathrm{BC}$ in book three in "Of the Epidemics" (10). In this selection, what is presumed to be an observation of oral candidiasis is described as "mouths affected with aphthous ulcerations". Much later in history but prior to Louis Pasteur, the Hungarian physician David Gruby identified the human pathogen, later named Achorion schoenleinii, to cause the scalp condition called favus (72). He was able to demonstrate causation of human disease by culturing the microorganism on potato slices and infecting healthy tissue.

Despite the early observation and detection of diseases caused by fungal pathogens, bacterial diseases are better studied. This is most likely due to the frequency and severity of bacterial and mycobacterial epidemics that affect large populations as opposed to infrequent and isolated encounters with fungal pathogens. The current accepted genus for 
C. albicans was introduced in 1923 by Christine Berkhout, who was later credited with deriving the first "rational systematics of anascosporogenous yeasts" (10). The genus Candida is derived from ancient Roman political candidates, or candidatus, who wore white togas. The species name albicans is derived from Albico, which also means "to be white" (10). Prior to the $1980 \mathrm{~s}$, mortality attributed to mycosis was ranked $10^{\text {th }}$ in the United States when compared to mortality rates from alternate infectious etiologies. Between the time periods of 1980 to 1997 , a marked increase of mortality attributed to fungal pathogens occurred, which is a time period that correlates with the onset of HIV and medical advancements that prolong the life of critically ill patients that are susceptible to these pathogens (118).

On June 5, 1981, the Centers for Disease Control and Prevention (CDC) published in their Morbidity and Mortality Weekly Report (MMWR) describing independent case studies of five homosexual, previously healthy young men presenting with what was then known as Pneumocystis carinii, which is an opportunistic pathogen. (1). These men presented with other opportunistic infections and three cases noted oral candidiasis on their medical problem list. This publication is considered by many the beginning of the AIDs epidemic. With the spread of AIDs, detection of mucosal and invasive fungal opportunistic pathogens exploded (118). The most common pathogens in this population were Candida albicans and Cryptococcus neoformans (6). Mucocutaneous candidiasis was the most frequently identified fungal opportunistic infection and the most common early identifier of people with Human Immunodeficiency Virus (HIV)(118). With the implementation of Highly Active Antiretroviral Therapy (HAART), the frequency of Candida spp. infections in HIV patients has dramatically decreased (188). However, in populations that do not have access to antiretrovirals or in cases where patients are noncompliant or are non-responders to their HAART regimen, treatment courses of azole antifungals have resulted in a subset of isolates that have become resistant to treatment (188).

Despite the successful development of treatments for HIV, the population susceptible to invasive fungal infections continues to grow and Candida spp are leading the charge. Advances made in invasive medical technology, immunosuppressive and chemotoxic treatments have resulted in a wide range of subjects susceptible to invasive fungal disease. In nosocomial derived bloodstream infections, Candida spp the rank overall as the fourth most commonly isolated pathogen in hospitals and are identified as the second most frequent cause of infection in North American intensive care units $(198,205) . C$. albicans remains the most prevalent etiological agent of Candida infection.

\section{Biology and classification}

C. albicans is both a commensal organism and a medically important opportunistic pathogen. This species taxonomically belongs to the Fungi kingdom, the phylum Ascomycota, which is the largest phylum within the kingdom Fungi, and the class Saccharomycotina (87). The genus Candida is within the class of Deuteromycetes, since many species of this genus were not observed to have a sexual cycle. Candida spp. 
includes more than 160 species but only 20 of these are clinically relevant in human disease (10).

C. albicans is a diploid, polymorphic fungus that is member of the CTG clade defined by a unique codon exchange from a leucine to a serine (183). Its mode of reproduction is predominately clonal but still exhibits substantial heterozygosity due to its ability to undergo recombination and ploidy changes based on chromosomal loss and reformation (134). C. albicans possesses homologous of the mating-type genes that are characterized in Saccharomyces cerevisiae and can undergo a mating process which results in a tetraploid cell (76). C. albicans lack genes to undergo meiosis so return to the diploid state is a result of random chromosomal loss and is also a source of genomic microvariation in this species $(12,133)$. DNA fingerprinting techniques have been developed to identify population structure and microevolution in C. albicans. Results of these studies have defined five major genetically related groups, designated as clades, which are geographically linked to specific areas of the world (179).

\section{Genetics and genetic manipulation}

C. albicans generally is considered to be an obligate diploid organism but has been recently shown to have a viable haploid state that is recovered both in vitro and in vivo (70). C. albicans propagates predominantly by clonal methods however, a parasexual cycle has been defined for this species $(12,80)$. The nuclear genome is around 16 million base pairs comprised of eight chromosomes (82). The chromosomes are numbered from 1 $(\sim 4.3 \mathrm{Mb})$ to $7(\sim 1.03 \mathrm{Mb})$ and also chromosome $\mathrm{R}$, which varies in size depending on the number of ribosomal DNA repeats. The complete sequence of $C$. albicans is available on the Candida Genome Database (CGD) website from Stanford University (www.candidagenome.org).

The success of $C$. albicans as an opportunistic pathogen is due to its ability to adapt to various niches aided by genetic changes such as point mutations, loss of heterozygosity (LOH) events, and instances of aneuploidies (134). C. albicans has a high level of single nucleotide polymorphisms (SNPs), which cause amino acid changes in the protein sequence. Previous work has shown that out of the approximate 6,000 total genes of the C. albicans genome, about 2,792 open reading frames (ORFs) carry at least one SNP resulting in heterozygous alleles in almost half the genome (18).

Over the last few decades, molecular pathogenicity and drug resistance mechanisms in Candida species have been intensely investigated using genetic tools and molecular techniques which were often times developed to study genetics in $S$. cerevisiae. Creating libraries of gene disruptants has been a powerful tool in the examination genetic regulatory networks of specific cellular processes $(69,132)$. In C. albicans, homozygous gene disruption requires two rounds of transformation because it is a diploid species. By this method, a linear fragment of exogenous DNA is introduced with a selectable marker flanked by specific homologous regions which facilitates the targeted incorporation into the genome (69). This disruption cassette can be constructed by using polymerase chain 
reaction $(\mathrm{PCR})$ or traditional cloning techniques and selectable markers can be either auxotrophic genes ( $L E U 2, A R G 4$ and $U R A 3)$ or drug resistance genes such as SAT1 (156). Using $U R A 3$ auxotrophic strains have generally lost favor due to the observation that $U R A 3$ expression is correlated with virulence in this species (182).

The use of DNA mircroarrays has been the method of choice for examining genomewide gene transcription of $C$. albicans. Simply put, an array is a collection of single stranded DNA probes fixed on a solid surface. These probes can be deposited or synthesized in situ onto materials such as nitrocellulose, nylon or glass (56). Custom arrays for various species are now commercially available but variation can occur that affect gene expression levels such as labeling efficiency of specific dyes, concentration and quality of RNA starting material, and preparation of PCR products. Our lab has employed the use of microarrays to examine the differences in gene expression between fluconazole-susceptible and fluconazole-resistant strains in order to identify genes important for azole resistance $(44,50,158)$. Gene expression in azole resistance will be discussed in a later section.

Unlike bacteria who can acquire antimicrobial resistance by transposable elements, antifungal resistance in pathogenic fungi is primarily due to genetic variability such as single nucleotide polymorphisms (SNPs) (125). In bacteria, whole-genome sequencing techniques have provided insight into the development and transmission of antimicrobial resistant pathogens (64). In Candida spp. the acquisition of drug resistance has been studied by strains that have developed resistant mutations in vitro or by targeted sequencing and expression analysis of genes known to cause resistance in clinical matched-paired isolates (155). Developments in sequencing technology allows us to examine sequence on a genome-wide scale and permits the detection mutations that leads to drug resistance or even accompanying mutations that may mitigate fitness costs of resistance mutations. Recently, the first study to examine the genome-wide sequence analysis of echinocandin resistance in clinical isolates of $C$. glabrata was published (178). In this study authors were able to identify mutations in previously unidentified genes conferring resistance to echinocandins, but also identified a compensatory mutation that mitigated the fitness cost of echinocandin resistance.

\section{Pathogenicity and virulence}

C. albicans has the ability to cause a range of infectious from superficial mucosal or vaginal infections to disseminated disease. The ability of $C$. albicans to cause such a diverse range of diseases is due to its arsenal of virulence and fitness attributes (117). Attributes such as phenotypic switching between a yeast and hyphal form, expression of adhesins, invasins and hydrolytic enzymes and biofilm formation are considered virulence factors that contribute to its ability to cause disease (62). Additionally, $C$. albicans displays a robust transcriptional response to changes in environment such as fluctuations in $\mathrm{pH}$, nutrient acquisition and stress response which are fitness characteristics that allows this organism to colonize or invade a variety of niches within the human host. 
Phenotypic changes. $C$. albicans has the ability to grow as a yeast cell or in filamentous form either as elongated cells with constrictions as septa (pseudohyphae) or as true hyphae with parallel separations in the cell wall. The ability to transition between yeast and filamentous form is important for its pathogenicity (79). The hyphal form is important for invasion of tissues while the yeast form is primarily responsible for its dissemination. Strains that have been constructed to be unable to transition to filamentous form show attenuated virulence (101). There are several environmental conditions that cue the transition of $C$. albicans to grow in filamentous form. Alkaline conditions $(\mathrm{pH}>$ 7), presence of serum, $\mathrm{N}$-acetyl-glucosamine, physiologic temperature, low cell density $\left(>10^{7}\right.$ cells $\mathrm{ml}^{-1}$ ) and $\mathrm{CO}_{2}$ all promote hyphal growth (117). Hyphal formation is also linked to the expression of a subset of genes associated with virulence such as HWP1, $A L S 3$ and the secreted aspartyl proteases SAP4, SAP5 and SAP6.

C. albicans isolates that have become homozygous at the mating type locus (MTLa/a or $M T L \alpha / \alpha$ ) can also switch between the white yeast form to an elongated opaque form (80). Opaque cells are the mating competent form of this species and can mate with other mating-competent isolates to produce progeny. When compared to white yeast cells, opaque cells exhibit a distinct genetic expression pattern and data suggest they are better colonizers of the skin (and GI tract) but also show attenuated virulence in mouse models (126). Although opaque cells have been reported to be more susceptible to killing by neutrophils than white cells, they also don't release a polymorphonuclear (PMN) chemoattractant, which suggests they avoid detection of neutrophils $(93,94)$. A recent study by Sasse et al. compared internalization of white yeast, filamentous and opaque cells by various phagocytic cells. Their data demonstrate that although opaque cells avoid recognition by human neutrophils on a glass slide, they cannot avoid detection and are efficiently phagocytosed by dendritic cells and by neutrophils in a collagen matrix environment (167).

Adhesins and invasins. In order for C. albicans to become pathogenic, three different processes occur: (i) adherence, (ii) invasion, and (iii) induction of host damage (62). C. albicans is able to adhere to other microbial cells, abiotic surfaces in addition to host cells. Adherence is important in both the commensal and the pathogenic state (33). In $C$. albicans, adherence is mainly regulated by adhesins such as the agglutinin-likesequence $(A L S)$ family, and also by hyphal wall protein 1 (Hwp1) (117). ALS genes encode glycosylphosphatidylinositol (GPI)-linked cell surface glycoproteins. The $A L S$ family is composed of eight members (Als1-7 and Als9) of which Als3 is particularly important. In addition to Als3, Hwp1, which is a hypha-associated GPI-linked protein, is also important for adherence (117).

Invasion of $C$. albicans cells can occur by two different mechanisms: induced endocytosis by host cells and active penetration by C. albicans $(33,212)$. Als3, along with a member of the heat-shock protein 70 (Hsp70) family, Ssa1, are considered invasins and these proteins have been shown to trigger endocytosis by inducing host cytoskeletal arrangments; however, this process does not require viable fungal cells (129, 184). The more predominate form of invasion is active penetration. Hyphae penetrate the 
cell wall by using physical force in combination with hydrolytic enzymes, specifically serine-aspartic-proteases (SAPS). The molecular mechanisms behind active penetration have not all been defined. Although this process results in the host secretion of proinflammatory cytokines and activation of host cellular immunity, hyphae invasion is assumed to play a role in gaining access to deep tissue and the blood stream (117).

Biofilms. C.albicans is the third leading cause of catheter related infections, which serve as reservoirs for pathogens by releasing cells into the blood stream (29). Biofilm formation in C. albicans occurs through a number of different phases including attachment, yeast cell proliferation, hyphal cell formation, extracellular matrix accumulation and dispersion of yeast cells from the mature biofilm $(104,117)$. Transcription factors such as Bcr1, Tec1 and Egf1 are important regulators of biofilm formation and strains constructed to be defective for these regulators in addition to NDT80, ROB1 or BRG1 were deficient in biofilm formation (132). Dispersion of cells from a mature biofilm contributes to its pathogenicity as dispersed cells have been shown to be more virulent in a mouse model (191). Mature biofilms also exhibit higher resistance to antifungal agents and to host immunity than planktonic cells $(46,48)$.

Environmental stress response. C. albicans possesses a number of stress responses that facilitates its survival in a number of challenging and changing niches within the host. Pathways involved in stress response are responsible not only for its survival but are also required for virulence. Phagocytic cells within the host produce highly reactive oxygen species (ROS), reactive nitrogen species (RNS) and antimicrobial peptides that cause oxidative and nitrosative stress to the pathogen (117). C. albicans produces catalase Cta1 and superoxide dismutases Sod1 and Sod5 to detoxify ROS. Likewise, in a response to nitrosative stress, flavohemoglobin-related protein Yhb1 mediates the detoxification of RNS $(77,116,207)$. Host macrophages and neutrophils can effectively phagocytose fungal pathogens and create a nutritionally starved environment (54). Inside these cells, C. albicans switches from glycolysis to gluconeogenesis while lipids and amino acids can serve as nutrient sources. In addition, induction of hyphae while inside the phagocyte can pierce the immune cell and permit escape $(61,106)$.

Stress signals are also transmitted by the mitogen-activated protein (MAP) kinase pathways, which require a series of phosphorylation events. The three MAP kinase pathways in C. albicans are the Mkc1 (MAP kinase from C. albicans), Hog1 (high osmalarity glycerol response) and the Cek1 (Candida ERK-like response) pathways (4, $32,41)$. These pathways facilitate processes such as cellular integrity, biofilm formation, osmotic and thermal stress, filamentation in addition to mating. Strains deficient in these pathways are shown to have decreased virulence (41).

\section{Infections caused by Candida species}

Candida species are the most common cause of invasive fungal infections (IFIs), which include blood stream infections (BSIs) deep tissue infections and also organ disseminated disease (143). Numerous surveys have shown that the frequency of 
candidemia and invasive candidiasis (IC) has increased world-wide in the last three decades, which merits study of infections caused by Candida species. According to a recent survey which describes the epidemiology and outcomes of candidemia in 3,648 patients enrolled in the Prospective Antifungal Therapy (PATH Alliance) registry, 97\% of all infections are caused by five different species of Candida. In order of prevalence, these species are C. albicans (42.1\%), C. glabrata (26.7\%), C. parapsillosis $(15.9 \%), C$. tropicalis (8.7\%) and C. krusei (3.4\%) (143). This Candida species hierarchy varies in different areas of the world $(99,204)$. Among the medically relevant fungal species, $C$. albicans is the most frequently recovered from human samples in the United States. This organism is responsible for a wide range of infections, from infection of superficial mucosal surfaces to invasive life-threatening disseminated disease. Invasive fungal infections are associated with health care costs over $\$ 32,000$ per patient combined with an increase of about seven days of hospital stay (9).

Candida species are part of the normal flora that exist commensally in over $70 \%$ of the healthy population (58). Individuals of advanced age or have oral prosthesis, such as dentures, exhibit higher instances of colonization. Colonization is defined as Candida species generated from one or more samples from non-sterile sites and the extent of colonization has been repeatedly linked to subsequent infection $(3,97,150,196)$. Previous work has shown that isolates responsible for invasive disease are often of the same karyotype of those of which the patient is colonized (134). Patients treated extensively with broad spectrum antibiotics can lead to an over-colonization of Candida species and may be at higher risk for IC. Other risk factors include gastrointestinal surgery, chemotherapy, immunosuppression, or other invasive medical treatments. Mortality associated with IC has been estimated between 14.5\% - 49\% (139).

Mucocutaneous candidiasis can be classified into genitourinary and non-genitourinary candidiasis. The most common type of genitourinary candidiasis is vulvocandidiasis (VVC), which usually occurs in healthy women. It is estimated that $75 \%$ of women are diagnosed with VVC at least once in their childbearing years and although still less common than bacterial vaginosis, VVC is the second most common diagnosis (40\%) in woman with vaginal complaints in the primary care center $(2,7)$. Although Candida species may asymptomatically colonize the vaginal cavity, $\mathrm{VVC}$ is associated with clinical signs of inflammation. In studies where Candida species where identified in VVC infections, C. albicans was the most commonly isolated causative organism followed by C. glabrata (2). Recurrent vulvocandidiasis (RVVC) is defined as over four VVC diagnosis within one year and in these cases, long term antifungal suppressive therapy is indicated. Of note, incidence of the non-albicans species, specifically $C$. glabrata, are more prevalent in RCCV than VVC. Non-albicans species are also more prevalent in women with HIV, who have uncontrolled diabetes and who are advanced in age $(35,181,195)$.

The diagnosis and management of candiduria remains controversial among providers. Candiduria is prevalent among critically ill patients and studies show a positive correlation between candiduria and mortality, however, the role of candiduria as an indicator of invasive disease is unclear (196). The differential diagnosis between a 
urinary tract infection (UTI), colonization and sample contamination is difficult so the decision to treat is often provider specific (49). The most common risk factor for candiduria is colonization of urinary catheters, which may mask the signs and symptoms of infection. Alternatively, hematogenous spread is the most common etiology of renal candidiasis; a much more serious diagnosis is an indication to treat with aggressive antifungal therapy (71).

Oral pharyngeal candidiasis (OPC) is the most common opportunistic infection with Human Immunodeficiency Virus (HIV) (140). OPC is more common in patients with low $\mathrm{CD} 4^{+}$count or high HIV titers however, since the widespread implementation of highly active antiretroviral therapy (HAART), OPC rates have declined $(37,42)$. Even in the era of HAART therapy and fluconazole prophylaxis, $C$. albicans is still the most commonly detected species (62\%), followed by C. glabrata (17\%) and C. dubliniensis (12\%) (140). This non-albicans shift in the causative etiology of OPC creates concern regarding azole resistance.

\section{Treatment of Candida infections}

There are currently only four structurally distinct antifungal classes available for the treatment of invasive candidiasis. Unlike bacteria, both fungal cells and mammalian cells are eukaryotic, which makes targeting fungal-specific processes more difficult. The most exploited strategy for antifungal development targets the ergosterol biosynthesis pathway or ergosterol incorporated into the fungal cell membrane. Other strategies include inhibiting RNA and DNA synthesis by compounds that are selectively transported into the fungal cell and inhibition of the fungal cell wall. Mortality rates in neutropenic patients for yeast and mold infections are still unacceptably high and time to implementation of the correct antifungal has a significant impact on patient outcomes (57).

Amphotericin B. Amphotericin B (AmB) is a polyene macrolide antifungal that was first isolated from Streptomyces nodosus in the 1950s. Over the past five decades, amphotericin B deoxycholate formulation (AMBd; Fungizone) has been the goldstandard for the treatment of life-threatening fungal infections and generally remains refractory to resistance. Its primary fungicidal mechanism of action is direct binding to ergosterol; the primary sterol found in the fungal cell membranes. Its dual complementary action of aggregating to form membrane-permeabilizing ion channels increases its potency but is not required for fungicidal activity (63). Pharmacodynamic studies demonstrate that fungicidal activity can be enhanced by optimizing the concentration-to-MIC ratio (89). Unfortunately, the clinical utility of AMBd is often times limited by its associated adverse effects such as nephrotoxicity and infusion-related reactions (39). Because of these treatment-limiting adverse effects, newer lipid-associated formulations of amphotericin B (Ambisome, Abelcet and Amphotec) have replaced conventional AMBd as first-line therapy for many types of infections. 
Flucytosine. $(5 \mathrm{FC})$ is a pyrimidine analog that inhibits DNA replication and protein synthesis. Flucytosine is selectively transported into the fungal cell by cytosine permease and is then deaminated by cytosine deaminase to 5-fluorouracil. It is then converted to its active form, 5-fluorodeoxyuridylic acid to inhibit DNA synthesis (194). Due to the rapid development of resistance, $5 \mathrm{FC}$ is rarely used as monotherapy. In a murine model of disseminated candidiasis, time over MIC was the best pharmacodynamic parameter correlated with efficacy (8). Although the Clinical Practice Guidelines for the Management of Candidiasis recommend 5FC in combination with amphotericin B or fluconazole for a variety of infection types, concerns regarding toxicity such as bone marrow suppression and hepatic transaminase elevation often times prevent its use (139). Its current utility is primarily limited to treatment of cryptococcal meningitis (159).

Echinocandins. Echinocandins are the newest members in the spectrum of antifungals. Current approved agents by the U.S. Food and Drug Administration are caspofungin (Cancidas; Merck and Co.), micafungin (Mycamine; Astellas Pharmaceuticals) and anidulafungin (Eraxis; Pfizer Pharmaceuticals). Echinocandins are used for treatment of invasive candidiasis based on results of three defining clinical trials in which caspofungin, micafungin and anidulafungin were shown non-inferior to amphotericin B, liposomal amphotericin B and fluconazole, respectively $(92,121,154)$. These agents are characterized by their broad spectrum of activity, low incidence of drugdrug interactions and a relative lack of adverse effects (211). Echinocandins are lipopeptides that bind to $1,3-\beta$-D glucan synthase, inhibiting production of $\beta-1,3$ glucan which, along with chitin, is responsible for integrity and shape of the fungal cell wall. $1,3-\beta$-D glucan synthase is a multi-unit enzyme that is made of a catalytic subunit Fksp, the target of echinocandins, and the regulatory unit Rholp. All three echinocandin agents are fungicidal in a dose dependent fashion against the Candida species including those isolates resistant to azole antifungals $(137,146,147,180)$. Unlike the azole class, echinocandins are noted to retain activity against fungal biofilm and biofilm formation (180).

Azole antifungals. Members of the azole class are the most widely used of all antifungals. Their utility is mainly due to their activity against a broad spectrum of fungal pathogens and a mild spectrum of side effects, specifically lacking the treatment limiting nephrotoxicity experienced by patients undergoing treatment with AmB. There are four azoles currently used for the treatment of invasive fungal infections. Although these agents are all of the same class, they vary individually in regards to spectrum of activity, pharmacokinetic and toxicity profiles. Appropriate utilization of these agents is based upon these unique characteristics.

The primary target of all azole antifungals is 14- $\alpha$ lanosterol-demethylase, which is the product of the $E R G 11$ gene (209). Lanosterol demethylase is a member of the cytochrome P450 (CYP450) class of enzymes, and is a critical enzyme in the biosynthesis of ergosterol. As ergosterol is not found in the mammalian cell membrane, inhibition of this pathway is a fungal specific mechanism of action for this class. Treatment with azoles results in decreased cellular ergosterol and leads to the 
accumulation of toxic methylated sterols. Azoles are considered fungistatic for Candida and other yeast species but some are fungicidal to Aspergillus species (110).

Fluconazole (FCZ; Diflucan; Pfizer). Fluconazole is available in both an oral and intravenous and has been widely studied in treating invasive fungal disease. When compared to $\mathrm{AmB}$, fluconazole is defined by a limited toxicity profile, but its spectrum of activity is limited to yeast. Species such as $C$. krusei display inherent resistance to this agent and $C$. glabrata has been observed to exhibit both inherent and acquired resistance secondary to treatment $(136,144)$. Fluconazole has excellent bioavailability independent of $\mathrm{pH}$ or concomitant meals and exhibits linear kinetics $(65,214,215)$. This agent also exhibits limited protein binding $(\sim 10 \%)$, a high volume of distribution and achieves appreciable concentrations in the cerebrospinal fluid $(\mathrm{CSF})(16,187)$. Fluconazole is primarily eliminated unchanged in the urine and requires dosage adjustment in renal insufficiency (15). As fluconazole is a moderate inhibitor of CYP450 enymes 3A4, CYP2C9 and CYP2C19, it potentially interacts with the metabolism of a wide variety of drugs (131). In addition, fluconazole also inhibits uridine diphosphate (UDP)glucuronosyltransferase (UGTs), which mediates drug metabolism by glucuronidation (190). Like all triazole antifungals, area under the concentrations curve (AUC) to MIC is the best pharmacodynamic parameter that predicts azole antifungal activit (89).

Itraconazole (ICZ; Sporanox; Janssen Pharmaceuticals). Itraconazole, voriconazole, and posaconazole are all considered extended-spectrum triazoles as they exhibit greater potency than fluconazole against most fungal species. Itraconazole is effective against most yeast however, $C$. krusei and C. glabrata may show cross resistance with FCZ (144). When compared to fluconazole, itraconazole gains activity against certain molds such as Aspergillus and dimorphic endemic fungi. Itraconazole is only available orally and is formulated as either a capsule or a solution. Oral bioavailability of itraconazole is significantly less than fluconazole (213). The oral solution exhibits better bioavailability than the capsule, and this improves under acidic fasting conditions (214). Itraconazole is highly protein bound and is metabolized in the liver by CYP3A4, which results in the formation of the pharmacologically active metabolite hydroxyitraconazole $(151,186)$. Itraconazole is a strong inhibitor of CYP3A4 and has the potential for numerous drug-drug interactions (78).

Voriconaozle (VCZ; Vfend; Pfizer). Voriconazole is also an extended spectrum triazole and is structurally related to fluconazole. Voriconazole displays excellent activity against Candida spp. including C. krusei and is the drug of choice for treatment of invasive aspergillosis (68). Voriconazole exhibits activity against endemic fungi but notably lacks activity against Zygomycetes. Voriconazole is formulated in orally and also intravenously with a cyclodextrin carrier. Like fluconazole, voriconazole is well absorbed and displays high bioavailability upon oral administration (81). Voriconazole displays non-linear kinetics and absorption is independent of $\mathrm{pH}$ and food administration. Voriconazole is moderately protein bound ( $58 \%)$ and demonstrates CSF penetration (107). Unlike itraconazole, voriconazole is primarily metabolized by CYP2C19. The argument has been made for therapeutic drug monitoring (TDM) of voriconazole levels due to inter-patient genetic differences in CYP2C19 activity $(40,202)$. Renal function 
should be monitored in patients receiving the intravenous formulation due to the sulphobutylether-beta-cyclodextrin carrier (138).

Posaconazole (PCZ; Noxafil; Schering Corporation). Posaconazole is the newest addition to the triazole class and is structurally related to itraconazole. Global surveillance studies have noted that posaconazole is more active than itraconazole and fluconazole, and as effective as voriconazole against a diverse population of clinically relevant Candida spp $(148,149)$. Posaconazole is available as a $40 \mathrm{mg} / \mathrm{mL}$ oral suspension and due to its lipophilicity, should be administered with a full meal or a nutritional supplement (27). Absorption of posaconazole is saturable so the administration of PCZ in divided doses has been shown to increase the relative bioavailability when compared to a single dose (45). Additionally, gastric $\mathrm{pH}$ also plays a role in posaconazole absorption so administration with an acidic beverage is recommended while coadministration with acid suppressing drugs may decrease absorption (25). Posaconazole is highly protein bound and distributes widely to the tissues. Metabolism is largely mediated by UGT (glucoronosyl transferase) which allelic polymorphisms have been shown to alter pharmacokinetics (60). Excretion of posaconazole has also been shown to be mediated in part by P-glycoprotien and inducers of P-glycoprotein have been shown to increase clearance (26). Posaconazole is not metabolized significantly by CYP450 enzymes but remains an inhibitor of CYP isoenzyme $3 \mathrm{~A} 4$ which can contribute to drug interactions (91).

\section{AZOLE RESISTANCE IN CANDIDA ALBICANS}

\section{Clinical impact of azole resistance}

Candida spp are the principle causative organisms of orophyryngeal (OPC) candidiasis and also the fourth most common pathogen isolated from nosocomial bloodstream infections. Due to the implementation of Highly Active Antiretroviral Therapy (HAART) and fluconazole prophylaxis, the incidence of OPC among $\mathrm{HIV}^{+}$ patients has declined (42). There is a population that for various reasons is noncompliant with their drug regimen, do not have access to antiretrovirals or do not tolerate HAART therapy for whom OPC remains a problem. Recent studies and current IDSA treatment guidelines show that for AIDS patients, recurrent prophylactic fluconazole regimens for OPC results in decreased colonization and did not appear to cause resistance $(139,140)$. Data show that the likelihood of resistant yeast was increased in patients with recurrent candida esophagitis and denture use (140). In these cases, the prolonged and repeated fluconazole treatment course has created a fluconazole-resistant population of $C$.

albicans. Repeated fluconazole treatment has created a non-albicans shift in the causative organisms of OPC, many of which have decreased susceptibilities to azoles (188).

The increased incidence of invasive candidiasis is largely a result of progress in medical technology or therapies that have created an immunosuppressed population susceptible to fungal pathogens. Invasive candidiasis has a significant impact on patient 
outcomes and medical costs. The mortality attributable to invasive disease varies by source. The IDSA guidelines state that mortality attributable to disseminated candidiasis ranges between $14.5-49 \%$ in adults and $10-15 \%$ in neonates and children (139). As patients who acquire invasive candidiasis often have multiple comorbidities, the mortality attributed to candidiasis is difficult to calculate. As fluconazole has been the "work horse" of antifungal therapy, a non-albicans shift has also been observed in nosocomial isolates that may be less sensitive to azole treatment.

Resistance in Candida species is often distinguished into pathogens who exhibit intrinsic versus acquired resistance. Species such as $C$. krusei and some of the $C$. glabrata population exhibit intrinsic resistance, meaning resistance without prior introduction to the drug. C. glabrata and other Candida species can acquire resistance in response to a drug challenge. Recently, the Clinical Laboratory and Standards Institute (CLSI) have redefined their standards for in vitro antifungal susceptibility testing. Current standards now consider species-specific clinical break points (CBP) using established epidemiological cutoff values (ECV), defined as the upper cutoff value for wild-type MIC, pharmacokinetic (PK)/pharmacodynamic (PD) parameters and relationship between MIC and clinical outcome. Although previous CLSI CBPs reasonably predicted therapeutic success or failure, the new CBPs exhibit increased sensitivity in the detection of emerging resistance (145). The current interpretation of in vitro susceptibility testing of $C$. albicans after 24-hour incubation to fluconazole is "susceptible" $\leq 2 \mu \mathrm{g} / \mathrm{mL}$, "susceptible dose dependent" $4 \mu \mathrm{g} / \mathrm{mL}$ and "resistant" isolates have an MIC of $8 \mu \mathrm{g} / \mathrm{mL}$. Break points vary regarding species and antifungal.

\section{Molecular mechanisms of azole resistance in $C$. albicans}

The efficacy of fluconazole is decreased in clinical isolates of C. albicans by the interplay of several mechanisms of resistance. The azole antifungal agents inhibit the biosynthesis of ergosterol, the major membrane sterol of fungi. By binding to the heme in the active site of the cytochromeP450 enzyme, lanosterol demethylase, the azoles inhibit its activity (59). We currently do not know the mechanism of azole uptake into the fungal cell but recent kinetic data supports the hypothesis that its uptake is facilitated (113). Several mechanisms of resistance to the azole antifungal agents directly involving ERG11 have been described in C. albicans. One major mechanism involves accumulation of point mutations in the gene encoding lanosterol demethylase (ERG11) (162). These mutations have long been accepted to confer azole resistance by producing conformational changes that prevent effective binding between the azoles and their target while not affecting the function of this enzyme in ergosterol biosynthesis $(5,15)$. In addition to mutations in $E R G 11$, constitutive overexpression of this gene requires higher concentrations of intracellular fluconazole for sufficient inhibition. ERG11 overexpression occurs by either gain-of-function mutations in the transcriptional regulator $U P C 2$ or by instances of aneuoploidy, specifically, increased chromosome 5 copy number, on which ERG11 resides (discussed below). 
A less common mechanism of azole resistance is alterations in the ergosterol biosynthesis pathway which results in alternate sterols in the fungal cell membrane. This usually occurs by inactivation of sterol $\Delta^{5,6}$-desaturase, encoded by $E R G 3$ (125). Erg3 converts nontoxic $14 \alpha$-methylated sterol intermediates that accumulate due to azolemediated Erg11 inhibition, into the toxic sterol 14 $\alpha$-methylergosta-8,24(28)-dien-3 $\beta, 6 \alpha-$ diol (193). Non-functional $E R G 3$ alleles result in high-level azole resistance by allowing cells to avoid the synthesis of the toxic sterols. Inactivation of this enzyme has been shown to result from either a nonsense mutation in $E R G 3$ or a SNP, which allegedly inactivates the enzyme (119). Although strains containing these inactivating mutations are usually unable to filament and display decreased virulence, isolates containing inactive Erg3 yet are still filamentous and do not display decreased virulence have been documented $(123,193)$.

Overexpression of efflux pumps is a common mechanism of resistance to drugs toxic to the fungal cell. In C. albicans, there are two families of efflux transporters which are important in azole resistance $(23,43,125)$. Two ATP Binding Cassette (ABC) transporters, $C D R 1$ and $C D R 2$, harness the energy from ATP hydrolysis to actively transport fluconazole from the cell while the Major Facilitator Superfamily (MFS) transporter, $M D R 1$, requires a proton gradient to drive efflux. The CaCDRl gene is a homologue to the PDR5 ABC efflux transporter in $S$. cerevisiae. $C a C D R 1$ was shown to functionally compliment a $S$. cerevisiae $p d r 5 \Delta$ mutant by restoring fluconazole susceptibility phenotypes (152). Disruption of CDR1 in C. albicans resulted in increased levels of intracellular fluconazole and increased sensitivity not only to azoles but also other drugs (164). CDR2 is very similar to $C D R 1$ but disruption of this pump alone does not affect fluconazole susceptibilities (164). The disruption of $C D R 2$ in a mutant already deficient for $C D R 1$ further increases susceptibility to fluconazole (164). Similar studies were done for $C a M D R 1$ however, overexpression of this transporter increased resistance specifically to fluconazole and not other azoles. In a fluconazole-susceptible isolate, these efflux pumps are usually expressed in a low or non-detectable level but expression is stimulated in response to azoles and other chemicals (25). In fluconazole-resistant strains, constitutive overexpression of the $C D R 1, C D R 2$ and $M D R 1$ genes is commonly observed. Our lab and others have shown constitutive overexpression of $C D R 1, C D R 2$ and MDR 1 is due to activating mutations in zinc-cluster transcriptional regulators, Tac1 and Mrr1 respectively $(3,5)$. Analysis of mutants where genes encoding for these transporters are disrupted has proven that the contribution of $C D R 1$ is greater than $C D R 2$ to multidrug resistance (189). In addition, post-transcriptional studies have demonstrated that azoleresistant isolates do not only display higher transcription initiation rates, but CDR 1 mRNA is more stable when compared to the azole-susceptible counterpart (112).

The genome of $C$. albicans can tolerate changes in chromosomal rearrangement and chromosomal aneuploidies (124). Although reproduction is primarily asexual, a parasexual cycle has been identified in this species. C. albicans does not undergo meiosis therefore, the tetraploid result of mating undergoes recombination mediated by the endonuclease Spo11 and concerted chromosomal loss by mitosis, which results in a high degree of aneuploidy (51). C. albicans has also been observed to undergo substantial genetic variation in a short time in a murine model of infection (52). A study that 
observed the chromosomal abnormalities that result from fluconazole exposure demonstrated the rapid acquisition of isochromosome 5L [i(5L)] in response to drug (174). Both ERG11 and TACl reside on chromosome 5 and increased chromosome 5 copy number has been shown to contribute to azole resistance in a clinical isolate (173). Genetic abnormalities have been detected on all eight chromosomes in C. albicans but these changes are usually transient and will normalize without drug pressure. Other conditions promote genetic abnormalities such as low nitrogen containing media in addition to growth on specific carbon sources such as l-sorbose or d-arabinose (124). These established mechanisms of resistance are all able to individually contribute to azole resistance but high-level resistance is mainly due to multiple mechanisms of resistance that accumulate over time (155).

\section{TRANSCRIPTIONAL REGULATORS OF AZOLE RESISTANCE}

\section{Zinc-cluster transcription factors}

Zinc-cluster transcription factors (TFs) are a class of exclusively fungal zinc-finger proteins comprised of six cysteine residues bound to two zinc atoms $\left(\mathrm{Zn}(\mathrm{II})_{2} \mathrm{Cys}_{6}\right)(109)$. Zinc-cluster transcription factors recognize the conserved DNA motif $\mathrm{CX}_{2} \mathrm{CX}_{6} \mathrm{CX}_{5-}$ ${ }_{12} \mathrm{CX}_{2} \mathrm{CX}_{6-8} \mathrm{C}$ and bind as either monomers, homodimers or heterodimers. Apart from the cysteine rich DNA binding domain (DBD), zinc-cluster TFs also contain regulatory and activation domains. Included in their target gene repertoire, many zinc-cluster TFs regulate the expression of other zinc-cluster proteins or may also auto-regulate.

The best studied zinc-cluster family member is Gal4. Gal4 regulates genes involved in the transport and metabolism of galactose via upstream activation sequences $\left(\mathrm{UAS}_{\mathrm{GAL}}\right)$ in their promoters (102). Gal4 is localized in the cytoplasm and upon activation, is directed towards and imported into the nucleus facilitated by a characterized nuclear localization signal (NLS) (20). In the absence of galactose, Gal4 is inhibited by the repressor protein Gal80 (206). Relief of inhibition by Gal80 is mediated by its interaction with Gal3, a relationship which is not completely understood, but is dependent upon the presence of galactose $(13,141)$. Although not necessary for activation, Gal4 undergoes a series of phosphorylation events on serine residues 691,696 and 837. These modifications are thought to help "fine tune" Gal4 activation (160). Gal4p turnover may also be post-transcriptionally regulated by the ubiquitin degradation system (128). Other zinc-cluster proteins are constitutively localized in the nucleus bound to their target promoters. Large scale and targeted protein localization studies have increased our insight of cellular location of zinc-cluster transcription factors in budding yeast $(75,115)$.

As DNA in chromosomes are wound tightly and organized into chromatin, zinccluster proteins require the participation of chromatin remodeling machinery, histonemodifying enzymes and co-activators to activate genes. Transcription of several genes in $S$. cerevisiae are known to be dependent on histone actetyltransferases such as Spt-AdaGcn5-acetyltransferase, known as the SAGA complex. Previous work has shown that 
ScPdr1, a zinc-cluster TF and the primary regulator of the $\mathrm{ABC}$ transporter PDR5, interacts with the SAGA complex to activate transcription (55). Likewise, Ume6, a repressor zinc-cluster TF, relies on a histone deacetylase complex (HDAC) to exert its regulatory control $(83)$.

\section{Zinc-cluster transcription factors and azole resistance in $C$. albicans}

Constitutive activation of efflux pumps $M D R 1, C D R 1$ and $C D R 2$ are a prevalent and important mechanism of multidrug resistance in $C$. albicans. Studies performed in $S$. cerevisiae demonstrate that $C a C D R 1$ and $C a C D R 2$ can functionally compliment a strain the lacks ABC transporter PDR5 $(152,163)$. Regulatory elements for both genes are defined.(36) The promoter region of $C D R$ l contains both a basal expression element (BEE), responsible for basal expression, and a drug responsive element (DRE), which is required for fluphenazine and estradiol induction of expression. Only a single DRE was detected in the promoter of $C D R 2$ but it shared a 21 base pair consensus sequence with CDR1. This consensus DRE (5'-CGGA(A/T)ATCGGATATTTTTTTT-3') contains two CGG triplet repeats suggesting the involvement of a zinc-cluster transcription factor in their regulation. In 2004, Coste et al, established the role of zinc-cluster transcription factor, $T A C 1$ (transcriptional activator of $C D R$ genes) as the regulator of both $C D R 1$ and $C D R 2$ overexpression in response to fluphenazine (23). Tac1 is localized to the nucleus and GOF mutations in azole resistant isolates are prevalent, resulting in the constitutive overexpression of drug efflux transporters (22). Hyperactive TAC1 alleles have been shown to be co-dominant with wild-type alleles and resistant phenotypes occur when strains are homozygous for a GOF TAC1 allele (22). The mechanism by which homozygous $T A C 1$ alleles arise is by recombination between portions of chromosome 5 or by duplication of a portion of chromosome 5, on which TAC1 resides. Tac1 also indirectly induces the transcription of $P D R 16$ encoding a phosphatidylinositol transfer protein (216). As CDR1 and CDR2 function as general phospholipid translocators in addition to their role in pleiotropic drug resistance, this link demonstrates the importance of these two processes for C. albicans survival in a wide range of environments (216). A recent study has demonstrated that artificial activation of zinc-cluster transcription factor designated Mrr2 can also increase $C D R 1$ transcription with similar efficacy to Tac1 (168). Unlike Tac1, Mrr2 was selective for $C D R 1$ expression and not $C D R 2$. The clinical significance of Mrr2 in azole resistance is not yet known.

$M D R 1$ constitutive expression in C. albicans is also a frequent cause of azole resistance in clinical isolates. The mechanism of $M D R l$ overexpression in azole resistance was identified using genome-wide profiling analysis of match isolate pairs of fluconazole-susceptible and fluconazole-resistant counterparts that overexpressed MDR1 (127). Orf19.7372, designated $M R R 1$ was overexpressed in all three resistant isolates and disruption of MRR 1 in a fluconazole susceptible background abolished MDR1 expression in response to fluconazole. Sequence analysis of resistance isolates revealed GOF mutations in $M R R 1$. As seen with $T A C 1$, the majority of $M D R 1$ overexpressing strains had become homozygous for the $M R R l$ GOF allele either through recombination between chromosome 3 homologous, on which $M R R 1$ resides, or may also occur by 
chromosomal duplication events, which results in two mutated MRRI alleles combined with a complete loss of the wild-type allele (43). An artificially activated form of the transcription factor Cap1 also results in increased $M D R 1$ expression and to a lesser degree, Upc2 containing a GOF mutation can increase MDR 1 expression in a MRR1 dependent fashion (120). Adding another level of transcriptional regulation is the involvement of the MADS box transcription factor Mcm1, which is essential for MDR1 overexpression by activated Mrr1 but unnecessary for activated Cap1 (120).

In clinical-azole resistant isolates of $C$. albicans, constitutive overexpression of ERG11 and other genes in the ergosterol biosynthesis pathway (ERG genes) is observed. This is often a result of transcriptional activation of Upc2 (177). Previous work has identified Upc2-specific sterol response elements (SRE) core motif in the promoter region of ERG11 and other ERG genes (135). Deletion of UPC2 in a fluconazolesusceptible background results in hypersusceptibility to fluconazole, diminished ergosterol levels in the cell membrane and the inability to increase ERG11 expression in response to drug treatment $(135,177)$. Introducing a triple hemagglutinin epitope tag at the $\mathrm{C}$ terminus of $U P C 2$, the putative activation domain, resulted in a gain-of-function effect in the fusion protein, resulting in increased expression of Upc2 target genes (108). CHIP-Chip analysis has also identified Upc2 to be enriched in the promoters of genes involved in ergosterol biosynthesis and other pathways. In a fluconazole-resistant clinical isolate, our lab first identified a GOF mutation in UPC2 resulting in constitutive overexpression of genes involved in the ergosterol biosynthesis pathway in addition to genes involved in the oxidative stress pathway (44). Our recent data show that a variety of UPC2 GOF mutations are prevalent in azole-resistant clinical isolates and these mutations contribute to azole resistance (50). Notably, our lab and others have observed clinical isolates that overexpress ERG11 yet do not contain UPC2 GOF mutations, which suggest alternative mechanisms of ERG11 overexpression.

FCR 1 has been previously characterized as a zinc-cluster transcription factor and its contribution to pleiotropic drug resistance has been studied in $S$. cerevisiae. Talibi and Raymond expressed C. albicans FCR1, FCR2 and FCR3 genes in S. cerevisiae and showed that that these genes were able to increase the expression of PDR5, which encodes a transporter in the ATP binding cassette (ABC) superfamily and participates in the efflux of azole antifungals (185). Surprisingly, $f c r l \Delta / f c r l \Delta$ mutant strains were more resistant to fluconazole, ketoconazole, itraconazole, and Brefeldin A. Therefore, characterization of $F C R 1$ as either a positive or negative regulator of antifungal resistance as yet to be clarified in $S$. cerevisiae. In addition to the FCR 1 pathway, it has been shown that azole resistant isolates ability to grow in the presence of fluconazole is abrogated in the presence of a calcineurin inhibitor (17). Targets of calcineurin include the genes $C R Z 1$ and $C R Z 2$, which are also transcription factors and have been linked to fluconazole susceptibility. In a study by Bruno and Mitchell, mutants with the crzl homozygous null genotype, like when treated with cyclosporine A, would reverse the ability of azole resistant mutants to grow in the presence of drug (17). 


\section{CENTRAL HYPOTHESIS AND RESEARCH OBJECTIVES}

Candida albicans is an opportunistic fungal pathogen that causes mucosal, cutaneous, and systemic infections, including oropharyngeal candidiasis (OPC), the most frequent infection in people with AIDS. Fluconazole and other azole antifungal agents have proven effective in the management of OPC; however, with increased use of these agents, treatment failures have occurred that have been associated with the emergence of azoleresistant strains of $C$. albicans. The long term goal is to improve the treatment of Candida infections by understanding the molecular basis of azole resistance. The overall objective of this dissertation was to understand mechanisms by which Erg11 mediates resistance to fluconazole. Three specific aims were developed to investigate this overall objective.

In a large group of clinical C. albicans isolates enriched for azole resistance, I created a transcriptional profile defining expression of genes known to cause azole resistance such as ERG11,CDR1,CDR2 and MDR1. ERG11 was found to be upregulated in almost three-fourths of the fluconazole-resistant isolates examined. These data suggest that ERG11 overexpression is a common contributor to fluconazole resistance in C. albicans. Prior to this work, only 3 GOF mutations in Upc2 (G648D, A643T and A643V) have been reported in characterized genetically-matched sets of isolates. In light of this preliminary evidence, I hypothesized GOF mutations in UPC2 were responsible for the majority of ERG11 overexpression in this collection. My first specific aim was to investigate the prevalence and role of $U P C 2$ gain-of-function mutations in the overexpression of ERG11 in fluconazole-resistant C. albicans isolates. Among the ERG11overexpressing isolates studied, 29 isolates contained at least one amino acid substitution in Upc2. I frequently recovered and characterized eight distinct single-nucleotide substitutions in UPC2. Five of these substitutions in UPC2 have not been described previously. Of the five novel mutations, four mutations resulted in increased ERG11 expression. I determined that transcriptional activation of $E R G 11$ by a variety of GOF mutations in transcription factor Upc2 are prevalent in a large group of azole resistant isolates.

Although GOF mutations in Upc2 were prevalent in this collection, there were several ERG11-overexpressing isolates that did not contain UPC2 mutations. Other groups have also observed ERG11-overexpression in clinical isolates that do not contain GOF mutations in UPC2. These data suggest that there are UPC2-independent mechanisms of ERG11-overexpression. In C. albicans, the Pho-G transcription factor NDT80 has been implicated in azole resistance not only due to its regulation of CDRl but also due to its regulation of genes involved in the ergosterol biosynthesis pathway. Investigation of CaNDT80 has identified putative binding sites in the UPC2 promoter area and ChIP-chip data has shown enrichment of Ndt80 in the promoter region of genes involved in ergosterol biosynthesis. In light of this preliminary data, I hypothesized that GOF mutations in NDT80 result in increased ERG11 expression and azole resistance in clinical isolates. My second specific aim was to investigate NDT80 mutations derived from the resistant component of two genetically matched isolate sets on ERG11 expression and fluconazole resistance. Sequencing of the NDT80 allele of both matched sets revealed several mutations that resulted in amino acid substitutions when compared 
to SC5314. This analysis also showed that a loss of heterozygostiy event occured so that the resistant counterpart was homozygous for one allele. A strain carrying the NDT80 allele derived from fluconazole-resistant isolate 1619 did not result in increased ERG11 expression and increased fluconazole resistance. The mechanism by which ERG11 is upregulated in the absence of UPC2 gain-of-function mutations is currently under investigation.

In addition to transcriptional regulation of the ERG11 gene, it is well known that fluconazole resistance in clinical isolates of C.albicans are associated with specific point mutations in ERG11 but there are no studies that show the effects of these mutations within the pathogen itself. In my third aim, I investigated the contribution of specific amino acid substitutions to a panel of azole antifungals. To investigate this, I sequenced both ERG11 alleles in the 63 unmatched clinical isolates with reduced susceptibility to fluconazole. Sequencing data revealed that 55 of the 63 isolated contained missense mutations in ERG11 that resulted in at least one amino acid substitution. I selected a group of mutant ERG11 alleles and expressed them in SC5314. In this analysis, I was particularly interested in characterizing amino acid substitutions that occurred alone and also when accompanied by another mutation. In total, I characterized ten ERG11 alleles homozygously carrying one amino acid substitution and nine alleles which homozygously carried ERG11 alleles with a combination of amino acid substitutions. Fluconazole, itraconazole and voriconazole susceptibilities were performed for strains carrying each mutant ERG11 allele homozygously with an identical ERG11 mutation. Our data demonstrate many of these mutations resulted in fluconazole resistance, but most single mutations are not as significant when tested against voriconazole or itraconazole. Itraconazole, in particular seemed less effected by ERG11 mutations which produced significant resistance to fluconazole although amino acid combination Y132F and F145L resulted in increased itraconazole resistance. Specific combinations of ERG11 mutations resulted in increased azole resistance beyond single mutations. These data suggest that structural differences between azole effect activity against specific mutant ERG11 alleles. 


\section{CHAPTER 2. GAIN-OF-FUNCTION MUTATIONS IN UPC2 ARE A FREQUENT CAUSE OF ERG11 UPREGULATION IN AZOLE-RESISTANT CLINICAL ISOLATES OF CANDIDA ALBICANS*}

\section{INTRODUCTION}

Candida albicans is an opportunistic fungal pathogen that causes mucosal, cutaneous, and systemic infections, including oropharyngeal candidiasis (OPC), the most frequent infection in people with AIDS $(47,88)$. In the United States, Candida is the fourth-mostcommon organism isolated from nosocomial bloodstream infections and is associated with a mortality rate approaching 40\% (153). Fluconazole and other azole antifungal agents have proven effective in the management of OPC; however, with increased use of these agents, treatment failures have occurred that have been associated with the emergence of azole-resistant strains of $C$. albicans (157). The azole class of antifungals works by inhibiting the cytochrome P450 enzyme lanosterol demethylase, a critical enzyme in the synthesis of ergosterol which is encoded by the ERG11 gene (90). The efficacy of fluconazole is decreased in clinical isolates of $C$. albicans by the interplay of several mechanisms of resistance $(105,125,142,203)$. Overexpression of the efflux transporter genes $C D R 1, C D R 2$, and $M D R 1$ is a common mechanism of drug resistance in this organism $(53,105,165)$. Point mutations in the $E R G 11$ gene result in reduced binding affinity of azoles to their target without precluding enzymatic function (200). In addition to point mutations, overexpression of ERG11 has also been shown to decrease fluconazole susceptibility. ERG11 gene amplification by chromosome 5 duplication or the presence of a chr5L isochromosome is known to contribute to azole resistance (173). The zinc-cluster transcription factor Upc2 has been shown to regulate the expression of ERG11 and other genes involved in ergosterol biosynthesis $(108,177)$. Previous studies show that activating mutations in Upc 2 result in increased expression of ERG11 and decreased fluconazole susceptibility; however, only three substitutions (G648D, A643T, and A643V) in Upc2 have been identified in clinical isolates that contribute to azole resistance $(44,66,74)$. In all cases, these substitutions have been identified in fluconazole-resistant clinical isolates of genetically matched isolate pairs.

In this study, I examined the prevalence of overexpression of $E R G 11$, as well as the overexpression of efflux pump genes $C D R 1, C D R 2$, and $M D R 1$, in a group of 63 unrelated fluconazole-resistant clinical isolates. I also investigated the prevalence of UPC2 mutations among isolates that overexpress ERG11. I determined which UPC2 mutations, both previously described and novel, result in increased ERG11 expression, altered cellular ergosterol content, and increased resistance to azoles, as well as to

*Reprinted with permission of the publisher. Flowers, S. A., K. S. Barker, E. L. Berkow, G. Toner, S. G. Chadwick, S. E. Gygax, J. Morschhauser, and P. D. Rogers. 2012. Gainof-function mutations in UPC2 are a frequent cause of ERG11 upregulation in azoleresistant clinical isolates of Candida albicans. Eukaryot Cell 11:1289-99.. 
terbinafine. Finally, I identified genes that are coordinately differentially expressed in four strains expressing unique $U P C 2$ gain-of-function mutations.

\section{MATERIALS AND METHODS}

\section{Strains and growth conditions}

All C. albicans strains (Table 2.1) were stored as frozen stock in 20\% glycerol at $80^{\circ} \mathrm{C}$ and cultured on YPD (1\% yeast extract, $2 \%$ peptone, and $1 \%$ dextrose) agar plates at $30^{\circ} \mathrm{C}$. YPD liquid medium was used for routine growth of strains. For selection of strains containing the $S A T 1$-flipper cassette (156), nourseothricin $(200 \mu \mathrm{g} / \mathrm{ml})$ was added to YPD agar plates. One Shot Escherichia coli TOP10 chemically competent cells (Invitrogen, Carlsbad, CA) were used as the host for plasmid construction and propagation. These strains were grown in Luria-Bertani (LB) broth or on LB agar plates supplemented with 100_g/ml ampicillin (Sigma) or $50 \mu \mathrm{g} / \mathrm{ml}$ kanamycin (Fisher BioReagents, Fair Lawn, NJ) when required.

\section{qRT-PCR}

First-strand cDNAs were synthesized separately from $1 \mu \mathrm{g}$ of total RNA in a $21-\mu 1$ reaction mixture volume using the SuperScript first-strand synthesis system for reverse transcription (Invitrogen). Quantitative PCRs (qRT-PCRs) were performed in triplicate as technical replicates using the 7000 sequence detection system (Applied Biosystems). PCRs were performed, independently amplifying 18S rRNA or the ACT1gene (normalizing genes) and the genes of interest (GOI) from the same cDNA, using SYBR green PCR master mix (Applied Biosystems). Genespecific primers were designed using Primer Express software (Applied Biosystems) synthesized by Integrated DNA Technologies (Coralville, IA) and are listed in Table 2. The PCR conditions consisted of AmpliTaq Gold activation at $95^{\circ} \mathrm{C}$ for $10 \mathrm{~min}$, followed by 40 cycles of denaturation at $95^{\circ} \mathrm{C}$ for $15 \mathrm{~s}$ and annealing/extension at $60^{\circ} \mathrm{C}$. Determination of the dissociation curve and detection of SYBR green fluorescence was performed by using software provided with the 7000 sequence detection system. The cycle threshold $(C T)$ value of the normalizing gene was subtracted from the GOI to obtain the $\triangle C T$ value. The $\triangle C T$ value of a calibrator was subtracted from the sample $\Delta C T$ value to obtain the $\Delta \Delta C T$ value. The change in fold expression was obtained by calculating $2^{-\Delta \Delta C T}$. The standard error was calculated from $\triangle C T$ values as previously described (44).

\section{Plasmid construction for allele sequencing}

C. albicans UPC2 coding sequences were amplified by PCR ( $P f u$ DNA polymerase; Stratagene) from $C$. albicans genomic DNA using the primers UPC2-A and UPC2-E

(Table 2.2). Products were cloned into pCR-BLUNTII-TOPO using a Zero Blunt TOPO 


\begin{tabular}{|c|c|c|c|}
\hline Strain & $\begin{array}{c}\text { Strain } \\
\text { background }\end{array}$ & $\begin{array}{c}\text { Relevent characteristics or } \\
\text { genotype }\end{array}$ & Source \\
\hline SC5314 & $\mathrm{N} / \mathrm{A}$ & $U P C 2-1 / U P C 2-2$ & ATCC \\
\hline Clinical isolates $(\# 1-\# 10)$ & $\mathrm{N} / \mathrm{A}$ & azole-susceptible & University of Iowa \\
\hline Clinical isolates (\#11-\#72) & N/A & azole-resistant & University of Iowa \\
\hline \multicolumn{4}{|c|}{ Constructed laboratory strains } \\
\hline UPC2M4A & SC5314 & 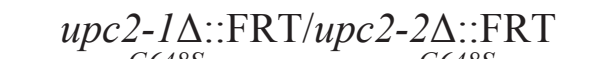 & Dunkel, 2008 \\
\hline $11 \mathrm{~A} 8 \mathrm{~A} 2 \mathrm{~A}$ & UPC2M4A & $U P C 2^{G 648 S}::$ FRT/UPC $2^{G 648 S}::$ FRT & This study \\
\hline $\mathrm{SC} 11 \mathrm{~A} 1 \mathrm{~A}$ & SC5314 & $U P C 2 / U P C 2^{G 648 S}:: \mathrm{FRT}$ & This study \\
\hline $22 \mathrm{~A} 1 \mathrm{~A} 13 \mathrm{~A}$ & UPC2M4A & $U P C 2^{G 304 R}::$ FRT/UPC2 $2^{G 304 R}::$ FRT & This study \\
\hline $\mathrm{SC} 22 \mathrm{~A} 3 \mathrm{~A}$ & SC5314 & $U P C 2 / U P C 2^{G 304 R}::$ FRT & This study \\
\hline YFA3A2K1 & UPC2M4A & $U P C 2^{Y 642 F}::$ FRT/UPC $2^{Y 642 F}::$ FRT & This study \\
\hline SCYFA2A & SC5314 & $U P C 2 / U P C 2^{Y 642 F}::$ FRT & This study \\
\hline AVA1A16A & UPC2M4A & $U P C 2^{A 646 V}::$ FRT/UPC $2^{A 646 V}::$ FRT & This study \\
\hline SCAVA4 & SC5314 & UPC2/UPC $2^{A 646 V}:: S A T 1-F L I P$ & This study \\
\hline 25B2D1 & UPC2M4A & $U P C 2^{W 478 C}::$ FRT/UPC2 $2^{W 478 C}::$ FRT & This study \\
\hline SC25A1 & SC5314 & $U P C 2 / U P C 2^{W 478 C}::$ FRT & This study \\
\hline $28 \mathrm{~A} 7 \mathrm{~A} 10 \mathrm{~A}$ & UPC2M4A & $U P C 2^{A 643 V}::$ FRT/UPC $2^{A 643 V}::$ FRT & This study \\
\hline SC28A3A & SC5314 & $U P C 2 / U P C 2^{A 643 V}:: \mathrm{FRT}$ & This study \\
\hline SCUPC2R12A & SC5314 & $U P C 2^{G 648 D}:: \mathrm{FRT} / U P C 2-2$ & Heilmann, 2010 \\
\hline SCUPC2R14A & SC5314 & $U P C 2^{G 648 D}::$ FRT/UPC2 $2^{G 648 D}:: F R T$ & Heilmann, 2010 \\
\hline SCUPC2R32A & SC5314 & $U P C 2^{A 643 T}:: \mathrm{FRT} / U P C 2-2$ & Heilmann, 2010 \\
\hline SCUPC2R34A & SC5314 & $U P C 2^{A 643 T}::$ FRT/UPC $2^{A 643 T}::$ FRT & Heilmann, 2010 \\
\hline
\end{tabular}


Table 2.2. Primers used in this study.

\begin{tabular}{|c|c|}
\hline Primer & Sequence \\
\hline \multicolumn{2}{|l|}{ qRT-PCR } \\
\hline$A C T 1-\mathrm{F}$ & 5'-ACGGTGAAGAAGTTGCTGCTTTAGTT-3' \\
\hline$A C T 1-\mathrm{R}$ & 5'-CGTCGTCACCGGCAAAA-3' \\
\hline $18 S-\mathrm{F}$ & 5'-CACGACGGAGTTTCACAAGA-3' \\
\hline $18 S-\mathrm{R}$ & 5'-CGATGGAAGTTTGAGGCAAT-3' \\
\hline ERG11-F & 5'-ССССТАТTAATTTTGTTTTCССТАATTTAC-3' \\
\hline ERG11-R & 5'-CACGTTCTCTTCTCAGTTTAATTTCTTTC-3' \\
\hline$C D R 1-\mathrm{F}$ & 5'-ATTCTAAGATGTCGTCGCAAGATG-3' \\
\hline CDR1-R & 5'-AGTTCTGGCTAAATTCGTAATGTTTTC-3' \\
\hline$C D R 2-\mathrm{F}$ & 5'-TAGTCCATTCAACGGCAACAAT-3' \\
\hline$C D R 2-\mathrm{R}$ & 5'-CACCCAGTATTTGGCATTGAAA-3' \\
\hline$B M R 1-\mathrm{F}$ & 5'-ACATAAATACTTTGCCCATCCAGAA-3' \\
\hline$B M R 1-\mathrm{R}$ & 5'-AAGAGTTGGTTTGTAATCGGCTAAA-3' \\
\hline \multicolumn{2}{|l|}{$\begin{array}{l}U P C 2 \text { mutant } \\
\text { construction }\end{array}$} \\
\hline$U P C 2-\mathrm{A}$ & 5'-GGGCCCGAGATCTTGATGTCATTAG-3' \\
\hline$U P C 2-\mathrm{B}$ & 5'-CTCGAGCTATATCTTCAATGAACTG-3' \\
\hline$U P C 2-\mathrm{E}$ & 5'-CTCGAGCACCACAGTAACGAATCAC-3' \\
\hline$U P C 2-\mathrm{C}$ & 5'-CCGCGGACAGGTCAATACCGCGTAG-3' \\
\hline$U P C 2-\mathrm{D}$ & 5'-GAGCTCGTTCCTCTAGTATCACTCTT-3' \\
\hline \multicolumn{2}{|c|}{$U P C 2$ sequencing } \\
\hline$U P C 2$ seqA & 5'-CTGCAGAGAATCACAGTGAAGTTC-3' \\
\hline$U P C 2$ seqB & 5'-CTCAGCCGGTGATTCCTCCA-3' \\
\hline$U P C 2 \mathrm{seq} C$ & 5'-CGGTCAAACCTCAATATGCTTGAC-3' \\
\hline$U P C 2$ seqD & 5'-GTTTCCAGTGCTTTTGGACTCTCC-3' \\
\hline$U P C 2$ seqZ & 5'-CCTATCATCTACGCGGTATTGACC-3' \\
\hline$U P C 2 \mathrm{seq} F$ & 5'-TGGAGGAATCACCGGCTGAG-3' \\
\hline$U P C 2$ seqG & 5'-GTCAAGCATATTGAGATTTGACCG-3' \\
\hline$U P C 2 \mathrm{seq} H$ & 5'-GGAGAGTCCAAAAGCACTGGAAAC-3' \\
\hline
\end{tabular}

Underlined sequence reflects the introduction of a restriction site sequence. 
PCR cloning kit (Invitrogen) and transferred into Escherichia coli TOP10 cells with selection on LB agar plates containing $50 \mu \mathrm{g} / \mathrm{ml}$ kanamycin. Plasmid DNA was purified (QIAprep; Qiagen, Germantown, MD) and sequenced on an ABI model 3130XL genetic analyzer using the UPC2 sequencing primers (Table 2.2), resulting in a full-length sequence from both strands of the C. albicans UPC2 gene. The sequencing was performed using six sets of clones derived from three independent PCRs for each strain/isolate sequenced.

Sequenced plasmids containing the UPC2 open reading frame (ORF) whose predicted translation indicated an amino acid substitution were digested with restriction enzymes ApaI and XhoI, which excised the fulllength ORF from the plasmid, and the UPC2 alleles were cloned upstream of the $S A T 1$-flipper cassette into the ApaI and XhoI sites of plasmid pSFS2 (25). The UPC2 downstream segments were amplified with Ex Taq (TaKaRa) using primers UPC2C and UPC2D and cloned downstream of the SAT1-flipper cassette in pSFS2 using the SacI and SacII sites. This process generated plasmids pUPC2-G648S, pUPC2-M597I, pUPC2- G304R, pUPC2-A643V, pUPC2-Y642F, and pUPC2-A646V.

\section{Construction of $U P C 2$ allele strains}

C. albicans strains UPC2M4A (7) and SC5314 were transformed by electroporation with gel-purified inserts from plasmids pUPC2-G648S, pUPC2-M597I, pUPC2-G304R, pUPC2-A643V, pUPC2-Y642F, pUPC2-W467C, and pUPC2-A646V derived from the plasmid pSFS2. pSFS2 contains the SAT1-flipper disruption cassette developed by Reuss et al. (156), consisting of the $S A T 1$ selectable marker which confers resistance to nourseothricin and the FLP flipper recombinase gene, both flanked by FRT sites (flipper recombinase target sequences). Nourseothricin-resistant transformants were selected as previously described (156). Upon induction of the FLP gene, the cassette is excised such that only the UPC2 allele with a downstream FRT is left in the UPC2 locus. Integration of constructs was confirmed by Southern hybridization.

\section{Fluconazole susceptibility testing}

MICs were obtained by using a modified CLSI protocol outlined in CLSI document M27-A3 using RPMI or YPD medium. Overnight cultures grown at $30^{\circ} \mathrm{C}$ were streaked onto Sabouraud's agar. Plated cultures were grown for $24 \mathrm{~h}$ at $30^{\circ} \mathrm{C}$. Individual colonies were suspended in sterile water until anOD600 of 0.1 was reached. The working colony concentration was made by making a 1:50 dilution and a 1:20 dilution sequentially in medium. Aliquots of $100 \mu \mathrm{l}$ from the working stock were used to inoculate a series of fluconazole-YPD medium dilutions, the highest being $64 \mu \mathrm{g} / \mathrm{ml}$. Similar procedures were used for terbinafine and amphotericin B dilutions; however, the highest concentration used for these agents was $8 \mu \mathrm{g} / \mathrm{ml}$. Cultures were incubated at $35^{\circ} \mathrm{C}$ for $48 \mathrm{~h}$, and MICs were recorded. Azole MICs for constructed strains were obtained by Etest (bioMérieux) following the manufacturer's instructions. Briefly, yeast cultures were grown in YPD 
broth medium overnight at $30^{\circ} \mathrm{C}$ in a shaking incubator and plated to Sabouraud dextrose agar at $18 \mathrm{~h}$. Isolated colonies were then selected from these plated cultures, suspended in deionized water to achieve $0.5 \mathrm{McF}$ arland turbidity, and streaked evenly across YPD plates by rotating $90^{\circ}$ in three directions. Etest strips were then applied to the inoculated agar surface, and MICs were read at both 24 and $48 \mathrm{~h}$.

\section{Ergosterol quantification analysis}

Ergosterol was extracted and quantified as described previously (100). Briefly, a single colony of $C$. albicans from a freshYPDplate was used to inoculate $50 \mathrm{ml}$ of RPMI 1640 plus 2\% glucose (Sigma, St. Louis, MO) and incubated for $16 \mathrm{~h}$ with shaking at $35^{\circ} \mathrm{C}$. Stationary-phase cells were collected by centrifugation for $5 \mathrm{~min}$ at 2,700 rpm and washed twice with sterile distilled water. The net weight of the pellet was determined. To each pellet, $3 \mathrm{ml}$ of $25 \%$ alcoholic potassium hydroxide solution $(25 \mathrm{~g}$ of $\mathrm{KOH}, 35 \mathrm{ml}$ of sterile distilled water, with final volume adjusted to $100 \mathrm{ml}$ with $100 \%$ ethanol) was added, and then the pellet was vortexed for $1 \mathrm{~min}$. Cell suspensions were transferred to a sterile borosilicate glass screw-cap tube and incubated at $85^{\circ} \mathrm{C}$ for $1 \mathrm{~h}$. Sterols were then extracted from cooled tubes by the addition of a mixture of $3 \mathrm{ml} n$-heptane and $1 \mathrm{ml}$ sterile distilled water followed by vortexing for $3 \mathrm{~min}$. The heptane layer was transferred to a clean sterile borosilicate glass tube and stored at $20^{\circ} \mathrm{C}$ for up to $24 \mathrm{~h}$.A $100-\mu \mathrm{l}$ amount of the sterol-heptane mixture was scanned spectrophotometrically between $240 \mathrm{~nm}$ and $300 \mathrm{~nm}$ with a DU530 life science UV spectrophotometer (Beckman Coulter, La Brea, CA). The presence of ergosterol in the extracted sample resulted in a characteristic fourpeaked curve. The absence of detectible ergosterol was indicated by a flat line. All samples were blanked to $n$-heptane. A decrease in the height of the absorbance peaks correlates to a decrease in ergosterol content. Statistical significance was determined by using Student's $t$ test $(P<0.05)$.

\section{Transcriptional profiling}

Gene expression profiles were obtained by hybridizing labeled cRNAs generated from $C$. albicans total RNA onto Affymetrix $C$. albicans custom expression arrays (CAN07, 49-5241 array format), which have been described previously (127). Total RNA was isolated as described earlier and subsequent cRNA synthesis/labeling, as well as probe hybridization, array scanning, and data analysis, were performed as previously described (127).

Among direct comparisons between strains, genes were considered to be differentially expressed if their change in expression was at least 1.5 -fold greater (for upregulated genes) or at least 1.5-fold less (for downregulated genes) in both independent experiments of each comparison. Cells that were empty were called "absent" by the Affymetrix criteria for the corresponding comparison/experiment, and thus, their expression values were not valid. Examination of gene ontology $(\mathrm{GO})$ classification 
enrichment of microarray data was performed using the CGD gene ontology term finder (http://www.candidagenome.org/cgi-bin/GO/goTermFinder).

\section{Accession numbers}

The coding sequences of the UPC2 alleles described in this study have been deposited in GenBank with the following accession numbers: JX494823, JX494820, JX494821, JX494822, JX494818, JX494819, JX494816, JX494817, JX494824, and JX494825. Data files for each scanned Affymetrix microarray chip were submitted to the Gene Expression Omnibus database (Chalfont, United Kingdom) and given the accession number GSE39201.

\section{RESULTS}

\section{Expression of $E R G 11, C D R 1, C D R 2$, and $M D R 1$ in 62 fluconazole-resistant clinical isolates of $C$. albicans}

In order to examine the prevalence of and identify possible UPC2 GOF mutations, I obtained a collection of 72 clinical C. albicans isolates from the laboratory of Daniel Diekema (University of Iowa). All isolates were unrelated, and they were collected from both oral and nonoral sources, including blood, tissue, and abdominal fluid (see Appendix A table). Fluconazole susceptibilities were confirmed in our laboratory. I defined all isolates with a MIC of $\geq 16 \mu \mathrm{g} / \mathrm{ml}$ as having resistance to fluconazole for the purpose of this study. From our collection of 72 isolates, 9 were classified as fluconazole susceptible, while the remaining isolates were resistant. We then measured the levels of expression of ERG11,CDR1,CDR2, and MDR1 for all isolates in order to determine the relative prevalence of overexpression of genes indicative of specific mechanisms of azole resistance (Figure 2.1). I defined overexpression as an increase in expression of at least 2-fold. I observed that 77\% $(n=49)$ of resistant isolates overexpressed both CDR1 and $C D R 2$. MDRl overexpression was increased by at least 2 -fold in $76 \%(n=48)$ of resistant isolates, but only $21 \%(n=13)$ expressed $M D R 1$ at levels consistent with the levels observed in isolates with MDRl-mediated fluconazole resistance, in which MDRI expression is substantially increased. For example, in a study by Morschhäuser et al., the clinical isolates examined exhibited 400- to 1,200-fold increases in MDRI expression.(127) Surprisingly, ERG11 was found to be overexpressed in $75 \%(n=47)$ of fluconazole-resistant isolates in this collection.

\section{Most but not all isolates that overexpress ERG11 carry a GOF mutation in UPC2}

Twenty-nine of the 47 isolates that overexpressed ERG11 possessed one or both $U P C 2$ alleles with a mutation that resulted in a predicted amino acid substitution. 
Figure 2.1. Expression levels of $C D R 1, C D R 2, M D R 1$, and $E R G 11$ in 63 fluconazole-resistant clinical isolates.

All gene expression levels were measured by qRT-PCR in triplicate, and fold expression of genes in resistant isolates was compared to the average of the expression levels in three susceptible isolates. Results for 63 isolates with reduced susceptibility to fluconazole are represented, but only even-numbered isolates' results are labeled. Error bars show standard errors. 

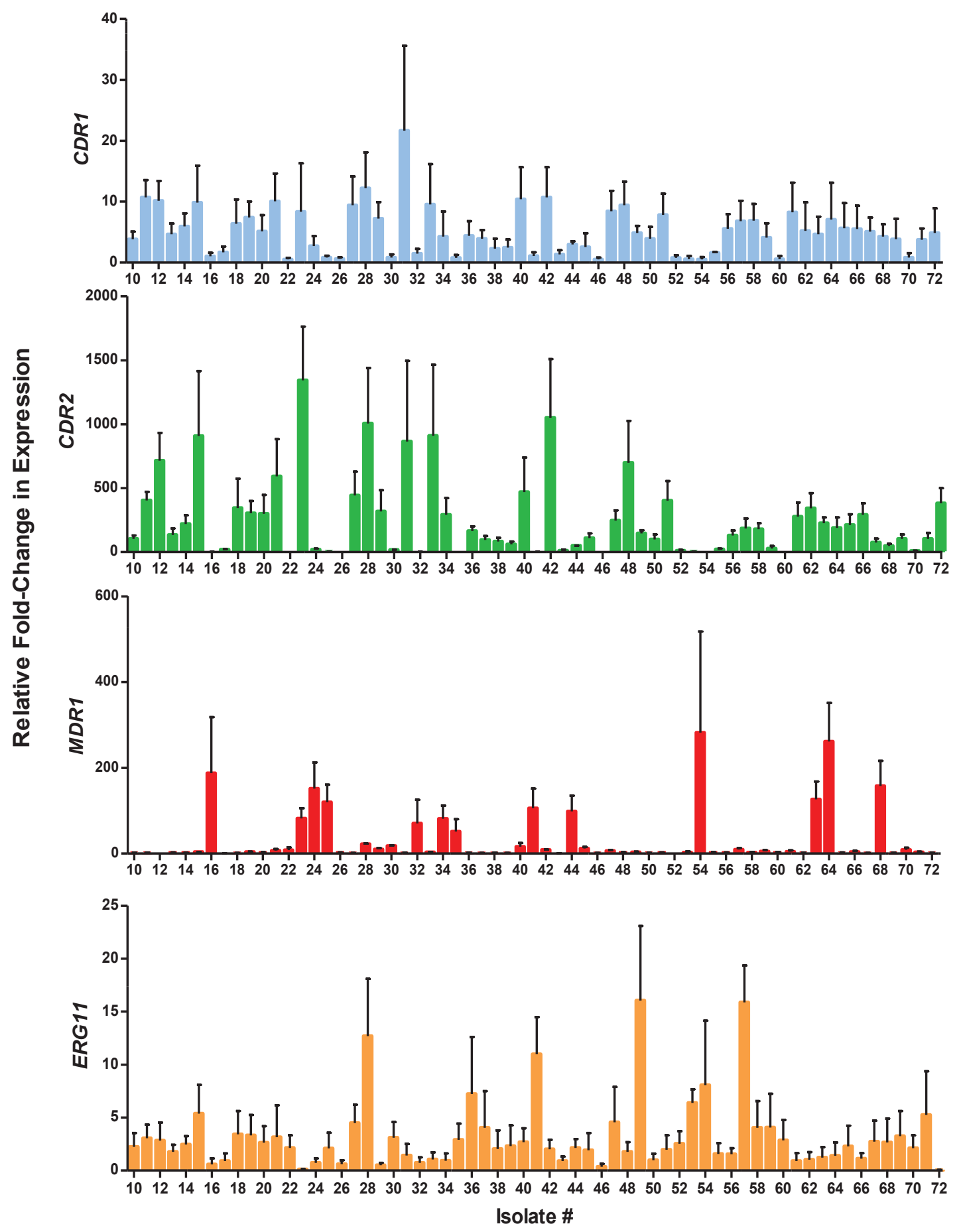

MIC $(\mu \mathrm{g} / \mathrm{mL})$

32

64

128

256 
Although many silent mutations were observed in the UPC2 alleles tested, eight distinct single $U P C 2$ mutations were also recovered, three of which had been described previously: G648D, A643T, and A643V (Table 2.3). Eighteen clinical isolates were found to overexpress $E R G 11$ yet contained no amino acid substitution in Upc2. These data suggest an alternate mechanism of activating ERG11 expression in these isolates.

\section{GOF mutations in $U P C 2$ result in increased expression of $E R G 11$}

In order to assess the contribution of each individual mutant $U P C 2$ allele to fluconazole resistance, I expressed each mutant allele alone, with the other UPC2 allele disrupted (data not shown), as a heterozygote with a wild-type UPC2 allele, and as a homozygote. ERG11 mRNA abundance was measured by qRT-PCR (Figure 2.2). The homozygous null upc $2 \Delta$ strain showed a significant decrease in $E R G 11$ expression compared to that of its parental wild-type strain. Seven of the eight mutations tested resulted in increased ERG11 expression, with the strongest expression observed among homozygous strains. Interestingly, different mutations elicited different levels of ERG11 expression, with the G648D substitution resulting in the highest level of expression.

\section{GOF mutations inUPC2 result in elevated cellular ergosterol content}

In order to confirm that activation of Upc2 results in a global increase of ergosterol biosynthesis, I compared the cellular ergosterol content in the wild type to the amounts in derivatives that were heterozygous or homozygous for various UPC2 mutations. I found that strains carrying $U P C 2 \mathrm{GOF}$ alleles generally contained larger amounts of ergosterol than the wild-type strain (Figure 2.3). For many isolates, increased ergosterol levels correlated with the increased ERG11 transcript levels resulting from each individual $U P C 2$ mutation; however, this was not the case for the A643V allele, which resulted in strong ERG11 expression but a nonsignificant increase in cellular ergosterol content. $U P C 2$ alleles containing the G648D, W478C, and Y642F amino acid substitutions showed statistically significant increases in ergosterol content relative to the amount in the wild-type strain, while strains carrying other UPC2 GOF alleles trended toward larger amounts of cellular ergosterol.

\section{GOF mutations in UPC2 influence susceptibility to antifungals}

To examine the impact of distinct $U P C 2$ mutations on antifungal resistance, I determined the susceptibilities of strains constructed to carry mutant $U P C 2$ alleles to a panel of antifungals (Table 2.4). Included in this panel were agents in the azole class (fluconazole, itraconazole, and voriconazole). Also included were terbinafine, which inhibits another ergosterol biosynthesis enzyme (squalene epoxidase), and amphotericin $\mathrm{B}$, which targets ergosterol in the fungal cell membrane. All strains constructed were

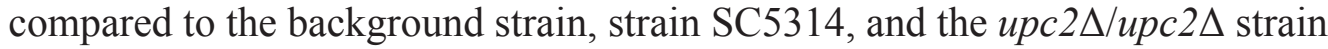


Table 2.3. Occurrence of Upc2 amino acid substitutions in predicted translated sequence in fluconazole resistant clinical $E R G 11$-overexpressing isolates.

\begin{tabular}{|c|c|c|c|c|c|c|c|}
\hline \multirow[b]{2}{*}{$\begin{array}{c}\text { Upc2 } \\
\text { substitution }\end{array}$} & \multirow[b]{2}{*}{ Isolate } & \multirow[b]{2}{*}{ Genotype } & \multirow[b]{2}{*}{$\begin{array}{c}\text { Fluconazole } \\
\text { MIC } \\
(\mu \mathrm{g} / \mathrm{mL})\end{array}$} & \multicolumn{4}{|c|}{ Expression of efflux transporter genes ${ }^{\mathbf{a}}$} \\
\hline & & & & $\begin{array}{c}\text { ERG11 } \\
( \pm \mathrm{SE})\end{array}$ & $\begin{array}{l}C D R 1 \\
( \pm \mathrm{SE})\end{array}$ & $\begin{array}{l}C D R 2 \\
( \pm \mathrm{SE})\end{array}$ & $\begin{array}{c}\text { MDR1 } \\
( \pm \mathrm{SE})\end{array}$ \\
\hline \multirow[t]{6}{*}{ G648D } & 21 & Heterozygous & 32 & $6.2( \pm 0.2)$ & $14.7( \pm 5.5)$ & $883.3( \pm 301.9)$ & $10.4( \pm 3.1)$ \\
\hline & 38 & Heterozygous & 64 & $3.8( \pm 0.3)$ & $3.9( \pm 0.7)$ & $111.0( \pm 57.1)$ & $1.4( \pm 0.2)$ \\
\hline & 47 & Heterozygous & 128 & $7.9( \pm 1.2)$ & $11.8( \pm 5.1)$ & $327.0( \pm 166.3)$ & $8.2( \pm 3.1)$ \\
\hline & 52 & Heterozygous & 256 & $3.7( \pm 1.4)$ & $1.2( \pm 0.3)$ & $17.1( \pm 3.2)$ & $0.3( \pm 0.1)$ \\
\hline & 59 & Heterozygous & $>256$ & $7.2( \pm 1.0)$ & $6.5( \pm 0.3)$ & $48.5( \pm 0.3)$ & $8.0( \pm 0.2)$ \\
\hline & 71 & Heterozygous & $>256$ & $9.4( \pm 1.1)$ & $5.6( \pm 1.9)$ & $149.8( \pm 56.5)$ & $4.6( \pm 1.8)$ \\
\hline \multirow[t]{9}{*}{ G648S } & 25 & Heterozygous & 64 & $3.6( \pm 0.6)$ & $1.1( \pm 0.5)$ & $2.8( \pm 1.1)$ & $160.5( \pm 78.9)$ \\
\hline & 35 & Heterozygous & $>256$ & $4.4( \pm 1.3)$ & $1.2( \pm 0.4)$ & $0.7( \pm 0.3)$ & $79.9( \pm 22.8)$ \\
\hline & 55 & Homozygous & $>256$ & $2.6( \pm 0.6)$ & $1.7( \pm 1.5)$ & $26.2( \pm 17.4)$ & $0.9( \pm 3.2)$ \\
\hline & 56 & Homozygous & $>256$ & $2.1( \pm 1.0)$ & $8.0( \pm 3.1)$ & $167.8( \pm 94.0)$ & $2.5( \pm 0.9)$ \\
\hline & 57 & Homozygous & $>256$ & $19.4( \pm 12.4)$ & $10.2( \pm 3.5)$ & $263.3( \pm 106.0)$ & $12.9( \pm 5.2)$ \\
\hline & 58 & Homozygous & $>256$ & $6.6( \pm 1.5)$ & $9.7( \pm 4.2)$ & $226.6( \pm 131.7)$ & $3.8( \pm 1.2)$ \\
\hline & 65 & Heterozygous & $>256$ & $4.2( \pm 0.3)$ & $9.8( \pm 1.5)$ & $294.9( \pm 128.4)$ & $2.4( \pm 0.1)$ \\
\hline & 67 & Heterozygous & $>256$ & $4.7( \pm 0.7)$ & $7.4( \pm 2.8)$ & $105.1( \pm 43.3)$ & $1.2( \pm 0.5)$ \\
\hline & 69 & Heterozygous & $>256$ & $5.6( \pm 0.9)$ & $7.2( \pm 0.5)$ & $136.8( \pm 69.3)$ & $1.4( \pm 0.5)$ \\
\hline A643T & 13 & Homozygous & 16 & $2.4( \pm 1.1)$ & $6.4( \pm 2.9)$ & $185.9( \pm 80.9)$ & $2.4(1.6)$ \\
\hline \multirow[t]{2}{*}{ A643V } & 30 & Heterozygous & 64 & $4.6( \pm 1.6)$ & $1.4( \pm 0.3)$ & $18.0( \pm 15.7)$ & $18.9( \pm 16.7)$ \\
\hline & 68 & Heterozygous & $>256$ & $4.9( \pm 0.4)$ & $6.3( \pm 2.2)$ & $64.5( \pm 31.8)$ & $216.3( \pm 98.9)$ \\
\hline
\end{tabular}


Table 2.3. Continued.

\begin{tabular}{|c|c|c|c|c|c|c|c|}
\hline \multirow[b]{2}{*}{$\begin{array}{c}\text { Upc2 } \\
\text { Substitution }\end{array}$} & \multirow[b]{2}{*}{ Isolate } & \multirow[b]{2}{*}{ Genotype } & \multirow{2}{*}{$\begin{array}{c}\text { Fluconazole } \\
\text { MIC } \\
(\mu \mathrm{g} / \mathrm{mL})\end{array}$} & \multicolumn{4}{|c|}{ Expression of efflux transporter genes ${ }^{a}$} \\
\hline & & & & ERG11 ( \pm SE) & $C D R 1( \pm \mathrm{SE})$ & $C D R 2( \pm \mathrm{SE})$ & $M D R 1( \pm \mathrm{SE})$ \\
\hline \multirow[t]{2}{*}{$\mathrm{A} 646 \mathrm{~V}$} & 45 & Heterozygous & 128 & $3.5( \pm 0.3)$ & $4.8( \pm 0.3)$ & $147.8( \pm 71.7)$ & $15.5( \pm 7.7)$ \\
\hline & 67 & Heterozygous & $>256$ & $4.7( \pm 0.7)$ & $7.4( \pm 2.8)$ & $105.1( \pm 43.3)$ & $1.2( \pm 0.5)$ \\
\hline \multirow[t]{2}{*}{ Y642F } & 33 & Homozygous & 36 & $12.6( \pm 1.8)$ & $6.8( \pm 2.0)$ & $201.5( \pm 129.0)$ & $1.5( \pm 0.5)$ \\
\hline & 34 & Homozygous & 37 & $7.5( \pm 0.6)$ & $5.4(2.5)$ & $126.8( \pm 62.6)$ & $1.2( \pm 0.5)$ \\
\hline \multirow[t]{3}{*}{ W478C } & 15 & Heterozygous & 16 & $8.1( \pm 2.7)$ & $15.9( \pm 3.8)$ & $\begin{array}{c}1415.1 \\
( \pm 400.1)\end{array}$ & $4.0( \pm 3.0)$ \\
\hline & 27 & Heterozygous & 64 & $6.2( \pm 2.8)$ & $14.2( \pm 4.7)$ & $629.7( \pm 255.3)$ & $1.1( \pm 0.1)$ \\
\hline & 28 & Heterozygous & 64 & $18.1( \pm 7.3)$ & $18.1( \pm 6.3)$ & $\begin{array}{c}1439.7 \\
( \pm 570.8)\end{array}$ & $23.2( \pm 20.6)$ \\
\hline \multirow[t]{2}{*}{ G304R } & 18 & Heterozygous & 32 & $5.6( \pm 1.2)$ & $10.4( \pm 2.4)$ & $575.0( \pm 117.2)$ & $1.0( \pm 0.3)$ \\
\hline & 19 & Heterozygous & 32 & $5.2( \pm 1.4)$ & $10.0( \pm 4.8)$ & $401.3( \pm 204.5)$ & $4.6( \pm 3.0)$ \\
\hline
\end{tabular}

a q-RT-PCR was performed in triplicate. (SE) is calculated standard error. 


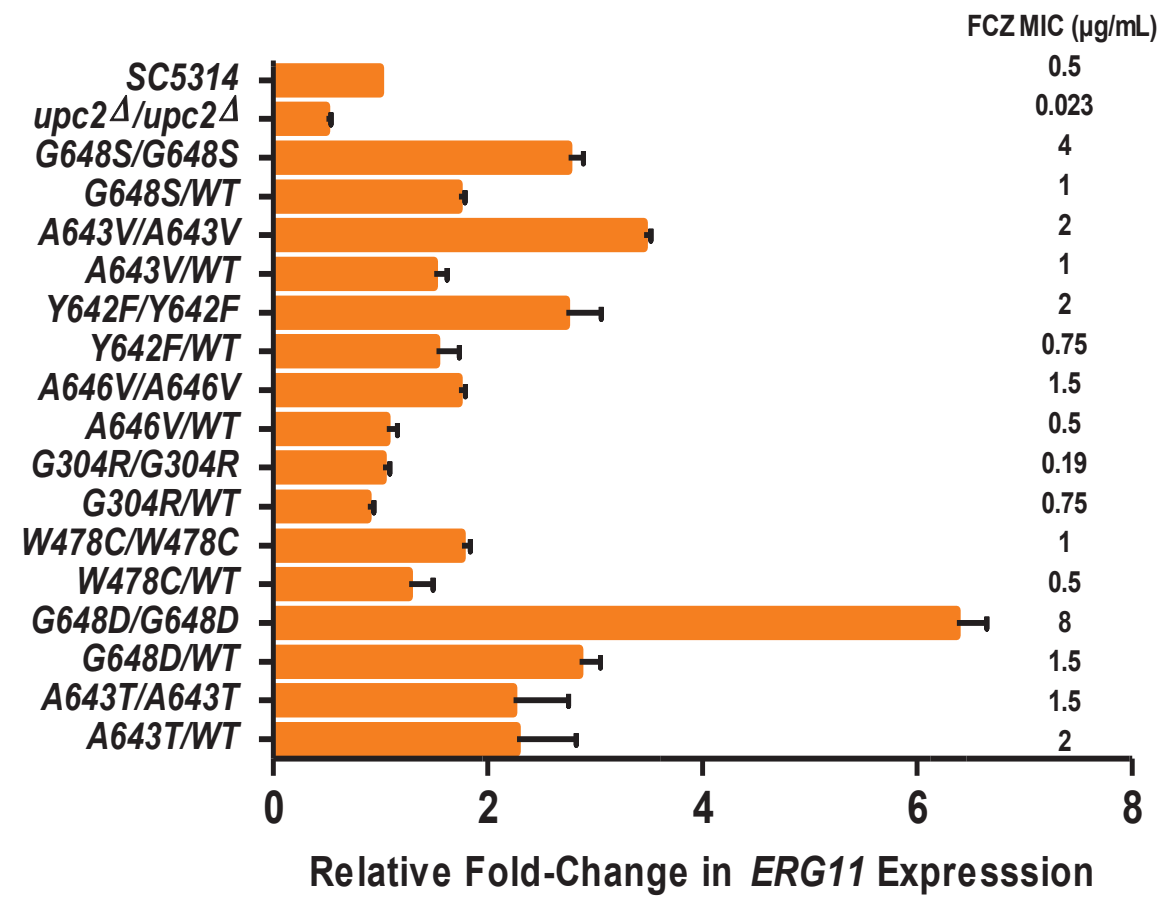

Figure 2.2. $\quad E R G 11$ transcription levels in strains carrying mutant $U P C 2$ alleles are represented as averages from three independent $q R T-P C R$ assays.

Error bars show standard errors. ERG11 expression was quantified for each mutant strain listed and is compared to that of wild-type strain SC5314. 
Figure 2.3. Ergosterol quantification in $C$. albicans laboratory strains expressing mutant $U P C 2$ alleles.

Increased ergosterol content was shown for strains expressing 2 UPC2 mutant alleles compared to the expression levels in heterozygote and wild-type strains. The heptane extraction layer from 16-h cultures was scanned spectrophotometrically between 240 and $300 \mathrm{~nm}$. The presence of ergosterol in an extracted sample resulted in a 4-peak curve. A decrease in absorbance peaks correlates to a decrease in ergosterol content. Each panel represents the results for a different Upc2 GOF mutation, as follows: G648S (A), A643V (B), Y642F (C), G648D (D), A646V (E), A643T (F), W478C (G), and G304R (H).UPC2 mutant alleles were expressed as homozygotes with the same GOF mutation $(\mathrm{O})$ or as heterozygotes with the wild-type allele $(\boldsymbol{\Delta})$. Their absorbencies were compared to those of the wild-type strain SC5314 (•). The results for the homozygous revertants shown in panels $\mathrm{C}, \mathrm{D}$, and $\mathrm{G}$ showed statistically significant greater ergosterol contents than were found in the wild type $(\mathrm{P}<0.05)$. 

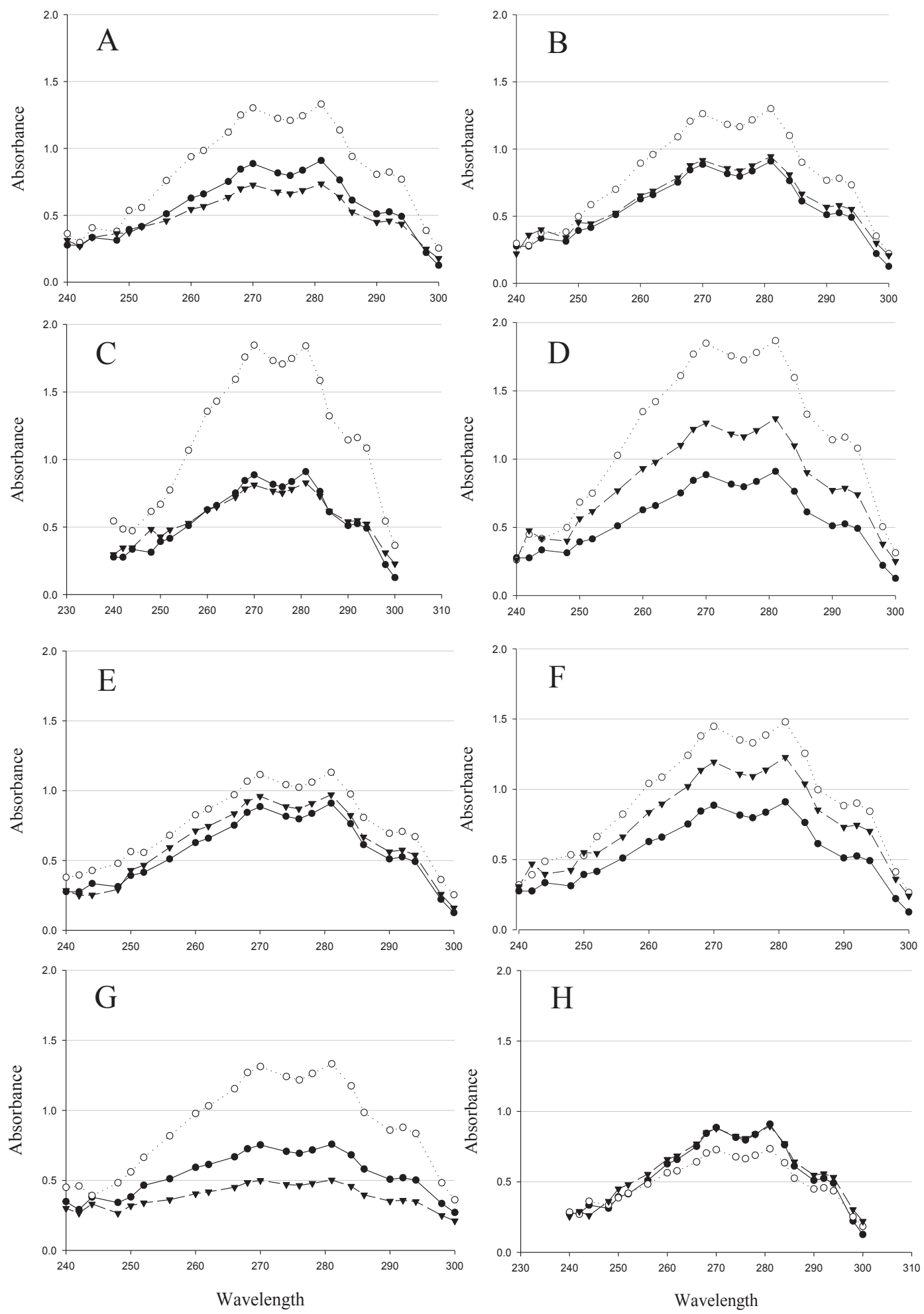
Table 2.4. MICs of strains expressing UPC2 mutant alleles to a panel of antifungals in YPD.

\begin{tabular}{|c|c|c|c|c|c|c|c|c|c|c|c|}
\hline \multirow[b]{2}{*}{ Strain } & \multirow[b]{2}{*}{ Relevant genotype } & \multicolumn{2}{|c|}{$\mathrm{FCZ}(\mu \mathrm{g} / \mathrm{mL})$} & \multicolumn{2}{|c|}{$\mathrm{ICZ}(\mu \mathrm{g} / \mathrm{mL})$} & \multicolumn{2}{|c|}{$\underline{\mathrm{VCZ}}(\mu \mathrm{g} / \mathrm{mL})$} & \multicolumn{2}{|c|}{ TBF $(\mu \mathrm{g} / \mathrm{mL})$} & \multicolumn{2}{|c|}{ AMB $(\mu \mathrm{g} / \mathrm{mL})$} \\
\hline & & $24 \mathrm{hr}$ & $48 \mathrm{hr}$ & $24 \mathrm{hr}$ & $48 \mathrm{hr}$ & $24 \mathrm{hr}$ & $48 \mathrm{hr}$ & $24 \mathrm{hr}$ & $48 \mathrm{hr}$ & $24 \mathrm{hr}$ & $48 \mathrm{hr}$ \\
\hline SC5314 & $U P C 2-1 / U P C 2-2$ & 0.5 & 4 & 0.016 & 0.023 & 0.008 & 0.032 & 0.5 & 2 & 0.0313 & 0.125 \\
\hline UPC2M4A & 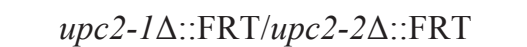 & 0.023 & 0.032 & 0.002 & 0.004 & 0.002 & 0.002 & 0.016 & 0.016 & 0.0313 & 0.5 \\
\hline UPC2M2K21A & UPC2-1S1-1-caSAT1/upc2-24::FRT & 0.25 & 1.5 & 0.012 & 0.023 & 0.004 & 0.008 & 0.25 & 1 & 0.0313 & 0.25 \\
\hline 11A8A2A & $U P C 2^{G 648 S}::$ FRT/UPC2 $2^{G 648 S}:: F R T$ & 4 & $>256^{\prime}$ & 0.5 & 0.75 & 0.25 & $>32^{\prime}$ & 2 & 4 & 0.016 & 0.125 \\
\hline SC11A1A & $U P C 2 / U P C 2^{G 648 S}::$ FRT & 1 & $>256^{\prime}$ & 0.032 & 0.19 & 0.047 & $>32^{\prime}$ & 2 & 4 & 0.0625 & 0.25 \\
\hline $28 \mathrm{~A} 7 \mathrm{~A} 10 \mathrm{~A}$ & $U P C 2^{A 643 V}::$ FRT/UPC $2^{A 643 V}::$ FRT & 2 & $>256^{\prime}$ & 0.125 & $>32$ & 0.25 & $>32^{\prime}$ & 1 & 4 & 0.016 & 0.125 \\
\hline SC28A3A & $U P C 2 / U P C 2^{A 643 V}::$ FRT & 1 & 2 & 0.023 & 0.047 & 0.047 & $>32^{\prime}$ & 1 & 2 & 0.0313 & 0.25 \\
\hline YFA3A2K1 & $U P C 2^{Y 642 F}:: \mathrm{FRT} / U P C 2^{Y 642 F}:: \mathrm{FRT}$ & 2 & $>256$ & 0.047 & 0.5 & 0.064 & $>32^{\prime}$ & 1 & 4 & 0.0625 & 0.5 \\
\hline SCYFA2A & $U P C 2 / U P C 2^{Y 642 F}::$ FRT & 0.75 & 1 & 0.023 & 0.032 & 0.023 & 0.19 & 1 & 2 & 0.0313 & 0.25 \\
\hline AVA1A16A & $U P C 2^{A 646 V}::$ FRT/UPC $2^{A 646 V}:: F R T$ & 1.5 & $>256$ & 0.064 & 0.25 & 0.064 & $>32$ & 1 & 4 & 0.0313 & 0.25 \\
\hline SCAVA4 & $U P C 2 / U P C 2^{A 646 V}:: S A T 1-F L I P$ & 0.5 & 1.5 & 0.016 & 0.032 & 0.016 & 0.064 & 1 & 2 & 0.0313 & 0.5 \\
\hline $22 \mathrm{~A} 1 \mathrm{~A} 13 \mathrm{~A}$ & $U P C 2^{G 304 R}::$ FRT/UPC2 $2^{G 304 R}::$ FRT & 0.19 & 0.75 & 0.012 & 0.023 & 0.008 & 0.032 & 1 & 2 & 0.0313 & 0.5 \\
\hline $\mathrm{SC} 22 \mathrm{~A} 3 \mathrm{~A}$ & $U P C 2 / U P C 2^{G 304 R}::$ FRT & 0.75 & $>256$ & 0.012 & 0.094 & 0.023 & $>32$ & 1 & 4 & 0.0313 & 0.25 \\
\hline 25B2D1 & $U P C 2^{W 478 C}:: \mathrm{FRT} / U P C 2^{W 478 C}:: \mathrm{FRT}$ & 1 & 2 & 0.047 & 0.047 & 0.016 & 0.5 & 0.25 & 1 & 0.0313 & 0.25 \\
\hline $\mathrm{SC} 25 \mathrm{~A} 1$ & $U P C 2 / U P C 2^{W 478 C}::$ FRT & 0.5 & 1 & 0.016 & 0.016 & 0.008 & 0.032 & 0.5 & 1 & 0.016 & 0.125 \\
\hline SCUPC2R14A & $U P C 2^{G 648 D}:: \mathrm{FRT} / U P C 2^{G 648 D}:: \mathrm{FRT}$ & 8 & $>256^{\prime}$ & $>32$ & $>32$ & 0.064 & $>32^{\prime}$ & 2 & 4 & 0.016 & 0.125 \\
\hline SCUPC2R12A & $U P C 2^{G 648 D}:: \mathrm{FRT} / U P C 2-2$ & 1.5 & $>256^{\prime}$ & 0.25 & $>32$ & 0.047 & $>32^{\prime}$ & 1 & 2 & 0.0313 & 0.25 \\
\hline SCUPC2R34A & $U P C 2^{A 643 T}:: \mathrm{FRT} / U P C 2^{A 643 T}:: \mathrm{FRT}$ & 1.5 & $>256^{\prime}$ & 2 & $>32$ & 0.064 & $>32^{\prime}$ & 2 & 2 & 0.016 & 0.125 \\
\hline SCUPC2R32A & $U P C 2^{A 643 T}::$ FRT/UPC2-2 & 2 & $>256^{\prime}$ & 0.016 & 0.032 & 0.012 & $>32^{\prime}$ & 1 & 2 & 0.016 & 0.125 \\
\hline
\end{tabular}

FCZ-fluconazole; ICZ- itraconazole; MCZ- miconazole; VCZ- voriconazole; TBF- terbinafine ; AMB- amphotericin B.

Susceptibility was tested at 24 and at 48 hours. Azole MICs were determined by Etest method while terbinafine and amphotericin B determined by broth microdilution methods. 
UPC2M4A. Strain UPC2M4A was highly susceptible to terbinafine, as well as all the azoles tested. Interestingly, this strain exhibited a 4-fold-higher MIC of amphotericin B than SC5314 at $48 \mathrm{~h}$. Strains expressing UPC2 alleles carrying the G648D, G648S, A643T, A643V, Y642F, W478C, and A646V substitutions in the predicted protein sequence all exhibited increased resistance to fluconazole compared to that of strain SC5314, irrespective of the additional presence of a wild-type allele. Strains expressing the G304R mutant allele did not show a decrease in susceptibility beyond that of the wild-type, indicating that this mutation does not result in gain of function. Correlating with increased ERG11 expression, fluconazole susceptibility also decreased, with the most prominent change occurring in the homozygous strains. A similar trend was exhibited for the other azole agents and also for terbinafine susceptibilities. For amphotericin $\mathrm{B}$, strains homozygous for an activated UPC2 mutant allele generally demonstrated a reduction in MIC at $48 \mathrm{~h}$ compared to that of the parental wild-type strain. However, all strains tested were susceptible to this agent. These results demonstrate that seven of the eight mutations in UPC2 confer increased resistance to ergosterol biosynthesis inhibitors.

\section{Genome-wide transcriptional-profile analysis of strains carrying activated $U P C 2$ alleles}

I identified genes whose expression is influenced by Upc2 by comparing the transcriptional profiles of strains that were engineered to carry one of the four GOF $U P C 2$ alleles with the strongest effects on ERG11 expression. The 11A8A2A $\left(U P C 2^{G 648 S} / U P C 2^{G 648 S}\right)$ constructed strain expressed 170 upregulated genes and 9 downregulated genes compared to the expression profile of SC5314. Strain

SCUPC2R14A (UPC2 $\left.2^{G 648 D} / U P C 2^{G 648 D}\right)$ expressed 520 upregulated and 292 downregulated genes. There were 119 genes upregulated and 81 genes downregulated for strain YFA3A2K1 (UPC2 $\left.2^{Y 642 F} / U P C 2^{Y 642 F}\right)$, while strain 28A7A10A $\left(U P C 2^{A 643 V} / U P C 2^{A 643 V}\right)$ expressed 352 upregulated and 169 downregulated genes. There were 61 commonly upregulated genes and 5 commonly downregulated genes among the four strains carrying unique UPC2 gain-of-function alleles (Tables 2.5 and 2.6). Genes commonly upregulated in all four mutant strains included those involved in ergosterol biosynthesis and in oxidoreductase activity, the major facilitator efflux pump encoded by the MDR1 gene, and the uncharacterized ATP-binding cassette transporter CDR11.

\section{DISCUSSION}

In Candida albicans, it is well established that activating mutations in transcription factors regulating genes encoding efflux pumps mediate resistance to azole antifungals. It has been shown that specific mutations in the transcription factor gene TAC1 mediate the expression of the genes encoding $\mathrm{ABC}$ transporters $C D R 1$ and $C D R 2$, resulting in increased azole resistance in C. albicans $(22,23)$. Likewise, Mrr1 has been identified as the regulator of the major facilitator superfamily (MFS) transporter Mdr1 in azoleresistant isolates, and specific mutations in this transcriptional regulator result in its 
Table 2.5. Genes upregulated by at least 1.5- fold in strains carrying UPC2 GOF alleles

\begin{tabular}{|c|c|c|c|c|c|c|c|c|c|c|}
\hline \multirow[b]{3}{*}{ Functon $^{\mathrm{a}}$} & \multirow[b]{3}{*}{ CGD name } & \multirow[b]{3}{*}{ Orf19 designation } & \multicolumn{8}{|c|}{ Change in fold expression ${ }^{b}$} \\
\hline & & & \multicolumn{2}{|c|}{ G648S } & \multicolumn{2}{|c|}{ G648D } & \multicolumn{2}{|c|}{ Y642F } & \multicolumn{2}{|c|}{ A643V } \\
\hline & & & $\operatorname{Exp} 1$ & Exp2 & Exp1 & Exp2 & Exp1 & Exp2 & Exp1 & Exp2 \\
\hline \multirow[t]{9}{*}{ Lipid metabolic process } & $E R G 1$ & orf19.406 & 1.8 & 1.8 & 2.7 & 2.3 & 2.2 & 2.0 & 2.3 & 2.3 \\
\hline & $E R G 2$ & orf19.6026 & 1.7 & 1.5 & 2.3 & 1.9 & 1.9 & 1.9 & 2.1 & 2.0 \\
\hline & $E R G 24$ & orf19.1598 & 1.7 & 1.7 & 2.3 & 2.1 & 1.7 & 1.8 & 2.1 & 2.3 \\
\hline & $E R G 27$ & orf19.3240 & 1.8 & 2.1 & 3.3 & 3.4 & 2.2 & 2.2 & 3.1 & 3.2 \\
\hline & $E R G 7$ & orf19.1570 & 1.5 & 1.6 & 2.8 & 2.1 & 1.8 & 1.6 & 2.7 & 2.4 \\
\hline & $N C P 1$ & orf19.2672 & 1.6 & 1.7 & 2.6 & 2.0 & 1.7 & 1.9 & 1.9 & 1.9 \\
\hline & & orf19.3483 & 2.0 & 2.4 & 5.3 & 4.7 & 2.9 & 2.2 & 3.5 & 3.3 \\
\hline & & orf19.1881 & 1.5 & 1.6 & 2.4 & 2.2 & 1.5 & 1.5 & 2.4 & 2.1 \\
\hline & & orf19.6025 & 2.2 & 2.1 & 3.9 & 5.2 & 3.8 & 3.1 & 5.2 & 5.2 \\
\hline Transcription factor activity & & orf19.5210 & 1.5 & 2.1 & 2.7 & 2.7 & 1.8 & 1.5 & 2.9 & 2.3 \\
\hline \multirow[t]{7}{*}{ Transport } & $M D R 1$ & orf19.5604 & 5.9 & 5.5 & 15.1 & 8.0 & 6.6 & 6.1 & 5.9 & 5.7 \\
\hline & & orf 19.5535 & 1.5 & 1.6 & 2.2 & 2.7 & 1.5 & 1.9 & 2.7 & 3.2 \\
\hline & $F R P 1$ & orf19.5634 & 5.4 & 5.7 & 17.3 & 17.5 & 8.1 & 5.9 & 16.3 & 14.7 \\
\hline & FTH1 & orf19.4802 & 2.6 & 2.6 & 5.3 & 5.2 & 2.3 & 1.9 & 6.6 & 5.2 \\
\hline & GYPl & orf19.3811 & 1.6 & 1.6 & 3.7 & 3.5 & 1.8 & 1.5 & 3.7 & 3.4 \\
\hline & & orf19.2350 & 1.7 & 1.7 & 3.4 & 4.4 & 2.0 & 1.9 & 3.2 & 3.0 \\
\hline & HXT5 & orf19.4384 & 1.8 & 2.1 & 2.6 & 2.0 & 1.8 & 1.5 & 2.3 & 1.7 \\
\hline \multirow{4}{*}{ Protein modification process } & & orf19.7329 & 1.6 & 2.1 & 3.1 & 3.0 & 1.7 & 1.8 & 2.5 & 2.3 \\
\hline & & orf 19.6025 & 2.2 & 2.1 & 3.9 & 5.2 & 3.8 & 3.1 & 5.2 & 5.2 \\
\hline & & orf 19.7547 & 1.8 & 1.5 & 2.8 & 2.7 & 2.0 & 1.5 & 3.1 & 2.3 \\
\hline & & orf 19.2285 & 1.7 & 2.5 & 1.8 & 1.5 & 1.8 & 2.4 & 2.1 & 2.1 \\
\hline Biosynthetic process & COQ4 & orf19.3008 & 1.5 & 1.5 & 2.3 & 2.8 & 1.9 & 1.6 & 2.6 & 2.5 \\
\hline
\end{tabular}


Table 2.5. Continued.

\begin{tabular}{|c|c|c|c|c|c|c|c|c|c|c|}
\hline \multirow[b]{3}{*}{ Function $^{\mathrm{a}}$} & \multirow[b]{3}{*}{ CGD name } & \multirow[b]{3}{*}{ Orf19 designation } & \multicolumn{8}{|c|}{ Change in fold expression ${ }^{b}$} \\
\hline & & & \multicolumn{2}{|c|}{ G648S } & \multicolumn{2}{|c|}{ G648D } & \multicolumn{2}{|c|}{ Y642F } & \multicolumn{2}{|c|}{ A643V } \\
\hline & & & Exp1 & Exp2 & Exp1 & Exp2 & Exp1 & Exp2 & Exp1 & $1 \quad$ Exp2 \\
\hline Biosynthetic process & HEM14 & orf19.4747 & 2.1 & 1.6 & 3.6 & 3.0 & 2.2 & 1.8 & 2.9 & 2.3 \\
\hline \multirow[t]{3}{*}{ Pathogenesis } & SLD1 & orf19.260 & 1.8 & 1.5 & 2.2 & 1.8 & 1.7 & 1.6 & 2.0 & 1.9 \\
\hline & PHO100 & orf19.4424 & 1.5 & 3.4 & 4.8 & 2.7 & 1.6 & 1.5 & 2.0 & 2.3 \\
\hline & SET3 & orf19.7221 & 2.8 & 2.7 & 6.3 & 5.7 & 4.4 & 3.6 & 4.8 & 4.8 \\
\hline \multirow[t]{8}{*}{ Metabolic process } & & orf19.3617 & 1.7 & 1.9 & 1.7 & 2.2 & 1.7 & 1.5 & 1.8 & 1.5 \\
\hline & & orf19.329 & 2.1 & 2.3 & 2.4 & 2.6 & 2.0 & 1.6 & 2.5 & 2.5 \\
\hline & & orf19.4031 & 1.8 & 1.8 & 2.5 & 2.5 & 1.6 & 1.5 & 2.9 & 2.5 \\
\hline & & orf19.6025 & 2.2 & 2.1 & 3.9 & 5.2 & 3.8 & 3.1 & 5.2 & 5.2 \\
\hline & & orf19.1865 & 2.4 & 2.2 & 3.7 & 2.9 & 2.4 & 2.2 & 3.4 & 3.1 \\
\hline & & orf19.496 & 2.4 & 2.3 & 4.4 & 3.8 & 2.5 & 2.0 & 3.6 & 3.1 \\
\hline & AROS & orf19.1237 & 1.5 & 3.4 & 5.8 & 4.2 & 1.5 & 1.9 & 2.1 & 2.8 \\
\hline & $I D P 2$ & orf19.3733 & 2.2 & 1.6 & 3.3 & 2.8 & 2.6 & 2.0 & 3.2 & 2.5 \\
\hline \multirow[t]{4}{*}{ Response to stress } & DDR48 & orf19.4082 & 2.2 & 4.3 & 5.8 & 4.7 & 2.7 & 2.9 & 3.4 & 4.3 \\
\hline & FMA1 & orf19.6837 & 1.8 & 3.2 & 2.7 & 5.3 & 1.5 & 3.3 & 1.7 & 3.0 \\
\hline & $Y M X 6$ & orf19.5713 & 1.6 & 2.6 & 2.9 & 2.0 & 1.6 & 1.6 & 1.7 & 1.8 \\
\hline & & orf19.288 & 1.8 & 1.5 & 3.0 & 2.5 & 2.1 & 1.5 & 2.5 & 2.0 \\
\hline \multirow[t]{7}{*}{ Biological activity unknown } & TEF4 & orf19.2652 & 16.4 & 16.6 & 55.8 & 90.2 & 29.9 & 32.7 & 65.5 & 67.8 \\
\hline & & orf19.3627 & 4.0 & 1.9 & 7.0 & 4.0 & 4.4 & 1.9 & 5.1 & 1.9 \\
\hline & $P G A 7$ & orf19.5635 & 2.3 & 3.7 & 5.7 & 7.4 & 2.7 & 3.8 & 4.8 & 6.1 \\
\hline & $B U L 1$ & orf19.5094 & 1.9 & 1.9 & 4.1 & 3.1 & 2.5 & 1.8 & 2.1 & 1.9 \\
\hline & $P G A 45$ & orf19.2451 & 1.7 & 1.5 & 2.1 & 2.2 & 1.7 & 1.9 & 2.0 & 2.3 \\
\hline & & orf19.344 & 5.1 & 6.2 & 20.2 & 16.2 & 7.2 & 6.6 & 9.6 & 9.0 \\
\hline & & orf19.5777 & 1.7 & 2.4 & 4.8 & 3.8 & 1.9 & 1.5 & 3.1 & 2.5 \\
\hline
\end{tabular}


Table 2.5. Continued.

\begin{tabular}{|c|c|c|c|c|c|c|c|c|c|c|}
\hline \multirow[b]{3}{*}{ Function $^{\mathrm{a}}$} & \multirow[b]{3}{*}{ CGD name } & \multirow[b]{3}{*}{ Orf19 designation } & \multicolumn{8}{|c|}{ Change in fold expression ${ }^{b}$} \\
\hline & & & \multicolumn{2}{|c|}{ G648S } & \multicolumn{2}{|c|}{ G648D } & \multicolumn{2}{|c|}{ Y642F } & \multicolumn{2}{|c|}{ A643V } \\
\hline & & & Exp1 & Exp2 & Exp1 & Exp2 & Exp1 & Exp2 & Exp1 & $\operatorname{Exp} 2$ \\
\hline \multirow[t]{14}{*}{ Biological activity unknown } & & orf19.5799 & 1.7 & 2.0 & 3.4 & 2.4 & 1.5 & 1.5 & 2.4 & 2.6 \\
\hline & & orf19.7456 & 1.7 & 2.6 & 4.4 & 4.1 & 2.2 & 2.1 & 3.9 & 3.5 \\
\hline & & orf19.7043 & 2.5 & 1.9 & 5.2 & 3.6 & 2.8 & 2.3 & 4.9 & 3.0 \\
\hline & & orf19.4013 & 2.6 & 2.2 & 4.2 & 3.5 & 3.1 & 2.8 & 4.8 & 3.8 \\
\hline & & orf19.4014 & 1.5 & 2.1 & 2.3 & 2.4 & 1.7 & 1.6 & 2.6 & 2.6 \\
\hline & & orf19.6840 & 2.0 & 2.1 & 4.1 & 4.4 & 2.6 & 2.4 & 3.1 & 2.6 \\
\hline & & orf19.286 & 2.1 & 2.5 & 2.3 & 2.2 & 1.9 & 1.9 & 3.5 & 1.9 \\
\hline & & orf19.7504 & 2.1 & 1.8 & 3.0 & 3.3 & 1.9 & 2.0 & 2.2 & 2.1 \\
\hline & & orf19.3737 & 1.6 & 1.9 & 2.9 & 3.4 & 2.0 & 1.8 & 3.1 & 2.6 \\
\hline & & orf19.1964 & 2.0 & 1.8 & 2.5 & 2.2 & 1.7 & 1.6 & 2.2 & 1.6 \\
\hline & & orf19.1800 & 2.3 & 2.2 & 4.6 & 3.9 & 2.5 & 2.2 & 4.5 & 3.4 \\
\hline & & orf19.7263 & 1.8 & 1.9 & 2.9 & 2.8 & 1.6 & 1.7 & 2.1 & 1.9 \\
\hline & & orf19.2496 & 1.7 & 1.8 & 2.6 & 2.5 & 1.8 & 1.7 & 2.0 & 1.5 \\
\hline & ATO9 & orf19.3261 & 1.8 & 2.2 & 2.7 & 3.4 & 1.6 & 2.3 & 4.5 & 4.5 \\
\hline
\end{tabular}

${ }^{a}$ Descriptions of gene from the Candida Genome Database (http://www.candidagenome.org).

${ }^{\mathrm{b}}$ Fold change defined as the average ratio of gene expression levels in the isolates compared in two independent microarray experiments. 


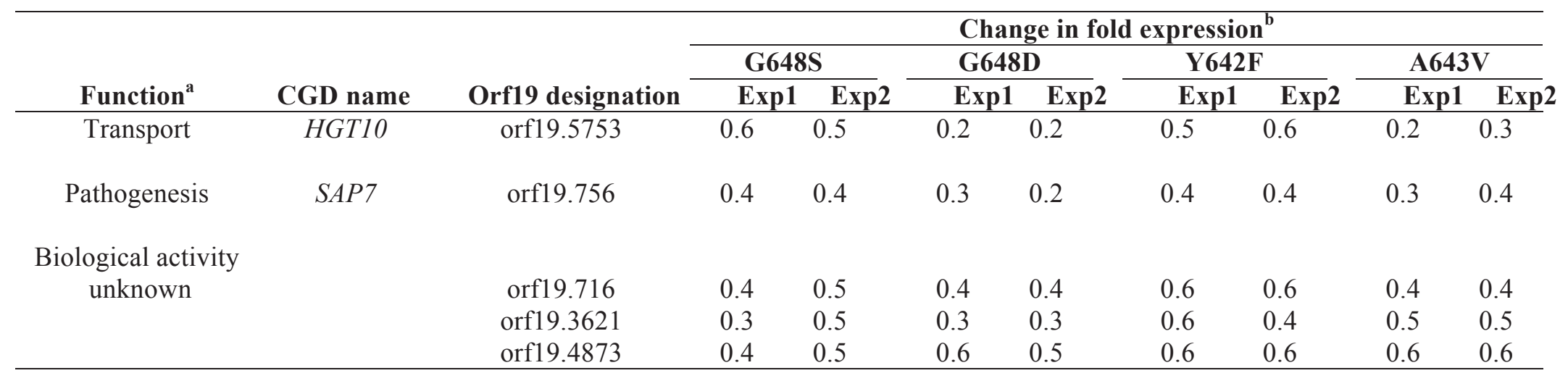

${ }^{a}$ Descriptions of gene from the Candida Genome Database (http://www.candidagenome.org).

${ }^{b}$ Fold change defined as the average ratio of gene expression levels in the isolates compared in two independent microarray experiments. 
constitutive activation, leading to the overexpression of Mdr1 $(43,127)$. Fluconazoleresistant isolates that overexpress $M D R 1$ have become homozygous for the mutated $M R R 1$ allele and work in a semidominant fashion if expressed with a wild-type allele (43). Overexpression of these transporters is known to occur among azole-resistant clinical isolates and contributes significantly to this process.

Considerably less is known about the prevalence of constitutive ERG11 overexpression and its clinical impact on azole resistance. ERG11 encodes the azole target, lanosterol demethylase, a key enzyme in the ergosterol biosynthesis pathway. $E R G 11$ is transcriptionally regulated by the zinc cluster transcription factor Upc2. In $C$. albicans, Upc2 is orthologous to two transcription factors in Saccharomyces cerevisiae, Upc2 and Ecm22, which regulate ergosterol biosynthesis and uptake of exogenous sterols $(28,34)$. The expression of these genes is affected by sterol depletion, but the exact mechanism of activation is currently unknown. Previous work in $S$. cerevisiae shows that ScUpc2 and ScEcm 22 are localized to intracellular membranes outside the nucleus, and under sterol-depleting conditions, perinuclear localization is increased (115). Gain-offunction mutations in the activation domain located near the $\mathrm{C}$ terminus have been recovered from both $\mathrm{ScUpc} 2$ and $\mathrm{ScEcm} 22$; however, mediation of ERG11 expression during hypoxic conditions or when chemically treated with azole antifungals is mediated by ScUpc2 (34).

In C. albicans, only three mutations that increase the expression of ERG11 and result in decreased susceptibility to fluconazole have been described in Upc2. In the present study, I examined a large collection of unrelated azole-resistant $C$. albicans clinical isolates for possible UPC2 GOF mutations. As fluconazole resistance in C. albicans has most often been associated with OPC, the opportunity to examine resistant isolates cultured from both oral and nonoral sources was somewhat unique. Among the isolates studied here were 16 documented nonoral isolates, 12 of which were observed to exhibit reduced susceptibilities to fluconazole. Although our analysis cannot define the clinical implications for this observation, these data underscore that fluconazole resistance extends beyond oral manifestations of candidiasis.

I further delineated the mechanisms of resistance in these isolates by determining the relative levels of expression of $C D R 1, C D R 2, M D R 1$, and $E R G 11$. Not surprisingly, $C D R 1$ and $C D R 2$ overexpression was generally coordinately regulated and quite prevalent among these isolates. Of those isolates that did overexpress $M D R 1$, even fewer isolates expressed MDR1 to the levels previously observed in azole-resistant isolates (127). ERG11 was found to be upregulated in almost three-fourths of the fluconazoleresistant isolates examined. This suggests that ERG11 overexpression is a common contributor to fluconazole resistance in C. albicans.

Among the independent azole-resistant, ERG11-overexpressing isolates studied here, I repeatedly recovered eight distinct single-nucleotide substitutions in UPC2, of which the G648S substitution occurred in nine isolates and the G648D substitution occurred in six isolates. Five of these substitutions in $U P C 2$ have not been described previously. Of the five novel mutations, four mutations resulted in increased ERG11 expression and 
increased resistance to fluconazole but to various degrees. I observed that the homozygous strain carrying the G304R UPC2 allele was more susceptible to azoles than the strain carrying the G304R substitution heterozygously; however, neither strain resulted in any decrease in antifungal susceptibility or increase in ERG11 expression. As has been previously observed in UPC2 GOF mutations, amino acid substitutions resulting from activating mutations were localized near the $\mathrm{C}$ terminus, where the activation domain of zinc-cluster transcription factors is found (109). Mutations in this region of the protein are theorized to work by one of two mechanisms: (i) mutations in this domain could relieve the transcription factor from a repressor that would otherwise keep Upc2 inactive in the absence of an activating signal or (ii) a mutation in this area could interfere with the transmembrane region of the protein, causing localization to the nucleus and constitutive activation of its target promoters.

In total, seven of the eight Upc2 mutations observed resulted in increased expression of $E R G 11$, but each mutation increased transcription to a different degree. For many strains, increased expression of ERG11 was directly correlated with the amount of ergosterol within the fungal cell, and this trend was also observed in the decreased azole susceptibility seen in strains that overexpressed ERG11. Unlike TACl GOF mutations, $U P C 2$ GOF mutations were found to occur with a wild-type allele in many isolates. I observed that strains constructed to express two mutant alleles had increased levels of ERG11 expression and decreased levels of azole susceptibility compared to the levels in strains containing both an activated Upc2 and a wild-type allele. As seen with $T A C 1$ or MRR1 mutations, a loss-of-heterozygosity event that results in two GOF alleles, either by a mitotic recombination event between homologous chromosomes or by a loss of a chromosome combined with a duplication of the homologous chromosome, would provide an advantage when challenged with antifungals $(22,127)$. Interestingly, I saw an opposite trend in the amphotericin B susceptibility of strains expressing activated UPC2 alleles, in which $U P C 2$ GOF homozygous strains with higher ERG11 expression had increased susceptibility to this agent compared to that of the heterozygous strains or the homozygous null upc $2 \Delta$ strain. This is probably due to higher levels of cellular ergosterol, which is the target of amphotericin B.

An important observation to note is the discrepancy between the levels of ERG11 expression of the clinical isolate containing a UPC2 mutation and the laboratory strain constructed to carry the same mutant $U P C 2$ allele. This difference may be due to additional levels of $E R G 11$ regulation that are developed in a clinical isolate, such as alternate transcriptional regulations or alternate single-nucleotide polymorphisms that influence ergosterol biosynthesis. Furthermore, not all ERG11 overexpression in clinical isolates was a result of a GOF mutation in $U P C 2$; therefore, it is likely that other regulators of ergosterol biosynthesis are contributing factors. Alternatively, instances of aneuploidy, specifically, chromosome 5 duplication or chr5L isochromosome formation, are known mechanisms of ERG11 overexpression in C. albicans (173). However, the $E R G 11$ expression levels I observed in many clinical isolates in this study exceed what would be expected from an extra copy of ERG11 due to the presence of a chr5L isochromosome. It is possible that some isolates that do not carry mutations in UPC2 actually overexpress $U P C 2$ instead, due to a mutation in the $U P C 2$ promoter region, gene 
duplication, or a trans-acting mutation. Whether this has occurred is being explored in ongoing investigations.

As azole resistance is mechanistically multifactorial in clinical isolates, I expected the fluconazole susceptibility of strains constructed to express specific UPC2 mutations to reflect the contribution of increased expression of genes of the ergosterol biosynthesis pathway, including ERG11, as a result of an activated UPC2 allele. In these clinical isolates, I have observed a high prevalence of mutations in the ERG11 gene itself (data not shown). It is likely that in clinical isolates, activated forms of Upc 2 occur in conjunction with mutant ERG11 alleles and that its subsequent overexpression results in a combinatorial effect on azole susceptibility. The combinatorial effects of activated UPC2 alleles and mutant ERG11 alleles on fluconazole susceptibility have not been explored.

Although microarray analysis of the G648D UPC2 GOF mutation has been performed previously, I expanded this analysis with four of the seven strongest UPC2 GOF mutations (G648D, G648S, Y642F, and A643V) for a more comprehensive examination (44). All four mutations caused the coordinate upregulation of 61 genes. As expected, many of the ergosterol biosynthesis genes (ERG1, ERG2, ERG7, ERG24, $E R G 26$, and $E R G 27$ ) were upregulated by at least 1.5 -fold over their expression in SC5314 in two separate experiments. ERG11 was upregulated in three of the four laboratory strains carrying mutations G648D, Y642F, and A643V, but elevated transcription of ERG11 could only be detected in one of the two experiments with the strain carrying the G648S allele (Appendix B). Interestingly, UPC2 was not found to be upregulated in any of the four strains analyzed by microarray analysis, although Upc 2 is thought to autoregulate and has been shown to bind its own promoter in experiments using chromatin immunoprecipitation with microarray technology (ChIP-chip) (217).

Aside from genes involved in the ergosterol biosynthesis pathway, other core sets of target genes upregulated by Upc2 included those in the gene ontology (GO) functional grouping of oxidoreductase activity. Additionally, iron ion binding was a commonly found GO category identified by the GO term finder. Not surprisingly, MDRl was overexpressed in all four strains with independent Upc2 GOF mutations; however, gene expression levels were not increased to those observed in fluconazole-resistant isolates containing an MRR1 GOF mutation. Upc2 has previously been shown to bind to the promoter of the drug efflux pump MDRl but was not a cause of Mdr1-mediated drug resistance $(169,217)$. In the present study, CDR 11 expression was increased in all independent UPC2 GOF mutations, and it increased 4.8-fold when two alleles of UPC2 carrying the predicted G648D substitution in its translated sequence were expressed. Interestingly, the gene encoding an uncharacterized $\mathrm{ABC}$ transporter, $C D R 11$, was previously shown to be upregulated by 1.5 - to 3.5 -fold in a laboratory strain carrying a single UPC2 allele containing a G648D amino acid substitution; this gene has been hypothesized to play a role in sterol transport, because of its similarity to sterol transporters in S. cerevisiae) (44).

In conclusion, I found overexpression of ERG11 to be prevalent among a collection of clinical isolates with reduced susceptibility to fluconazole and have identified and 
characterized novel $U P C 2$ gain-of-function mutations that contribute to azole resistance in clinical isolates of $C$. albicans. Overexpression of ERG11 could be explained by a gain-of-function mutation in UPC2 in many but not all cases. Gain-of-function mutations led to increased resistance to azole antifungals and terbinafine, to increased cellular ergosterol levels, and to increased expression of genes involved in ergosterol biosynthesis and oxidoreductase activity, as well as the transporter genes MDRI and CDR11. The mechanism by which $E R G 11$ is upregulated in the absence of $U P C 2$ gain-of-function mutations and the potential contribution of sterol uptake to Upc2-mediated azole resistance are under investigation. 


\section{CHAPTER 3. NDT80 MUTATIONS FOUND IN CLINICAL ISOLATES OF CANDIDA ALBICANS DO NOT CONTRIBUTE TO AZOLE RESISTANCE BY ERG11 OVEREXPRESSION}

\section{INTRODUCTION}

Not all ERG11 overexpression in azole-resistant isolates can be explained by GOF mutations in UPC2. Our lab and others have documented isolates with increased ERG11 expression that do not carry a mutated $U P C 2$ allele. These data suggest that there are alternate mechanisms of $E R G 11$ overexpression in these isolates $(50,66) . N D T 80$ is a Pho-G transcription factor that is an important in regulator of meiosis in S. cerevisiae (67). In C. albicans, NDT80 has been implicated in azole resistance not only due to its regulation of $C D R I$ but also due to its regulation of genes involved in the ergosterol biosynthesis pathway $(21,170,171,199)$. Recent ChIP-chip analysis has shown that $N D T 80$ binds to the promoter region of $E R G$ genes and also to genes involved in hyphal growth, the mitotic cycle and carbohydrate metabolism (171). A putative Ndt80 binding site has also been found in the regulatory region upstream of the UPC2 allele (73). Sellam and colleagues compared $E R G$ gene expression of a strain constructed to be deficient for NDT80 to that of wild-type and an NDT80 revertant strain (171). The mutant strain deficient for NDT80 was unable to stimulate expression of $E R G 11$ and other $E R G$ genes to the extent of either the wild-type strain or the NDT80 revertant strain in response to fluconazole. This data indicated the potential role of NDT80 in ERG gene regulation as it relates to fluconazole treatment.

In a previous search for $U P C 2$ GOF mutations, genetically matched isolate pairs 945 and 1619, and 1002 and 3795 were shown to constitutively overexpress ERG11 in the fluconazole-resistant respective counterpart (66). Sequence analysis performed on UPC2 did not detect any mutations leading to amino acid substitutions in these isolates. Due to the possible role of NDT80 in ERG gene regulation, I hypothesized that the NDT80 allele expressed in the fluconazole-resistant isolates may contain activating mutations resulting in ERG11 overexpression and azole resistance.

\section{MATERIALS AND METHODS}

\section{Strains and growth conditions}

C. albicans strains were cultured on YPD ( $1 \%$ yeast extract, $2 \%$ peptone, and $1 \%$ dextrose) agar plates at $30^{\circ} \mathrm{C}$ and stored as frozen stock in $20 \%$ glycerol at $-80^{\circ} \mathrm{C}$ (Table 3.1). YPD liquid medium was used for routine growth of strains. Nourseothricin ( 200 $\mu \mathrm{g} / \mathrm{ml}$ or $25 \mu \mathrm{g} / \mathrm{ml}$ ) was added to YPD agar plates for selection of strains containing the SAT1-flipper cassette. For plasmid construction and propagation, One Shot Escherichia coli TOP 10 chemically competent cells (Invitrogen, Carlsbad, CA) were used as a host. These strains were grown in Luria-Bertani (LB) broth or on LB agar plates supplemented 
Table 3.1. NDT80 strains and primers used in this study.

\begin{tabular}{|c|c|}
\hline $\begin{array}{c}\text { Strains and } \\
\text { primers }\end{array}$ & Relevant genotype/sequence \\
\hline SC5314 & Wild-type \\
\hline $\mathrm{ScNDT} 80^{945} \mathrm{~A}$ & NDT80 allele derived from azole-susceptible isolate \\
\hline $\mathrm{ScNDT} 80^{945} \mathrm{~B}$ & NDT80 allele derived from azole-susceptible isolate \\
\hline $\operatorname{ScNDT} 800^{1619} \mathrm{~A}$ & NDT80 allele derived from azole-resistant isolate \\
\hline $\mathrm{ScNDT} 80^{1619} \mathrm{~B}$ & NDT80 allele derived from azole-resistant isolate \\
\hline \multicolumn{2}{|l|}{ qRT-PCR } \\
\hline NDT80-F & 5'-CCT TTG GCT CAG CAA CA-3' \\
\hline NDT80-R & 5'-GCT TGG TCT TGG TTT TGT GC-3' \\
\hline$A C T 1-\mathrm{F}$ & 5'-ACGGTGAAGAAGTTGCTGCTTTAGTT-3' \\
\hline$A C T 1-\mathrm{R}$ & 5'-CGTCGTCACCGGCAAAA-3' \\
\hline$E R G 11-\mathrm{F}$ & 5'-ССССТАTТАATTTTGTTTTCCCTAATTTAC-3' \\
\hline$E R G 11-\mathrm{R}$ & 5'-CACGTTCTCTTCTCAGTTTAATTTCTTTC-3' \\
\hline \multicolumn{2}{|l|}{$\begin{array}{l}\text { NDT80 mutant } \\
\text { construction }\end{array}$} \\
\hline NDT80-A & 5'-GGG CCC GCA CCA TCA ATT TGG AGA CC-3' \\
\hline NDT80-B & 5'-ACG GCA GCT TCT TCA CTC GAG ACG TT-3' \\
\hline NDT80-C & 5'-CAC TTC ATT GGG CCC GTT CAC TAG-3' \\
\hline NDT80-D & 5'-GCC ACT AAG GTG TGA GCT CAA GAT CC-3' \\
\hline $\begin{array}{c}\text { NDT80-E } \\
\text { NDT80 } \\
\text { sequencing }\end{array}$ & 5'-CTC GAG GAT TGG TAC ACG ACA CCT GG-3' \\
\hline NDT80seqA & 5'-CAACAGAAACACCACCACCA-3' \\
\hline NDT80seq B & 5'-GCTGACGCTCAATCACAAGC-3' \\
\hline NDT80seqC & 5'-GAGTTGCCTGACCACGAGAC-3' \\
\hline NDT80seqD & 5'-ATCAACCTTTCTTCGTCATCATC-3' \\
\hline NDT80seqE & 5'-GTCCTCGTGGTCAGGCAACTC-3' \\
\hline NDT80seq F & 5'-GCTTGTGATTGAGGGTCAGC-3' \\
\hline
\end{tabular}

Underlined sequence reflects the introduction of a restriction site sequence. 
with $50 \mu \mathrm{g} / \mathrm{ml}$ kanamycin (Fisher BioReagents, Fair Lawn, NJ) or $100 \mu \mathrm{g} / \mathrm{ml}$ ampicillin (Sigma) when required.

\section{RNA isolation}

RNA was isolated using a small-scale version of the hot phenol method of RNA isolation described by Schmitt et al. Briefly, overnight cultures were diluted to an $\mathrm{OD}_{600}$ of 0.2 and then incubated at $30^{\circ} \mathrm{C}$ with shaking for an additional three or six hours to mid-log phase. Cells were collected by centrifugation, resuspended in AE buffer and then transferred to a 2-mL microcentrifuge tube containing acid phenol $(\mathrm{pH} 4.3)$ with $1 \%$ SDS. Cells were incubated at $65^{\circ} \mathrm{C}$ for 10 minutes, then lysates were clarified by centrifugation. The supernatant was then transferred into a new tube containing $900 \mu \mathrm{l}$ of chloroform and mixed. The sample was then subjected to centrifugation again, and the top aqueous layer was transferred to a new tube containing one volume of isopropanol and 0.1 volume $2 \mathrm{M}$ sodium acetate. The RNA pellet was subsequently washed with 500 $\mu 1$ of $70 \%$ ethanol and collected by centrifugation. The RNA pellet was resuspended in DNAse/RNAse-free $\mathrm{H}_{2} \mathrm{O}$. Quantity and purity were determined spectrophotometerically at absorbances of $\mathrm{A}_{260}$ and $\mathrm{A}_{280}$.

\section{Quantitative RT-PCR}

First-strand cDNAs were synthesized separately from $1 \mu \mathrm{g}$ of total RNA in a $21-\mu \mathrm{l}$ reaction volume using SuperScript first-strand synthesis system for RT-PCR (Invitrogen). Quantitative PCRs were performed in triplicate as technical replicates using the 7000 sequence detection system (Applied Biosystems). PCRs were performed, independently amplifying $18 \mathrm{~S}$ rRNA or $A C T 1$ gene (normalizing genes) and the genes of interest (GOI) from the same cDNA, using the SYBR Green PCR master mix (Applied Biosystems). Gene-specific primers were designed using Primer Express software (Applied Biosystems) synthesized by Integrated DNA Technologies (Coralville, IA) and listed in Table 3.2. PCR conditions consisted of AmpiTaq Gold activation at $95^{\circ} \mathrm{C}$ for 10 minutes, followed by 40 cycles of denaturation at $95^{\circ} \mathrm{C}$ for 15 seconds and annealing/extension at $60^{\circ} \mathrm{C}$. The dissociation curve and detection of fluorescence of SYBR Green was performed by software provided with the 7000 sequence detection system. The cycle threshold value $\left(\mathrm{C}_{\mathrm{T}}\right)$ of the normalizing gene was subtracted from the GOI to obtain the $\Delta \mathrm{C}_{\mathrm{T}}$ value. The $\Delta \mathrm{C}_{\mathrm{T}}$ value of a calibrator was subtracted of the sample $\Delta \mathrm{C}_{\mathrm{T}}$ value to obtain the $\Delta \Delta \mathrm{C}_{\mathrm{T}}$ value. Change in fold expression was obtained by calculating $2^{-\Delta \Delta \mathrm{CT}}$. Standard error was calculated from $\Delta \mathrm{C}_{\mathrm{T}}$ values as previously described.

\section{Plasmid construction for allele sequencing}

CaNDT80 coding sequences were amplified by PCR ( $P f u$ DNA polymerase; Stratagene) from C. albicans genomic DNA using the primers NDT80-A and NDT80-E (Table 3.2). Products were cloned into pCR-BLUNTII-TOPO using a Zero Blunt TOPO 
Table 3.2. NDT80 sequencing data from matched isolate sets 580, 2440 and 945, 1619 were compared to the SC5314.

\begin{tabular}{|c|c|c|}
\hline Isolate & Allele & Mutations in sequence as compared to SC5314 \\
\hline \multirow{2}{*}{$\begin{array}{l}580 \\
\mathrm{FCZ}^{\mathrm{S}}\end{array}$} & 1 & $\begin{array}{l}\text { C72T, G240A, G247A, A249G, Deletion of CAA between bp 259-261, C783T, C804T, C735G, G741A, } \\
\text { C783T, C804T, C825T, T1269C, A1398G, C1491A }\end{array}$ \\
\hline & 2 & $\begin{array}{l}\text { G243A, Deletion of CAA between bp 259-261, C783T, C804T, C735G, G741A, C783T, C804T, } \\
\text { C825T, T1269C, C1491A }\end{array}$ \\
\hline \multirow{2}{*}{$\begin{array}{l}2440 \\
\mathrm{FCZ}^{\mathrm{R}}\end{array}$} & 1 & $\begin{array}{l}\text { G243A, Deletion of CAA between bp 259-261, C783T, C804T, C735G, G741A, C783T, C804T, } \\
\text { C825T, T1269C, A1328G, C1491A }\end{array}$ \\
\hline & 2 & $\begin{array}{l}\text { G243A, Deletion of CAA between bp 259-261, C783T,C804T, C735G, G741A, C783T, C804T, C825T, } \\
\text { T1269C, C1491A }\end{array}$ \\
\hline \multirow{2}{*}{$\begin{array}{l}945 \\
\mathrm{FCZ}^{\mathrm{S}}\end{array}$} & 1 & $\begin{array}{l}\text { A240G, A246G, A249G, Addition of } 2 \text { X CAA, A393T, G430A, A486C, C741G, G747A, C810T, } \\
\text { C831T, T1276C, A1404G, C1497A }\end{array}$ \\
\hline & 2 & A240G, A246G, Addition of 1X CAA, C741G, G747A, T1276C \\
\hline \multirow{2}{*}{$\begin{array}{l}1619 \\
\mathrm{FCZ}^{\mathrm{R}}\end{array}$} & 1 & $\begin{array}{l}\text { A240G, A246G, A249G, Addition of } 2 \text { X CAA, A393T, G430A, A486C, C741G, G747A, C810T, } \\
\text { C831T, T1276C, A1404G, C1497A }\end{array}$ \\
\hline & 2 & $\begin{array}{l}\text { A240G, A246G, A249G, Addition of } 2 \text { X CAA, A393T, G430A, A486C, C741G, G747A, C810T, } \\
\text { C831T, T1276C, A1404G, C1497A }\end{array}$ \\
\hline
\end{tabular}

Bolded mutations lead to amino acid substitutions. The G340A leads to A145T a.a. substitution. The A486C mutation leads to a Q162H aa substitution. The A1328G mutations lead to a Y444C a.a. substitution. Shaded boxes defines NDT80 sequence of the second allele for each clinical isolate. 
PCR Cloning Cloning Kit (Invitrogen) and transferred into Escherichia coli TOP10 cells with selection on LB agar plates containing $50 \mu \mathrm{g} / \mathrm{ml}$ kanamycin. Plasmid DNA was purified (QIAprep; Qiagen; Germantown, MD) and sequenced on an ABI Model 3130XL Genetic Analyzer using the NDT80 sequencing primers (Table 3.2), resulting in fulllength sequence from both strands of the CaNDT80 gene. The sequencing was performed using six sets of clones derived from three independent PCRs for each strain/isolate sequenced.

Sequenced plasmids of the $E R G 11$ orf whose predicted translation indicated an amino acid substitution were digested with restriction enzymes $A p a \mathrm{I}$ and $X h o \mathrm{I}$, which excised the full-length orf from the plasmid, and the ERG11 alleles were cloned upstream of the SAT1-flipper cassette into the ApaI and XhoI sites of plasmid pSFS2. The NDT80 downstream segments were amplified with ExTaq (Takara) using primers NDT80C and NDT80D and cloned downstream of the SAT1-flipper cassette in pSFS2 using the Sac1 and $\mathrm{SacII}$ sites. This process generated plasmids pNDT80 $0^{945}$ and pNDT80 $0^{1619}$.

\section{Construction of NDT80 allele strains}

C. albicans strain SC5314 were transformed by electroporation with gel purified inserts from plasmids derived from the plasmid pSFS2. Plasmid (p)SFS2 contains the $S A T 1$-flipper disruption cassette developed by Reuss et al (156)., consisting of the SAT1 selectable marker which confers resistance to nourseothricin and the FLP flipper recombinase gene both flanked by FRT sites (flipper recombinase target sequences). Nourseothricin-resistant transformants were selected as previously described (156). Upon induction of the FLP gene, the cassette is excised such that only the NDT80 allele with a downstream FRT is left in the NDT80 locus. Integration of constructs was confirmed by Southern hybridization.

\section{Fluconazole susceptibility testing}

Minimum inhibitory concentrations (MICs) were obtained by using a modified CLSI protocol outlined in M27-A2 using RPMI. Overnight cultures grown at $30^{\circ} \mathrm{C}$ were streaked onto Sabouraud's agar. Plated cultures were grown for 24 hours at $30^{\circ} \mathrm{C}$. Individual colonies were suspended in sterile water until the optical density at $600 \mathrm{~nm}$ of 0.1 was reached. The working colony concentration was made by making a 1:50 dilution and a 1:20 dilution sequentially in media. $100 \mu 1$ from the working stock was used to inoculate a series of fluconazole/YPD media dilutions, the highest being $64 \mu \mathrm{g} / \mathrm{ml}$. Cultures were incubated at $35^{\circ} \mathrm{C}$ for 48 hours and MICs were recorded. 


\section{RESULTS}

\section{Sequencing NDT80 of matched isolate sets 945/1619 and 1002/3795 revealed multiple single nucleotide polymorphisms and a loss of heterozygosity event in the NDT80 alleles of the resistant isolate}

NDT80 in both matched isolate sets, the NDT80 allele was sequenced for both the susceptible and resistant isolates (Table 3.2). In both matched pairs, the allele expressed in the resistant isolate contained mutations that result in amino acid substitutions within Ndt80 when compared to the strain published in the Candida Genome Database (http://www.candidagenome.org/) and also when compared with the respective susceptible isolate. Additionally, sequencing of both matched sets show that in the resistant isolate, a loss of heterozygosity event had occurred so that the resistant counterpart was homozygous for one allele.

\section{Mutations observed in NDT80 of resistant isolates do not result in fluconazole resistance or increased $E R G 11$ expression}

In the fluconazole-susceptible isolate SC5314, a series of strains were constructed to test the role of mutant NDT80 alleles derived from clinical isolates on fluconazole resistance and ERG11 expression. For these experiments I used the matched isolate set 945 and 1619 as the resistant isolate 1619 had the greatest expression of ERG11 (66). Fluconazole-susceptible isolate 945 was heterozygous for NDT80, containing two different alleles of NDT80, one of which was expressed homozygously in the resistant isolate 1619. In constructed strains, each allele was expressed homozygously in isolate SC5314 (Figure 3.1). Additionally, a strain disrupted for NDT80 was also constructed for MIC comparison. When compared to SC5314, neither strain constructed to carry the NDT80 allele observed in the susceptible or the resistant isolate resulted in increased resistance to fluconazole or increased $E R G 11$ expression. I observed a two-fold decrease in fluconazole MIC in the strain in which NDT80 was disrupted (data not shown).

\section{DISCUSSION}

In C. albicans, GOF mutations in zinc-cluster transcription factors resulting in increased expression of genes involved in azole resistance are well documented. As these mutations in Tac1, Mrr1 and Upc2 all contribute to azole resistance by distinct mechanisms, $\mathrm{Ndt} 80$ has been implicated in the regulation of ABC efflux-transporters $C D R 1$ and $C D R 2$ in addition to ergosterol biosynthesis genes. Due to this global association of NDT80 with azole resistance, I hypothesized that fluconazole-resistant isolates that overexpress ERG11 independent of UPC2 GOF mutations may contain mutations in NDT80 that resulted in an azole-resistant phenotype. 
FCZ MIC

$(\mu \mathrm{g} / \mathrm{mL})$

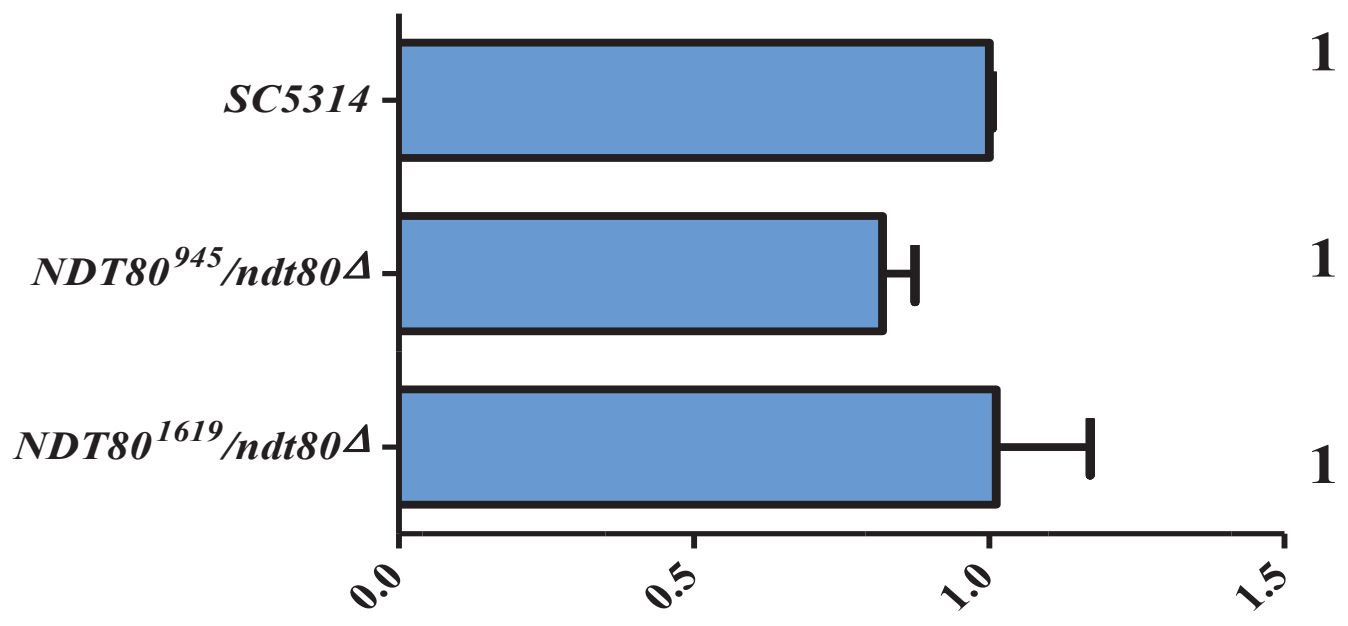

Relative Fold-Change in ERG11 Expression

Figure 3.1. ERG11 expression of strains constructed to carry NDT80 alleles derived from clinically matched isolates 945 and 1619.

ERG11 expression was measured in triplicate by qRT-PCR and fluconazole MICs were tested for strains expressing NDT80 alleles derived from fluconazole-susceptible isolate 945 or fluconazole-resistant isolate 1619. 
Previous work shows that specific mutations in the transcription factor gene $T A C 1$ regulate expression of the genes encoding $\mathrm{ABC}$ transporters $C D R 1$ and $C D R 2$. Mrr1 mediates expression of the major facilitator superfamily (MFS) transporter Mdr1 in azole-resistant isolates, and specific mutations in this transcriptional regulator result in its constitutive activation, leading to the overexpression of Mdr1. Fluconazole-resistant isolates that overexpress $M D R 1$ have become homozygous for the mutated MRRI allele and work in a semidominant fashion if expressed with a wild-type allele. Loss of heterozygosity in TACl GOF mutations has also been observed in fluconazole-resistant isolates which overexpress $C D R 1$ and $C D R 2$. In this study, matched isolate sets exhibited a similar loss of heterozygosity of NDT80 alleles in the resistant counterpart, however, neither allele resulted in overexpression of ERG11 when expressed in SC5314.

Since the completion of this study, the role of $C$. albicans NDT80 in drug resistance has been further explored. In this work, NDT80 has been shown to be dispensable to the upregulation of both $M D R 1, C D R 1$ and $C D R 2$ in response to chemical inducers benomyl and fluphenazine respectively. Additionally, this work demonstrated that disruption of NDT80 did not affect the regulation of efflux transporters or ergosterol biosynthesis genes in strains that carry GOF mutations in zinc-cluster transcription factors that mediate these processes. In other work, strains deficient for NDT80 have been shown to be deficient in biofilm formation. As the purpose of this study was to explore the role of NDT80 in ERG11 expression, strains constructed in this study have not been tested for biofilm formation or other processes. 


\section{CHAPTER 4. CLINICALLY DERIVED MUTATIONS IN ERG11 CONTRIBUTE TO AZOLE RESISTANCE IN CANDIDA ALBICANS}

\section{INTRODUCTION}

In the opportunistic fungal pathogen Candida albicans, ERG11, an important gene in the ergosterol biosynthesis pathway and target of the azole antifungals, has been shown to contribute to azole resistance in a number of ways. Overexpression of $E R G 11$, either by a gain of function mutation $(\mathrm{GOF})$ in the transcriptional regulator, $\mathrm{Upc} 2$, or increased copy number of chromosome 5, on which ERG11 resides, results in reduced azole susceptibility $(50,173)$. In addition, mutations in ERG11 that result in an amino acid substitution in lanosterol demethylase has been shown to alter the ability of the azole antifungals to bind to and inhibit this enzyme, especially in the presence of lanosterol, resulting in resistance to this class of antifungal $(85,86,200,201)$. There have been many reports of mutations in ERG11 associated with reduced azole susceptibility suggesting that lanosterol demethylase is tolerant to structural changes. Previous reports have defined three specific 'hot spot' regions that correspond to amino acids 105 to 165 , 266 to 287 , and 405 to 488 in the translated sequence that are particularly permissive to substitutions (114). In order to show that ERG11 mutations can contribute to azole resistance, investigators have used several approaches including heterologous expression of mutated ERG11 alleles in other species, enzyme inhibition with fluconazole in cell free extracts and by biochemical analysis that demonstrate these mutations contribute to azole antifungal resistance $(85,86,122,162)$. A number of different mutations have been associated with the azole-resistant phenotype but the majority of these mutations have not been studied in C. albicans background. Advances in molecular biology and our knowledge of the $C$. albicans genome now allow us to study these mutations in this pathogen itself.

In this study, I examined the prevalence and variance of ERG11 mutations in a group of 63 characterized clinical $C$. albicans isolates with reduced fluconazole susceptibility. A select group of mutant ERG11 alleles were then expressed in an azole susceptible background in order to determine the contribution of distinct mutations in ERG11 on the susceptibility of different azole antifungals.

\section{MATERIALS AND METHODS}

\section{Strains and growth conditions}

C. albicans strains were cultured on YPD (1\% yeast extract, $2 \%$ peptone, and $1 \%$ dextrose) agar plates at $30^{\circ} \mathrm{C}$ and stored as frozen stock in $20 \%$ glycerol at $-80^{\circ} \mathrm{C}$. YPD liquid medium was used for routine growth of strains. Nourseothricin $(200 \mu \mathrm{g} / \mathrm{ml}$ or 25

$\mu \mathrm{g} / \mathrm{ml}$ ) was added to YPD agar plates for selection of strains containing the $S A T 1$-flipper cassette. For plasmid construction and propagation, One Shot Escherichia coli TOP 10 
chemically competent cells (Invitrogen, Carlsbad, CA) were used as a host. These strains were grown in Luria-Bertani (LB) broth or on LB agar plates supplemented with 50 $\mu \mathrm{g} / \mathrm{ml}$ kanamycin (Fisher BioReagents, Fair Lawn, NJ) or $100 \mu \mathrm{g} / \mathrm{ml}$ ampicillin (Sigma) when required.

\section{Plasmid construction for allele sequencing}

CaERG11 coding sequences were amplified by PCR (Pfu DNA polymerase; Stratagene) from C. albicans genomic DNA using the primers ERG11-A and ERG11-E (Table 4.1). Products were cloned into pCR-BLUNTII-TOPO using a Zero Blunt TOPO PCR Cloning Kit (Invitrogen) and transferred into Escherichia coli TOP10 cells with selection on LB agar plates containing $50 \mu \mathrm{g} / \mathrm{ml}$ kanamycin. Plasmid DNA was purified (QIAprep; Qiagen; Germantown, MD) and sequenced on an ABI Model 3130XL Genetic Analyzer using the ERG11 sequencing primers (Table 4.2), resulting in full-length sequence from both strands of the CaERG11 gene. The sequencing was performed using six sets of clones derived from three independent PCRs for each strain/isolate sequenced.

Sequenced plasmids of the ERG11 orf whose predicted translation indicated an amino acid substitution were digested with restriction enzymes ApaI and XhoI, which excised the full-length orf from the plasmid, and the UPC2 alleles were cloned upstream of the SAT1-flipper cassette into the ApaI and XhoI sites of plasmid pSFS2 (156). The UPC2 downstream segments were amplified with ExTaq (Takara) using primers UPC2C and UPC2D and cloned downstream of the SAT1-flipper cassette in pSFS2 using the Not 1 and SacII sites. This process generated plasmids pERG11-2, pERG11-5, pERG11-6, pERG11-7, pERG11-7N, pERG11-8, pERG11-8O, pERG11-9, pERG11-10, pERG1113, pERG11-15/16, pERG11-19, pERG11-20, pERG11-21, pERG11-22, pERG11-27, pERG11-29 and pERG11-30.

\section{Construction of strains carrying specific ERG11 alleles}

C. albicans strain SC5314 were transformed by electroporation with gel purified inserts from plasmids (plasmids go here) derived from the plasmid pSFS2. pSFS2 contains the SAT1-flipper disruption cassette developed by Reuss et al. consisting of the $S A T 1$ selectable marker which confers resistance to nourseothricin and the FLP flipper recombinase gene both flanked by FRT sites (flipper recombinase target sequences). Nourseothricin-resistant transformants were selected as previously described.(156) Upon induction of the FLP gene, the cassette is excised such that only the ERG11 allele with a downstream FRT is left in the ERG11 locus. Integration of constructs was confirmed by Southern hybridization. 
Table 4.1. C. albicans strains carrying mutant ERG11 alleles used in this study.

\begin{tabular}{cccc}
\hline Strain & Strain background & Relevant characteristics or genotype & Source or reference \\
\hline SC5314 & N/A & $E R G 11-1 / E R G 11-2$ & ATCC \\
$\begin{array}{c}\text { Clinical Isolates } \\
10-72\end{array}$ & N/A & Azole resistant & University of Iowa
\end{tabular}

Constructed laboratory strains

\begin{tabular}{|c|c|}
\hline $5 \mathrm{~A} 2 \mathrm{~A} 43 \mathrm{~A}$ & SC5314 \\
\hline 5B6A19A & SC5314 \\
\hline 20E1II1G1 & SC5314 \\
\hline 20B5A14A & SC5314 \\
\hline 10C1B1M1 & SC5314 \\
\hline $10 \mathrm{~B} 1 \mathrm{~A} 32 \mathrm{~A}$ & SC5314 \\
\hline 2A1A18A & SC5314 \\
\hline 2B1A51A & SC5314 \\
\hline 21C1M1A1 & SC5314 \\
\hline 21B12A61B & SC5314 \\
\hline 20NA50A8A & SC5314 \\
\hline 7A5A5A & SC5314 \\
\hline 7B4A29A & SC5314 \\
\hline $15 \mathrm{~A} 3 \mathrm{~A} 108 \mathrm{~A}$ & SC5314 \\
\hline 16A14A47A & SC5314 \\
\hline 22AABA56A & SC5314 \\
\hline 22B12A $58 \mathrm{~A}$ & SC5314 \\
\hline
\end{tabular}

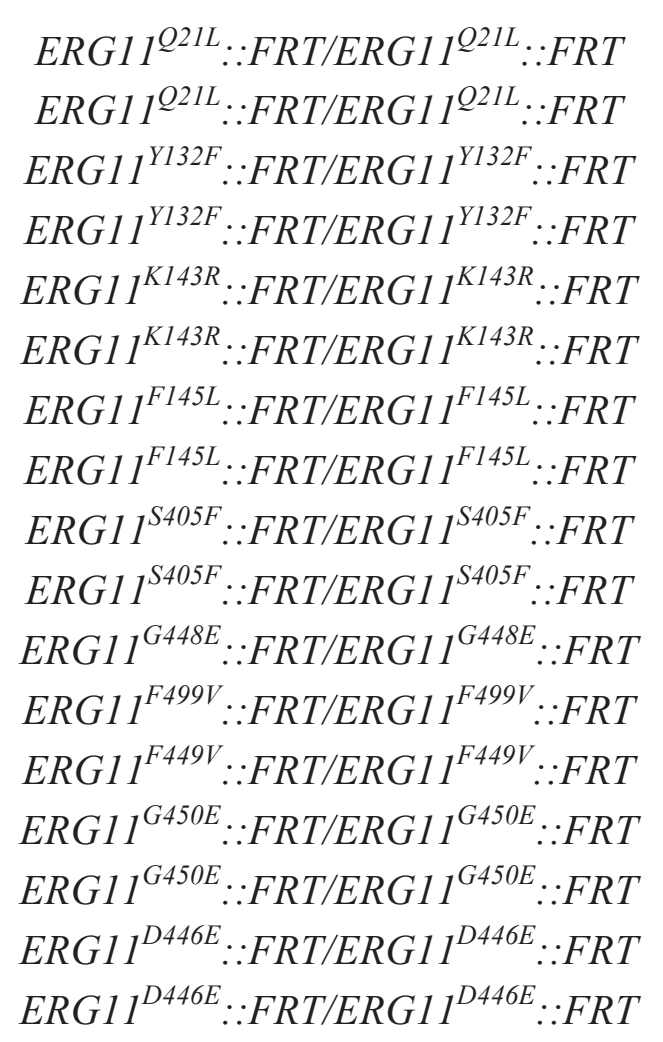

This study This study This study This study This study This study This study This study This study This study This study This study This study This study This study This study This study 
Table 4.1. Continued.

\begin{tabular}{|c|c|c|c|}
\hline Strain & Strain background & Relevant characteristics or genotype & Source or reference \\
\hline 19A1A1C1 & SC5314 & ERG11 $1^{G 464 S}: \because F R T / E R G 11^{G 464 S}: \because F R T$ & This study \\
\hline 19B1A71A & SC5314 & ERG11 $1^{G 464 S}: \because F R T / E R G 11^{G 464 S}:: F R T$ & This study \\
\hline $6 \mathrm{~A} 1 \mathrm{~A} 47 \mathrm{~A}$ & SC5314 & $E R G 11^{K 143 R, E 266 D} \because \because F R T / E R G 11^{K 143 R, E 266 D} \because \because F R T$ & This study \\
\hline $27 \mathrm{~A} 5 \mathrm{~A} 33 \mathrm{~A}$ & SC5314 & $E R G 11^{Y 132 F, F 145 L}: \because F R T / E R G 11^{Y 132 F, F 145 L} \because: F R T$ & This study \\
\hline 27B7A63A & SC5314 & ERG11 $1^{Y 132 F, F 145 L} \because F R T / E R G 11^{Y 132 F, F 145 L} \because F R T$ & This study \\
\hline 7NA35A40A & SC5314 & $E R G 11^{F 145 L, E 266 D}: \because F R T / E R G 11^{F 145 L, E 266 D}: \because \mathrm{FRT}$ & This study \\
\hline $13 \mathrm{~A} 38 \mathrm{~A} 25 \mathrm{~A}$ & SC5314 & ERG $11^{D 278 N, G 464 S} \because: F R T / E R G 11^{D 278 N, G 464 S}: \because F R T$ & This study \\
\hline 9A14A21 & SC5314 & $E R G 11^{Y 132 F, K 143 R} \because: F R T / E R G 11^{Y 132 F, K 143 R} \because: F R T$ & This study \\
\hline 9B4B34A & SC5314 & $E R G 11^{Y 132 F, K 143 R}: \because F R T / E R G 11^{Y 132 F, K 143 R}: \because F R T$ & This study \\
\hline 8OB29A48A & SC5314 & ERG11 $1^{G 307 S, G 450 E}: \because F R T / E R G 11^{G 307 S, G 450 E}: \because F R T$ & This study \\
\hline $30 \mathrm{~A} 5 \mathrm{~A} 53 \mathrm{~A}$ & SC5314 & ERG11 $1^{M 258 L, G 464 S} \because: F R T / E R G 11^{M 258 L, G 464 S}: \because F R T$ & This study \\
\hline $30 \mathrm{~B} 5 \mathrm{~A} 57 \mathrm{~A}$ & SC5314 & ERG $11^{M 258 L, G 464 S} \because: F R T / E R G 11^{M 258 L, G 464 S} \because: F R T$ & This study \\
\hline 8A4A1A & SC5314 & ERG11 $1^{I 483 V, G 450 E} \because: F R T / E R G 11^{I 483 V, G 450 E} \because: F R T$ & This study \\
\hline $8 \mathrm{~B} 4 \mathrm{~A} 47 \mathrm{~A}$ & SC5314 & ERG11 $1^{I 483 V, G 450 E} \because: F R T / E R G 11^{1483 V, G 450 E}: \because F R T$ & This study \\
\hline
\end{tabular}


Table 4.2. ERG11 primers used in this study.

\begin{tabular}{cl}
\hline Purpose, primer & Sequence \\
\hline$E R G 11$ mutant construction & \\
ERG11-A & 5'-GGGCCCGGGTTATTTGAGAACAGCC-3' \\
ERG11-B & 5'-ATCCGTTCTCGAGCACTAAGGGACAA-3' \\
ERG11-C & 5'-GTAATCAATTGAGCTCTTTTAACTTT-3' \\
ERG11-D & 5'-GATTATAGTTCCGCGGTGGTTTTACC-3' \\
ERG11-E & 5'-TGATGGTTTTTTCCACTGGCTCGAG-3' \\
& \\
$E R G 11$ sequencing & \\
T7 & 5'-TAATACGACTCACTATAGGG-3' \\
$E R G 11$ seqB & 5'-TATTTTCACTGCTTCAAGATCT-3' \\
$E R G 11$ seqC & 5'-CCAAAAGGTCATTATGTTTTAG-3' \\
$M 13 R$ & 5'-CAGGAAACAGCTATGACC-3' \\
$E R G 11$ seqE & 5'CATTTAGGTGAAAAACCTCATT-3' \\
$E R G 11$ seqF & 5'-TACTCCAGTTTTCGGTAAAGGG-3' \\
\hline
\end{tabular}

${ }^{a}$ Underlined sequence reflects the introduction of a restriction site sequence 


\section{Azole susceptibility testing}

Minimum inhibitory concentrations (MICs) were obtained by using a modified CLSI protocol outlined in M27-A2 using RPMI. Overnight cultures grown at $30^{\circ} \mathrm{C}$ were streaked onto Sabouraud's agar. Plated cultures were grown for 24 hours at $30^{\circ} \mathrm{C}$. Individual colonies were suspended in sterile water until the optical density at $600 \mathrm{~nm}$ of 0.1 was reached. The working colony concentration was made by making a 1:50 dilution and a 1:20 dilution sequentially in media. $100 \mu \mathrm{l}$ from the working stock was used to inoculate a series of azole/RPMI media dilutions, the highest being $64 \mu \mathrm{g} / \mathrm{ml}$ for fluconazole. Similar procedures were used for voriconazole and itraconazole dilutions, however the highest concentrations used for these agents was $8 \mu \mathrm{g} / \mathrm{ml}$. Cultures were incubated at $35^{\circ} \mathrm{C}$ for 48 hours and MICs were recorded.

\section{RESULTS}

\section{Many clinical isolates with reduced fluconazole susceptibility carry mutations in ERG11}

Of the 63 isolates that were determined to have a reduced susceptibility to fluconazole (MIC $\geq 8 \mu \mathrm{g} / \mathrm{mL}$ ), 55 carried a mutation in $E R G 11$ that lead to an amino acid substitution. Although silent mutations were observed in the ERG11 alleles tested (data not shown), we recovered 26 distinct positions where mutations occurred that resulted in an amino acid substitution either alone or in combination with other mutations. Each isolate was homozygous for mutations in the ERG11 allele. Nine of these substitutions, either due to their position (Q21R, M258L, L403F and I483V) or the specific amino acid substitution (A114V, D446E, Y447S, F449I and I471M) have not been described in previous reports. Among the isolates that carry amino acid substitutions in Erg11, the number of substitutions varied between the isolates and ranged between $1(n=28)$ and 4 $(\mathrm{n}=2)$. Most isolates in this collection carried a single amino acid substitution in Erg11. In total, 30 unique ERG11 alleles were recovered from sequence analysis (Table 4.3). The most common polymorphisms detected were at positions E266 ( $\mathrm{n}=10), \mathrm{Y} 132(\mathrm{n}=9)$, G464 $(n=8)$ and K143 (n=7) (Table 4.2).

\section{Single mutations in $E R G 11$ contribute to azole resistance in $C$. albicans}

In order to assess the contribution of each individual mutant ERG11 allele to azole antifungal resistance, we expressed specific mutant alleles homozygously with an identical mutant ERG11 allele (Table 4.4). Each strain was constructed in duplicate and susceptibilities were tested against fluconazole, itraconazole, and voriconazole. Azole susceptibilities of constructed strains were compared to the wild-type susceptible parent strain, SC5314 at 48 hours. 
Table 4.3. Occurrence of Erg11 amino acid substitutions in predicted translated sequence in fluconazole resistant clinical ERG11-overexpressing isolates.

\begin{tabular}{|c|c|c|c|c|c|}
\hline $\begin{array}{c}\text { A.A. substitution } \\
\text { (mutation) }\end{array}$ & ID & Zygosity & $\begin{array}{r}\text { FCZ MIC } \\
(\mu \mathrm{g} / \mathrm{mL}) \\
\end{array}$ & $\begin{array}{c}\mathrm{UPC2} \\
\text { mutation* }\end{array}$ & $\begin{array}{c}\text { Other resistance } \\
\text { mechanism* }\end{array}$ \\
\hline \multirow[t]{8}{*}{ None } & 36 & & 64 & & $\uparrow E R G 11, C D R 1, C D R 2$ \\
\hline & 37 & & 64 & & $\uparrow E R G 11, C D R 1, C D R 2$ \\
\hline & 53 & & $>256$ & & $\uparrow E R G 11$ \\
\hline & 55 & & $>256$ & G648S & $\uparrow E R G 11$ \\
\hline & 56 & & $>256$ & G648S & $\uparrow E R G 11, C D R 1, C D R 2$ \\
\hline & 57 & & $>256$ & G648S & $\uparrow E R G 11, C D R 1, C D R 2$ \\
\hline & 58 & & $>256$ & G648S & $\uparrow E R G 11, C D R 1, C D R 2$ \\
\hline & 60 & & $>256$ & & $\uparrow E R G 11$ \\
\hline Q21R (A62G) & 16 & Hererozygous & 16 & & $\uparrow M D R 1$ \\
\hline Y132F (A395T) & 48 & Homozygous & 256 & & $\uparrow E R G 11, C D R 1, C D R 2$ \\
\hline \multirow[t]{3}{*}{ K143R (A428G) } & 13 & Homozygous & 16 & A643T & $\uparrow E R G 11, C D R 1, C D R 2$ \\
\hline & 12 & Homozygous & 16 & & $\uparrow E R G 11, C D R 1, C D R 2$ \\
\hline & 14 & Homozygous & 16 & & $\uparrow E R G 11, C D R 1, C D R 2$ \\
\hline F145L (T435G) & 22 & Homozygous & 32 & & $\uparrow E R G 11$ \\
\hline F449V (T1345G) & 40 & Homozygous & 128 & & $\uparrow E R G 11, C D R 1, C D R 2$ \\
\hline
\end{tabular}


Table 4.3. Continued.

\begin{tabular}{|c|c|c|c|c|c|}
\hline $\begin{array}{c}\text { A.A. substitution } \\
\text { (mutation) }\end{array}$ & ID & Zygosity & $\begin{array}{c}\text { FCZ MIC } \\
(\mu \mathrm{g} / \mathrm{mL})\end{array}$ & $\begin{array}{c}\text { UPC2 } \\
\text { mutation* }\end{array}$ & $\begin{array}{c}\text { Other resistance } \\
\text { mechanism* }\end{array}$ \\
\hline \multirow[t]{4}{*}{ S405F (C1214T) } & 18 & Homozygous & 32 & & $\uparrow E R G 11, C D R 1, C D R 2$ \\
\hline & 19 & Homozygous & 32 & & \\
\hline & 20 & Homozygous & 32 & & $\uparrow E R G 11, C D R 1, C D R 2$ \\
\hline & 41 & Homozygous & 128 & & $\uparrow E R G 11, M D R 1$ \\
\hline \multirow[t]{3}{*}{ D446E (T1338A) } & 25 & Homozygous & 32 & G648S & $\uparrow E R G 11, M D R 1$ \\
\hline & 35 & Homozygous & 64 & G648S ${ }^{\mathrm{h}}$ & $\uparrow E R G 11$ \\
\hline & 44 & Homozygous & 128 & & $\uparrow E R G 11, C D R 1, C D R 2, M D R 1$ \\
\hline \multirow[t]{5}{*}{ G448E (G1341A) } & 31 & Homozygous & 64 & & $\uparrow E R G 11, C D R 1, C D R 2$ \\
\hline & 65 & Homozygous & $>256$ & G648S ${ }^{\text {h }}$ & $\uparrow E R G 11, C D R 1, C D R 2$ \\
\hline & 66 & Homozygous & $>256$ & & $\uparrow C D R 1, C D R 2$ \\
\hline & 67 & Homozygous & $>256$ & G648S ${ }^{\mathrm{h}}$ & $\uparrow E R G 11, C D R 1, C D R 2$ \\
\hline & 69 & Homozygous & $>256$ & G648S & $\uparrow E R G 11, C D R 1, C D R 2$ \\
\hline \multirow[t]{4}{*}{ G450E (G1349A) } & 24 & Homozygous & 32 & & $\uparrow C D R 1, C D R 2, M D R 1$ \\
\hline & 34 & Homozygous & 64 & Y642F & $\uparrow C D R 1, C D R 2, M D R 1$ \\
\hline & 63 & Homozygous & $>256$ & & $\uparrow E R G 11, C D R 1, C D R 2, M D R 1$ \\
\hline & 64 & Homozygous & $>256$ & & $\uparrow E R G 11, C D R 1, C D R 2, M D R 1$ \\
\hline \multirow[t]{2}{*}{ G464S (G1390A) } & 32 & Homozygous & 64 & & $\uparrow C D R 1, C D R 2, M D R 1$ \\
\hline & 38 & Homozygous & 64 & G648D ${ }^{\mathrm{h}}$ & $\uparrow E R G 11, C D R 1, C D R 2$ \\
\hline
\end{tabular}


Table 4.3. Continued.

\begin{tabular}{|c|c|c|c|c|c|}
\hline $\begin{array}{l}\text { A.A. substitution } \\
\text { (mutation) }\end{array}$ & ID & Zygosity & $\begin{array}{c}\text { FCZ MIC } \\
(\mu \mathrm{g} / \mathrm{mL})\end{array}$ & $\begin{array}{c}\text { UPC2 } \\
\text { mutation* }\end{array}$ & $\begin{array}{l}\text { Other resistance } \\
\text { mechanism* }\end{array}$ \\
\hline G464S (G1390A) & 39 & Homozygous & 64 & & $\uparrow E R G 11, C D R 1, C D R 2$ \\
\hline continued & 42 & Homozygous & 128 & & $\uparrow E R G 11, C D R 1, C D R 2$ \\
\hline A114S (G340T), Y257H (T769C) & 17 & Homozygous & 32 & & $\uparrow C D R 1, C D R 2$ \\
\hline F126L (C378A), Y132F (A395T) & 30 & Homozygous & 64 & $\mathrm{~A} 643 \mathrm{~V}^{\mathrm{h}}$ & $\uparrow E R G 11$ \\
\hline \multirow[t]{2}{*}{ Y132F (A395T), K143R (A428G) } & 50 & Homozygous & 256 & & $\uparrow C D R 1, C D R 2$ \\
\hline & 61 & Homozygous & $>256$ & & $\uparrow C D R 1, C D R 2$ \\
\hline Y132F (A395T), F145L (T435G) & 29 & Homozygous & 64 & & $\uparrow C D R 1, C D R 2$ \\
\hline K143R (A428G), E266D (A798C) & 10 & Homozygous & 16 & & $\uparrow E R G 11, C D R 1$ \\
\hline F145L (T435G), E266D (A798C) & 33 & Homozygous & 64 & Y642F & $\uparrow C D R 1, C D R 2$ \\
\hline $\begin{array}{c}\text { M258L (A772T), G464S } \\
\text { (G1390A) }\end{array}$ & 70 & Homozygous & $>256$ & $\mathrm{~A} 646 \mathrm{~V}^{\mathrm{h}}$ & $\uparrow E R G 11$ \\
\hline \multirow[t]{3}{*}{$\begin{array}{c}\text { E266D (A798C), G464S } \\
\text { (G1390A) }\end{array}$} & 45 & Homozygous & 128 & $\mathrm{~A} 646 \mathrm{~V}^{\mathrm{h}}$ & $\uparrow E R G 11 . C D R 1 . C D R 2$ \\
\hline & 15 & Homozygous & 16 & W478Ch & $\uparrow E R G 11, C D R 1, C D R 2$ \\
\hline & 27 & Homozygous & 64 & W478C & $\uparrow E R G 11, C D R 1, C D R 2$ \\
\hline
\end{tabular}


Table 4.3. Continued.

\begin{tabular}{|c|c|c|c|c|c|}
\hline $\begin{array}{l}\text { A.A. substitution } \\
\text { (mutation) }\end{array}$ & ID & Zygosity & $\begin{array}{c}\text { FCZ MIC } \\
(\mu \mathrm{g} / \mathrm{mL})\end{array}$ & $\begin{array}{c}\text { UPC2 } \\
\text { mutation* }\end{array}$ & $\begin{array}{l}\text { Other resistance } \\
\text { mechanism* }\end{array}$ \\
\hline \multicolumn{6}{|l|}{ D278N (G832A), G464S } \\
\hline$(\mathrm{G} 1390 \mathrm{~A})$ & 43 & Homozygous & 128 & & $\uparrow C D R 1, C D R 2$ \\
\hline & 51 & Homozygous & 256 & & $\uparrow E R G 11, C D R 1, C D R 2$ \\
\hline & 62 & Homozygous & $>256$ & & $\uparrow C D R 1, C D R 2$ \\
\hline \multirow[t]{2}{*}{ G307S (G919A), G448R } & 26 & Homozygous & 32 & & \\
\hline & 54 & Homozygous & $>256$ & & $\uparrow E R G 11, M D R 1$ \\
\hline $\begin{array}{c}\text { G450E }(\mathrm{G} 1349 \mathrm{~A}), \mathrm{I} 483 \mathrm{~V} \\
\text { (A1309G) }\end{array}$ & 23 & Homozygous & 32 & & $\uparrow C D R 1, C D R 2, M D R 1$ \\
\hline $\begin{array}{c}\text { A114V (C341T), E226D (A798C), } \\
\text { H283R (A848G) }\end{array}$ & 21 & Homozygous & 32 & G648D ${ }^{h}$ & $\uparrow E R G 11, C D R 1, C D R 2$ \\
\hline $\begin{array}{c}\text { Y132F (A395T), T229A (A685G), } \\
\text { F449L (T1345A) }\end{array}$ & 59 & Homozygous & $>256$ & G648D ${ }^{\mathrm{h}}$ & $\uparrow E R G 11, C D R 1, C D R 2$ \\
\hline $\begin{array}{c}\text { Y132F (A395T), V437I (G1309A) } \\
\text {, F449L (T1345C) }\end{array}$ & 71 & Homozygous & $>256$ & G648D ${ }^{\mathrm{h}}$ & $\uparrow E R G 11, C D R 1, C D R 2$ \\
\hline $\begin{array}{c}\text { G307S (G919A), L403F } \\
\text { (A1209T), G448R(G1342C) }\end{array}$ & 46 & Homozygous & 128 & & \\
\hline $\begin{array}{c}\text { G307S (G919A), V437I } \\
\text { (G1309A), Y447S (A1340C) }\end{array}$ & 49 & Homozygous & 256 & & $\uparrow E R G 11, C D R 1, C D R 2$ \\
\hline
\end{tabular}


Table 4.3. Continued.

\begin{tabular}{|c|c|c|c|c|c|}
\hline $\begin{array}{c}\text { A.A. substitution } \\
\text { (mutation) }\end{array}$ & ID & Zygosity & $\begin{array}{c}\text { FCZ MIC } \\
(\mu \mathrm{g} / \mathrm{mL})\end{array}$ & $\begin{array}{c}\text { UPC2 } \\
\text { mutation* }\end{array}$ & $\begin{array}{l}\text { Other resistance } \\
\text { mechanism* }\end{array}$ \\
\hline $\begin{array}{c}\text { A114V (C341T), D153E (T459G) } \\
\text {, E266D (A798C), G450E } \\
(\text { G1349A) }\end{array}$ & 72 & Homozygous & $>256$ & & $\uparrow C D R 1, C D R 2$ \\
\hline $\begin{array}{c}\text { A114V (C341T), Y132F (A395T), } \\
\text { E266D (A798C), V437I } \\
(\mathrm{G} 1309 \mathrm{~A})\end{array}$ & 47 & Homozygous & 128 & G648D ${ }^{\text {h }}$ & $\uparrow E R G 11, C D R 1, C D R 2$ \\
\hline $\begin{array}{c}\text { A114V (C341T), Y132F (A395T), } \\
\text { E266D (A798C), V437I } \\
\text { (G1309A) }\end{array}$ & 47 & Homozygous & 128 & G648D ${ }^{\mathrm{h}}$ & $\uparrow E R G 11, C D R 1, C D R 2$ \\
\hline
\end{tabular}

*Data previously published in Flowers, S. A., K. S. Barker, E. L. Berkow, G. Toner, S. G. Chadwick, S. E. Gygax, J. Morschhauser, and P. D. Rogers. 2012. Gain-of-function mutations in UPC2 are a frequent cause of ERG11 upregulation in azole-resistant clinical isolates of Candida albicans. Eukaryot Cell 11: 1289-99 (50). ${ }^{\mathrm{h}}$ Amino acid substitution recovered in one of two UPC2 alleles. 
I initially examined the effects of mutant $E R G 11$ alleles containing a single amino acid substitution in the predicted protein sequence (Table 4.4). I identified 10 distinct ERG11 alleles containing a single amino acid substitution (Q21L, Y132F, K134R, F145L, F449V, S405F, D446E, G448E, G450E and G464S). With the exception of amino acid substitutions Q21L and G448E, most ERG11 mutations resulted in decreased susceptibility to fluconazole when compared to SC5314. Strains homozygously expressing K143R resulted in the strongest decrease in fluconazole susceptibility. Strains that carried two alleles containing amino acid substitutions Y132F, F145L, S405F, D446E, F449V G464S, and G450E had at least a four-fold increase in fluconazole MICs. No single mutation significantly affected itraconazole or voriconazole susceptibilities. These data suggest that structural differences between specific azoles affect activity against specific mutant $E R G 11$ alleles.

\section{Multiple mutations in ERG11 can result in decreased azole susceptibility}

Previous observations show that combinations of ERG11 mutations can lead to considerable increases in MICs to fluconazole. To examine the effects of multiple mutations on azole susceptibility, I selected a group of clinically occurring ERG11 alleles that carried two amino acid substitutions. I were specifically interested in evaluating azole-susceptibilities of strains containing two amino acid substitutions, in which at least one substitution has been characterized alone (see above).

The K143R + Y132F was the strongest characterized combination resulting in increased FCZ MICs and VCZ MICs of ten-fold and four-fold respectively over what was observed for SC5314. Interestingly, this combination did not affect ICZ susceptibilities. Other notable increases in FCZ MICs where detected when characterizing the $\mathrm{Y} 132 \mathrm{~F}+\mathrm{F} 145 \mathrm{~L}$ and the G307S + G450E combinations, both resulting in an increase in FCZ MIC of eight-fold over that of SC5314. Notably, the Y132F + F145L combination also demonstrated significant effects on both ICZ and VCZ with an increase of MICs by four-fold and eight-fold respectively. Notably, this was the only mutant $E R G 11$ allele characterized in this study that produced a significant decrease in ICZ susceptibility.

I characterized other combinations of polymorphisms that arose clinically in our collection. I483V + G450E in combination increased FCZ MICs six-fold over SC5314 and two-fold over what was observed for G450E as a single polymrphism. This combination did not result significant changes to either ICZ or VCZ MICs. I observed a similar trend in investigating the effects of the addition of M258L when combined with G464S, in which the combination increased FCZ MICs by two-fold over G464S alone. 
Table 4.4. Forty-eight hour MICs to FCZ, ICZ and VCZ measured by using a modified CLSI broth microdilution methods of $C$. albicans constructed to carry mutant $E R G 11$ alleles homozygously.

\begin{tabular}{cccc}
\hline & & $\mathbf{4 8} \mathbf{h o u r} \mathbf{M I C}(\boldsymbol{\mu g} / \mathbf{m L})$ & \\
\cline { 2 - 4 } Erg11 SNP & FCZ $^{*}$ & $\mathbf{I C Z}$ & $\mathbf{V Z Z}^{*}$ \\
\hline SC5314 & 0.25 & 0.016 & 0.016 \\
Q21L & 0.5 & 0.016 & 0.016 \\
Y132F & 2 & 0.016 & 0.03 \\
K143R & 4 & 0.06 & 0.03 \\
F145L & 1 & 0.03 & 0.016 \\
S405F & 2 & 0.03 & 0.016 \\
D446E & 1 & 0.016 & 0.016 \\
F449V & $1-2$ & 0.03 & 0.016 \\
G448E & 0.5 & 0.03 & 0.016 \\
G450E & 1 & 0.03 & 0.016 \\
G464S & 1 & 0.03 & 0.016 \\
K143R, E226D & 4 & 0.016 & 0.016 \\
Y132F, F145L & $4-8$ & 0.06 & 0.25 \\
I483V, G450E & 2 & 0.03 & $0.016-0.03$ \\
Y132F, K143R & 8 & 0.03 & 0.06 \\
F145L, E266D & 1 & 0.03 & 0.016 \\
D278N, G464S & 4 & 0.016 & 0.016 \\
E266D, G464S & 1 & 0.016 & 0.016 \\
M258L, G464S & 2 & 0.016 & 0.03 \\
G307S, G450E & 4 & 0.016 & 0.016 \\
\hline
\end{tabular}

*FCZ, fluconazole; ICZ, itraconazole; VCZ, voriconazole 


\section{Amino acid substitution E266D does not contribute to azole resistance}

E266D was the most prevalent polymorphism detected by sequence analysis and occurred only in combination with other amino acid substitutions. To investigate the contribution of E266D to azole resistance, I compared the susceptibilities of ERG11 alleles carrying one amino acid substitution alone to an allele containing an identical amino acid substitution combined with E266D. In our collection, K143R, F145L and G464S all clinically occurred as a single mutation and occurred combined with E266D in different isolates. In all three instances, I did not observe E266D to confer any additional effect on azole susceptibility beyond what was observed with the original amino acid substitution.

\section{DISCUSSION}

Resistance to azole antifungals in C. albicans is attributable to a number of distinct of mechanisms. It is well established that activating mutations in transcription factors regulating genes encoding efflux pumps mediate resistance to azole antifungals. Previous work has shown specific mutations in the transcription factor gene TAC1 mediate expression of $\mathrm{ABC}$ transporters $C D R 1$ and $C D R 2$, resulting in increased azole resistancein C. albicans (22). Similarly, Mrr1 has been identified as the regulator of the major facilitator superfamily (MFS) transporter Mdr1 in azole-resistant isolates, and GOF mutations in this transcriptional regulator produce constitutive activation (43). I have previously characterized the expression of genes involved in azole resistance in this large group of clinical isolates with decreased fluconazole susceptibility (50). I identified several mutations in the transcription factor $U P C 2$, which resulted in the constitutive activation genes involved in the ergosterol biosynthesis pathway, including ERG11. In this study, I determined the frequency, variability and the specific contributions of ERG11 mutations from this characterized group of clinical isolates to azole resistance.

The cytochrome P450 (CYP) superfamily of enzymes contains more than 2500 members that can be roughly placed into two groups stratified by their function (http://drnelson.uthsc.edu) (98). In the first group, members metabolize a wide variety of xenobiotics while those in the second group generally participate in key biosynthetic processes such as sterol biosynthesis. Substrate specificity for this second group of P450s is narrow. Sterol 14 $\alpha$-demethylase (Cyp51 or Erg11) is considered to be the most ancient CYP family because it is the only P450 class that is found in different kingdoms such as animals, plants and bacteria $(130,210)$. In 2009, the first observed virally encoded CYP450 gene was observed and exhibited low-level sequence homology to CYP51 genes although its function remains unknown (95). Despite only the $22-33 \%$ of sequence homology demonstrated between kingdoms, there are only four known substrates for this class of enzymes. This substrate preservation between kingdoms suggests that the Cyp51 structure is highly conserved regardless of low sequence homology (Figure 4.1). There are 41 amino acids conserved between the animal, bacterial and fungal Cyp51 enzymes (98). Of those 41 amino acids, there are three positions that are conserved in all CYP450 
Figure 4.1. Cyp51 secondary structure is conserved despite low sequence homology between kingdoms.

Alignment of C. albicans, T. cruzi, M. tuberculosis, and Wheat Cyp51 proteins performed by UniProt www.uniprot.org. Pink highlights alpha helical structure and purple highlights beta sheets as predicted by crystal structure when available. Light blue highlighted heme coordinating cysteine is conserved in all CYP450 enzymes. *indicates conserved amino acid residues in all represented organisms. The following accession numbers were used: C. albicans-P10613; T. cruzi-Q7Z1V1; T. aestivum-P93596; $M$. tuberculosis-P0A512. 
MAIVETVIDGINYFLSLSVTOOISILLGVP FVNLVUT-OYLYSLRKDRAP L VFYTIPUFG ----------MFI-----EAIVLALTALILYSVYSVKS FNTTRPTD P PVYPVTVPFLG

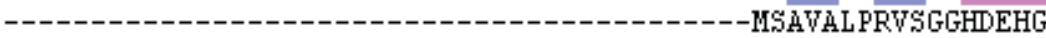
$+$

SAASYGQQPYE FFESCRQKY-GDVFS FMLLGKIMTVYLGPKGHE FVFNAKLSDVSAEDAY HIVQ FGKNI LE FMORCKRD LKS GV FTISI GGQRVTIV GD PHE HSR FFSPRNE I LSPREVY HLEE FRTDP I GLMQRVRDEC-GDVGTFQLAGKQVWLLSGSHAMNEFFFRAGDDDLDQAKAYY GLLRFLRGPIPLIRAEYARL-GPVFTVPILTRRITFLIGPDVSAHFFKSNESDMSQQEVY

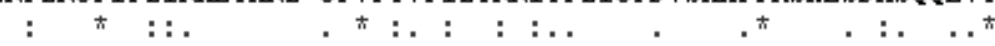

406

384

349

KHLTTPVFGKGVIYDCPNSRLMEQKKFAKFALTTD SFKRYVPKIREEILNYFVTDES FKL T-

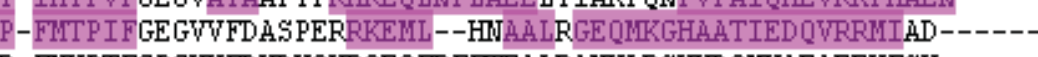
R-FWVPTFGPGWFDVDYQVRQEQFRFFTEALRANKLRSYVDQNWAEAEEYF : . औ $\quad$ : : : . : : . :

KEK-THGVANVMKTQPEITIFTASRSLFGDEMRRIFD-RSFAQLYSDLDKGFTPINFVFP

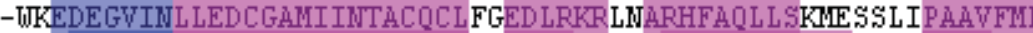
-UG-EAGE ID LLDFFÅE LTIYTSSACLIGKKFRDQLD-GRFAKLYHE LERGTDPLAYVDP -WG-ESGTVD LKYELEHLIILTASSRCLLGREVREKLF-DDVSALFHDLDNGMLP ISVIFP

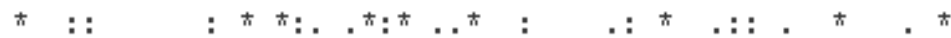

N---L P L PHYTRRDAДQKKI SATYMKE IKSRR-ERGD ID PNRD L IDSL L IHSTYKDGVKWLLRL PLPQSARCREARAE LQKILGEI IVAREKEEASKDNNTSDL LGGLLKAVYRDGTRY---LP IES FRRRDEARRNGL VALVAD IMINGRI-ANNP PTDKSDRDMLDVLIAYKÄETGTPF Y---LPIPAHRRRDQARRTRLAEIFATIIKSRK-ÄS---GQSEEDMLQCFIDSKYKNGRQ: . *

MTDQE IANL LIGILMGGQHTSASTSAJFLLHLGEKPH--LQDVIYQEVVELLKEKGGDLN

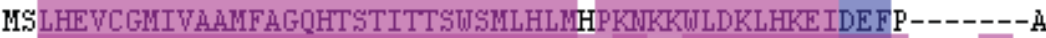
FSADE ITGMFISMFÄGHHTSSGTẢSTTL IE LMRHRD--AYYA ÂV IDELDE LY----GDGR TTESEVTGLLIAALFAGQHTSSITSTWTGAYLLKFQQ--YFAEAVEEQKEVMK---RHGD

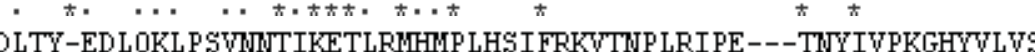
QLNYDNVIDEMP FAERCVRES IRRD P P LLMVMRMVRAE VKV GS-----YWVPKGDIIACS SVSF-HALRQIPQLENVLKETLRLHP P LIILMRVAKGE FEVQG-----HRIHE GDLVA ÀA KIDH-DILAEMDVLYRCIKEALRLHP PLIMLLRQSHSDFSVTTREGKE FD IPKGHIVAT

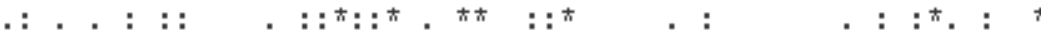

PGYAHTSERYFDNPED FD PTRUDTAДAKANSVSFNSSDEVDYGFGKVSKGVSSPYLPFGG

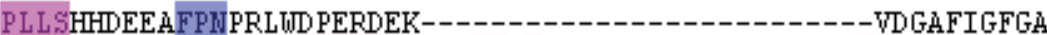
PAISNRI PED FPD PHD FVP ARYEQPRQED-L---------------LNRWTWIPFGA PAFANRL PHIFKNPDSYD PDRFALGREEDKV--------------A ÄGAFSYISFGG * : . . $:^{*}:$ * $^{*}$ :

GRHRCIGEQFAYWQLGTILTTFVNLRUTIDG-YKVPDPDYSSNWV PTEPAEIIWEKRE GVHKCIGQKFA L LQVKTILÅTẢFREYD FQLLR-DEVPD PDYHTWVVGPTLNQCLVKYTRK GRHRCVGÅFA IMOIKAIF SVL LREYE FEMAQP PE SYRNDH SKMVVQLAOPACVRYRRRT GRHGCLGEPFAYLQIKAIITHL LRNFE FELVSP FP--ENDTNAMWVGIKGEVNVNYKRRK

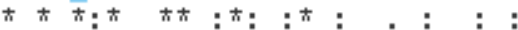

TCMF- 528

KKLPS 481

GV--- 451

LIVDN 453

C. albicans

T. cruzi

M. tuberculosis

Wheat
C. albicans

T. cruzi

M. tuberculosis

Wheat

C. albicans

T. cruzi

M. tuberculosis

Wheat

C. albicans

T. cruzi

M. tuberculosis

Wheat

C. albicans

T. cruzi

M. tuberculosis

Wheat

C. albicans

T. cruzi

M. tuberculosis

Wheat

C. albicans

T. cruzi

M. tuberculosis

Wheat

C. albicans

T. cruzi

M. tuberculosis

Wheat

C. albicans

T. cruzi

M. tuberculosis

Wheat

C. albicans

T. cruzi

M. tuberculosis Wheat 
enzymes. One of these positions is a cysteine thiolate that coordinates the heme group while the other two are the ExxR salt bridge in the K-helix (98).

Sterols are an essential component of the eukaryotic membrane that functions to maintain fluidity as well as other regulatory processes. The azole class of antifungals function not only to inhibit ergosterol biosynthesis but also result in the accumulation of toxic methylated sterol precursors (24). The primary sterol in the fungal cell membrane is ergosterol and CYP51 in C. albicans is the rate limiting step in this biosynthetic process.

CaCyp51 catalyzes a three-step reaction that ultimately results in the demethylation of lanosterol. Each step requires one molecule of oxygen and NADPH. The azoles inhibit lanosterol demethylase by binding of the nucleophilic $\mathrm{N}_{4}$ atom of the azole ring to the heme iron at its sixth coordinate position (162). The normal substrate for this enzyme is lanosterol and azole derivatives sit in the same binding pocket. Biochemical analysis show all azoles bind selectively to CaCyp51; however, Kd values show a two-to four-fold lower affinity to fluconazole when compared to itraconazole or voriconazole (200).

In agreement with previous studies, the majority of our clinical isolates carried point mutations in ERG11 that lead to amino acid substitutions (122). In our collection, $87 \%$ of all isolates carried at least one nonsynonomous mutation that resulted in an amino acid substitution. Previous work has also defined mutations that occur in ERG11 susceptible isolates which means the presence of an ERG11 mutation may not be adequate to predict azole susceptibility. Substitutions in our collection were recovered in 26 distinct positions in which all but five were located in previously defined "hot spot" regions where CaERG11 mutations frequently occur. In our constructed strains, most ERG11 alleles containing single nucleotide changes resulted in meaningful changes in fluconazole MICs but no single mutation conferred significant changes in either itraconazole or voriconazole susceptibilities. K143R produced the strongest increase in fluconazole MICs in this study as a single amino acid substitution. Y132F also conferred increased resistance to fluconazole. Previous analysis performed with heterologous expression of CaCyp51(Y132H) in S. cerevisiae revealed a spectral change indicative of the inability of the azole nitrogen to interact in a typical fashion with the Cyp51 heme (85). Both the K143 and Y123 positions are conserved among fungal species and have been associated with resistance in previous work $(5,162)$.

Sanglard et al. have formally determined that Cyp51 amino acid substitutions S405F, $\mathrm{Y} 132 \mathrm{H}$, and G464S independently resulted in increased azole resistance when heterologously expressed in S. cerevisiae (162). The S405 position is a highly conserved residue of the Cyp51 in different organisms and this mutation is exclusively recovered from azole-resistant strains (122). In our collection, $\mathrm{S} 405 \mathrm{~F}$ was recovered in four distinct clinical isolates and was only recovered as a single polymorphism. In another conserved position, the G464S amino acid substitution also resulted in increased fluconazole resistance in strains carrying two mutant ERG11 alleles. Previous spectral analysis of the G464S mutation reveals that this modification exerts its effects by a change in the heme environment(86).In this work, Kelly et al demonstrated the G464S substitution reduced affinity for fluconazole but the enzyme retained its catalytic activity. 
In a fungal specific major insertion loop, I recovered multiple amino acid substitutions that occurred as single mutations and also combined with other amino acid substitutions. Modeling of amino acid residues at these positions is difficult due to the lack of fungal crystal structures. Homology modeling of this insertion loop was investigated previously in $P$. pastoris (5). Among fungal species, this unique sequence length varies and in C. albicans, this loop is comprised of 31 amino acid residues (5). Sequence analysis done for this loop in different fungal species show that the N-terminal portion of is variable in sequence and length while the $\mathrm{C}$-terminal portion contains acidic residues followed by a more invariable portion with the motif DYG[FY]Gx[VI][ST]KG (Figure 4.2) (5). In this collection, amino acid substitutions were recovered at positions D446, G448, F449, and G450. These positions all occur within the C-terminal invariable motif within the fungal specific major insertion loop (Figure 4.3). Interestingly, mutations that occur independently at these position all increase fluconazole MICs by about fourfold in strains that express homozygous mutant ERG11 alleles. No amino acid substitution at these positions either occurring as a single mutation, or when combined with another amino acid substitution affects susceptibilities to either itraconazole or voriconazole.

I also examined the collective effects of multiple mutations in ERG11 that were derived from clinical isolates. Specifically, I was interested in characterizing the effects of amino acid substitutions that occurred with a mutation that had been characterized alone. The K143R + Y132F combination resulted in the strongest increase in FCZ MIC by increasing fluconazole and voriconazole MICs by ten-fold and four-fold respectively over what was observed for SC5314. This combination occurred independently in two isolates that were highly resistant to fluconazole (MIC $\geq 256 \mu \mathrm{g} / \mathrm{mL}$ ) accompanied with increased expression of $\mathrm{ABC}$ transporters $C D R 1$ and $C D R 2$ in both isolates. F145L + Y132F were the only amino acid substitutions that significantly affected MICs to all azoles. Previous modeling in Aspergillus fumigatis defined F145 as a position that interacts with posaconazole (208). Structurally, itraconazole is similar to posaconazole and notably, this combination was the only combination of amino acid substitutions that significantly affected susceptibility to itraconazole. This combination of substitutions was also shown to catalytically impair Cyp51 when derived from a clinical C. albicans isolate. Investigation of Cyp51 function in Aspergillus fumigatis showed that positions G464S and G307 disturb the heme environment (208). Our observed susceptibility phenotypes show increased fluconazole MICs to G464S and the combination of G307S + G450E. The combination of G307S + G450E increased fluconazole MICs beyond what was observed with G450E as a single mutation.

Amino acid substitutions I483V, M258L, D278N, were demonstrated to have additive effects on fluconazole resistance when combined with an amino acid substitution that has been characterized alone. Substitution I483V has not been described previously in the literature and does not seem to be located near the active site when considering CaCyp51 secondary structure. When combined with substitution G450E, fluconazole MICs increase by two-fold over that which was observed for G450E alone. A similar trend was 


\begin{tabular}{|c|c|c|c|}
\hline 404 & VSPGYAHTSERYFDNPED FDPTRUDTAДAKANSVSFN-----SSDEVDYGFGKVSKGVS & 458 & C. albicans \\
\hline 408 & VSPGYTHLRDEYFPNÄHQFNIHRURKDSASSYS---------VGEEVDYGFGAISKGVSS & 458 & S. cerevisiae \\
\hline 398 & SSPGVTARDERHFRD P LRUD PHRUESRVEV----------EDSSDTVDYGYGAVSKGTRS & 447 & P. italicum \\
\hline 404 & VSAGYAHTSDRUFEHPEHFNPRRUE SDDTKÄSAVSFN-----SEDTVDYGFGKISKGVSS & 458 & C. tropicalis \\
\hline & ÄAPGLTÄTEEEYFTHÄTD FD PKRUTNDRVNE----------DENAEQIDYGYGLVTKGÄÁS & 430 & S. pombe \\
\hline & ISPGYTML SERYFPNASE FQPHRUDE IKS IDGGISFGAEGENAKETVDYGFGKISKGVAS & 401 & C. krusei \\
\hline & ASPAISNRI PED FPD PHD FVP ARYEQ PRQEDL L $-\cdots-\cdots-\cdots$ & 382 & M. tuberculosis \\
\hline
\end{tabular}

Figure 4.2. Sequence alignment highlighting the fungal specific major insertion loop in various fungal species and contrasted with the non-fungal species, M. tuberculosis.

Alignment was generated by UniProt www.uniprot.org. The red bracketed sequence defines the invariable sequence where we have observed amino acid modifications which affect fluconazole susceptibility. The following accession numbers were used: $C$. albicansP10613; C. tropicalis-P14263; S. cerevisiae-P10614; P. italicum-Q12664; S. pombe-Q09736; C. glabrata-P50859; C. krusei-Q02315; M. tuberculosis-P0A512. 
Figure 4.3. Observed amino acid subsitutions in Erg11 in C. albicans compared to other medically important Candida species and organisms in other kingdoms.

The alignment was generated by using the UniProt alignment function www.uniprot.org. Amino acid numbering is based off the $C$. albicans sequence. Conserved amino acids are highlighted in blue. Amino acid substitutions highlighted in red are those observed in this study. Substitutions observed in black were noted in the literature. The following accession numbers were used: $C$. albicans-P1613; C. tropicalis-P14263; S. cerevisiae-P1-614; C. glabrata-P50859; C. krusei-Q02315; Human-Q16850; M. tuberculosis-POA512; T. Aestivum-P93596. 
Amino Acid Position

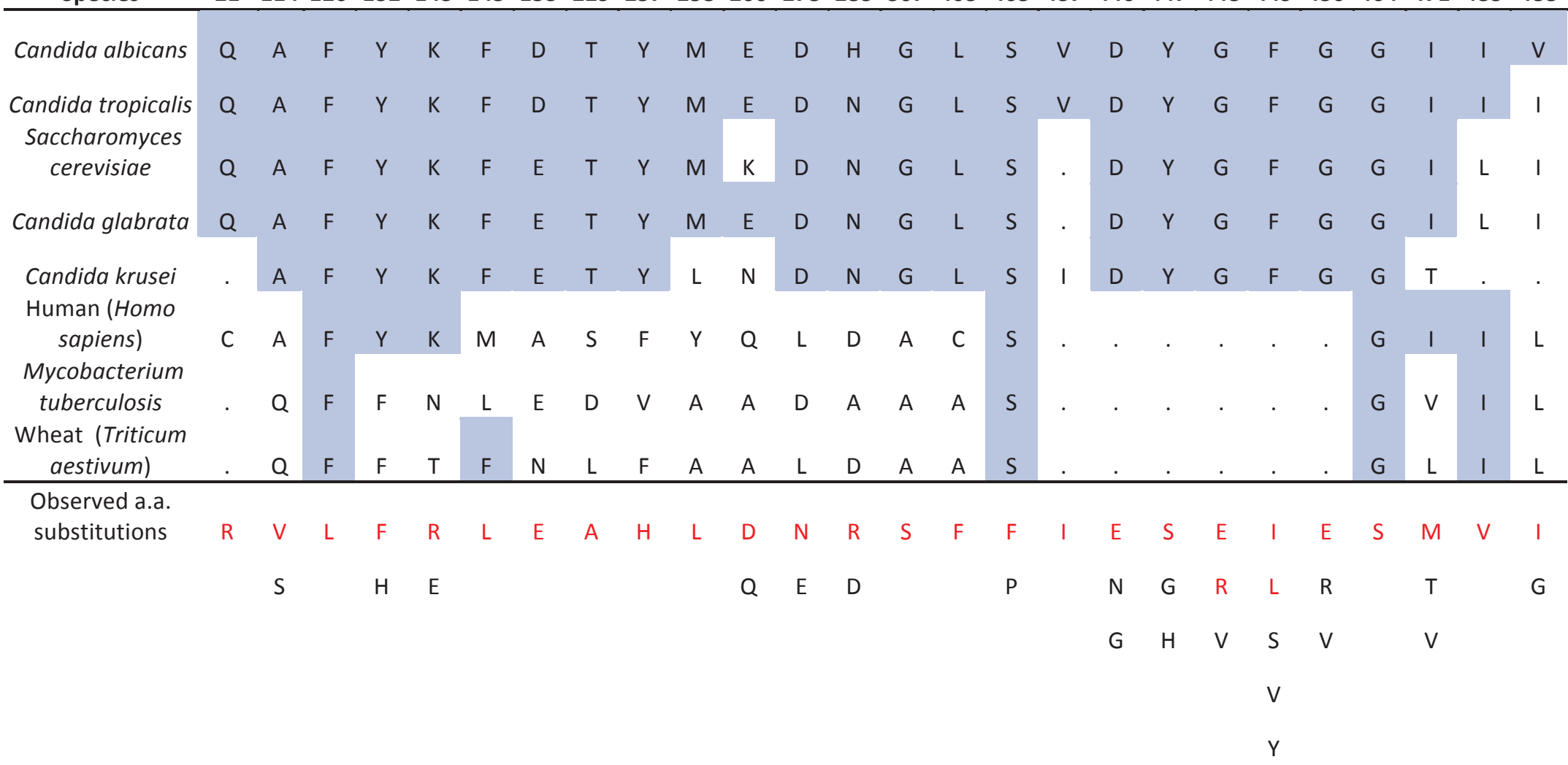


observed for M258L and D278N, which both independently occurred with a G464S mutation resulting in a twofold and a fourfold increase in fluconazole MICs, respectively, over that which was observed for G464S alone. Neither the M258L nor the D278N modifications have been described previously in the literature. D278E was previously shown to occur in azole resistant isolates however, the exchange of an aspartic acid (D) for a glutamic acid (E) may be a more conserved substitution when compared to the asparagine $(\mathrm{N})$ modification observed in our collection (Table 4.3) (111).

Not all mutations investigated contributed to azole resistance. ERG11 is observed in our studies and others to be highly permissive to genetic polymorphisms. The most common mutation observed in this collection was E266D $(n=10)$ and was always recovered with an additional substitution. In this study, I investigated the E266D substitution when combined with K143R, F145L and G464S independently. In all three cases, the addition of the E266D polymorphism did not confer additional effects on azole resistance beyond that which was observed for K143R, F145L and G464S alone. In the current study, I sequenced only ERG11 alleles from isolates exhibiting decreased susceptibilities to fluconazole. It is most likely that mutations in ERG11 occur in both azole-susceptible and resistant isolates that do not affect azole resistance. Another possibility is that mutations may arise in conjunction with mutations creating azole resistance that also affect fitness. Previous work has shown that specific ERG11 mutations affect fitness and catalytic activity of the enzyme (166, 201). Further investigation of how ERG11 mutations affect fitness is warranted.

One isolate contained a Q21L amino acid substitution in one ERG11 allele with a wild-type ERG11 allele. In membrane bound lanosterol demethylases, the far N-terminal region acts as a transmembrane portion of the enzyme that anchors this enzyme to the endoplasmic reticulum (38). This is the only mutation that I recovered in the far Nterminal region, and it was expressed with a wild-type allele. When expressed in SC5314, I did not observe a change in any azole susceptibilities at 48 hours. This data suggests that mutations occurring near the putative transmembrane region of the enzyme may not interfere with enzyme-substrate interaction.

Clearly, the susceptibility of clinical isolates is a product of the interplay of multiple mechanisms of resistance. Mutations in the ERG11 genes have been shown to be a significant and prevalent mechanism of resistance in C. albicans. Notably, 19 clinical isolates carried ERG11 mutations in addition to Upc2 GOF mutations. It is probable that the combination of these two mechanisms have a combinatorial effect on azole susceptibility. Our data clearly demonstrate that many ERG11 mutations result in fluconazole resistance, but most are not as significant when tested against voriconazole or itraconazole. Itraconazole, in particular seems less effected by ERG11 mutations which produce significant resistance to fluconazole. Despite this general observation, I have identified a specific combination of amino acid substitutions that significantly reduce itraconazole and voriconazole susceptibility. The association of clinically derived mutations in ERG11 resulting in decreased azole susceptibility are of clinical importance. In this study, I limited our characterization to specific mutant ERG11 alleles. Further 
characterization of clinically derived ERG11 mutant alleles to azole resistance is needed help guide antifungal pharmacotherapy and assist with future antifungal development. 


\section{CHAPTER 5. DISCUSSION AND CONCLUSIONS}

\section{GENERAL DISSERTATION OVERVIEW}

In Chapter 2, I describe the characterization of known resistance mechanisms in a large group of clinical C. albicans isolates that were enriched for decreased fluconazole susceptibility. A transcriptional profile for each isolate was created for genes known to cause azole resistance such as ERG11,CDR1, CDR2 and $M D R 1$. Stratified into groups based on fluconazole susceptible vs. resistant phenotypes (MIC $\geq 8$ ), transcription profiles of genes known in azole resistance were then compared.

As $C D R 1$ and $C D R 2$ are transcriptionally co-regulated by Tac1, it was not surprising to observe the coordinate expression of these transporters. Increased expression of $C D R 1$ was observed in $77 \%$ of isolates with increased resistance to fluconazole as opposed to the overexpression of $M D R 1$, which encompassed only $21 \%$. There was no correlation between transcriptional expression of the ABC transporters and the MFS transporter $M D R 1$ in this group. This observation may be due to specific fitness costs associated with the overexpression of both these transporters in one isolate. In 2012, Sasse et al systematically investigated the effects of GOF mutations in the transcriptional regulators $M R R 1, T A C 1, U P C 2$, and also mutations in ERG11, on associated fitness costs in $C$. albicans (166). In this work, the authors demonstrated that although combinations of mechanisms of drug resistance resulted in a step-wise acquisition of high level resistance, there was also an associated decrease in fitness when tested in non-selective conditions in vitro and in vivo. Mutations associated with the greatest decrease in fitness were the combination of a GOF mutation in TACl and MRRI in isolates constructed to carry two mechanisms of resistance. The addition of a GOF UPC2 allele resulted in a further decrease in fitness. In the absence of any compensatory mutations that might arise in a clinical isolate, this work demonstrated that loss of the wild-type regulation of gene expression is associated with a decrease in fitness. I believe these fitness costs may explain why simultaneous overexpression of $M D R 1$ and $C D R 1 / C D R 2$ are not observed in this group of clinical isolates.

In addition to efflux transporter expression, I also measured the transcriptional profile of ERG11, whose gene product is the target of azole antifungals. These data showed increased expression of ERG11 occurred in $75 \%$ of our clinical isolate collection. For those that overexpressed ERG11 when compared to fluconazole-susceptible isolates, I sequenced a known regulator of ergosterol biosynthesis UPC2. Previous to this study, only three distinct $U P C 2$ GOF mutations had been identified to occur clinically. Our sequenced data introduced five previously undescribed GOF mutations in UPC2 and also established that these mutations occurred frequently in azole-resistant isolates.

As has been described for activating mutations in zinc-cluster transcription factors in $S$. cerevisiae, all of the mutations observed in this study occurred in the C-terminal putative activation domain of CaUPC2. In C. albicans, UPC2 is orthologous to UPC2 and ECM22 in S. cerevisiae, which regulate ergosterol biosynthesis and uptake of 
exogenous sterols. Both transcription factors are localized to the intracellular membrane of the endoplasmic reticulum but under sterol-depleted activating conditions, they localize around the nucleus (115). Gain-of-function mutations have been identified in both ScUPC2 and ScECM22 and in each instance, mutations identified occur in the Cterminal activation domain (197). There are two theories that describe how GOF mutations result in constitutive activation of UPC2. First, mutations in the $\mathrm{C}$-terminus may interfere with the transmembrane region of the protein resulting in constitutive localization to the nucleus and constitutive activation of target genes. The second theory is that mutations in the C-terminal area could relieve Upc 2 of a repressor that would otherwise keep Upc2 in an inactive state in non-activating conditions (177).

The most widely characterized zinc-cluster transcription factor in yeast is Gal4. Gal4 regulates genes involved in the transport and metabolism of galactose (20). As has been shown in UPC2 localization studies in S. cerevisiae, Gal4 is localized to the cytoplasm and upon activation, is directed towards and imported into the nucleus. Gal4 is inhibited by repressor protein Gal80 in the absence of galactose. Previous work has shown that the carboxy-terminal 28 amino acids are responsible for the sequence specific interaction between these two proteins (161). A single amino acid substitution in this region is sufficient to constitutively activate target genes by relieving interaction of Gal4 with Gal80 (161). Notably, a mutation in Gal80 has been shown to compensate for a GOF mutation in Gal4, resulting in a wild-type phenotype. Adding another level of regulation in Gal4 activation is the detection of mutant Gal3 proteins that show altered Gal80binding characteristics and result in constitutive activation of Gal4 (14).

A repressor protein for $U P C 2$ or $E C M 22$ has not been identified in either $S$. cerevisiae or in $C$. albicans but it is plausible that these mutations interfere with this interaction. In addition to relief of a repressor, post-transcriptional modifications occur with Gal4 by the phosphorylation of a series of C-terminal serine residues which are thought to "fine tune" activation and may regulate ubiquitin degradation (160). Activating mutations observed in $\mathrm{CaUpc} 2$ have not been shown to occur on any serine residues but using an online phosphorylation site identifier (http://www.cbs.dtu.dk/services/NetPhos/), highlights potential phosphorylation sites identified at positions S469, S533 and S575.

In strains constructed to carry a GOF allele in $U P C 2$, I observed that homozygous replacement of the GOF UPC2 allele showed a greater effect on azole susceptibility than mutations expressed with a wild-type allele. This is consistent to what is observed in GOF mutations in TACl and MRRl. When comparing genome-wide transcriptional analysis of four distinct UPC2 GOF mutations I observed a core set of genes to be upregulated. Expectedly, among these genes were those involved in oxidoreductase activity and those involved in ergosterol biosynthesis. 


\section{Summary and discussion of Chapter 3}

In Chapter 3 I explored the hypothesis that mutations in NDT80 might result in overexpression of ERG11 in azole resistant isolates that do not carry mutations in UPC2. $\mathrm{Ndt} 80$ is a transcriptional regulator that in $C$. albicans has been shown to regulate ERG11 expression. ERG11 is a direct target of $\mathrm{Ndt} 80$ and its disruption results in reduced ERG11 expression and increased azole susceptibility. Clinical isolates 945 and1619 are genetically matched isolates comprised of a susceptible and resistant constituent, in which the resistant isolate overexpresses ERG11 and does not carry mutations in UPC2. I therefore sequenced NDT80 in both isolates and found several mutations to be present. Suspecting these mutations as being the cause of ERG11 overexpression in these isolates, I expressed mutant NDT80 alleles derived from the fluconazole-susceptible and -resistant isolates in SC5314. I did not observe a change in fluconazole resistance or ERG11 expression in strains carrying mutant NDT80 alleles. While this does not preclude the involvement of mutations in NDT80 in azole resistance, such mutations do not explain the overexpression of ERG11 in these clinical isolates.

\section{Summary and discussion of Chapter 4}

In Chapter 4, I characterized clinically derived mutations in the ERG11 gene and their effects on the activity of a panel of structurally different azole antifungals. I was able to demonstrate that distinct amino acid substitutions in Erg11 produced a variable increase in resistance to fluconazole, itraconazole and voriconazole. I also observed trends to placement of the resulting amino acid substitutions in the enzyme and the level of resulting resistance to fluconazole.

Both voriconazole and itraconazole susceptibilities were not as affected by mutations in ERG11 when compared to fluconazole. It is likely that structural differences resulting in stronger binding of newer generation azoles make them less vulnerable to the effects of ERG11 mutations. Itraconazole seemed to be the least susceptible to ERG11 mutations, with only the F145L + Y132F combination resulting in a significant increase in MIC. Although itraconazole retains its activity against ERG11 mutations and previous work has shown that itraconazole is not a substrate for Mdr1 efflux transport, the utility of this agent in the treatment of invasive candidiasis is wanting due to its erratic bioavailability and the lack of an intravenous formulation.

As discussed earlier, despite the low sequence homology of Cyp51 enzymes between species, the secondary structure is highly conserved and specific amino acid positions are conserved within fungal organisms and even between kingdoms. Its logical that as these positions are conserved between species, their role in the structure and function of Cyp51 likely important. In this collection, we observed and characterized mutations occurring both in conserved and non-conserved positions in the Cyp51 sequence. A general observation was that amino acid substitutions occurring in conserved positions resulted in fluconazole resistance. The exception to this observation was the Q21L substitution, which is conserved in fungal species yet did not result in increased MICs to azoles. 
Likewise, amino acid substitutions occurring in non-conserved positions, such as E266D did not affect fluconazole susceptibility and are not associated with azole-resistant isolates in this study or in previous publications (122). Over 150 missense mutations in ERG11 have been documented in the literature and characterizing mutations as I have by constructing strains that carry these mutations is laborious and slow. Generally, mutations characterized in this study that result in a change in azole resistance are those that arise in clinically resistant isolates and are in conserved positions in the Cyp51 enzyme. As sequencing can now be efficiently and cost-effectively performed on a large scale, correlating mutations to azole resistance can be done statistically in the future once sufficient data is available.

Although several homology models for fungal Cyp51 structures have been published, a crystal structure for this enzyme is not public. Many publications on fungal Cyp51 structure cite the difficulty of obtaining a crystal for membrane bound enzymes. Despite this difficulty, X-ray structures for 25 membrane bound Cyp51 enzymes are available, including that for humans(38). As Cyp51 for C. albicans, as well as other fungal species, is the most important and exploited target for antifungals, the addition of a CaCyp51 crystal structure would aid in the development of better azole antifungals.

\section{DISCUSSION OF METHODS}

The studies discussed in this dissertation describe the use of a variety of molecular techniques to quantify gene transcription involved in mechanisms of resistance and the construction of strains that carry mutant genes derived from resistant isolates in a wildtype background strain. With these constructed strains, I was able to specifically identify the specific contribution to azole resistance and to identify key determinants of azole resistance in this species.

\section{Gene-quantification}

To quantify genes involved in azole resistance, I used quantitative real-time PCR (RT-PCR), which is the standard method of gene quantification. This method has several advantages over other methods of gene quantification due to its increased sensitivity and quantitative capabilities. To quantify gene expression, I used the Comparative $C_{t}\left(2^{-\Delta \Delta C t}\right)$ method. Using this model, I can calculate changes in gene expression as a relative fold difference between an experimental and a calibrator sample. This method requires similar amplification kinetics of both the target and reference gene because different efficiencies will generate errors with this method. Therefore, for each gene measured, a validation assay using serial dilutions of cDNA target are assayed for each gene of interest and the results are plotted with the log-input concentration for each dilution on the $\mathrm{x}$-axis, and the difference for $\mathrm{C}_{\mathrm{t}}$ dilution on the $\mathrm{y}$-axis. This particular collection was comprised of a large number of unrelated clinical isolates. Most expression experiments of this nature are performed with genetically match-paired isolates that differ only in azolesusceptibility phenotypes. As none of our isolates are related, I averaged the gene 
expression data from azole susceptible isolates to compared expression of genes of interest to our resistant isolates.

Studies have shown that genes involved in azole resistance are regulated at the transcriptional level in response to azole drugs, and not to gene duplication events (105). Although transcription of specific genes is correlated with azole resistance, using methods that only quantify RNA could be a limitation to this work. Manoharlal et al. demonstrated that in addition to increased transcription initiation of CDR 1, CDR 1 mRNA stability half-life was threefold higher in an azole-resistant strain when compared to an azole-susceptible counterpart (112). Although correlating gene expression with resistance in $C$. albicans is standard in the literature, this technique neglects regulation that could occur post-transcriptionally or post-translationally that could be contributing to an azoleresistant phenotype.

\section{Genetic manipulation in C. albicans}

The SAT1-flipper technique was utilized to perform targeted gene inactivation and replacement. The $S A T 1$ flipping strategy allows for genetic manipulation to occur in prototrophic $C$. albicans strains using a dominant drug selection marker that is recyclable, which is important in diploid species(156). This method uses a CaSAT1-selectable marker which enables resistance to the aminoglycoside nourseothricin and also a CaFLP gene, which encodes a site-specific recombinase for cassette excision and selection marker recycling. Previous to the technique, targeted gene disruption occurred in specific auxotrophic background strains using nutritional markers for the selection of transformants. However, use of auxotrophic markers made it difficult to interpret mutant phenotypes as these genes were shown to modulate processes such as virulence.

Although the $S A T 1$ flipper method has resolved difficulties associated with the use of auxotrophic strains, there are other complications associated with genetic manipulation of C. albicans. As stated in the introduction, genetic plasticity in the C. albicans genome is well described and development of aneuploidy and chromosomal rearrangements occur readily under a wide variety of events including antifungal drug exposure. Aneuploidies of selected chromosomes are not uncommon in laboratory strains $(96,172)$.

Chromosomal aneuploidies and truncations have been described in laboratory strains as a result of genetic modification like what is described in this dissertation. It is clear that detection of chromosomal ploidy is important in this species. As we do not have a coteffective mechanism to routinely detect this in our lab, I have constructed duplicates of each strain to ensure reproducibility of the phenotypes I have observed in these mutant strains.

\section{Measuring antifungal susceptibility}

To measure antifungal susceptibility, we used a modified version of the Clinical Laboratory and Standards Institute microbroth dilution method. The modification of this 
protocol is the addition of $2 \%$ dextrose to RPMI media, which has been observed to decrease the amount of trailing associated with azole fungistatic activity in this species. Reducing the amount of trailing is important for interpretation of data. In addition to standard broth dilution methods, we occasionally used azole treated E-test strips (Biomerieux) as these interpretations of MICs are less subjective. At times, instead of RPMI, we test azole susceptibilities in rich YPD media in order reveal smaller changes resulting from certain genes. Potential drawbacks of using YPD media for susceptibility testing are that media varies based on preparation and one cannot make associations of susceptibility to clinical breakpoints, as those are performed in RPMI.

The ability to incorporate exogenous sterols into the fungal cell membrane has been shown for S. cerevisiae and C. glabrata but has not yet been shown for C. albicans. If this process occurs, it may be that testing MICs in media without exogenous sterols may underrepresent resistance phenotypes. If a particular isolate has an increased ability to incorporate sterols from the environment, inhibition of ergosterol synthesis could be bypassed. As yeast extract is a component of YPD, this may be partly contributing the increased growth and higher MICs resulting from susceptibility testing in this media.

\section{OVERALL THEME AND CONCLUSIONS}

Throughout the research presented in this dissertation, there is one recurring theme which deserves attention. The work presented here is the largest comparison of resistance mechanisms that have been defined in a group of azole-resistant clinical isolates of $C$. albicans. Prior to this work, the primary focus of azole resistance was on efflux transporters $C D R 1, C D R 2$ and $M D R 1$. It was not surprising that overexpression of efflux transporters was well represented in this collection. Notably, expression data in this work revealed the considerable involvement of ERG11 overexpression which had previously taken a back seat to the involvement of efflux transporters. Overexpression of ERG11 seems to be something specific for $C$. albicans, as smaller population studies have not shown increased ERG11 expression to be prevalent in C. glabrata or C. dubliniensis. Also in this group, we defined the significance of individual mutant $E R G 11$ alleles on azole resistance. Numerous sequencing data has demonstrated $E R G 11$ is highly tolerant of mutations in both azole-susceptible and resistant isolates. These data corroborated with sequencing data for this collection. Large scale phenotypic characterization of these mutations done in this work demonstrated ERG11 mutations were a prevalent contributor to azole resistance in C. albicans.

\section{FUTURE DIRECTIONS}

The long term goal of our research is to improve the treatment of Candida infections by understanding the molecular basis of azole resistance. Ultimately, part of goal will be to develop improved options for the treatment of candidiasis. Currently, there are only 4 systemic therapies available for the treatment of fungal disease. Despite the increasing need for new antifungal classes to treat candidiasis, antifungal drug discovery is limited 
by the detection of novel and specific antifungal targets. The azole class of antifungals is presently our only oral option for the treatment of systemic disease but as shown in this work, there are clinical isolates that have become highly resistant to these agents. In Chapter 4, I investigated several mutations in ERG11, some of which caused an increase in voriconazole and itraconazole susceptibility to the susceptible dose-dependent range. In this study, I was only able to characterize a subset ERG11 alleles observed, but there are probably specific mutant alleles, like those which contain 3 or 4 amino acid substitutions, which may confer a greater effect on azole resistance. The more information obtained on ERG11 mutations and their specific effect azole resistance may shed light on how to better target Erg11 either by construction of improved members of the azole class or by development of a new compound.

Although my contribution to characterizing ERG11-mediated azole resistance in $C$. albicans is nearly complete, there are areas in this project that warrant further exploration. I present here some future directions made possible from the groundwork prepared by these data.

\section{Whole genome sequencing to investigate $U P C$-independent mechanisms of $E R G 11$ - overexpression}

Our lab and others have observed ERG11 overexpression in isolates that do not contain GOF mutations in UPC2. In Chapter 3, I investigated the role of mutant NDT80 alleles derived from resistant isolates in ERG11 overexpression but was unable to demonstrate a role for this transcription factor in regulating azole resistance. The mechanism of ERG11 overexpression in these isolates remains unknown. The strategy of targeted gene sequencing based on genome-wide expression data or educated guesses are time consuming and largely yield negative results. As there are potentially several genes involved in azole resistance that have not been identified, investigations of this nature can now be undertaken on a genome-wide scale.

As transcription factors have played a primary role in many key drug resistance and pathogenic processes, exploring mutations that occur in these genes containing missense polymorphisms in resistant isolates will be an obvious and fruitful choice to investigate further. As someone who has spent considerable time exploring sequencing data of this type, I have further suggestions of possible genes and pathways to explore in future studies.

First, I recommend that in addition to mutations in ERG11, polymorphisms occurring in other components of the ergosterol biosynthesis pathway be explored. Studies have already shown that azole- and amphotericin B- resistant species of Candida display alternate sterol profiles. Additionally, as microarray data show an increased expression of genes important to ergosterol biosynthesis in response to chemical inhibition, I suspect that decreased ergosterol production resulting from mutations in the $E R G$ pathway may also result in increased ERG11 expression. 
A next generation sequencing study performed in a series of clinical $C$. glabrata isolates has already identified a gene mutation that arises after $F K S$ mutations that result in ameliorating fitness costs of echinocandin resistance (178). As similar fitness defects occur in azole-resistant $C$. albicans strains, detection of genes that restore fitness in clinical isolates would represent an additional mechanism of resistance in this species.

In the future, proteins required for pathogenicity in fungus may be considered a potential drug targeting strategy. One unit of chromatin is defined as 146bp of DNA wrapped around a histone complex comprised of two histone $\mathrm{H} 2 \mathrm{~A}-\mathrm{H} 2 \mathrm{~B}$ dimers and one histone $(\mathrm{H} 3-\mathrm{H} 4)_{2}$ tetramer(103). Histone proteins play a role in tightly packaging DNA into nucleosomes and association to a tightly wound chromatin is associated with gene silencing. Data from genome-wide transcription profiles suggest that wide-spread transcriptional networks are activated in response to antifungals and pathogenesis, which suggests some level of chromatin regulating events (175). Specific histone modifications result in either the relaxing of the histone tail permitting access of transcriptional machinery to coding genes or tightening of the histone complex resulting in gene silencing. The role of various histone modification enzymes have already been investigated regarding pathogenesis and even antifungal resistance in Candida species as nicely reviewed by Lopes de Rosa and Kaufman (84). Identification of mutations that modulate chromatin structure in resistant isolates would further delineate drug resistance mechanisms and perhaps identify future drug targets.

\section{Employing genotypic testing as a tool to guide antifungal therapy}

As mentioned earlier, the CLSI guidelines have been recently modified for both azoles and echinocandins. Newer clinical breakpoints take into account species specific epidemiological cutoff values (ECV), resulting in species specific breakpoints to help guide therapy. Revising these breakpoints may allow us to detect Candida species and isolates that are a risk for the development of resistance. Although recent data show that C. albicans as a species remains largely susceptible to the azole class, this work demonstrates that azole resistance does arise in clinical isolates. As a means to quickly and efficiently guide treatment as well as to track resistance patterns in Candida species, genotypic data could potentially play a large role in guiding antifungal treatment in the future.

As an example of this type of analysis, Shields et. al described the relationship between echinocandin MICs and patient responses to therapy in C. glabrata (176). Authors extracted information from medical records (prior echinocandin exposure, duration of treatment, patient outcomes) and tied this with microbiologic data such as caspofungin susceptibilities and occurrence of $F K S$ mutations. This study produced a paradigm defining a method-specific caspofungin susceptibility threshold that was sensitive and specific enough to detect the presence of $F K S$ mutations in C. glabrata. Of direct clinical importance, authors also identified prior echinocandin use and caspofungin MIC over the defined threshold (MIC $>0.25 \mu \mathrm{g} / \mathrm{mL}$ for E test) as the most sensitive predictors of treatment outcomes. 
In C. albicans, azole resistance in clinical isolates is multifactorial but there is extensive data defining key mutations in genes resulting in a resistant phenotype. In a similar manner, it would be significant to use these data to further define treatment paradigms in an effort to detect resistant organisms and to improve patient outcomes. 


\section{LIST OF REFERENCES}

1. 1981. Centers for Disease Control and Prevention. Pneumocystis pneumonia--Los Angeles. MMWR Morb Mortal Wkly Rep 30:250-2.

2. Achkar, J. M., and B. C. Fries. 2010. Candida infections of the genitourinary tract. Clin Microbiol Rev 23:253-73.

3. Agvald-Ohman, C., L. Klingspor, H. Hjelmqvist, and C. Edlund. 2008. Invasive candidiasis in long-term patients at a multidisciplinary intensive care unit: Candida colonization index, risk factors, treatment and outcome. Scand J Infect Dis 40:145-53.

4. Alonso-Monge, R., F. Navarro-Garcia, G. Molero, R. Diez-Orejas, M. Gustin, J. Pla, M. Sanchez, and C. Nombela. 1999. Role of the mitogen-activated protein kinase Hog $1 \mathrm{p}$ in morphogenesis and virulence of Candida albicans. $\mathrm{J}$ Bacteriol 181:3058-68.

5. Alvarez-Rueda, N., A. Fleury, F. Morio, F. Pagniez, L. Gastinel, and P. Le Pape. 2011. Amino acid substitutions at the major insertion loop of Candida albicans sterol 14alpha-demethylase are involved in fluconazole resistance. PLoS One 6:e21239.

6. Ampel, N. M. 1996. Emerging disease issues and fungal pathogens associated with HIV infection. Emerg Infect Dis 2:109-16.

7. Anderson, M. R., K. Klink, and A. Cohrssen. 2004. Evaluation of vaginal complaints. JAMA 291:1368-79.

8. Andes, D., and M. van Ogtrop. 2000. In vivo characterization of the pharmacodynamics of flucytosine in a neutropenic murine disseminated candidiasis model. Antimicrob Agents Chemother 44:938-42.

9. Ashley, E. D., R. Drew, M. Johnson, R. Danna, D. Dabrowski, V. Walker, M. Prasad, B. Alexander, G. Papadopoulos, and J. Perfect. 2012. Cost of Invasive Fungal Infections in the Era of New Diagnostics and Expanded Treatment Options. Pharmacotherapy.

10. Barnett, J. A. 2004. A history of research on yeasts 8: taxonomy. Yeast 21:114193.

11. Beernaert, L. A., F. Pasmans, L. Van Waeyenberghe, F. Haesebrouck, and A. Martel. 2010. Aspergillus infections in birds: a review. Avian Pathol 39:325-31.

12. Bennett, R. J., and A. D. Johnson. 2003. Completion of a parasexual cycle in Candida albicans by induced chromosome loss in tetraploid strains. EMBO J 22:2505-15.

13. Bhaumik, S. R., T. Raha, D. P. Aiello, and M. R. Green. 2004. In vivo target of a transcriptional activator revealed by fluorescence resonance energy transfer. Genes Dev 18:333-43.

14. Blank, T. E., M. P. Woods, C. M. Lebo, P. Xin, and J. E. Hopper. 1997. Novel Gal3 proteins showing altered Gal80p binding cause constitutive transcription of Gal4p-activated genes in Saccharomyces cerevisiae. Mol Cell Biol 17:2566-75.

15. Brammer, K. W., A. J. Coakley, S. G. Jezequel, and M. H. Tarbit. 1991. The disposition and metabolism of [14C]fluconazole in humans. Drug Metab Dispos 19:764-7. 
16. Brammer, K. W., P. R. Farrow, and J. K. Faulkner. 1990. Pharmacokinetics and tissue penetration of fluconazole in humans. Rev Infect Dis 12 Suppl 3:S31826.

17. Bruno, V. M., and A. P. Mitchell. 2005. Regulation of azole drug susceptibility by Candida albicans protein kinase CK2. Mol Microbiol 56:559-73.

18. Butler, G., M. D. Rasmussen, M. F. Lin, M. A. Santos, S. Sakthikumar, C. A. Munro, E. Rheinbay, M. Grabherr, A. Forche, J. L. Reedy, I. Agrafioti, M. B. Arnaud, S. Bates, A. J. Brown, S. Brunke, M. C. Costanzo, D. A. Fitzpatrick, P. W. de Groot, D. Harris, L. L. Hoyer, B. Hube, F. M. Klis, C. Kodira, N. Lennard, M. E. Logue, R. Martin, A. M. Neiman, E. Nikolaou, M. A. Quail, J. Quinn, M. C. Santos, F. F. Schmitzberger, G. Sherlock, P. Shah, K. A. Silverstein, M. S. Skrzypek, D. Soll, R. Staggs, I. Stansfield, M. P. Stumpf, P. E. Sudbery, T. Srikantha, Q. Zeng, J. Berman, M. Berriman, J. Heitman, N. A. Gow, M. C. Lorenz, B. W. Birren, M. Kellis, and C. A. Cuomo. 2009. Evolution of pathogenicity and sexual reproduction in eight Candida genomes. Nature 459:657-62.

19. Casadevall, A. 2012. Fungi and the rise of mammals. PLoS Pathog 8:e1002808.

20. Chan, C. K., and D. A. Jans. 1999. Synergy of importin alpha recognition and DNA binding by the yeast transcriptional activator GAL4. FEBS Lett 462:221-4.

21. Chen, C. G., Y. L. Yang, H. I. Shih, C. L. Su, and H. J. Lo. 2004. CaNdt80 is involved in drug resistance in Candida albicans by regulating CDR1. Antimicrob Agents Chemother 48:4505-12.

22. Coste, A., V. Turner, F. Ischer, J. Morschhauser, A. Forche, A. Selmecki, J. Berman, J. Bille, and D. Sanglard. 2006. A mutation in Tac1p, a transcription factor regulating CDR1 and CDR2, is coupled with loss of heterozygosity at chromosome 5 to mediate antifungal resistance in Candida albicans. Genetics 172:2139-56.

23. Coste, A. T., M. Karababa, F. Ischer, J. Bille, and D. Sanglard. 2004. TAC1, transcriptional activator of CDR genes, is a new transcription factor involved in the regulation of Candida albicans ABC transporters CDR1 and CDR2. Eukaryot Cell 3:1639-52.

24. Cournia, Z., G. M. Ullmann, and J. C. Smith. 2007. Differential effects of cholesterol, ergosterol and lanosterol on a dipalmitoyl phosphatidylcholine membrane: a molecular dynamics simulation study. J Phys Chem B 111:1786801.

25. Courtney, R., E. Radwanski, J. Lim, and M. Laughlin. 2004. Pharmacokinetics of posaconazole coadministered with antacid in fasting or nonfasting healthy men. Antimicrob Agents Chemother 48:804-8.

26. Courtney, R., Sansone-Parsons, A., Devlin, D., Soni, P., Laughlin, M., Simon, J. 2004. P-Glycoprotein (P-gp) expression and gentype: exploratory analysis of posaconazole (POS) in healthy volunteers. . Intrsci Conf Antimicrob Agents Chemother Abstract A-40.

27. Courtney, R., D. Wexler, E. Radwanski, J. Lim, and M. Laughlin. 2004. Effect of food on the relative bioavailability of two oral formulations of posaconazole in healthy adults. Br J Clin Pharmacol 57:218-22. 
28. Crowley, J. H., F. W. Leak, Jr., K. V. Shianna, S. Tove, and L. W. Parks. 1998. A mutation in a purported regulatory gene affects control of sterol uptake in Saccharomyces cerevisiae. J Bacteriol 180:4177-83.

29. Crump, J. A., and P. J. Collignon. 2000. Intravascular catheter-associated infections. Eur J Clin Microbiol Infect Dis 19:1-8.

30. Cryan, P. M., C. U. Meteyer, D. S. Blehert, J. M. Lorch, D. M. Reeder, G. G. Turner, J. Webb, M. Behr, M. Verant, R. E. Russell, and K. T. Castle. 2013. Electrolyte depletion in white-nose syndrome bats. J Wildl Dis 49:398-402.

31. Cryan, P. M., C. U. Meteyer, J. G. Boyles, and D. S. Blehert. 2013. White-nose syndrome in bats: illuminating the darkness. BMC Biol 11:47.

32. Csank, C., K. Schroppel, E. Leberer, D. Harcus, O. Mohamed, S. Meloche, D. Y. Thomas, and M. Whiteway. 1998. Roles of the Candida albicans mitogenactivated protein kinase homolog, Cek1p, in hyphal development and systemic candidiasis. Infect Immun 66:2713-21.

33. Dalle, F., B. Wachtler, C. L'Ollivier, G. Holland, N. Bannert, D. Wilson, C. Labruere, A. Bonnin, and B. Hube. Cellular interactions of Candida albicans with human oral epithelial cells and enterocytes. Cell Microbiol 12:248-71.

34. Davies, B. S., and J. Rine. 2006. A role for sterol levels in oxygen sensing in Saccharomyces cerevisiae. Genetics 174:191-201.

35. de Leon, E. M., S. J. Jacober, J. D. Sobel, and B. Foxman. 2002. Prevalence and risk factors for vaginal Candida colonization in women with type 1 and type 2 diabetes. BMC Infect Dis 2:1.

36. de Micheli, M., J. Bille, C. Schueller, and D. Sanglard. 2002. A common drugresponsive element mediates the upregulation of the Candida albicans $A B C$ transporters CDR1 and CDR2, two genes involved in antifungal drug resistance. Mol Microbiol 43:1197-214.

37. Delgado, A. C., R. de Jesus Pedro, F. H. Aoki, M. R. Resende, P. Trabasso, A. L. Colombo, M. S. de Oliveira, Y. Mikami, and M. L. Moretti. 2009. Clinical and microbiological assessment of patients with a long-term diagnosis of human immunodeficiency virus infection and Candida oral colonization. Clin Microbiol Infect 15:364-71.

38. Denisov, I. G., A. Y. Shih, and S. G. Sligar. 2012. Structural differences between soluble and membrane bound cytochrome P450s. J Inorg Biochem 108:150-8.

39. Deray, G. 2002. Amphotericin B nephrotoxicity. J Antimicrob Chemother 49 Suppl 1:37-41.

40. Desta, Z., X. Zhao, J. G. Shin, and D. A. Flockhart. 2002. Clinical significance of the cytochrome P450 2C19 genetic polymorphism. Clin Pharmacokinet 41:913-58.

41. Diez-Orejas, R., G. Molero, F. Navarro-Garcia, J. Pla, C. Nombela, and M. Sanchez-Perez. 1997. Reduced virulence of Candida albicans MKC1 mutants: a role for mitogen-activated protein kinase in pathogenesis. Infect Immun 65:833-7.

42. Dore, G. J., and D. A. Cooper. 2006. HAART's first decade: success brings further challenges. Lancet 368:427-8. 
43. Dunkel, N., J. Blass, P. D. Rogers, and J. Morschhauser. 2008. Mutations in the multi-drug resistance regulator MRR1, followed by loss of heterozygosity, are the main cause of MDR1 overexpression in fluconazole-resistant Candida albicans strains. Mol Microbiol 69:827-40.

44. Dunkel, N., T. T. Liu, K. S. Barker, R. Homayouni, J. Morschhauser, and P. D. Rogers. 2008. A gain-of-function mutation in the transcription factor Upc $2 p$ causes upregulation of ergosterol biosynthesis genes and increased fluconazole resistance in a clinical Candida albicans isolate. Eukaryot Cell 7:1180-90.

45. Ezzet, F., D. Wexler, R. Courtney, G. Krishna, J. Lim, and M. Laughlin. 2005. Oral bioavailability of posaconazole in fasted healthy subjects: comparison between three regimens and basis for clinical dosage recommendations. Clin Pharmacokinet 44:211-20.

46. Fanning, S., and A. P. Mitchell. Fungal biofilms. PLoS Pathog 8:e1002585.

47. Feigal, D. W., M. H. Katz, D. Greenspan, J. Westenhouse, W. Winkelstein, Jr., W. Lang, M. Samuel, S. P. Buchbinder, N. A. Hessol, A. R. Lifson, and et al. 1991. The prevalence of oral lesions in HIV-infected homosexual and bisexual men: three San Francisco epidemiological cohorts. AIDS 5:519-25.

48. Finkel, J. S., and A. P. Mitchell. Genetic control of Candida albicans biofilm development. Nat Rev Microbiol 9:109-18.

49. Fisher, J. F., C. L. Newman, and J. D. Sobel. 1995. Yeast in the urine: solutions for a budding problem. Clin Infect Dis 20:183-9.

50. Flowers, S. A., K. S. Barker, E. L. Berkow, G. Toner, S. G. Chadwick, S. E. Gygax, J. Morschhauser, and P. D. Rogers. 2012. Gain-of-function mutations in UPC2 are a frequent cause of ERG11 upregulation in azole-resistant clinical isolates of Candida albicans. Eukaryot Cell 11:1289-99.

51. Forche, A., K. Alby, D. Schaefer, A. D. Johnson, J. Berman, and R. J. Bennett. 2008. The parasexual cycle in Candida albicans provides an alternative pathway to meiosis for the formation of recombinant strains. PLoS Biol 6:e110.

52. Forche, A., P. T. Magee, A. Selmecki, J. Berman, and G. May. 2009. Evolution in Candida albicans populations during a single passage through a mouse host. Genetics 182:799-811.

53. Franz, R., M. Ruhnke, and J. Morschhauser. 1999. Molecular aspects of fluconazole resistance development in Candida albicans. Mycoses 42:453-8.

54. Frohner, I. E., C. Bourgeois, K. Yatsyk, O. Majer, and K. Kuchler. 2009. Candida albicans cell surface superoxide dismutases degrade host-derived reactive oxygen species to escape innate immune surveillance. Mol Microbiol 71:240-52.

55. Gao, C., L. Wang, E. Milgrom, and W. C. Shen. 2004. On the mechanism of constitutive Pdr1 activator-mediated PDR5 transcription in Saccharomyces cerevisiae: evidence for enhanced recruitment of coactivators and altered nucleosome structures. J Biol Chem 279:42677-86.

56. Garaizar, J., S. Brena, J. Bikandi, A. Rementeria, and J. Ponton. 2006. Use of DNA microarray technology and gene expression profiles to investigate the pathogenesis, cell biology, antifungal susceptibility and diagnosis of Candida albicans. FEMS Yeast Res 6:987-98. 
57. Garey, K. W., M. Rege, M. P. Pai, D. E. Mingo, K. J. Suda, R. S. Turpin, and D. T. Bearden. 2006. Time to initiation of fluconazole therapy impacts mortality in patients with candidemia: a multi-institutional study. Clin Infect Dis 43:25-31.

58. Ghannoum, M. A., R. J. Jurevic, P. K. Mukherjee, F. Cui, M. Sikaroodi, A. Naqvi, and P. M. Gillevet. 2010. Characterization of the oral fungal microbiome (mycobiome) in healthy individuals. PLoS Pathog 6:e1000713.

59. Ghannoum, M. A., and L. B. Rice. 1999. Antifungal agents: mode of action, mechanisms of resistance, and correlation of these mechanisms with bacterial resistance. Clin Microbiol Rev 12:501-17.

60. Ghosal, A., N. Hapangama, Y. Yuan, J. Achanfuo-Yeboah, R. Iannucci, S. Chowdhury, K. Alton, J. E. Patrick, and S. Zbaida. 2004. Identification of human UDP-glucuronosyltransferase enzyme(s) responsible for the glucuronidation of ezetimibe (Zetia). Drug Metab Dispos 32:314-20.

61. Ghosh, S., D. H. Navarathna, D. D. Roberts, J. T. Cooper, A. L. Atkin, T. M. Petro, and K. W. Nickerson. 2009. Arginine-induced germ tube formation in Candida albicans is essential for escape from murine macrophage line RAW 264.7. Infect Immun 77:1596-605.

62. Gow, N. A., and B. Hube. Importance of the Candida albicans cell wall during commensalism and infection. Curr Opin Microbiol 15:406-12.

63. Gray, K. C., D. S. Palacios, I. Dailey, M. M. Endo, B. E. Uno, B. C. Wilcock, and M. D. Burke. 2012. Amphotericin primarily kills yeast by simply binding ergosterol. Proc Natl Acad Sci U S A 109:2234-9.

64. Gray, R. R., A. J. Tatem, J. A. Johnson, A. V. Alekseyenko, O. G. Pybus, M. A. Suchard, and M. Salemi. 2011. Testing spatiotemporal hypothesis of bacterial evolution using methicillin-resistant Staphylococcus aureus ST239 genome-wide data within a bayesian framework. Mol Biol Evol 28:1593-603.

65. Groll, A. H., J. C. Gea-Banacloche, A. Glasmacher, G. Just-Nuebling, G. Maschmeyer, and T. J. Walsh. 2003. Clinical pharmacology of antifungal compounds. Infect Dis Clin North Am 17:159-91, ix.

66. Heilmann, C. J., S. Schneider, K. S. Barker, P. D. Rogers, and J. Morschhauser. 2010. An A643T mutation in the transcription factor Upc2p causes constitutive ERG11 upregulation and increased fluconazole resistance in Candida albicans. Antimicrob Agents Chemother 54:353-9.

67. Hepworth, S. R., H. Friesen, and J. Segall. 1998. NDT80 and the meiotic recombination checkpoint regulate expression of middle sporulation-specific genes in Saccharomyces cerevisiae. Mol Cell Biol 18:5750-61.

68. Herbrecht, R., D. W. Denning, T. F. Patterson, J. E. Bennett, R. E. Greene, J. W. Oestmann, W. V. Kern, K. A. Marr, P. Ribaud, O. Lortholary, R. Sylvester, R. H. Rubin, J. R. Wingard, P. Stark, C. Durand, D. Caillot, E. Thiel, P. H. Chandrasekar, M. R. Hodges, H. T. Schlamm, P. F. Troke, and B. de Pauw. 2002. Voriconazole versus amphotericin B for primary therapy of invasive aspergillosis. N Engl J Med 347:408-15.

69. Hernday, A. D., S. M. Noble, Q. M. Mitrovich, and A. D. Johnson. Genetics and molecular biology in Candida albicans. Methods Enzymol 470:737-58. 
70. Hickman, M. A., G. Zeng, A. Forche, M. P. Hirakawa, D. Abbey, B. D. Harrison, Y. M. Wang, C. H. Su, R. J. Bennett, Y. Wang, and J. Berman. The 'obligate diploid' Candida albicans forms mating-competent haploids. Nature 494:55-9.

71. Hollenbach, E. 2008. To treat or not to treat--critically ill patients with candiduria. Mycoses 51 Suppl 2:12-24.

72. Holubar, K., and N. Wikonkal. 2010. David Gruby 1810-1898: unveiling of a portrait bust in his birthplace. Skinmed 8:294-5.

73. Hoot, S. J., R. P. Brown, B. G. Oliver, and T. C. White. 2010. The UPC2 promoter in Candida albicans contains two cis-acting elements that bind directly to Upc2p, resulting in transcriptional autoregulation. Eukaryot Cell 9:1354-62.

74. Hoot, S. J., A. R. Smith, R. P. Brown, and T. C. White. 2011. An A643V amino acid substitution in Upc2p contributes to azole resistance in wellcharacterized clinical isolates of Candida albicans. Antimicrob Agents Chemother 55:940-2.

75. Huh, W. K., J. V. Falvo, L. C. Gerke, A. S. Carroll, R. W. Howson, J. S. Weissman, and E. K. O'Shea. 2003. Global analysis of protein localization in budding yeast. Nature 425:686-91.

76. Hull, C. M., and A. D. Johnson. 1999. Identification of a mating type-like locus in the asexual pathogenic yeast Candida albicans. Science 285:1271-5.

77. Hwang, C. S., G. E. Rhie, J. H. Oh, W. K. Huh, H. S. Yim, and S. O. Kang. 2002. Copper- and zinc-containing superoxide dismutase $(\mathrm{Cu} / \mathrm{ZnSOD})$ is required for the protection of Candida albicans against oxidative stresses and the expression of its full virulence. Microbiology 148:3705-13.

78. Isoherranen, N., K. L. Kunze, K. E. Allen, W. L. Nelson, and K. E. Thummel. 2004. Role of itraconazole metabolites in CYP3A4 inhibition. Drug Metab Dispos 32:1121-31.

79. Jacobsen, I. D., D. Wilson, B. Wachtler, S. Brunke, J. R. Naglik, and B. Hube. Candida albicans dimorphism as a therapeutic target. Expert Rev Anti Infect Ther 10:85-93.

80. Johnson, A. 2003. The biology of mating in Candida albicans. Nat Rev Microbiol 1:106-16.

81. Johnson, L. B., and C. A. Kauffman. 2003. Voriconazole: a new triazole antifungal agent. Clin Infect Dis 36:630-7.

82. Jones, T., N. A. Federspiel, H. Chibana, J. Dungan, S. Kalman, B. B. Magee, G. Newport, Y. R. Thorstenson, N. Agabian, P. T. Magee, R. W. Davis, and S. Scherer. 2004. The diploid genome sequence of Candida albicans. Proc Natl Acad Sci U S A 101:7329-34.

83. Kadosh, D., and K. Struhl. 1997. Repression by Ume6 involves recruitment of a complex containing Sin 3 corepressor and Rpd3 histone deacetylase to target promoters. Cell 89:365-71.

84. Kaufman, D. A. 2012. "Getting to Zero": preventing invasive Candida infections and eliminating infection-related mortality and morbidity in extremely preterm infants. Early Hum Dev 88 Suppl 2:S45-9. 
85. Kelly, S. L., D. C. Lamb, and D. E. Kelly. 1999. Y132H substitution in Candida albicans sterol 14alpha-demethylase confers fluconazole resistance by preventing binding to haem. FEMS Microbiol Lett 180:171-5.

86. Kelly, S. L., D. C. Lamb, J. Loeffler, H. Einsele, and D. E. Kelly. 1999. The G464S amino acid substitution in Candida albicans sterol 14alpha-demethylase causes fluconazole resistance in the clinic through reduced affinity. Biochem Biophys Res Commun 262:174-9.

87. Kirk, P. 2008. Dictionary of the Fungi, 10th ed. CAB International, Wallingford.

88. Klein, R. S., C. A. Harris, C. B. Small, B. Moll, M. Lesser, and G. H. Friedland. 1984. Oral candidiasis in high-risk patients as the initial manifestation of the acquired immunodeficiency syndrome. N Engl J Med 311:354-8.

89. Klepser, M. E., E. J. Wolfe, R. N. Jones, C. H. Nightingale, and M. A. Pfaller. 1997. Antifungal pharmacodynamic characteristics of fluconazole and amphotericin B tested against Candida albicans. Antimicrob Agents Chemother 41:1392-5.

90. Kontoyiannis, D. P., and R. E. Lewis. 2002. Antifungal drug resistance of pathogenic fungi. Lancet 359:1135-44.

91. Krieter, P., B. Flannery, T. Musick, M. Gohdes, M. Martinho, and R. Courtney. 2004. Disposition of posaconazole following single-dose oral administration in healthy subjects. Antimicrob Agents Chemother 48:3543-51.

92. Kuse, E. R., P. Chetchotisakd, C. A. da Cunha, M. Ruhnke, C. Barrios, D. Raghunadharao, J. S. Sekhon, A. Freire, V. Ramasubramanian, I. Demeyer, M. Nucci, A. Leelarasamee, F. Jacobs, J. Decruyenaere, D. Pittet, A. J. Ullmann, L. Ostrosky-Zeichner, O. Lortholary, S. Koblinger, H. DiekmannBerndt, and O. A. Cornely. 2007. Micafungin versus liposomal amphotericin B for candidaemia and invasive candidosis: a phase III randomised double-blind trial. Lancet 369:1519-27.

93. Kvaal, C., S. A. Lachke, T. Srikantha, K. Daniels, J. McCoy, and D. R. Soll. 1999. Misexpression of the opaque-phase-specific gene PEP1 (SAP1) in the white phase of Candida albicans confers increased virulence in a mouse model of cutaneous infection. Infect Immun 67:6652-62.

94. Kvaal, C. A., T. Srikantha, and D. R. Soll. 1997. Misexpression of the whitephase-specific gene WH11 in the opaque phase of Candida albicans affects switching and virulence. Infect Immun 65:4468-75.

95. Lamb, D. C., L. Lei, A. G. Warrilow, G. I. Lepesheva, J. G. Mullins, M. R. Waterman, and S. L. Kelly. 2009. The first virally encoded cytochrome p450. J Virol 83:8266-9.

96. Lenardon, M. D., and A. Nantel. 2012. Rapid detection of aneuploidy following the generation of mutants in Candida albicans. Methods Mol Biol 845:41-9.

97. Leon, C., S. Ruiz-Santana, P. Saavedra, B. Almirante, J. Nolla-Salas, F. Alvarez-Lerma, J. Garnacho-Montero, and M. A. Leon. 2006. A bedside scoring system ("Candida score") for early antifungal treatment in nonneutropenic critically ill patients with Candida colonization. Crit Care Med 34:730-7.

98. Lepesheva, G. I., and M. R. Waterman. 2004. CYP51--the omnipotent P450. Mol Cell Endocrinol 215:165-70. 
99. Li, F., L. Wu, B. Cao, Y. Zhang, X. Li, and Y. Liu. 2013. Surveillance of the prevalence, antibiotic susceptibility, and genotypic characterization of invasive candidiasis in a teaching hospital in China between 2006 to 2011. BMC Infect Dis 13:353.

100. Lin, H. X., and K. A. White. 2004. A complex network of RNA-RNA interactions controls subgenomic mRNA transcription in a tombusvirus. EMBO J 23:3365-74.

101. Lo, H. J., J. R. Kohler, B. DiDomenico, D. Loebenberg, A. Cacciapuoti, and G. R. Fink. 1997. Nonfilamentous C. albicans mutants are avirulent. Cell 90:93949.

102. Lohr, D., P. Venkov, and J. Zlatanova. 1995. Transcriptional regulation in the yeast GAL gene family: a complex genetic network. FASEB J 9:777-87.

103. Lopes da Rosa, J., and P. D. Kaufman. 2012. Chromatin-mediated Candida albicans virulence. Biochim Biophys Acta 1819:349-55.

104. Lopez-Ribot, J. L. 2005. Candida albicans biofilms: more than filamentation. Curr Biol 15:R453-5.

105. Lopez-Ribot, J. L., R. K. McAtee, L. N. Lee, W. R. Kirkpatrick, T. C. White, D. Sanglard, and T. F. Patterson. 1998. Distinct patterns of gene expression associated with development of fluconazole resistance in serial candida albicans isolates from human immunodeficiency virus-infected patients with oropharyngeal candidiasis. Antimicrob Agents Chemother 42:2932-7.

106. Lorenz, M. C., J. A. Bender, and G. R. Fink. 2004. Transcriptional response of Candida albicans upon internalization by macrophages. Eukaryot Cell 3:1076-87.

107. Lutsar, I., S. Roffey, and P. Troke. 2003. Voriconazole concentrations in the cerebrospinal fluid and brain tissue of guinea pigs and immunocompromised patients. Clin Infect Dis 37:728-32.

108. MacPherson, S., B. Akache, S. Weber, X. De Deken, M. Raymond, and B. Turcotte. 2005. Candida albicans zinc cluster protein Upc2p confers resistance to antifungal drugs and is an activator of ergosterol biosynthetic genes. Antimicrob Agents Chemother 49:1745-52.

109. MacPherson, S., M. Larochelle, and B. Turcotte. 2006. A fungal family of transcriptional regulators: the zinc cluster proteins. Microbiol Mol Biol Rev 70:583-604.

110. Manavathu, E. K., J. L. Cutright, and P. H. Chandrasekar. 1998. Organismdependent fungicidal activities of azoles. Antimicrob Agents Chemother 42:301821.

111. Manavathu, E. K., S. Kallakuri, M. T. Arganoza, and J. A. Vazquez. 1999. Amino acid variations of cytochrome P-450 lanosterol 14 alpha-demethylase (CYP51A1) from fluconazoleresistant clinical isolates of Candida albicans. Rev Iberoam Micol 16:198-203.

112. Manoharlal, R., N. A. Gaur, S. L. Panwar, J. Morschhauser, and R. Prasad. 2008. Transcriptional activation and increased mRNA stability contribute to overexpression of CDR1 in azole-resistant Candida albicans. Antimicrob Agents Chemother 52:1481-92. 
113. Mansfield, B. E., H. N. Oltean, B. G. Oliver, S. J. Hoot, S. E. Leyde, L. Hedstrom, and T. C. White. 2010. Azole drugs are imported by facilitated diffusion in Candida albicans and other pathogenic fungi. PLoS Pathog 6:e1001126.

114. Marichal, P., L. Koymans, S. Willemsens, D. Bellens, P. Verhasselt, W. Luyten, M. Borgers, F. C. Ramaekers, F. C. Odds, and H. V. Bossche. 1999. Contribution of mutations in the cytochrome P450 14alpha-demethylase (Erg11p, Cyp51p) to azole resistance in Candida albicans. Microbiology 145 ( Pt 10):270113.

115. Marie, C., S. Leyde, and T. C. White. 2008. Cytoplasmic localization of sterol transcription factors Upc2p and Ecm $22 p$ in S. cerevisiae. Fungal Genet Biol 45:1430-8.

116. Martchenko, M., A. M. Alarco, D. Harcus, and M. Whiteway. 2004. Superoxide dismutases in Candida albicans: transcriptional regulation and functional characterization of the hyphal-induced SOD5 gene. Mol Biol Cell 15:456-67.

117. Mayer, F. L., D. Wilson, and B. Hube. 2013. Candida albicans pathogenicity mechanisms. Virulence 4:119-28.

118. McNeil, M. M., S. L. Nash, R. A. Hajjeh, M. A. Phelan, L. A. Conn, B. D. Plikaytis, and D. W. Warnock. 2001. Trends in mortality due to invasive mycotic diseases in the United States, 1980-1997. Clin Infect Dis 33:641-7.

119. Miyazaki, Y., A. Geber, H. Miyazaki, D. Falconer, T. Parkinson, C. Hitchcock, B. Grimberg, K. Nyswaner, and J. E. Bennett. 1999. Cloning, sequencing, expression and allelic sequence diversity of ERG3 (C-5 sterol desaturase gene) in Candida albicans. Gene 236:43-51.

120. Mogavero, S., A. Tavanti, S. Senesi, P. D. Rogers, and J. Morschhauser. 2011. Differential requirement of the transcription factor Mcm1 for activation of the Candida albicans multidrug efflux pump MDR1 by its regulators Mrr1 and Cap1. Antimicrob Agents Chemother 55:2061-6.

121. Mora-Duarte, J., R. Betts, C. Rotstein, A. L. Colombo, L. Thompson-Moya, J. Smietana, R. Lupinacci, C. Sable, N. Kartsonis, and J. Perfect. 2002. Comparison of caspofungin and amphotericin B for invasive candidiasis. N Engl J Med 347:2020-9.

122. Morio, F., C. Loge, B. Besse, C. Hennequin, and P. Le Pape. 2010. Screening for amino acid substitutions in the Candida albicans Erg11 protein of azolesusceptible and azole-resistant clinical isolates: new substitutions and a review of the literature. Diagn Microbiol Infect Dis 66:373-84.

123. Morio, F., F. Pagniez, C. Lacroix, M. Miegeville, and P. Le Pape. 2012. Amino acid substitutions in the Candida albicans sterol Delta5,6-desaturase (Erg3p) confer azole resistance: characterization of two novel mutants with impaired virulence. J Antimicrob Chemother 67:2131-8.

124. Morrow, C. A., and J. A. Fraser. 2013. Ploidy variation as an adaptive mechanism in human pathogenic fungi. Semin Cell Dev Biol 24:339-46.

125. Morschhauser, J. 2002. The genetic basis of fluconazole resistance development in Candida albicans. Biochim Biophys Acta 1587:240-8. 
126. Morschhauser, J. Regulation of white-opaque switching in Candida albicans. Med Microbiol Immunol 199:165-72.

127. Morschhauser, J., K. S. Barker, T. T. Liu, B. W. J. Bla, R. Homayouni, and P. D. Rogers. 2007. The transcription factor Mrr1p controls expression of the MDR1 efflux pump and mediates multidrug resistance in Candida albicans. PLoS Pathog 3:e164.

128. Muratani, M., C. Kung, K. M. Shokat, and W. P. Tansey. 2005. The F box protein Dsg1/Mdm30 is a transcriptional coactivator that stimulates Gal4 turnover and cotranscriptional mRNA processing. Cell 120:887-99.

129. Naglik, J. R., D. L. Moyes, B. Wachtler, and B. Hube. Candida albicans interactions with epithelial cells and mucosal immunity. Microbes Infect 13:96376.

130. Nelson, D. R. 1999. Cytochrome P450 and the individuality of species. Arch Biochem Biophys 369:1-10.

131. Niwa, T., T. Shiraga, and A. Takagi. 2005. Effect of antifungal drugs on cytochrome P450 (CYP) 2C9, CYP2C19, and CYP3A4 activities in human liver microsomes. Biol Pharm Bull 28:1805-8.

132. Noble, S. M., S. French, L. A. Kohn, V. Chen, and A. D. Johnson. Systematic screens of a Candida albicans homozygous deletion library decouple morphogenetic switching and pathogenicity. Nat Genet 42:590-8.

133. Odds, F. C., M. E. Bougnoux, D. J. Shaw, J. M. Bain, A. D. Davidson, D. Diogo, M. D. Jacobsen, M. Lecomte, S. Y. Li, A. Tavanti, M. C. Maiden, N. A. Gow, and C. d'Enfert. 2007. Molecular phylogenetics of Candida albicans. Eukaryot Cell 6:1041-52.

134. Odds, F. C., A. D. Davidson, M. D. Jacobsen, A. Tavanti, J. A. Whyte, C. C. Kibbler, D. H. Ellis, M. C. Maiden, D. J. Shaw, and N. A. Gow. 2006. Candida albicans strain maintenance, replacement, and microvariation demonstrated by multilocus sequence typing. J Clin Microbiol 44:3647-58.

135. Oliver, B. G., J. L. Song, J. H. Choiniere, and T. C. White. 2007. cis-Acting elements within the Candida albicans ERG11 promoter mediate the azole response through transcription factor Upc2p. Eukaryot Cell 6:2231-9.

136. Orozco, A. S., L. M. Higginbotham, C. A. Hitchcock, T. Parkinson, D. Falconer, A. S. Ibrahim, M. A. Ghannoum, and S. G. Filler. 1998. Mechanism of fluconazole resistance in Candida krusei. Antimicrob Agents Chemother 42:2645-9.

137. Ostrosky-Zeichner, L., J. H. Rex, P. G. Pappas, R. J. Hamill, R. A. Larsen, H. W. Horowitz, W. G. Powderly, N. Hyslop, C. A. Kauffman, J. Cleary, J. E. Mangino, and J. Lee. 2003. Antifungal susceptibility survey of 2,000 bloodstream Candida isolates in the United States. Antimicrob Agents Chemother 47:3149-54.

138. Oude Lashof, A. M., J. D. Sobel, M. Ruhnke, P. G. Pappas, C. Viscoli, H. T. Schlamm, J. H. Rex, and B. J. Kullberg. 2012. Safety and tolerability of voriconazole in patients with baseline renal insufficiency and candidemia. Antimicrob Agents Chemother 56:3133-7. 
139. Pappas, P. G., C. A. Kauffman, D. Andes, D. K. Benjamin, Jr., T. F. Calandra, J. E. Edwards, Jr., S. G. Filler, J. F. Fisher, B. J. Kullberg, L. Ostrosky-Zeichner, A. C. Reboli, J. H. Rex, T. J. Walsh, and J. D. Sobel. 2009. Clinical practice guidelines for the management of candidiasis: 2009 update by the Infectious Diseases Society of America. Clin Infect Dis 48:503-35.

140. Patel, P. K., J. E. Erlandsen, W. R. Kirkpatrick, D. K. Berg, S. D. Westbrook, C. Louden, J. E. Cornell, G. R. Thompson, A. C. Vallor, B. L. Wickes, N. P. Wiederhold, S. W. Redding, and T. F. Patterson. 2012. The Changing Epidemiology of Oropharyngeal Candidiasis in Patients with HIV/AIDS in the Era of Antiretroviral Therapy. AIDS Res Treat 2012:262471.

141. Peng, G., and J. E. Hopper. 2002. Gene activation by interaction of an inhibitor with a cytoplasmic signaling protein. Proc Natl Acad Sci U S A 99:8548-53.

142. Perea, S., J. L. Lopez-Ribot, W. R. Kirkpatrick, R. K. McAtee, R. A. Santillan, M. Martinez, D. Calabrese, D. Sanglard, and T. F. Patterson. 2001. Prevalence of molecular mechanisms of resistance to azole antifungal agents in Candida albicans strains displaying high-level fluconazole resistance isolated from human immunodeficiency virus-infected patients. Antimicrob Agents Chemother 45:2676-84.

143. Pfaller, M., D. Neofytos, D. Diekema, N. Azie, H. U. Meier-Kriesche, S. P. Quan, and D. Horn. 2012. Epidemiology and outcomes of candidemia in 3648 patients: data from the Prospective Antifungal Therapy (PATH Alliance(R)) registry, 2004-2008. Diagn Microbiol Infect Dis 74:323-31.

144. Pfaller, M. A. 2012. Antifungal drug resistance: mechanisms, epidemiology, and consequences for treatment. Am J Med 125:S3-13.

145. Pfaller, M. A., D. Andes, D. J. Diekema, A. Espinel-Ingroff, and D. Sheehan. 2010. Wild-type MIC distributions, epidemiological cutoff values and speciesspecific clinical breakpoints for fluconazole and Candida: time for harmonization of CLSI and EUCAST broth microdilution methods. Drug Resist Updat 13:18095.

146. Pfaller, M. A., L. Boyken, R. J. Hollis, S. A. Messer, S. Tendolkar, and D. J. Diekema. 2005. In vitro activities of anidulafungin against more than 2,500 clinical isolates of Candida spp., including 315 isolates resistant to fluconazole. J Clin Microbiol 43:5425-7.

147. Pfaller, M. A., D. J. Diekema, S. A. Messer, R. J. Hollis, and R. N. Jones. 2003. In vitro activities of caspofungin compared with those of fluconazole and itraconazole against 3,959 clinical isolates of Candida spp., including 157 fluconazole-resistant isolates. Antimicrob Agents Chemother 47:1068-71.

148. Pfaller, M. A., S. A. Messer, L. Boyken, R. J. Hollis, C. Rice, S. Tendolkar, and D. J. Diekema. 2004. In vitro activities of voriconazole, posaconazole, and fluconazole against 4,169 clinical isolates of Candida spp. and Cryptococcus neoformans collected during 2001 and 2002 in the ARTEMIS global antifungal surveillance program. Diagn Microbiol Infect Dis 48:201-5.

149. Pfaller, M. A., S. A. Messer, R. J. Hollis, and R. N. Jones. 2001. In vitro activities of posaconazole (Sch 56592) compared with those of itraconazole and fluconazole against 3,685 clinical isolates of Candida spp. and Cryptococcus neoformans. Antimicrob Agents Chemother 45:2862-4. 
150. Pittet, D., M. Monod, P. M. Suter, E. Frenk, and R. Auckenthaler. 1994. Candida colonization and subsequent infections in critically ill surgical patients. Ann Surg 220:751-8.

151. Poirier, J. M., and G. Cheymol. 1998. Optimisation of itraconazole therapy using target drug concentrations. Clin Pharmacokinet 35:461-73.

152. Prasad, R., P. De Wergifosse, A. Goffeau, and E. Balzi. 1995. Molecular cloning and characterization of a novel gene of Candida albicans, CDR1, conferring multiple resistance to drugs and antifungals. Curr Genet 27:320-9.

153. Rangel-Frausto, M. S., T. Wiblin, H. M. Blumberg, L. Saiman, J. Patterson, M. Rinaldi, M. Pfaller, J. E. Edwards, Jr., W. Jarvis, J. Dawson, and R. P. Wenzel. 1999. National epidemiology of mycoses survey (NEMIS): variations in rates of bloodstream infections due to Candida species in seven surgical intensive care units and six neonatal intensive care units. Clin Infect Dis 29:253-8.

154. Reboli, A. C., C. Rotstein, P. G. Pappas, S. W. Chapman, D. H. Kett, D. Kumar, R. Betts, M. Wible, B. P. Goldstein, J. Schranz, D. S. Krause, and T. J. Walsh. 2007. Anidulafungin versus fluconazole for invasive candidiasis. N Engl J Med 356:2472-82.

155. Redding, S., J. Smith, G. Farinacci, M. Rinaldi, A. Fothergill, J. RhineChalberg, and M. Pfaller. 1994. Resistance of Candida albicans to fluconazole during treatment of oropharyngeal candidiasis in a patient with AIDS: documentation by in vitro susceptibility testing and DNA subtype analysis. Clin Infect Dis 18:240-2.

156. Reuss, O., A. Vik, R. Kolter, and J. Morschhauser. 2004. The SAT1 flipper, an optimized tool for gene disruption in Candida albicans. Gene 341:119-27.

157. Rex, J. H., M. G. Rinaldi, and M. A. Pfaller. 1995. Resistance of Candida species to fluconazole. Antimicrob Agents Chemother 39:1-8.

158. Rogers, P. D., and K. S. Barker. 2002. Evaluation of differential gene expression in fluconazole-susceptible and -resistant isolates of Candida albicans by cDNA microarray analysis. Antimicrob Agents Chemother 46:3412-7.

159. Saag, M. S., R. J. Graybill, R. A. Larsen, P. G. Pappas, J. R. Perfect, W. G. Powderly, J. D. Sobel, and W. E. Dismukes. 2000. Practice guidelines for the management of cryptococcal disease. Infectious Diseases Society of America. Clin Infect Dis 30:710-8.

160. Sadowski, I., C. Costa, and R. Dhanawansa. 1996. Phosphorylation of Ga14p at a single $\mathrm{C}$-terminal residue is necessary for galactose-inducible transcription. Mol Cell Biol 16:4879-87.

161. Salmeron, J. M., Jr., K. K. Leuther, and S. A. Johnston. 1990. GAL4 mutations that separate the transcriptional activation and GAL80-interactive functions of the yeast GAL4 protein. Genetics 125:21-7.

162. Sanglard, D., F. Ischer, L. Koymans, and J. Bille. 1998. Amino acid substitutions in the cytochrome P-450 lanosterol 14alpha-demethylase (CYP51A1) from azole-resistant Candida albicans clinical isolates contribute to resistance to azole antifungal agents. Antimicrob Agents Chemother 42:241-53.

163. Sanglard, D., F. Ischer, M. Monod, and J. Bille. 1997. Cloning of Candida albicans genes conferring resistance to azole antifungal agents: characterization of CDR2, a new multidrug ABC transporter gene. Microbiology 143 ( Pt 2):405-16. 
164. Sanglard, D., F. Ischer, M. Monod, and J. Bille. 1996. Susceptibilities of Candida albicans multidrug transporter mutants to various antifungal agents and other metabolic inhibitors. Antimicrob Agents Chemother 40:2300-5.

165. Sanglard, D., K. Kuchler, F. Ischer, J. L. Pagani, M. Monod, and J. Bille. 1995. Mechanisms of resistance to azole antifungal agents in Candida albicans isolates from AIDS patients involve specific multidrug transporters. Antimicrob Agents Chemother 39:2378-86.

166. Sasse, C., N. Dunkel, T. Schafer, S. Schneider, F. Dierolf, K. Ohlsen, and J. Morschhauser. 2012. The stepwise acquisition of fluconazole resistance mutations causes a gradual loss of fitness in Candida albicans. Mol Microbiol 86:539-56.

167. Sasse, C., M. Hasenberg, M. Weyler, M. Gunzer, and J. Morschhauser. 2013. White-opaque switching of Candida albicans allows immune evasion in an environment-dependent fashion. Eukaryot Cell 12:50-8.

168. Schillig, R., and J. Morschhauser. 2013. Analysis of a fungus-specific transcription factor family, the Candida albicans zinc cluster proteins, by artificial activation. Mol Microbiol.

169. Schubert, S., K. S. Barker, S. Znaidi, S. Schneider, F. Dierolf, N. Dunkel, M. Aid, G. Boucher, P. D. Rogers, M. Raymond, and J. Morschhauser. 2011. Regulation of efflux pump expression and drug resistance by the transcription factors Mrr1, Upc2, and Cap1 in Candida albicans. Antimicrob Agents Chemother 55:2212-23.

170. Sellam, A., C. Askew, E. Epp, F. Tebbji, A. Mullick, M. Whiteway, and A. Nantel. 2010. Role of transcription factor CaNdt80p in cell separation, hyphal growth, and virulence in Candida albicans. Eukaryot Cell 9:634-44.

171. Sellam, A., F. Tebbji, and A. Nantel. 2009. Role of Ndt80p in sterol metabolism regulation and azole resistance in Candida albicans. Eukaryot Cell 8:1174-83.

172. Selmecki, A., S. Bergmann, and J. Berman. 2005. Comparative genome hybridization reveals widespread aneuploidy in Candida albicans laboratory strains. Mol Microbiol 55:1553-65.

173. Selmecki, A., A. Forche, and J. Berman. 2006. Aneuploidy and isochromosome formation in drug-resistant Candida albicans. Science 313:367-70.

174. Selmecki, A. M., K. Dulmage, L. E. Cowen, J. B. Anderson, and J. Berman. 2009. Acquisition of aneuploidy provides increased fitness during the evolution of antifungal drug resistance. PLoS Genet 5:e1000705.

175. Shapiro, R. S., N. Robbins, and L. E. Cowen. 2011. Regulatory circuitry governing fungal development, drug resistance, and disease. Microbiol Mol Biol Rev 75:213-67.

176. Shields, R. K., M. H. Nguyen, E. G. Press, C. L. Updike, and C. J. Clancy. 2013. Caspofungin MICs correlate with treatment outcomes among patients with Candida glabrata invasive candidiasis and prior echinocandin exposure. Antimicrob Agents Chemother 57:3528-35.

177. Silver, P. M., B. G. Oliver, and T. C. White. 2004. Role of Candida albicans transcription factor Upc2p in drug resistance and sterol metabolism. Eukaryot Cell 3:1391-7. 
178. Singh-Babak, S. D., T. Babak, S. Diezmann, J. A. Hill, J. L. Xie, Y. L. Chen, S. M. Poutanen, R. P. Rennie, J. Heitman, and L. E. Cowen. Global analysis of the evolution and mechanism of echinocandin resistance in Candida glabrata. PLoS Pathog 8:e1002718.

179. Soll, D. R., and C. Pujol. 2003. Candida albicans clades. FEMS Immunol Med Microbiol 39:1-7.

180. Soustre, J., M. H. Rodier, S. Imbert-Bouyer, G. Daniault, and C. Imbert. 2004. Caspofungin modulates in vitro adherence of Candida albicans to plastic coated with extracellular matrix proteins. J Antimicrob Chemother 53:522-5.

181. Spinillo, A., E. Capuzzo, R. Gulminetti, P. Marone, L. Colonna, and G. Piazzi. 1997. Prevalence of and risk factors for fungal vaginitis caused by nonalbicans species. Am J Obstet Gynecol 176:138-41.

182. Staab, J. F., and P. Sundstrom. 2003. URA3 as a selectable marker for disruption and virulence assessment of Candida albicans genes. Trends Microbiol 11:69-73.

183. Sugita, T., and T. Nakase. 1999. Non-universal usage of the leucine CUG codon and the molecular phylogeny of the genus Candida. Syst Appl Microbiol 22:7986.

184. Sun, J. N., N. V. Solis, Q. T. Phan, J. S. Bajwa, H. Kashleva, A. Thompson, Y. Liu, A. Dongari-Bagtzoglou, M. Edgerton, and S. G. Filler. Host cell invasion and virulence mediated by Candida albicans Ssa1. PLoS Pathog 6:e1001181.

185. Talibi, D., and M. Raymond. 1999. Isolation of a putative Candida albicans transcriptional regulator involved in pleiotropic drug resistance by functional complementation of a pdr1 pdr3 mutation in Saccharomyces cerevisiae. J Bacteriol 181:231-40.

186. Templeton, I. E., K. E. Thummel, E. D. Kharasch, K. L. Kunze, C. Hoffer, W. L. Nelson, and N. Isoherranen. 2008. Contribution of itraconazole metabolites to inhibition of CYP3A4 in vivo. Clin Pharmacol Ther 83:77-85.

187. Thaler, F., B. Bernard, M. Tod, C. P. Jedynak, O. Petitjean, P. Derome, and P. Loirat. 1995. Fluconazole penetration in cerebral parenchyma in humans at steady state. Antimicrob Agents Chemother 39:1154-6.

188. Thompson, G. R., 3rd, P. K. Patel, W. R. Kirkpatrick, S. D. Westbrook, D. Berg, J. Erlandsen, S. W. Redding, and T. F. Patterson. 2010. Oropharyngeal candidiasis in the era of antiretroviral therapy. Oral Surg Oral Med Oral Pathol Oral Radiol Endod 109:488-95.

189. Tsao, S., F. Rahkhoodaee, and M. Raymond. 2009. Relative contributions of the Candida albicans ABC transporters Cdr1p and Cdr2p to clinical azole resistance. Antimicrob Agents Chemother 53:1344-52.

190. Uchaipichat, V., L. K. Winner, P. I. Mackenzie, D. J. Elliot, J. A. Williams, and J. O. Miners. 2006. Quantitative prediction of in vivo inhibitory interactions involving glucuronidated drugs from in vitro data: the effect of fluconazole on zidovudine glucuronidation. Br J Clin Pharmacol 61:427-39. 
191. Uppuluri, P., A. K. Chaturvedi, A. Srinivasan, M. Banerjee, A. K. Ramasubramaniam, J. R. Kohler, D. Kadosh, and J. L. Lopez-Ribot. Dispersion as an important step in the Candida albicans biofilm developmental cycle. PLoS Pathog 6:e1000828.

192. Vajda, V., and S. McLoughlin. 2004. Fungal proliferation at the CretaceousTertiary boundary. Science 303:1489.

193. Vale-Silva, L. A., A. T. Coste, F. Ischer, J. E. Parker, S. L. Kelly, E. Pinto, and D. Sanglard. 2012. Azole resistance by loss of function of the sterol Delta(5),(6)-desaturase gene (ERG3) in Candida albicans does not necessarily decrease virulence. Antimicrob Agents Chemother 56:1960-8.

194. Vermes, A., H. J. Guchelaar, and J. Dankert. 2000. Flucytosine: a review of its pharmacology, clinical indications, pharmacokinetics, toxicity and drug interactions. J Antimicrob Chemother 46:171-9.

195. Vermitsky, J. P., M. J. Self, S. G. Chadwick, J. P. Trama, M. E. Adelson, E. Mordechai, and S. E. Gygax. 2008. Survey of vaginal-flora Candida species isolates from women of different age groups by use of species-specific PCR detection. J Clin Microbiol 46:1501-3.

196. Viale, P. 2009. Candida colonization and candiduria in critically ill patients in the intensive care unit. Drugs 69 Suppl 1:51-7.

197. Vik, A., and J. Rine. 2001. Upc2p and Ecm22p, dual regulators of sterol biosynthesis in Saccharomyces cerevisiae. Mol Cell Biol 21:6395-405.

198. Vincent, J. L., J. Rello, J. Marshall, E. Silva, A. Anzueto, C. D. Martin, R. Moreno, J. Lipman, C. Gomersall, Y. Sakr, and K. Reinhart. 2009. International study of the prevalence and outcomes of infection in intensive care units. JAMA 302:2323-9.

199. Wang, J. S., Y. L. Yang, C. J. Wu, K. J. Ouyang, K. Y. Tseng, C. G. Chen, H. Wang, and H. J. Lo. 2006. The DNA-binding domain of CaNdt80p is required to activate CDR1 involved in drug resistance in Candida albicans. J Med Microbiol 55:1403-11.

200. Warrilow, A. G., C. M. Martel, J. E. Parker, N. Melo, D. C. Lamb, W. D. Nes, D. E. Kelly, and S. L. Kelly. 2010. Azole binding properties of Candida albicans sterol 14-alpha demethylase (CaCYP51). Antimicrob Agents Chemother 54:4235-45.

201. Warrilow, A. G., J. G. Mullins, C. M. Hull, J. E. Parker, D. C. Lamb, D. E. Kelly, and S. L. Kelly. 2012. S279 point mutations in Candida albicans Sterol 14-alpha demethylase (CYP51) reduce in vitro inhibition by fluconazole. Antimicrob Agents Chemother 56:2099-107.

202. Weiss, J., M. M. Ten Hoevel, J. Burhenne, I. Walter-Sack, M. M. Hoffmann, J. Rengelshausen, W. E. Haefeli, and G. Mikus. 2009. CYP2C19 genotype is a major factor contributing to the highly variable pharmacokinetics of voriconazole. J Clin Pharmacol 49:196-204.

203. White, T. C., K. A. Marr, and R. A. Bowden. 1998. Clinical, cellular, and molecular factors that contribute to antifungal drug resistance. Clin Microbiol Rev 11:382-402. 
204. Wille, M. P., T. Guimaraes, G. H. Furtado, and A. L. Colombo. 2013. Historical trends in the epidemiology of candidaemia: analysis of an 11-year period in a tertiary care hospital in Brazil. Mem Inst Oswaldo Cruz 108.

205. Wisplinghoff, H., T. Bischoff, S. M. Tallent, H. Seifert, R. P. Wenzel, and M. B. Edmond. 2004. Nosocomial bloodstream infections in US hospitals: analysis of 24,179 cases from a prospective nationwide surveillance study. Clin Infect Dis 39:309-17.

206. Wu, Y., R. J. Reece, and M. Ptashne. 1996. Quantitation of putative activatortarget affinities predicts transcriptional activating potentials. EMBO J 15:395163.

207. Wysong, D. R., L. Christin, A. M. Sugar, P. W. Robbins, and R. D. Diamond. 1998. Cloning and sequencing of a Candida albicans catalase gene and effects of disruption of this gene. Infect Immun 66:1953-61.

208. Xiao, L., V. Madison, A. S. Chau, D. Loebenberg, R. E. Palermo, and P. M. McNicholas. 2004. Three-dimensional models of wild-type and mutated forms of cytochrome P450 14alpha-sterol demethylases from Aspergillus fumigatus and Candida albicans provide insights into posaconazole binding. Antimicrob Agents Chemother 48:568-74.

209. Yoshida, Y. 1988. Cytochrome P450 of fungi: primary target for azole antifungal agents. Curr Top Med Mycol 2:388-418.

210. Yoshida, Y., Y. Aoyama, M. Noshiro, and O. Gotoh. 2000. Sterol 14demethylase P450 (CYP51) provides a breakthrough for the discussion on the evolution of cytochrome P450 gene superfamily. Biochem Biophys Res Commun 273:799-804.

211. Zaas, A. K., and B. D. Alexander. 2005. Echinocandins: role in antifungal therapy, 2005. Expert Opin Pharmacother 6:1657-68.

212. Zhu, W., and S. G. Filler. Interactions of Candida albicans with epithelial cells. Cell Microbiol 12:273-82.

213. Zimmermann, T., R. A. Yeates, M. Albrecht, H. Laufen, and A. Wildfeuer. 1994. Influence of concomitant food intake on the gastrointestinal absorption of fluconazole and itraconazole in Japanese subjects. Int J Clin Pharmacol Res 14:87-93.

214. Zimmermann, T., R. A. Yeates, H. Laufen, G. Pfaff, and A. Wildfeuer. 1994. Influence of concomitant food intake on the oral absorption of two triazole antifungal agents, itraconazole and fluconazole. Eur J Clin Pharmacol 46:147-50.

215. Zimmermann, T., R. A. Yeates, K. D. Riedel, P. Lach, and H. Laufen. 1994. The influence of gastric $\mathrm{pH}$ on the pharmacokinetics of fluconazole: the effect of omeprazole. Int J Clin Pharmacol Ther 32:491-6.

216. Znaidi, S., X. De Deken, S. Weber, T. Rigby, A. Nantel, and M. Raymond. 2007. The zinc cluster transcription factor Tac1p regulates PDR16 expression in Candida albicans. Mol Microbiol 66:440-52.

217. Znaidi, S., S. Weber, O. Z. Al-Abdin, P. Bomme, S. Saidane, S. Drouin, S. Lemieux, X. De Deken, F. Robert, and M. Raymond. 2008. Genomewide location analysis of Candida albicans Upc2p, a regulator of sterol metabolism and azole drug resistance. Eukaryot Cell 7:836-47. 


\section{APPENDIX A. C. ALBICANS STRAINS TABLE}

\begin{tabular}{|c|c|c|c|c|c|}
\hline Strain \# & Modified strain identification & Source if known & 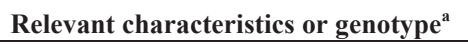 & Source & UPC2 polymorphisms observed (amino acid substitutions) \\
\hline \multicolumn{6}{|c|}{ Clinical isolates } \\
\hline SC5314 & SC5314 & & $U P C 2-1 / U P C 2-2$ & ATCC & \\
\hline 20644.082 & 1 & & $\mathrm{FLU}^{\mathrm{S}}$ isolate & University of Iowa & \\
\hline 20205.043 & 2 & Blood & $\mathrm{FLU}^{\mathrm{S}}$ isolate & University of Iowa & \\
\hline 20339.016 & 3 & Esophogeal & $\mathrm{FLU}^{\mathrm{S}}$ isolate & University of Iowa & G1791A (M597I) \\
\hline 20389.007 & 4 & & $\mathrm{FLU}^{\mathrm{S}}$ isolate & University of Iowa & \\
\hline 20646.057 & 5 & Blood & $\mathrm{FLU}^{\mathrm{S}}$ isolate & University of Iowa & \\
\hline 20313.046 & 6 & Throat & $\mathrm{FLU}^{\mathrm{S}}$ isolate & University of Iowa & \\
\hline 20510.016 & 7 & Abdom.Fluid & $\mathrm{FLU}^{\mathrm{S}}$ isolate & University of Iowa & \\
\hline 20464.007 & 8 & Sput & $\mathrm{FLU}^{\mathrm{S}}$ isolate & University of Iowa & \\
\hline 20646.057 & 9 & Blood & $\mathrm{FLU}^{\mathrm{S}}$ isolate & University of Iowa & \\
\hline 20324.046 & 10 & Blood & $\mathrm{FLU}^{\mathrm{s}}$ isolate & University of Iowa & C449T (P150L); A817G (T273A) \\
\hline 20512.088 & 11 & & $\mathrm{FLU}^{\mathrm{R}}$ isolate & University of Iowa & \\
\hline 20512.089 & 12 & & $\mathrm{FLU}^{\mathrm{R}}$ isolate & University of Iowa & \\
\hline 20533.031 & 13 & & $\mathrm{FLU}^{\mathrm{R}}$ isolate & University of Iowa & G1927A (A643T) \\
\hline 20637.057 & 14 & Blood & $\mathrm{FLU}^{\mathrm{R}}$ isolate & University of Iowa & \\
\hline 20513.024 & 15 & & $\mathrm{FLU}^{\mathrm{R}}$ isolate & University of Iowa & G1434C (W478C) \\
\hline 20288.098 & 16 & & $\mathrm{FLU}^{\mathrm{R}}$ isolate & University of Iowa & \\
\hline 20642.062 & 17 & & $\mathrm{FLU}^{\mathrm{R}}$ isolate & University of Iowa & \\
\hline 20513.009 & 18 & & $\mathrm{FLU}^{\mathrm{R}}$ isolate & University of Iowa & \\
\hline 20513.01 & 19 & & $\mathrm{FLU}^{\mathrm{R}}$ isolate & University of Iowa & G910A (G304R) \\
\hline 20513.011 & 20 & & $\mathrm{FLU}^{\mathrm{R}}$ isolate & University of Iowa & G910A (G304R) \\
\hline
\end{tabular}


Appendix A. Continued.

\begin{tabular}{|c|c|c|c|c|c|}
\hline Strain \# & Modified strain identification & Source if known & Relevant characteristics or genotype $\mathrm{e}^{\mathrm{a}}$ & Source & UPC2 polymorphisms observed (amino acid substitutions) \\
\hline 20513.016 & 21 & & $\mathrm{FLU}^{\mathrm{R}}$ isolate & University of Iowa & G1943A (G648D) \\
\hline 20245.061 & 22 & Abdom.Fluid & $\mathrm{FLU}^{\mathrm{R}}$ isolate & University of Iowa & \\
\hline 20323.066 & 23 & Throat & $\mathrm{FLU}^{\mathrm{R}}$ isolate & University of Iowa & \\
\hline 20339.013 & 24 & Esophogeal & $\mathrm{FLU}^{\mathrm{R}}$ isolate & University of Iowa & \\
\hline 20339.027 & 25 & Oral & $\mathrm{FLU}^{\mathrm{R}}$ isolate & University of Iowa & G1942A (G648S) \\
\hline 20339.063 & 26 & Esophogeal & $\mathrm{FLU}^{\mathrm{R}}$ isolate & University of Iowa & \\
\hline 20513.025 & 27 & & $\mathrm{FLU}^{\mathrm{R}}$ isolate & University of Iowa & G1434C(W478C) \\
\hline 20513.026 & 28 & & $\mathrm{FLU}^{\mathrm{R}}$ isolate & University of Iowa & \\
\hline 20408.049 & 29 & Tissue & $\mathrm{FLU}^{\mathrm{R}}$ isolate & University of Iowa & \\
\hline 20531.041 & 30 & Blood & $\mathrm{FLU}^{\mathrm{R}}$ isolate & University of Iowa & C1928T (A643V) \\
\hline 20628.078 & 31 & & $\mathrm{FLU}^{\mathrm{R}}$ isolate & University of Iowa & \\
\hline 20288.080 & 32 & Tissue & $\mathrm{FLU}^{\mathrm{R}}$ isolate & University of Iowa & \\
\hline 20323.052 & 33 & Throat & $\mathrm{FLU}^{\mathrm{R}}$ isolate & University of Iowa & \\
\hline 20339.014 & 34 & Esophogeal & $\mathrm{FLU}^{\mathrm{R}}$ isolate & University of Iowa & \\
\hline 20339.028 & 35 & Esophogeal & $\mathrm{FLU}^{\mathrm{R}}$ isolate & University of Iowa & G1942A (G648S) \\
\hline 20339.044 & 36 & Oropharynx & $\mathrm{FLU}^{\mathrm{R}}$ isolate & University of Iowa & A1925T (Y642F) \\
\hline 20339.045 & 37 & Buccal & $\mathrm{FLU}^{\mathrm{R}}$ isolate & University of Iowa & A1925T (Y642F) \\
\hline 20339.027 & 38 & Oral & $\mathrm{FLU}^{\mathrm{R}}$ isolate & University of Iowa & G1943A (G648D) \\
\hline 20339.062 & 39 & Esophogeal & $\mathrm{FLU}^{\mathrm{R}}$ isolate & University of Iowa & \\
\hline 20408.059 & 40 & Blood & $\mathrm{FLU}^{\mathrm{R}}$ isolate & University of Iowa & \\
\hline 20348.024 & 41 & Sputum & $\mathrm{FLU}^{\mathrm{R}}$ isolate & University of Iowa & \\
\hline 20512.090 & 42 & & $\mathrm{FLU}^{\mathrm{R}}$ isolate & University of Iowa & \\
\hline 20339.003 & 43 & Buccal & $\mathrm{FLU}^{\mathrm{R}}$ isolate & University of Iowa & \\
\hline 20339.025 & 44 & Oral & $\mathrm{FLU}^{\mathrm{R}}$ isolate & University of Iowa & \\
\hline
\end{tabular}


Appendix A. Continued.

\begin{tabular}{|c|c|c|c|c|c|}
\hline Strain \# & Modified strain identification & Source if known & Relevant characteristics or genotype $\mathrm{e}^{\mathrm{a}}$ & Source & UPC2 polymorphisms observed (amino acid substitutions) \\
\hline 20339.048 & 45 & Esophogeal & $\mathrm{FLU}^{\mathrm{R}}$ isolate & University of Iowa & C1937T (A646V) \\
\hline 20339.064 & 46 & Esophogeal & $\mathrm{FLU}^{\mathrm{R}}$ isolate & University of Iowa & \\
\hline 20339.072 & 47 & Esophogeal & $\mathrm{FLU}^{\mathrm{R}}$ isolate & University of Iowa & G1943A (G648D) \\
\hline 20512.099 & 48 & & $\mathrm{FLU}^{\mathrm{R}}$ isolate & University of Iowa & \\
\hline 20377.041 & 49 & Pleural F1 & $\mathrm{FLU}^{\mathrm{R}}$ isolate & University of Iowa & \\
\hline 20323.080 & 50 & Throat & $\mathrm{FLU}^{\mathrm{R}}$ isolate & University of Iowa & \\
\hline 20339.008 & 51 & Oral & $\mathrm{FLU}^{\mathrm{R}}$ isolate & University of Iowa & \\
\hline 20339.058 & 52 & Esophogeal & $\mathrm{FLU}^{\mathrm{R}}$ isolate & University of Iowa & G1943A (G648D) \\
\hline 20672.028 & 53 & Blood & $\mathrm{FLU}^{\mathrm{R}}$ isolate & University of Iowa & \\
\hline 20495.091 & 54 & Abscess & $\mathrm{FLU}^{\mathrm{R}}$ isolate & University of Iowa & $\begin{array}{c}\mathrm{C} 406 \mathrm{~T} \text { (P136S), } \mathrm{A671G}(\mathrm{N} 224 \mathrm{~S}), \mathrm{A} 749 \mathrm{G}(\mathrm{N} 250 \mathrm{~S}), \mathrm{T} 1952 \mathrm{C} \\
(\mathrm{M} 651 \mathrm{~T})\end{array}$ \\
\hline 20513.014 & 55 & & $\mathrm{FLU}^{\mathrm{R}}$ isolate & University of Iowa & G1942A (G648S) \\
\hline 20513.021 & 56 & & $\mathrm{FLU}^{\mathrm{R}}$ isolate & University of Iowa & G1942A (G648S) \\
\hline 20513.022 & 57 & & $\mathrm{FLU}^{\mathrm{R}}$ isolate & University of Iowa & G1942A (G648S) \\
\hline 20513.023 & 58 & & $\mathrm{FLU}^{\mathrm{R}}$ isolate & University of Iowa & G1942A (G648S) \\
\hline 20430.009 & 59 & Blood & $\mathrm{FLU}^{\mathrm{R}}$ isolate & University of Iowa & G1943A (G648D) \\
\hline 20288.030 & 60 & Tissue & $\mathrm{FLU}^{\mathrm{R}}$ isolate & University of Iowa & \\
\hline 20323.083 & 61 & Throat & $\mathrm{FLU}^{\mathrm{R}}$ isolate & University of Iowa & \\
\hline 20339.006 & 62 & Oral & $\mathrm{FLU}^{\mathrm{R}}$ isolate & University of Iowa & \\
\hline 20339.011 & 63 & Esophogeal & $\mathrm{FLU}^{\mathrm{R}}$ isolate & University of Iowa & \\
\hline 20339.015 & 64 & Oral & $\mathrm{FLU}^{\mathrm{R}}$ isolate & University of Iowa & \\
\hline 20339.020 & 65 & Oropharynx & $\mathrm{FLU}^{\mathrm{R}}$ isolate & University of Iowa & G1942A (G648S) \\
\hline 20339.021 & 66 & Oropharynx & $\mathrm{FLU}^{\mathrm{R}}$ isolate & University of Iowa & \\
\hline 20339.022 & 67 & Oropharynx & $\mathrm{FLU}^{\mathrm{R}}$ isolate & University of Iowa & G1942A (G648S) \\
\hline
\end{tabular}


Appendix A. Continued.

\begin{tabular}{|c|c|c|c|c|c|}
\hline Strain \# & Modified strain identification & Source if known & Relevant characteristics or genotype $\mathrm{e}^{\mathrm{a}}$ & Source & UPC2 polymorphisms observed (amino acid substitutions) \\
\hline 20339.030 & 68 & Oropharynx & $\mathrm{FLU}^{\mathrm{R}}$ isolate & University of Iowa & A817G (T273A), C1928T (A643V) \\
\hline 20339.032 & 69 & Oropharynx & $\mathrm{FLU}^{\mathrm{R}}$ isolate & University of Iowa & G1942A (G648S) \\
\hline 20339.049 & 70 & Esophogeal & $\mathrm{FLU}^{\mathrm{R}}$ isolate & University of Iowa & C1937T (A646V) \\
\hline 20339.070 & 71 & Oral & $\mathrm{FLU}^{\mathrm{R}}$ isolate & University of Iowa & G1943A (G648D) \\
\hline 20513.02 & 72 & & $\mathrm{FLU}^{\mathrm{R}}$ isolate & University of Iowa & \\
\hline
\end{tabular}

$\mathrm{FLU}^{\mathrm{S}}$; fluconazole susceptible. $\mathrm{FLU}^{\mathrm{R}}$; fluconazole resistant 
APPENDIX B. GENES UPREGULATED IN STRAINS CARRYING UPC2 GOF ALLELES BY AT LEAST 1.5 FOLD

\begin{tabular}{|c|c|c|c|c|c|c|c|c|c|c|}
\hline \multirow[b]{3}{*}{ CGD Name } & \multirow[b]{3}{*}{ orf19 Designation } & \multicolumn{8}{|c|}{ Change in fold expression ${ }^{a}$} & \multirow[b]{3}{*}{ Molecular function } \\
\hline & & \multicolumn{2}{|c|}{$\underline{\text { G648S }}$} & \multicolumn{2}{|c|}{ G648D } & \multicolumn{2}{|c|}{$\underline{Y 642 F}$} & \multicolumn{2}{|c|}{$\underline{\mathrm{A} 643 \mathrm{~V}}$} & \\
\hline & & Exp1 & Exp2 & Exp1 & Exp2 & Exp1 & Exp2 & Exp1 & Exp2 & \\
\hline$A N P 1$ & orf19.3622 & 1.9 & 1.5 & 1.5 & 1.2 & 1.3 & 1.4 & 1.1 & 1.0 & contributes_to alpha-1,6-mannosyltransferase activity (IEA) \\
\hline FRE9 & orf19.3538 & 2.1 & 1.5 & 1.2 & 1.4 & 1.0 & 1.3 & 1.2 & 1.7 & ferric-chelate reductase activity (IEA) \\
\hline GST1 & orf19.3121 & 7.8 & 2.0 & 38.0 & 1.2 & 16.4 & 0.9 & 13.5 & 0.9 & transcription corepressor activity (IEA) \\
\hline IFCl & orf19.3746 & 1.9 & 1.8 & 0.9 & 0.9 & 1.3 & 0.7 & 1.2 & 0.8 & oligopeptide transporter activity (IGI, ISS) \\
\hline LTE1 & orf19.2238 & 18.2 & 2.0 & 9.8 & 0.5 & 19.1 & 1.3 & 10.1 & 0.8 & guanyl-nucleotide exchange factor activity (IEA) \\
\hline NBP35 & orf19.747 & 1.8 & 1.5 & 2.1 & 1.4 & 1.6 & 1.0 & 2.1 & 1.2 & nucleotide binding (NAS) $\mid 4$ iron, 4 sulfur cluster binding (IEA) \\
\hline PHO114 & orf19.4584 & 1.7 & 1.6 & 2.2 & 1.4 & 1.8 & 0.9 & 2.0 & 1.3 & acid phosphatase activity (IEA) \\
\hline$P L B 1$ & orf19.689 & 1.6 & 2.0 & 1.9 & 1.2 & 1.3 & 1.1 & 1.4 & 1.1 & lysophospholipase activity (IDA, IMP, ISS) \\
\hline PLB4 & orf19.1443 & 1.6 & 1.5 & 2.0 & 1.3 & 1.4 & 1.3 & 1.5 & 1.3 & lysophospholipase activity (ISS) \\
\hline PRR2 & orf19.1341 & 1.5 & 2.5 & 2.9 & 1.1 & 0.9 & 0.9 & 1.1 & 1.6 & receptor signaling protein serine/threonine kinase activity (IEA) \\
\hline$R B T 1$ & orf19.1327 & 1.9 & 1.6 & 1.2 & 1.1 & 1.6 & 1.9 & 1.1 & 1.2 & \\
\hline$S M C 3$ & orf19.262 & 1.5 & 1.7 & 1.3 & 0.9 & 1.8 & 0.8 & 1.4 & 0.7 & ATPase activity (IEA) \\
\hline SUA72 & orf19.3519 & 1.5 & 1.7 & 2.3 & 0.8 & 1.3 & 1.0 & 1.7 & 1.2 & general RNA polymerase II transcription factor activity (IEA) \\
\hline \multirow[t]{10}{*}{$Z R T 1$} & orf19.3112 & 1.5 & 1.6 & 1.6 & 1.4 & 1.1 & 0.8 & 1.2 & 1.0 & low-affinity zinc ion transmembrane transporter activity (IEA) \\
\hline & orf19.11618 & 1.5 & 1.6 & 1.6 & 0.7 & 0.9 & 1.0 & 1.8 & 0.8 & \\
\hline & orf19.1768 & 1.6 & 1.7 & 1.3 & 1.0 & 1.6 & 1.1 & 1.6 & 0.9 & \\
\hline & orf19.302 & 1.7 & 1.5 & 1.3 & 1.1 & 1.0 & 0.7 & 1.4 & 1.2 & contributes_to Rab guanyl-nucleotide exchange factor activity (IEA) \\
\hline & orf19.3156 & 2.1 & 1.5 & 1.9 & 1.0 & 1.7 & 1.0 & 1.5 & 1.0 & \\
\hline & orf19.3813 & 1.5 & 1.8 & 2.7 & 1.4 & 1.4 & 1.0 & 2.4 & 1.1 & \\
\hline & orf19.3926 & 1.6 & 15.1 & 1.2 & 5.6 & 0.7 & 8.9 & 0.3 & 1.0 & endoribonuclease activity (IEA) \\
\hline & orf19.3976 & 1.5 & 2.0 & 0.8 & 0.9 & 1.6 & 1.0 & 1.5 & 1.0 & structural constituent of cytoskeleton (IEA) \\
\hline & orf19.4276 & 1.8 & 2.5 & 2.2 & 0.6 & 1.8 & 1.7 & 1.2 & 1.0 & \\
\hline & orf19.4711 & 3.8 & 1.7 & 4.9 & 1.4 & 1.7 & 1.0 & 3.7 & 0.9 & \\
\hline
\end{tabular}


Appendix B. Continued.

\begin{tabular}{|c|c|c|c|c|c|c|c|c|c|c|}
\hline \multirow[b]{3}{*}{ CGD Name } & \multirow[b]{3}{*}{ orf19 Designation } & \multicolumn{8}{|c|}{ Change in fold expression ${ }^{\mathrm{a}}$} & \multirow[b]{3}{*}{ Molecular function } \\
\hline & & \multicolumn{2}{|c|}{$\underline{\text { G648S }}$} & \multicolumn{2}{|c|}{$\underline{\text { G648D }}$} & \multicolumn{2}{|c|}{$\underline{\mathrm{Y} 642 \mathrm{~F}}$} & \multicolumn{2}{|c|}{$\underline{\mathrm{A} 643 \mathrm{~V}}$} & \\
\hline & & Exp1 & Exp2 & Exp1 & Exp2 & Exp1 & Exp2 & Exp1 & Exp2 & \\
\hline & orf19.4735 & 1.5 & 1.6 & 1.5 & 1.1 & 1.2 & 0.8 & 1.4 & 0.8 & \\
\hline & orf19.4923 & 1.5 & 2.4 & 1.4 & 1.9 & 1.1 & 1.1 & 1.3 & 1.9 & sugar transmembrane transporter activity (ISS) \\
\hline & orf19.5693 & 1.5 & 1.5 & 1.6 & 1.4 & 1.1 & 1.4 & 1.3 & 1.4 & GPI-anchor transamidase activity (IEA) \\
\hline & orf19.6351 & 1.7 & 1.7 & 1.3 & 1.4 & 2.4 & 0.9 & 1.5 & 1.0 & \\
\hline & orf19.6899 & 1.6 & 2.5 & 0.6 & 0.4 & 0.7 & 0.9 & 0.3 & 0.4 & \\
\hline & orf19.6950 & 1.5 & 2.2 & 0.9 & 1.0 & 1.5 & 1.2 & 1.1 & 1.0 & \\
\hline & orf19.6979 & 1.8 & 1.5 & 2.2 & 0.9 & 1.6 & 1.1 & 1.3 & 1.0 & \\
\hline & orf19.6982 & 1.7 & 1.5 & 1.4 & 1.3 & 1.7 & 1.1 & 1.4 & 1.1 & \\
\hline & $\begin{array}{l}\text { orf19.7193 } \\
\text { orf19.7303 }\end{array}$ & $\begin{array}{l}1.7 \\
3.5\end{array}$ & $\begin{array}{l}1.5 \\
2.8\end{array}$ & $\begin{array}{l}\mathbf{1 . 0} \\
1.8\end{array}$ & $\begin{array}{l}1.1 \\
0.7\end{array}$ & $\begin{array}{l}\mathbf{1 . 3} \\
1.4\end{array}$ & $\begin{array}{l}\mathbf{0 . 8} \\
1.2\end{array}$ & $\begin{array}{l}\mathbf{1 . 0} \\
3.8\end{array}$ & $\begin{array}{l}\mathbf{0 . 8} \\
1.3\end{array}$ & \\
\hline & orf19.911 & 2.3 & 1.6 & 1.8 & 1.2 & 1.4 & 1.2 & 1.9 & 0.9 & ubiquitin-protein ligase activity (IEA) \\
\hline & orf19.980 & 1.7 & 1.5 & 1.9 & 1.3 & 0.8 & 1.3 & 0.8 & 1.1 & \\
\hline$A D O 1$ & orf19.5591 & 1.4 & 1.3 & 1.6 & 1.6 & 1.4 & 1.5 & 1.5 & 1.4 & adenosine kinase activity (IEA) \\
\hline AMS1 & orf19.2768 & 1.1 & 2.8 & 4.2 & 2.0 & 1.3 & 0.8 & 1.3 & 1.3 & alpha-mannosidase activity (IEA) \\
\hline ASR1 & orf19.2344 & 0.6 & 3.2 & 2.1 & 1.8 & 0.6 & 1.0 & 0.9 & 1.2 & \\
\hline BUD16 & orf19.1828 & 2.5 & 0.9 & 2.7 & 1.5 & 2.1 & 0.8 & 2.1 & 0.9 & \\
\hline$C A S 1$ & orf19.1135 & 1.1 & 1.2 & 1.5 & 1.5 & 1.2 & 1.0 & 1.4 & 1.5 & \\
\hline$C A T 1$ & orf19.6229 & 1.2 & 1.7 & 1.7 & 1.8 & 1.3 & 1.5 & 1.4 & 1.8 & catalase activity (IDA, IMP, ISS)|protein binding (IDA) \\
\hline CAT2 & orf19.4591 & 1.1 & 1.4 & 1.5 & 1.6 & 1.1 & 1.1 & 1.5 & 1.4 & \\
\hline CAT8 & orf19.5097 & 1.6 & 0.7 & 2.0 & 2.1 & 1.0 & 1.1 & 2.4 & 1.1 & \\
\hline$C D C 23$ & orf19.6437 & 1.1 & 1.1 & 1.6 & 1.5 & 1.3 & 1.1 & 1.5 & 1.4 & protein binding (IEA)|ubiquitin-protein ligase activity (IEA) \\
\hline CTA4 & orf19.7374 & 1.4 & 1.3 & 2.0 & 1.6 & 1.7 & 1.4 & 1.9 & 1.3 & transcription activator activity (IDA, IGI) \\
\hline CYB2 & orf19.5000 & 1.1 & 1.3 & 1.8 & 1.5 & 1.2 & 0.9 & 1.5 & 1.1 & L-lactate dehydrogenase (cytochrome) activity (NAS) \\
\hline$D A L 9$ & orf19.6956 & 0.9 & 1.6 & 2.0 & 1.6 & 0.9 & 1.2 & 1.3 & 1.7 & anion:cation symporter activity (ISS) \\
\hline
\end{tabular}


Appendix B. Continued.

\begin{tabular}{|c|c|c|c|c|c|c|c|c|c|c|}
\hline \multirow[b]{3}{*}{ CGD Name } & \multirow[b]{3}{*}{ orf19 Designation } & \multicolumn{8}{|c|}{ Change in fold expression ${ }^{a}$} & \multirow[b]{3}{*}{ Molecular function } \\
\hline & & \multicolumn{2}{|c|}{$\underline{\text { G648S }}$} & \multicolumn{2}{|c|}{$\underline{\text { G648D }}$} & \multicolumn{2}{|c|}{$\underline{Y 642 F}$} & \multicolumn{2}{|c|}{$\underline{\mathrm{A} 643 \mathrm{~V}}$} & \\
\hline & & Exp1 & Exp2 & Exp1 & Exp2 & Exp1 & Exp2 & Exp1 & Exp2 & \\
\hline ECM29 & orf19.6773 & 1.1 & 1.2 & 1.5 & 1.5 & 1.3 & 1.0 & 1.2 & 1.2 & \\
\hline ERG10 & orf19.1591 & 1.2 & 1.5 & 2.0 & 1.7 & 1.1 & 1.0 & 1.7 & 1.4 & acetyl-CoA C-acyltransferase activity (IEA) \\
\hline ERG10 & orf19.1591 & 1.3 & 1.5 & 1.6 & 1.6 & 1.4 & 1.7 & 1.4 & 1.8 & acetyl-CoA C-acyltransferase activity (IEA) \\
\hline FGR16 & orf19.2303 & 2.4 & 1.1 & 1.8 & 1.5 & 1.7 & 1.0 & 2.1 & 1.2 & \\
\hline FGR34 & orf19.7579 & 1.0 & 1.4 & 1.5 & 1.5 & 1.0 & 0.9 & 1.2 & 1.2 & \\
\hline$F M O 2$ & orf19.859 & 1.3 & 1.8 & 2.5 & 1.9 & 0.6 & 0.7 & 1.5 & 1.2 & \\
\hline$F R P 2$ & orf19.7112 & 0.8 & 1.4 & 1.6 & 1.5 & 1.1 & 1.1 & 1.1 & 1.3 & ferric-chelate reductase activity (IEA) \\
\hline$G A P 2$ & orf19.6993 & 0.6 & 1.5 & 2.2 & 1.7 & 0.6 & 0.7 & 1.1 & 1.6 & L-proline transmembrane transporter activity (IEA) \\
\hline GAT1 & orf19.1275 & 0.7 & 1.8 & 1.6 & 2.0 & 0.5 & 1.0 & 0.9 & 2.7 & specific RNA polymerase II transcription factor activity (IMP, ISS) \\
\hline$G D H 3$ & orf19.4716 & 1.0 & 1.6 & 1.9 & 1.6 & 1.0 & 1.3 & 1.2 & 1.6 & glutamate dehydrogenase (NADP+) activity (IEA) \\
\hline GPD1 & orf19.1756 & 1.1 & 1.3 & 1.7 & 1.5 & 1.2 & 1.0 & 1.5 & 1.4 & glycerol-3-phosphate dehydrogenase (NAD+) activity (IEA) \\
\hline GRE2 & orf19.3150 & 0.9 & 1.5 & 1.7 & 1.5 & 1.0 & 1.2 & 1.2 & 1.4 & oxidoreductase activity (NAS) \\
\hline GST1 & orf19.3121 & 0.8 & 1.1 & 2.4 & 1.6 & 1.1 & 1.6 & 1.0 & 1.4 & transcription corepressor activity (IEA) \\
\hline HIS5 & orf19.4177 & 1.3 & 1.5 & 1.5 & 1.5 & 1.0 & 0.8 & 1.7 & 1.2 & histidinol-phosphate transaminase activity (IEA) \\
\hline HOS3 & orf19.2772 & 1.1 & 1.1 & 1.6 & 1.5 & 1.3 & 0.9 & 1.4 & 1.2 & histone deacetylase activity (ISS) \\
\hline$H S X 11$ & orf19.4592 & 2.0 & 1.4 & 2.4 & 1.6 & 1.9 & 1.1 & 2.1 & 1.2 & ceramide glucosyltransferase activity (IDA, IGI, IMP) \\
\hline$H X K 1$ & orf19.2154 & 1.3 & 1.4 & 1.6 & 1.6 & 0.9 & 1.0 & 1.3 & 1.5 & $\mathrm{~N}$-acetylglucosamine kinase activity (IDA, IMP)|hexokinase activity (ISS) \\
\hline IFRI & orf19.1763 & 1.1 & 3.3 & 4.0 & 1.9 & 1.6 & 1.1 & 2.1 & 1.4 & \\
\hline IFU5 & orf19.2568 & 1.1 & 1.7 & 2.2 & 1.7 & 1.1 & 1.2 & 1.3 & 1.6 & \\
\hline INO4 & orf19.837.1 & 1.1 & 1.0 & 1.6 & 1.7 & 1.4 & 1.1 & 1.7 & 1.2 & \\
\hline INP51 & orf19.1373 & 1.9 & 1.0 & 2.5 & 1.5 & 1.7 & 0.8 & 2.6 & 1.3 & inositol-polyphosphate 5-phosphatase activity (IEA) \\
\hline KNS1 & orf19.4979 & 0.7 & 1.8 & 2.0 & 2.2 & 0.9 & 0.8 & 1.4 & 2.1 & protein serine/threonine kinase activity (IEA) \\
\hline LEU2 & orf19.7080 & 1.0 & 1.5 & 1.5 & 1.5 & 0.9 & 1.2 & 1.1 & 1.3 & 3-isopropylmalate dehydrogenase activity (IDA, IGI, NAS) \\
\hline LIP8 & orf19.1345 & 1.4 & 1.9 & 1.6 & 1.6 & 0.8 & 1.5 & 1.3 & 2.0 & lipase activity (IDA) \\
\hline
\end{tabular}


Appendix B. Continued.

\begin{tabular}{|c|c|c|c|c|c|c|c|c|c|c|}
\hline \multirow[b]{3}{*}{ CGD Name } & \multirow[b]{3}{*}{ orf19 Designation } & \multicolumn{8}{|c|}{ Change in fold expression ${ }^{\mathrm{a}}$} & \multirow[b]{3}{*}{ Molecular function } \\
\hline & & \multicolumn{2}{|c|}{$\underline{\text { G648S }}$} & \multicolumn{2}{|c|}{$\underline{\text { G648D }}$} & \multicolumn{2}{|c|}{$\underline{\mathrm{Y} 642 \mathrm{~F}}$} & \multicolumn{2}{|c|}{$\underline{\mathrm{A} 643 \mathrm{~V}}$} & \\
\hline & & Exp1 & Exp2 & Exp1 & Exp2 & Exp1 & Exp2 & Exp1 & Exp2 & \\
\hline MIT1 & orf19.4077 & 1.2 & 1.1 & 2.2 & 1.9 & 1.4 & 1.0 & 1.8 & 1.3 & mannosyltransferase activity (IMP) \\
\hline$M L S 1$ & orf19.4833 & 0.8 & 2.1 & 1.9 & 1.5 & 0.8 & 1.0 & 1.0 & 1.3 & malate synthase activity (ISS) \\
\hline$M U Q 1$ & orf19.2107 & 0.9 & 1.5 & 1.6 & 1.5 & 1.1 & 1.1 & 1.3 & 1.4 & choline-phosphate cytidylyltransferase activity (IEA) \\
\hline NAG6 & orf19.2151 & 1.2 & 1.3 & 1.7 & 1.5 & 1.1 & 1.1 & 1.4 & 1.3 & GTP binding (ISS) \\
\hline NCE4 & orf19.5320 & 1.2 & 0.8 & 1.8 & 1.6 & 1.6 & 0.8 & 2.0 & 1.1 & \\
\hline NUF2 & orf19.1941 & 1.1 & 1.1 & 1.6 & 1.5 & 1.4 & 0.8 & 1.6 & 1.1 & structural constituent of cytoskeleton (IEA) \\
\hline OYE32 & orf19.3131 & 1.0 & 2.5 & 2.3 & 1.7 & 1.0 & 1.2 & 1.3 & 1.8 & NADPH dehydrogenase activity (IEA) \\
\hline PAN1 & orf19.886 & 1.1 & 1.8 & 1.8 & 1.9 & 1.0 & 1.3 & 1.3 & 1.7 & cytoskeletal adaptor activity (IEA)|protein binding, bridging (IEA) \\
\hline PDC12 & orf19.4608 & 2.3 & 1.3 & 3.8 & 1.5 & 3.0 & 1.0 & 3.3 & 1.0 & \\
\hline$P D K 2$ & orf19.7281 & 0.9 & 1.3 & 1.5 & 1.6 & 1.0 & 0.8 & 1.2 & 1.3 & \\
\hline PEP7 & orf19.5662 & 1.5 & 1.1 & 1.9 & 1.7 & 1.7 & 0.8 & 1.6 & 1.4 & phosphatidylinositol 3-phosphate binding (IEA) \\
\hline PGA60 & orf19.5588 & 0.8 & 4.6 & 1.7 & 2.4 & 1.0 & 0.2 & 1.0 & 5.0 & \\
\hline PLB5 & orf19.5102 & 0.9 & 1.6 & 1.9 & 1.5 & 0.8 & 0.9 & 1.2 & 1.5 & phospholipase A2 activity (IMP)|lysophospholipase activity (ISS) \\
\hline$P L D 1$ & orf19.1161 & 1.3 & 1.2 & 1.8 & 1.5 & 1.3 & 1.1 & 1.7 & 1.4 & phospholipase D activity (IMP, ISS) \\
\hline POT1-2 & orf19.2046 & 1.1 & 1.5 & 1.5 & 1.7 & 1.2 & 1.0 & 1.5 & 1.3 & acetyl-CoA C-acyltransferase activity (IEA) \\
\hline PPH3 & orf19.4378 & 1.2 & 1.2 & 1.5 & 1.5 & 1.2 & 1.0 & 1.8 & 1.4 & \\
\hline PRR2 & orf19.1341 & 1.7 & 1.4 & 3.3 & 1.5 & 0.6 & 1.9 & 2.7 & 0.8 & receptor signaling protein serine/threonine kinase activity (IEA) \\
\hline RAD10 & orf19.2469 & 1.7 & 1.2 & 1.5 & 1.5 & 1.7 & 1.1 & 1.6 & 1.1 & DNA strand annealing activity (ISA) \\
\hline RAD14 & orf19.6517 & 1.0 & 1.5 & 1.6 & 1.6 & 0.9 & 1.0 & 1.2 & 1.7 & damaged DNA binding (IEA)|zinc ion binding (IEA) \\
\hline$R A M 1$ & orf19.5046 & 1.0 & 0.9 & 1.5 & 1.5 & 1.0 & 1.0 & 0.9 & 1.0 & protein farnesyltransferase activity (IEA) \\
\hline RDH54 & orf19.5367 & 1.5 & 1.2 & 1.7 & 1.6 & 1.2 & 0.9 & 1.6 & 1.1 & DNA topoisomerase activity (IEA)|DNA translocase activity (IEA) \\
\hline$R G A 2$ & orf19.4593 & 1.2 & 1.2 & 1.6 & 1.6 & 1.4 & 1.1 & 1.4 & 1.4 & Rho GTPase activator activity (IDA)|signal transducer activity (IEA) \\
\hline$R G S 2$ & orf19.695 & 0.9 & 0.8 & 1.5 & 2.6 & 0.7 & 0.5 & 1.0 & 1.6 & \\
\hline RIBI & orf19.2862 & 1.3 & 1.3 & 1.8 & 1.5 & 1.3 & 1.1 & 1.7 & 1.4 & cyclohydrolase activity (IEA) \\
\hline
\end{tabular}


Appendix B. Continued.

\begin{tabular}{|c|c|c|c|c|c|c|c|c|c|c|}
\hline \multirow[b]{3}{*}{ CGD Name } & \multirow[b]{3}{*}{ orf19 Designation } & \multicolumn{8}{|c|}{ Change in fold expression ${ }^{a}$} & \multirow[b]{3}{*}{ Molecular function } \\
\hline & & \multicolumn{2}{|c|}{$\underline{\text { G648S }}$} & \multicolumn{2}{|c|}{$\underline{\text { G648D }}$} & \multicolumn{2}{|c|}{$\underline{\mathrm{Y} 642 \mathrm{~F}}$} & \multicolumn{2}{|c|}{$\underline{\mathrm{A} 643 \mathrm{~V}}$} & \\
\hline & & Exp1 & Exp2 & Exp1 & Exp2 & Exp1 & Exp2 & Exp1 & Exp2 & \\
\hline RPN10 & orf19.4102 & 1.3 & 1.2 & 1.5 & 1.6 & 1.4 & 1.2 & 1.5 & 1.3 & endopeptidase activity (IEA)|protein binding, bridging (IEA) \\
\hline RVS162 & orf19.6349 & 1.1 & 1.7 & 1.8 & 1.5 & 1.2 & 0.9 & 1.7 & 1.3 & cytoskeletal protein binding (IEA) \\
\hline SKN7 & orf19.971 & 1.2 & 1.5 & 1.7 & 1.7 & 1.1 & 1.0 & 1.3 & 1.5 & two-component response regulator activity (ISS) \\
\hline$S M P 2$ & orf19.1462 & 1.1 & 1.0 & 1.7 & 1.5 & 1.4 & 0.9 & 1.6 & 1.1 & phosphatidate phosphatase activity (IEA) \\
\hline SNF3 & orf19.5962 & 1.5 & 1.1 & 1.9 & 1.5 & 1.5 & 1.1 & 1.9 & 1.4 & glucose transmembrane transporter activity (ISS) \\
\hline$S N Z 1$ & orf19.2947 & 1.0 & 1.6 & 1.7 & 1.9 & 1.3 & 1.4 & 1.3 & 1.6 & protein binding (IEA) \\
\hline SSP96 & orf19.5145 & 0.9 & 1.9 & 1.5 & 2.3 & 1.3 & 1.2 & 1.3 & 2.1 & \\
\hline SVF1 & orf19.6068 & 1.3 & 1.1 & 1.9 & 1.6 & 1.5 & 1.1 & 1.5 & 1.4 & \\
\hline$T U P 1$ & orf19.6109 & 1.2 & 1.7 & 1.7 & 1.6 & 1.0 & 1.3 & 1.3 & 1.4 & transcription repressor activity (IGI, IMP, ISS) \\
\hline$U B C 15$ & orf19.5337 & 1.4 & 1.4 & 1.8 & 1.5 & 1.3 & 1.0 & 1.7 & 1.4 & \\
\hline$U G A 1$ & orf19.802 & 1.2 & 1.8 & 1.8 & 1.5 & 1.1 & 1.1 & 1.4 & 1.3 & 4-aminobutyrate transaminase activity (IEA) \\
\hline VPS2 & orf19.945 & 1.3 & 1.7 & 2.1 & 1.9 & 1.6 & 1.3 & 1.8 & 1.4 & \\
\hline$X Y L 2$ & orf19.7676 & 0.8 & 2.0 & 1.9 & 1.6 & 0.8 & 1.1 & 1.2 & 1.6 & L-iditol 2-dehydrogenase activity (IEA) \\
\hline \multirow[t]{11}{*}{ YAF9 } & orf19.5501 & 0.9 & 1.5 & 1.5 & 1.5 & 0.7 & 1.1 & 1.7 & 1.3 & contributes_to histone acetyltransferase activity (IC) \\
\hline & orf19.1070 & 1.0 & 1.2 & 3.9 & 2.2 & 1.2 & 0.9 & 1.7 & 1.2 & \\
\hline & orf19.1191 & 1.1 & 1.3 & 1.6 & 1.5 & 1.1 & 1.0 & 1.5 & 1.4 & contributes_to ubiquitin-protein ligase activity (IEA) \\
\hline & orf19.1314 & 1.3 & 0.9 & 1.6 & 1.7 & 1.4 & 1.0 & 2.0 & 1.4 & \\
\hline & orf19.1364 & 1.2 & 1.4 & 2.0 & 1.6 & 1.1 & 0.9 & 1.5 & 1.2 & \\
\hline & orf19.1369 & 1.1 & 1.7 & 1.9 & 1.6 & 0.9 & 1.2 & 0.9 & 1.4 & \\
\hline & orf19.1392 & 1.5 & 1.2 & 1.5 & 1.7 & 1.9 & 1.0 & 2.0 & 1.3 & chaperone binding (IEA)|protein disulfide isomerase activity (IEA) \\
\hline & orf19.1395 & 1.2 & 1.9 & 1.5 & 1.8 & 1.3 & 0.7 & 0.9 & 1.8 & inorganic phosphate transmembrane transporter activity (IEA) \\
\hline & orf19.1406 & 1.0 & 1.0 & 2.1 & 1.5 & 1.1 & 0.7 & 1.8 & 0.9 & \\
\hline & orf19.1421 & 1.0 & 1.8 & 1.7 & 1.5 & 1.1 & 1.0 & 1.2 & 1.3 & ureidoglycolate hydrolase activity (IEA) \\
\hline & orf19.1434 & 1.0 & 1.0 & 1.9 & 1.7 & 1.0 & 1.0 & 1.3 & 1.0 & kinase activator activity (IEA)|protein binding (IEA) \\
\hline
\end{tabular}


Appendix B. Continued.

\begin{tabular}{|c|c|c|c|c|c|c|c|c|c|c|}
\hline \multirow[b]{3}{*}{ CGD Name } & \multirow[b]{3}{*}{ orf19 Designation } & \multicolumn{8}{|c|}{ Change in fold expression ${ }^{\mathrm{a}}$} & \multirow[b]{3}{*}{ Molecular function } \\
\hline & & \multicolumn{2}{|c|}{$\underline{\text { G648S }}$} & \multicolumn{2}{|c|}{$\underline{\text { G648D }}$} & \multicolumn{2}{|c|}{$\underline{\mathrm{Y} 642 \mathrm{~F}}$} & \multicolumn{2}{|c|}{$\underline{\mathrm{A} 643 \mathrm{~V}}$} & \\
\hline & & Exp1 & Exp2 & Exp1 & Exp2 & Exp1 & Exp2 & Exp1 & Exp2 & \\
\hline & orf19.1482 & 1.0 & 1.4 & 1.6 & 1.8 & 0.9 & 1.0 & 1.3 & 1.4 & \\
\hline & orf19.1496 & 1.3 & 1.1 & 1.6 & 1.6 & 1.2 & 0.8 & 1.3 & 1.4 & transcription factor activity (ISS) \\
\hline & orf19.1667 & 1.3 & 1.8 & 1.5 & 1.8 & 1.3 & 1.7 & 1.1 & 1.7 & helicase activity (IEA) \\
\hline & orf19.1723 & 0.9 & 1.4 & 1.5 & 1.5 & 1.0 & 1.1 & 1.3 & 1.4 & \\
\hline & orf19.1728 & 1.1 & 5.8 & 2.1 & 3.3 & 1.0 & 0.2 & 0.4 & 1.6 & \\
\hline & orf19.1730 & 1.1 & 1.0 & 1.6 & 1.7 & 1.5 & 1.0 & 1.4 & 1.3 & \\
\hline & orf19.1776 & 2.6 & 1.1 & 3.1 & 2.1 & 4.0 & 1.1 & 4.3 & 1.3 & \\
\hline & orf19.190 & 1.2 & 1.1 & 1.5 & 1.6 & 1.3 & 0.7 & 1.1 & 1.0 & \\
\hline & orf19.1917 & 1.3 & 1.5 & 1.9 & 1.5 & 1.2 & 1.2 & 1.4 & 1.5 & \\
\hline & orf19.192 & 1.3 & 1.4 & 1.6 & 1.8 & 1.2 & 0.9 & 1.4 & 1.4 & \\
\hline & orf19.2009 & 2.1 & 1.0 & 2.6 & 1.6 & 2.8 & 0.8 & 2.6 & 1.3 & protein binding (IEA) \\
\hline & orf19.2065 & 1.1 & 1.6 & 1.9 & 1.5 & 1.3 & 1.1 & 1.4 & 1.6 & allantoicase activity (IEA) \\
\hline & orf19.2204.2 & 1.5 & 1.2 & 2.4 & 1.5 & 1.3 & 1.3 & 1.9 & 1.4 & GTPase activity (ISS)|signal transducer activity (IDA) \\
\hline & orf19.225 & 1.1 & 1.4 & 2.0 & 1.6 & 1.3 & 1.0 & 1.2 & 1.2 & \\
\hline & orf19.2257 & 1.2 & 1.3 & 1.5 & 1.6 & 1.3 & 1.2 & 1.4 & 1.3 & \\
\hline & orf19.2262 & 0.9 & 1.9 & 1.9 & 1.7 & 0.9 & 1.0 & 1.3 & 1.6 & AU-rich element binding (IEA) \\
\hline & orf19.2313 & 1.3 & 1.6 & 1.7 & 1.7 & 0.9 & 1.0 & 1.4 & 1.7 & \\
\hline & orf19.2346 & 1.6 & 1.0 & 2.0 & 1.5 & 1.7 & 0.8 & 1.7 & 1.2 & \\
\hline & orf19.2411 & 1.1 & 1.3 & 1.5 & 1.7 & 1.3 & 1.1 & 1.5 & 1.4 & SNAP receptor activity (IEA) \\
\hline & orf19.2496 & 1.0 & 1.3 & 1.7 & 1.7 & 0.8 & 1.6 & 1.4 & 1.4 & \\
\hline & orf19.2565 & 1.1 & 1.3 & 1.5 & 1.6 & 0.8 & 0.9 & 1.3 & 1.5 & phosphatidylinositol 3-phosphate binding (IEA) \\
\hline & orf19.268 & 0.9 & 1.4 & 1.8 & 1.5 & 1.0 & 0.7 & 1.3 & 1.5 & \\
\hline & orf19.2710 & 1.5 & 1.0 & 2.8 & 1.5 & 2.1 & 0.8 & 2.4 & 1.1 & enzyme activator activity (IEA) \\
\hline & orf19.273 & 1.2 & 1.2 & 1.8 & 1.5 & 1.2 & 1.3 & 1.4 & 1.4 & \\
\hline
\end{tabular}


Appendix B. Continued.

\begin{tabular}{|c|c|c|c|c|c|c|c|c|c|c|}
\hline \multirow[b]{3}{*}{ CGD Name } & \multirow[b]{3}{*}{ orf19 Designation } & \multicolumn{8}{|c|}{ Change in fold expression ${ }^{a}$} & \multirow[b]{3}{*}{ Molecular function } \\
\hline & & \multicolumn{2}{|c|}{$\underline{\text { G648S }}$} & \multicolumn{2}{|c|}{$\underline{\text { G648D }}$} & \multicolumn{2}{|c|}{$\underline{\mathrm{Y} 642 \mathrm{~F}}$} & \multicolumn{2}{|c|}{$\underline{\mathrm{A} 643 \mathrm{~V}}$} & \\
\hline & & Exp1 & Exp2 & Exp1 & Exp2 & Exp1 & Exp2 & Exp1 & Exp2 & \\
\hline & orf19.2740 & 1.3 & 1.6 & 1.5 & 1.5 & 1.1 & 1.1 & 1.1 & 1.4 & \\
\hline & orf19.2755 & 1.6 & 0.9 & 2.0 & 1.6 & 2.0 & 0.9 & 1.6 & 0.7 & endopeptidase activity (IEA) \\
\hline & orf19.2791 & 1.1 & 1.6 & 1.7 & 1.5 & 1.0 & 1.0 & 1.3 & 1.4 & myosin I binding (IEA) \\
\hline & orf19.2888 & 1.4 & 0.7 & 1.8 & 1.6 & 2.2 & 0.8 & 2.6 & 1.0 & \\
\hline & orf19.2905 & 1.1 & 1.6 & 1.8 & 2.1 & 1.5 & 1.2 & 1.3 & 1.6 & \\
\hline & orf19.2913 & 1.1 & 1.1 & 1.6 & 1.5 & 1.1 & 1.0 & 1.4 & 1.3 & \\
\hline & orf19.2974 & 1.2 & 1.1 & 1.5 & 1.5 & 1.6 & 1.1 & 1.7 & 1.3 & palmitoyltransferase activity (IEA) \\
\hline & orf19.3151 & 0.8 & 1.4 & 1.5 & 1.5 & 1.1 & 0.9 & 1.6 & 1.1 & oxidoreductase activity (NAS) \\
\hline & orf19.3214 & 1.2 & 1.5 & 2.5 & 2.3 & 1.3 & 1.1 & 1.7 & 1.1 & \\
\hline & orf19.3215 & 1.3 & 1.6 & 1.8 & 1.5 & 1.2 & 1.0 & 1.5 & 1.2 & \\
\hline & orf19.3407 & 1.3 & 1.4 & 1.5 & 1.6 & 1.4 & 1.0 & 1.5 & 1.2 & transcription factor activity (ISS) \\
\hline & orf19.3411 & 1.4 & 1.1 & 1.7 & 1.5 & 1.3 & 1.0 & 1.8 & 1.4 & \\
\hline & orf19.345 & 1.6 & 1.3 & 1.5 & 1.7 & 1.4 & 1.6 & 1.4 & 1.3 & succinate-semialdehyde dehydrogenase $[\mathrm{NAD}(\mathrm{P})+]$ activity $(\mathrm{IEA})$ \\
\hline & orf19.3536 & 1.4 & 1.2 & 1.7 & 1.7 & 1.4 & 1.2 & 1.6 & 1.4 & acetylglucosaminyltransferase activity (IEA) \\
\hline & orf19.3631 & 1.6 & 1.4 & 1.5 & 1.5 & 1.7 & 1.2 & 1.0 & 1.3 & \\
\hline & orf19.3658 & 1.0 & 1.5 & 1.5 & 1.8 & 1.0 & 1.3 & 1.4 & 1.5 & \\
\hline & orf19.3661 & 1.3 & 1.4 & 1.7 & 1.7 & 1.6 & 1.1 & 1.4 & 1.4 & ubiquitin-specific protease activity (IEA) \\
\hline & orf19.3679 & 1.0 & 1.7 & 2.2 & 1.5 & 1.0 & 0.9 & 1.5 & 1.4 & \\
\hline & orf19.3804 & 1.3 & 1.2 & 1.9 & 1.7 & 1.4 & 1.2 & 1.5 & 1.4 & \\
\hline & orf19.3806 & 1.4 & 1.2 & 2.0 & 1.5 & 2.0 & 0.9 & 2.0 & 1.4 & \\
\hline & orf19.3814 & 1.1 & 1.4 & 1.8 & 1.7 & 1.1 & 0.8 & 1.6 & 1.4 & \\
\hline & orf19.393 & 1.3 & 1.2 & 1.8 & 1.5 & 1.3 & 1.0 & 1.8 & 1.4 & \\
\hline & orf19.3939 & 2.2 & 1.1 & 2.1 & 1.5 & 2.5 & 1.1 & 2.4 & 1.2 & \\
\hline & orf19.3984 & 1.0 & 1.0 & 1.6 & 1.8 & 1.3 & 0.8 & 1.3 & 1.2 & \\
\hline
\end{tabular}


Appendix B. Continued.

\begin{tabular}{|c|c|c|c|c|c|c|c|c|c|c|}
\hline \multirow[b]{3}{*}{ CGD Name } & \multirow[b]{3}{*}{ orf19 Designation } & \multicolumn{8}{|c|}{ Change in fold expression ${ }^{\mathrm{a}}$} & \multirow[b]{3}{*}{ Molecular function } \\
\hline & & \multicolumn{2}{|c|}{$\underline{\text { G648S }}$} & \multicolumn{2}{|c|}{$\underline{\text { G648D }}$} & \multicolumn{2}{|c|}{$\underline{\mathrm{Y} 642 \mathrm{~F}}$} & \multicolumn{2}{|c|}{$\underline{\mathrm{A} 643 \mathrm{~V}}$} & \\
\hline & & Exp1 & Exp2 & Exp1 & Exp2 & Exp1 & Exp2 & Exp1 & Exp2 & \\
\hline & orf19.4061 & 1.5 & 1.2 & 1.8 & 1.5 & 1.2 & 1.0 & 2.1 & 1.3 & \\
\hline & orf19.4206 & 1.3 & 1.2 & 2.2 & 2.2 & 1.6 & 1.0 & 1.9 & 1.4 & crossover junction endodeoxyribonuclease activity (IEA) \\
\hline & orf19.4229 & 0.4 & 1.8 & 2.0 & 1.6 & 0.8 & 1.1 & 0.9 & 1.4 & bis(5'-adenosyl)-hexaphosphatase activity (IEA) \\
\hline & orf19.4246 & 1.4 & 1.3 & 2.2 & 1.5 & 1.6 & 1.2 & 1.9 & 1.4 & \\
\hline & orf19.4357 & 1.6 & 1.2 & 2.1 & 1.5 & 1.5 & 1.0 & 1.7 & 1.2 & misfolded protein binding (IEA) \\
\hline & orf19.4390 & 0.8 & 1.4 & 1.7 & 1.6 & 0.9 & 0.9 & 1.2 & 1.4 & \\
\hline & orf19.4503 & 1.4 & 1.1 & 1.8 & 1.5 & 1.5 & 1.3 & 2.0 & 1.3 & \\
\hline & orf19.4583 & 2.3 & 1.1 & 1.9 & 1.6 & 1.8 & 1.2 & 2.0 & 0.8 & transporter activity (IEA) \\
\hline & orf19.4691 & 1.2 & 1.4 & 2.5 & 2.0 & 0.9 & 0.8 & 1.0 & 1.3 & \\
\hline & orf19.4726 & 1.3 & 1.2 & 2.0 & 1.5 & 1.6 & 1.2 & 1.8 & 1.4 & calcium ion binding (IEA)|enzyme activator activity (IEA) \\
\hline & orf19.4731 & 1.4 & 1.0 & 1.8 & 1.6 & 1.7 & 1.1 & 1.9 & 1.4 & \\
\hline & orf19.4791 & 1.2 & 1.8 & 2.3 & 1.6 & 1.0 & 0.7 & 1.5 & 1.1 & \\
\hline & orf19.4807 & 1.2 & 1.1 & 1.5 & 1.5 & 1.0 & 0.9 & 1.3 & 1.2 & inorganic diphosphatase activity (IEA) \\
\hline & orf19.4894 & 1.0 & 1.9 & 1.6 & 1.5 & 1.0 & 1.0 & 1.2 & 1.3 & 0.0 \\
\hline & orf19.4983 & 1.6 & 1.2 & 1.6 & 1.6 & 2.0 & 1.0 & 1.7 & 1.2 & 0.0 \\
\hline & orf19.5008.1 & 0.9 & 1.1 & 1.5 & 1.9 & 1.1 & 0.8 & 1.3 & 1.0 & structural constituent of cytoskeleton (IEA) \\
\hline & orf19.5014 & 1.1 & 1.1 & 1.5 & 1.6 & 1.0 & 0.9 & 1.2 & 1.2 & 0.0 \\
\hline & orf19.5095 & 1.1 & 1.4 & 2.2 & 1.9 & 1.1 & 1.0 & 1.4 & 1.4 & oxysterol binding (IEA)|sterol carrier activity (IEA) \\
\hline & orf19.5169 & 0.8 & 1.9 & 2.4 & 2.0 & 1.5 & 1.4 & 0.6 & 1.6 & amidase activity (IEA) \\
\hline & orf19.52 & 1.0 & 1.3 & 1.5 & 1.6 & 1.1 & 1.1 & 1.4 & 1.3 & 0.0 \\
\hline & orf19.5236.1 & 1.2 & 1.0 & 1.5 & 1.5 & 1.5 & 1.0 & 1.7 & 1.3 & dehydrodolichyl diphosphate synthase activity (IEA) \\
\hline & orf19.5253 & 1.1 & 1.1 & 1.6 & 1.5 & 1.5 & 1.0 & 1.9 & 1.4 & protein kinase activity (IEA) \\
\hline & orf19.5270 & 1.1 & 1.4 & 2.0 & 1.5 & 0.8 & 0.4 & 1.9 & 1.3 & \\
\hline & orf19.5342 & 1.0 & 1.5 & 1.6 & 1.5 & 1.0 & 1.1 & 1.3 & 1.3 & \\
\hline
\end{tabular}


Appendix B. Continued.

\begin{tabular}{|c|c|c|c|c|c|c|c|c|c|c|}
\hline \multirow[b]{3}{*}{ CGD Name } & \multirow[b]{3}{*}{ orf19 Designation } & \multicolumn{8}{|c|}{ Change in fold expression ${ }^{\mathrm{a}}$} & \multirow[b]{3}{*}{ Molecular function } \\
\hline & & \multicolumn{2}{|c|}{$\underline{\text { G648S }}$} & \multicolumn{2}{|c|}{$\underline{\text { G648D }}$} & \multicolumn{2}{|c|}{$\underline{\mathrm{Y} 642 \mathrm{~F}}$} & \multicolumn{2}{|c|}{$\underline{\mathrm{A} 643 \mathrm{~V}}$} & \\
\hline & & Exp1 & Exp2 & Exp1 & Exp2 & Exp1 & Exp2 & Exp1 & Exp2 & \\
\hline & orf19.5345 & 1.3 & 1.4 & 1.6 & 1.6 & 1.2 & 1.2 & 1.6 & 1.3 & protein binding, bridging (IEA) \\
\hline & orf19.537 & 1.8 & 1.3 & 1.9 & 1.6 & 1.7 & 1.4 & 2.5 & 1.4 & \\
\hline & orf19.5547 & 3.1 & 0.9 & 1.7 & 1.6 & 5.7 & 1.3 & 3.4 & 0.9 & \\
\hline & orf19.5620 & 1.2 & 1.7 & 1.6 & 1.5 & 1.2 & 1.4 & 1.3 & 1.6 & \\
\hline & orf19.5773 & 1.4 & 1.5 & 2.0 & 1.5 & 1.4 & 1.3 & 1.6 & 1.4 & dipeptidyl-peptidase activity (IEA) \\
\hline & orf19.5784 & 1.0 & 1.8 & 1.7 & 1.5 & 0.9 & 0.7 & 1.1 & 1.7 & \\
\hline & orf19.5812 & 1.2 & 1.1 & 1.6 & 1.5 & 1.3 & 1.2 & 1.5 & 1.4 & \\
\hline & orf19.5862 & 0.5 & 2.8 & 2.4 & 1.7 & 0.9 & 0.9 & 1.7 & 0.7 & arginase activity (IEA) \\
\hline & orf19.5881 & 1.4 & 1.2 & 2.1 & 1.6 & 1.4 & 0.9 & 1.7 & 1.1 & voltage-gated chloride channel activity (IEA) \\
\hline & orf19.589 & 1.2 & 1.3 & 2.0 & 1.6 & 1.3 & 1.2 & 1.6 & 1.4 & GTPase activity (ISS)|GTP binding (IEA) \\
\hline & orf19.5935 & 1.3 & 2.5 & 1.8 & 1.7 & 1.2 & 1.1 & 1.4 & 1.2 & \\
\hline & orf19.5952 & 1.1 & 1.0 & 1.6 & 1.6 & 1.1 & 0.9 & 1.1 & 1.6 & \\
\hline & orf19.5961 & 0.9 & 1.2 & 1.5 & 1.5 & 1.3 & 0.9 & 1.5 & 1.3 & \\
\hline & orf19.6045 & 1.2 & 1.2 & 1.6 & 1.5 & 1.1 & 1.1 & 1.4 & 1.4 & phosphatidylserine decarboxylase activity (IEA) \\
\hline & orf19.6187 & 1.3 & 1.6 & 1.7 & 1.6 & 1.4 & 1.2 & 1.4 & 1.3 & \\
\hline & orf19.6196 & 0.8 & 1.6 & 1.7 & 1.6 & 1.0 & 0.9 & 1.2 & 1.7 & \\
\hline & orf19.6200 & 1.3 & 1.3 & 2.7 & 1.7 & 1.6 & 0.8 & 2.1 & 1.4 & \\
\hline & orf19.6268 & 1.7 & 1.1 & 1.8 & 1.5 & 2.0 & 1.1 & 1.8 & 1.2 & \\
\hline & orf19.6357 & 2.2 & 1.2 & 2.0 & 1.6 & 2.1 & 1.2 & 2.6 & 1.4 & \\
\hline & orf19.6460 & 1.5 & 1.0 & 1.7 & 1.5 & 1.6 & 0.8 & 1.7 & 1.1 & ATPase activity (IEA)|protein heterodimerization activity (IEA) \\
\hline & orf19.6462 & 0.9 & 1.0 & 1.5 & 2.1 & 1.2 & 1.1 & 1.2 & 1.4 & \\
\hline & orf19.6498 & 1.3 & 1.1 & 1.8 & 1.5 & 1.4 & 1.0 & 1.8 & 1.4 & \\
\hline & orf19.6552 & 1.4 & 1.2 & 1.5 & 1.5 & 1.5 & 1.1 & 1.6 & 1.2 & thiol oxidase activity (IEA) \\
\hline & orf19.6556 & 1.1 & 1.1 & 1.7 & 1.5 & 1.1 & 0.9 & 1.0 & 0.9 & \\
\hline
\end{tabular}


Appendix B. Continued.

\begin{tabular}{|c|c|c|c|c|c|c|c|c|c|c|}
\hline \multirow[b]{3}{*}{ CGD Name } & \multirow[b]{3}{*}{ orf19 Designation } & \multicolumn{8}{|c|}{ Change in fold expression ${ }^{\mathrm{a}}$} & \multirow[b]{3}{*}{ Molecular function } \\
\hline & & \multicolumn{2}{|c|}{$\underline{\text { G648S }}$} & \multicolumn{2}{|c|}{$\underline{\text { G648D }}$} & \multicolumn{2}{|c|}{$\underline{Y 642 F}$} & \multicolumn{2}{|c|}{$\underline{\mathrm{A} 643 \mathrm{~V}}$} & \\
\hline & & Exp1 & Exp2 & Exp1 & Exp2 & Exp1 & Exp2 & Exp1 & Exp2 & \\
\hline & orf19.6569 & 1.9 & 1.1 & 2.1 & 1.7 & 1.5 & 1.1 & 2.6 & 1.3 & \\
\hline & orf19.6601 & 0.9 & 1.6 & 2.8 & 1.6 & 1.1 & 0.7 & 1.4 & 1.2 & \\
\hline & orf19.6709 & 1.0 & 1.4 & 1.6 & 1.6 & 0.8 & 1.1 & 1.3 & 1.3 & \\
\hline & orf19.6754 & 0.9 & 1.2 & 1.5 & 1.5 & 1.1 & 1.1 & 1.3 & 1.3 & \\
\hline & orf19.6867 & 1.2 & 1.5 & 1.7 & 1.7 & 1.5 & 1.2 & 1.5 & 1.4 & \\
\hline & orf19.6883 & 1.1 & 1.3 & 1.7 & 1.6 & 1.3 & 1.3 & 1.4 & 1.5 & cholesterol binding (IEA \\
\hline & orf19.6900 & 1.2 & 1.5 & 1.7 & 1.7 & 1.4 & 1.1 & 1.4 & 1.3 & \\
\hline & orf19.6905 & 1.1 & 1.1 & 1.5 & 1.5 & 1.3 & 1.0 & 1.4 & 1.3 & \\
\hline & orf19.7061 & 2.0 & 1.0 & 2.0 & 1.5 & 2.1 & 1.4 & 2.3 & 1.3 & ATPase activity (IEA) \\
\hline & orf19.711 & 0.9 & 2.0 & 1.6 & 2.0 & 0.8 & 1.4 & 1.1 & 1.2 & \\
\hline & orf19.7179 & 1.3 & 1.1 & 1.5 & 1.5 & 1.4 & 1.0 & 1.7 & 1.4 & \\
\hline & orf19.7225 & 1.2 & 1.1 & 2.1 & 1.5 & 1.0 & 0.9 & 1.8 & 1.3 & \\
\hline & orf19.7229 & 1.1 & 1.5 & 1.8 & 1.8 & 1.2 & 1.1 & 1.4 & 1.5 & \\
\hline & orf19.7260 & 0.8 & 2.0 & 1.5 & 2.2 & 0.7 & 1.4 & 0.9 & 1.7 & \\
\hline & orf19.7265 & 1.1 & 1.6 & 1.6 & 1.7 & 1.2 & 1.3 & 1.2 & 1.5 & \\
\hline & orf19.7267 & 1.5 & 1.4 & 1.5 & 1.6 & 1.4 & 1.2 & 1.4 & 1.3 & \\
\hline & orf19.7288 & 1.1 & 1.4 & 1.6 & 1.5 & 1.1 & 0.9 & 1.4 & 1.4 & \\
\hline & orf19.7328 & 1.3 & 1.5 & 1.9 & 1.6 & 1.5 & 1.3 & 1.3 & 1.5 & \\
\hline & orf19.7365 & 1.2 & 1.0 & 1.7 & 1.5 & 1.1 & 0.9 & 1.1 & 0.9 & \\
\hline & orf19.7449 & 1.3 & 1.2 & 1.8 & 1.7 & 1.5 & 1.3 & 1.9 & 1.3 & \\
\hline & orf19.7507 & 0.9 & 1.3 & 1.6 & 1.7 & 1.3 & 0.8 & 1.1 & 1.3 & \\
\hline & orf19.751 & 1.2 & 2.6 & 1.6 & 1.9 & 1.0 & 1.7 & 1.3 & 2.6 & \\
\hline & orf19.7538 & 1.2 & 1.5 & 1.5 & 1.7 & 1.2 & 1.2 & 1.4 & 1.5 & DNA helicase activity (IEA) \\
\hline & orf19.834 & 1.5 & 1.1 & 1.9 & 1.5 & 1.5 & 1.2 & 1.7 & 1.0 & carbohydrate binding (IEA) \\
\hline
\end{tabular}


Appendix B. Continued.

\begin{tabular}{|c|c|c|c|c|c|c|c|c|c|c|}
\hline \multirow[b]{3}{*}{ CGD Name } & \multirow[b]{3}{*}{ orf19 Designation } & \multicolumn{8}{|c|}{ Change in fold expression ${ }^{a}$} & \multirow[b]{3}{*}{ Molecular function } \\
\hline & & \multicolumn{2}{|c|}{$\underline{\text { G648S }}$} & \multicolumn{2}{|c|}{$\underline{\text { G648D }}$} & \multicolumn{2}{|c|}{$\underline{\mathrm{Y} 642 \mathrm{~F}}$} & \multicolumn{2}{|c|}{$\underline{\mathrm{A} 643 \mathrm{~V}}$} & \\
\hline & & Exp1 & Exp2 & Exp1 & Exp2 & Exp1 & Exp2 & Exp1 & Exp2 & \\
\hline & orf19.91 & 1.2 & 0.9 & 1.5 & 1.5 & 1.2 & 1.0 & 1.5 & 1.3 & \\
\hline & orf19.6356 & 2.2 & 0.8 & 1.4 & 1.1 & 1.9 & 1.6 & 1.2 & 1.0 & RNA splicing factor activity, transesterification mechanism (IEA) \\
\hline$C M P 1$ & orf19.6033 & 1.4 & 1.2 & 1.2 & 1.8 & 1.7 & 1.5 & 1.1 & 1.3 & calcium-dependent protein serine/threonine phosphatase activity (ISS) \\
\hline PHO13 & orf19.1405 & 1.4 & 0.8 & 1.3 & 1.5 & 1.6 & 1.5 & 1.4 & 1.4 & 4-nitrophenylphosphatase activity (IEA)|alkaline phosphatase activity (IEA) \\
\hline$Y S T 1$ & orf19.6975 & 2.1 & 1.2 & 1.5 & 0.4 & 2.4 & 1.5 & 0.8 & 1.6 & structural constituent of ribosome (IGI) \\
\hline & orf19.6589 & 2.2 & 0.9 & 1.4 & 1.3 & 2.1 & 1.5 & 1.7 & 1.2 & rRNA binding (IEA) \\
\hline & orf19.5291 & 1.5 & 0.9 & 1.7 & 1.2 & 1.6 & 1.5 & 1.3 & 1.4 & \\
\hline FOX2 & orf19.1288 & 1.3 & 1.3 & 1.5 & 1.3 & 1.0 & 1.1 & 1.6 & 1.5 & nucleotide binding \\
\hline$A N T 1$ & orf19.6254 & 1.3 & 1.2 & 1.2 & 1.6 & 1.2 & 1.4 & 1.5 & 1.6 & adenine nucleotide transmembrane transporter activity (IEA) \\
\hline BMT8 & orf19.860 & 1.0 & 1.4 & 1.6 & 1.3 & 1.0 & 0.8 & 1.5 & 2.0 & \\
\hline$C D C 45$ & orf19.1988 & 1.6 & 1.2 & 1.7 & 1.3 & 1.6 & 1.0 & 1.5 & 1.5 & DNA replication origin binding (IEA)|chromatin binding (IEA) \\
\hline$D E F 1$ & orf19.7561 & 1.2 & 1.4 & 1.7 & 1.2 & 1.1 & 1.1 & 1.5 & 1.6 & \\
\hline GAL1 & orf19.3670 & 1.3 & 1.1 & 1.2 & 1.4 & 1.5 & 1.0 & 2.0 & 1.6 & galactokinase activity (IGI, NAS)|transcription activator activity (IEA) \\
\hline GAL10 & orf19.3672 & 1.0 & 1.3 & 1.1 & 1.5 & 1.3 & 1.1 & 1.6 & 1.6 & UDP-glucose 4-epimerase activity (IGI)|aldose 1-epimerase activity (IEA) \\
\hline HAP31 & orf19.517 & 1.3 & 1.0 & 1.3 & 1.8 & 1.5 & 1.2 & 1.7 & 1.5 & transcription factor activity (NAS)|transcription activator activity (IEA) \\
\hline$H G T 17$ & orf19.4682 & 0.7 & 0.8 & 1.5 & 1.2 & 0.8 & 0.9 & 2.3 & 2.2 & glucose transmembrane transporter activity (ISS) \\
\hline$H G T 20$ & orf19.1587 & 1.0 & 1.2 & 1.2 & 1.5 & 0.9 & 1.1 & 1.6 & 1.8 & glucose transmembrane transporter activity (ISS) \\
\hline HOS1 & orf19.4411 & 1.1 & 0.9 & 1.5 & 1.4 & 1.3 & 1.2 & 1.7 & 1.5 & histone deacetylase activity (ISS) \\
\hline INOI & orf19.7585 & 1.2 & 0.6 & 1.4 & 1.5 & 1.7 & 1.2 & 2.5 & 1.6 & inositol-3-phosphate synthase activity (IGI, ISS) \\
\hline PEX5 & orf19.5640 & 1.0 & 1.0 & 1.3 & 1.5 & 1.1 & 1.0 & 1.6 & 1.5 & peroxisome matrix targeting signal-1 binding (IEA)|protein binding, bridging (IEA) \\
\hline RBT5 & orf19.5636 & 1.3 & 1.5 & 1.2 & 1.8 & 1.4 & 1.8 & 1.8 & 2.0 & heme binding (IDA, IMP) \\
\hline SOD6 & orf19.2108 & 1.2 & 0.9 & 1.2 & 1.5 & 1.6 & 0.8 & 2.4 & 1.6 & superoxide dismutase activity (ISS) \\
\hline STF2 & orf19.2107.1 & 1.0 & 1.3 & 1.3 & 1.5 & 1.2 & 1.1 & 1.6 & 1.5 & \\
\hline SUR2 & orf19.5818 & 1.0 & 1.1 & 1.4 & 1.1 & 1.2 & 1.1 & 1.5 & 1.6 & sphingosine hydroxylase activity (IEA) \\
\hline
\end{tabular}


Appendix B. Continued.

\begin{tabular}{|c|c|c|c|c|c|c|c|c|c|c|}
\hline \multirow[b]{3}{*}{ CGD Name } & \multirow[b]{3}{*}{ orf19 Designation } & \multicolumn{8}{|c|}{ Change in fold expression ${ }^{a}$} & \multirow[b]{3}{*}{ Molecular function } \\
\hline & & \multicolumn{2}{|c|}{$\underline{\text { G648S }}$} & \multicolumn{2}{|c|}{$\underline{\text { G648D }}$} & \multicolumn{2}{|c|}{$\underline{\mathrm{Y} 642 \mathrm{~F}}$} & \multicolumn{2}{|c|}{$\underline{\mathrm{A} 643 \mathrm{~V}}$} & \\
\hline & & Exp1 & Exp2 & Exp1 & Exp2 & Exp1 & Exp2 & Exp1 & Exp2 & \\
\hline$V A M 3$ & orf19.5875 & 1.0 & 1.5 & 1.1 & 1.8 & 1.1 & 1.0 & 1.5 & 1.7 & \\
\hline ZCF11 & orf19.2423 & 1.0 & 1.2 & 1.3 & 1.9 & 0.9 & 1.2 & 1.5 & 2.0 & sequence-specific DNA binding (IEA) \\
\hline \multirow[t]{22}{*}{ ZCF14 } & orf19.2647 & 1.3 & 1.4 & 1.1 & 1.4 & 1.0 & 1.5 & 1.6 & 1.6 & transcription factor activity (ISS) \\
\hline & orf19.1412 & 1.4 & 1.1 & 1.4 & 1.5 & 1.4 & 1.0 & 1.8 & 1.5 & \\
\hline & orf19.1881 & 1.4 & 1.2 & 2.2 & 1.4 & 1.3 & 1.3 & 2.1 & 1.8 & 1-acylglycerol-3-phosphate O-acyltransferase activity (IEA) \\
\hline & orf19.1940 & 1.0 & 1.2 & 1.3 & 1.9 & 1.2 & 1.2 & 1.5 & 1.7 & \\
\hline & orf19.2286 & 1.4 & 1.2 & 1.6 & 1.4 & 1.5 & 1.3 & 1.6 & 1.5 & deoxyhypusine monooxygenase activity (IEA) \\
\hline & orf19.2313 & 1.4 & 1.6 & 1.4 & 2.3 & 0.9 & 0.8 & 1.7 & 1.8 & \\
\hline & orf19.314 & 1.1 & 1.2 & 1.5 & 1.4 & 1.4 & 1.3 & 1.6 & 1.6 & transcription repressor activity (IEA) \\
\hline & orf19.3434 & 1.6 & 1.1 & 1.4 & 1.3 & 1.6 & 1.3 & 2.1 & 1.6 & \\
\hline & orf19.3877 & 1.3 & 1.4 & 1.5 & 1.0 & 1.1 & 1.2 & 1.6 & 1.6 & \\
\hline & orf19.3937 & 1.4 & 1.6 & 1.1 & 1.4 & 1.4 & 0.9 & 1.8 & 1.6 & \\
\hline & orf19.4174 & 1.1 & 1.3 & 1.4 & 1.5 & 0.9 & 1.1 & 1.5 & 1.6 & nucleoside transmembrane transporter activity (IEA) \\
\hline & orf19.4504 & 1.4 & 1.3 & 1.5 & 1.4 & 1.2 & 0.8 & 2.1 & 1.7 & alcohol dehydrogenase (NAD) activity (IEA) \\
\hline & orf19.4595 & 1.0 & 1.3 & 1.3 & 1.3 & 1.1 & 0.9 & 1.5 & 1.5 & \\
\hline & orf19.4726 & 1.4 & 1.1 & 2.4 & 1.3 & 1.9 & 0.8 & 2.4 & 1.6 & calcium ion binding (IEA)|enzyme activator activity (IEA) \\
\hline & orf19.516 & 1.1 & 1.1 & 1.2 & 1.4 & 1.0 & 1.2 & 1.5 & 1.5 & \\
\hline & orf19.5237 & 1.2 & 1.2 & 1.4 & 1.7 & 1.6 & 1.1 & 1.7 & 1.8 & \\
\hline & orf19.5282 & 1.2 & 1.2 & 1.3 & 1.7 & 1.4 & 1.4 & 1.5 & 1.5 & \\
\hline & orf19.5319 & 1.7 & 1.4 & 1.6 & 1.1 & 2.1 & 0.9 & 1.7 & 1.6 & single-stranded DNA binding (IEA) \\
\hline & orf19.540 & 0.9 & 1.2 & 1.7 & 1.4 & 1.1 & 0.7 & 1.7 & 1.8 & \\
\hline & orf19.5523 & 1.0 & 1.0 & 1.2 & 1.2 & 1.3 & 0.9 & 1.6 & 1.5 & \\
\hline & orf19.6031 & 1.1 & 1.2 & 2.4 & 1.4 & 1.6 & 1.0 & 1.7 & 1.6 & phosphatidylinositol 3-phosphate binding (IEA)|protein binding (IEA) \\
\hline & orf19.6393 & 1.0 & 1.2 & 1.4 & 1.2 & 1.3 & 1.0 & 1.9 & 1.5 & ARF GTPase activator activity (IEA) \\
\hline
\end{tabular}


Appendix B. Continued.

\begin{tabular}{|c|c|c|c|c|c|c|c|c|c|c|}
\hline \multirow[b]{3}{*}{ CGD Name } & \multirow[b]{3}{*}{ orf19 Designation } & \multicolumn{8}{|c|}{ Change in fold expression ${ }^{a}$} & \multirow[b]{3}{*}{ Molecular function } \\
\hline & & \multicolumn{2}{|c|}{$\underline{\text { G648S }}$} & \multicolumn{2}{|c|}{$\underline{\text { G648D }}$} & \multicolumn{2}{|c|}{$\underline{\mathrm{Y} 642 \mathrm{~F}}$} & \multicolumn{2}{|c|}{$\underline{\mathrm{A} 643 \mathrm{~V}}$} & \\
\hline & & Exp1 & Exp2 & Exp1 & Exp2 & Exp1 & Exp2 & Exp1 & Exp2 & \\
\hline & orf19.6690 & 0.9 & 1.5 & 2.0 & 1.4 & 1.0 & 0.9 & 1.5 & 1.5 & \\
\hline & orf19.676 & 1.1 & 1.2 & 1.4 & 1.3 & 1.1 & 0.8 & 1.6 & 1.5 & structural constituent of cytoskeleton (IEA) \\
\hline & orf19.6816 & 1.0 & 1.0 & 1.3 & 1.6 & 1.2 & 1.1 & 1.6 & 1.5 & aldehyde reductase activity (IEA) \\
\hline & orf19.6852 & 1.0 & 1.1 & 1.4 & 1.6 & 1.3 & 1.2 & 1.6 & 1.5 & \\
\hline & orf19.6973 & 1.1 & 1.3 & 1.7 & 1.3 & 1.2 & 0.8 & 1.5 & 1.5 & ATP-dependent peptidase activity (IEA) \\
\hline & orf19.7444 & 1.2 & 1.6 & 1.3 & 1.2 & 2.0 & 0.9 & 2.4 & 1.6 & \\
\hline & orf19.7459 & 1.3 & 0.9 & 1.4 & 1.3 & 1.4 & 1.1 & 1.6 & 1.5 & \\
\hline & orf19.89 & 0.9 & 1.3 & 1.4 & 1.6 & 1.2 & 1.0 & 1.7 & 1.5 & peroxisome matrix targeting signal-2 binding (IEA) \\
\hline & orf19.897 & 1.1 & 1.2 & 1.5 & 1.4 & 1.1 & 1.0 & 1.8 & 1.5 & protein binding (IEA) \\
\hline & orf19.910 & 1.1 & 1.2 & 1.1 & 1.4 & 1.1 & 1.0 & 1.5 & 1.6 & \\
\hline$B N R I$ & orf19.7537 & 1.6 & 1.5 & 2.3 & 1.5 & 1.3 & 1.2 & 1.8 & 1.4 & cytoskeletal protein binding (IDA) \\
\hline$M U C 1$ & orf19.4183 & 1.5 & 1.5 & 2.5 & 1.5 & 1.5 & 1.1 & 1.7 & 1.3 & \\
\hline \multirow[t]{12}{*}{ USO6 } & orf19.6967 & 1.7 & 1.5 & 2.7 & 1.6 & 1.8 & 0.9 & 2.0 & 1.0 & \\
\hline & orf19.6148 & 1.5 & 17.0 & 3.5 & 19.0 & 1.9 & 7.7 & 0.9 & 5.5 & structural constituent of cytoskeleton (IEA) \\
\hline & orf19.6501 & 1.6 & 19.2 & 3.0 & 18.4 & 1.4 & 9.0 & 1.0 & 8.1 & \\
\hline & orf19.6790 & 1.5 & 1.5 & 2.8 & 1.5 & 1.6 & 0.7 & 1.3 & 0.9 & mRNA binding (IEA) \\
\hline & orf19.2400 & 1.5 & 1.9 & 1.6 & 2.0 & 1.8 & 1.3 & 1.4 & 1.4 & \\
\hline & orf19.3461 & 1.5 & 1.6 & 2.1 & 2.0 & 1.4 & 1.3 & 1.4 & 2.1 & \\
\hline & orf19.4330 & 1.7 & 2.0 & 2.3 & 1.9 & 1.7 & 1.5 & 1.4 & 1.8 & \\
\hline & orf19.6308 & 1.5 & 1.5 & 1.8 & 1.5 & 1.4 & 0.8 & 1.5 & 1.0 & \\
\hline & orf19.6920 & 2.0 & 1.8 & 2.7 & 2.1 & 1.6 & 1.4 & 1.5 & 0.9 & \\
\hline & orf19.2006 & 2.5 & 1.6 & 2.8 & 2.1 & 3.4 & 0.9 & 1.8 & 0.9 & \\
\hline & orf19.2204.2 & 1.5 & 1.5 & 2.1 & 1.8 & 1.7 & 1.4 & 1.8 & 1.4 & * GTPase activity \\
\hline & orf19.4168 & 1.6 & 1.7 & 1.6 & 1.7 & 2.3 & 1.4 & 1.9 & 1.1 & ribonuclease MRP activity (IEA) \\
\hline
\end{tabular}


Appendix B. Continued.

\begin{tabular}{|c|c|c|c|c|c|c|c|c|c|c|}
\hline \multirow[b]{3}{*}{ CGD Name } & \multirow[b]{3}{*}{ orf19 Designation } & \multicolumn{8}{|c|}{ Change in fold expression ${ }^{a}$} & \multirow[b]{3}{*}{ Molecular function } \\
\hline & & \multicolumn{2}{|c|}{$\underline{\text { G648S }}$} & \multicolumn{2}{|c|}{$\underline{\text { G648D }}$} & \multicolumn{2}{|c|}{$\underline{\mathrm{Y} 642 \mathrm{~F}}$} & \multicolumn{2}{|c|}{$\underline{\mathrm{A} 643 \mathrm{~V}}$} & \\
\hline & & Exp1 & Exp2 & Exp1 & Exp2 & Exp1 & Exp2 & Exp1 & Exp2 & \\
\hline & orf19.5772 & 1.8 & 1.5 & 1.7 & 1.6 & 1.9 & 1.2 & 1.9 & 1.1 & \\
\hline & orf19.5295 & 1.6 & 2.1 & 2.5 & 1.6 & 0.9 & 0.9 & 1.9 & 1.3 & \\
\hline & orf19.641 & 1.8 & 1.5 & 2.2 & 1.6 & 1.7 & 1.3 & 2.0 & 1.3 & \\
\hline & orf19.1297 & 2.2 & 1.6 & 2.5 & 1.8 & 1.3 & 1.5 & 2.2 & 1.4 & \\
\hline & orf19.5426 & 1.7 & 1.9 & 2.6 & 1.9 & 1.5 & 0.9 & 2.3 & 1.4 & triglyceride lipase activity (IEA) \\
\hline & orf19.3437 & 2.5 & 2.3 & 2.5 & 1.9 & 0.2 & 1.0 & 2.3 & 1.3 & \\
\hline & orf19.1444 & 2.0 & 1.7 & 4.4 & 1.9 & 2.9 & 1.6 & 2.6 & 1.4 & phosphatidylinositol-4,5-bisphosphate binding \\
\hline & orf19.3607 & 3.8 & 10.1 & 3.2 & 15.1 & 1.4 & 6.4 & 0.4 & 13.9 & \\
\hline ARG81 & orf19.4766 & 1.7 & 1.5 & 1.9 & 1.6 & 2.0 & 1.0 & 1.7 & 1.2 & transcription factor activity (ISS)|transcription cofactor activity (IEA) \\
\hline $\mathrm{BIO} 2$ & orf19.2593 & 1.6 & 1.8 & 3.0 & 1.6 & 1.3 & 1.0 & 1.5 & 1.3 & biotin synthase activity (NAS) \\
\hline$B N R 1$ & orf19.7537 & 1.6 & 1.5 & 2.3 & 1.5 & 1.3 & 1.2 & 1.8 & 1.4 & cytoskeletal protein binding (IDA)|cytoskeletal regulatory protein binding (IEA) \\
\hline USO6 & orf19.6967 & 1.7 & 1.5 & 2.7 & 1.6 & 1.8 & 0.9 & 2.0 & 1.0 & \\
\hline & orf19.6501 & 1.6 & 19.2 & 3.0 & 18.4 & 1.4 & 9.0 & 1.0 & 8.1 & \\
\hline & orf19.3461 & 1.5 & 1.6 & 2.1 & 2.0 & 1.4 & 1.3 & 1.4 & 2.1 & \\
\hline & orf19.2400 & 1.5 & 1.9 & 1.6 & 2.0 & 1.8 & 1.3 & 1.4 & 1.4 & \\
\hline & orf19.5295 & 1.6 & 2.1 & 2.5 & 1.6 & 0.9 & 0.9 & 1.9 & 1.3 & \\
\hline & orf19.641 & 1.8 & 1.5 & 2.2 & 1.6 & 1.7 & 1.3 & 2.0 & 1.3 & \\
\hline & orf19.5772 & 1.8 & 1.5 & 1.7 & 1.6 & 1.9 & 1.2 & 1.9 & 1.1 & \\
\hline & orf19.4168 & 1.6 & 1.7 & 1.6 & 1.7 & 2.3 & 1.4 & 1.9 & 1.1 & ribonuclease MRP activity (IEA) \\
\hline & orf19.3998 & 1.5 & 1.6 & 3.7 & 1.6 & 2.0 & 0.6 & 1.9 & 1.1 & \\
\hline & orf19.6308 & 1.5 & 1.5 & 1.8 & 1.5 & 1.4 & 0.8 & 1.5 & 1.0 & \\
\hline & orf19.2006 & 2.5 & 1.6 & 2.8 & 2.1 & 3.4 & 0.9 & 1.8 & 0.9 & \\
\hline & orf19.6920 & 2.0 & 1.8 & 2.7 & 2.1 & 1.6 & 1.4 & 1.5 & 0.9 & \\
\hline & orf19.6790 & 1.5 & 1.5 & 2.8 & 1.5 & 1.6 & 0.7 & 1.3 & 0.9 & mRNA binding (IEA) \\
\hline
\end{tabular}


Appendix B. Continued.

\begin{tabular}{|c|c|c|c|c|c|c|c|c|c|c|}
\hline \multirow[b]{3}{*}{ CGD Name } & \multirow[b]{3}{*}{ orf19 Designation } & \multicolumn{8}{|c|}{ Change in fold expression ${ }^{a}$} & \multirow[b]{3}{*}{ Molecular function } \\
\hline & & \multicolumn{2}{|c|}{ G648S } & \multicolumn{2}{|c|}{$\underline{\text { G648D }}$} & \multicolumn{2}{|c|}{$\underline{\mathrm{Y} 642 \mathrm{~F}}$} & \multicolumn{2}{|c|}{$\underline{\mathrm{A} 643 \mathrm{~V}}$} & \\
\hline & & Exp1 & Exp2 & Exp1 & Exp2 & Exp1 & Exp2 & Exp1 & Exp2 & \\
\hline \multirow[t]{2}{*}{$R B T 1$} & orf19.1327 & 1.9 & 1.6 & 1.2 & 1.1 & 1.6 & 1.9 & 1.1 & 1.2 & \\
\hline & orf19.3851 & 1.5 & 1.6 & 2.1 & 1.1 & 0.9 & 0.9 & 1.6 & 1.6 & \\
\hline \multirow[t]{2}{*}{ ALD6 } & orf19.742 & 2.6 & 3.2 & 1.4 & 1.2 & 1.7 & 0.7 & 1.8 & 1.5 & aldehyde dehydrogenase (NAD) activity (IEA) \\
\hline & orf19.136 & 1.6 & 1.5 & 1.3 & 1.3 & 1.0 & 1.1 & 1.6 & 1.5 & drug transporter activity (ISS)|multidrug efflux pump activity (IEA) \\
\hline CIRT4B & orf19.2839 & 1.9 & 1.7 & 2.9 & 1.3 & 1.4 & 0.5 & 3.4 & 1.5 & \\
\hline$C A G 1$ & orf19.4015 & 1.2 & 2.1 & 1.7 & 1.5 & 1.7 & 1.7 & 1.2 & 1.4 & GTPase activity (IGI)|guanyl nucleotide binding (ISS) \\
\hline \multirow[t]{6}{*}{$F G R 23$} & orf19.1616 & 0.7 & 2.5 & 1.5 & 2.4 & 1.7 & 1.5 & 1.2 & 2.3 & \\
\hline & orf19.345 & 2.1 & 1.4 & 1.6 & 1.8 & 2.3 & 1.5 & 1.9 & 1.3 & succinate-semialdehyde dehydrogenase $[\mathrm{NAD}(\mathrm{P})+]$ activity $(\mathrm{IEA})$ \\
\hline & orf19.6805 & 1.8 & 1.3 & 2.8 & 1.6 & 2.1 & 1.5 & 1.5 & 1.0 & \\
\hline & orf19.2650.1 & 1.4 & 1.2 & 1.7 & 1.6 & 1.8 & 1.6 & 1.3 & 1.1 & structural constituent of ribosome (IEA) \\
\hline & orf19.6023 & 1.3 & 1.6 & 2.2 & 1.5 & 1.7 & 1.8 & 1.3 & 1.5 & drug transmembrane transport \\
\hline & orf19.6311 & 1.0 & 4.2 & 3.9 & 4.5 & 2.1 & 1.7 & 1.4 & 1.2 & \\
\hline$H G T 20$ & orf19.1587 & 1.3 & 1.1 & 1.5 & 1.6 & 1.0 & 1.0 & 1.8 & 1.8 & glucose transmembrane transporter activity (ISS) \\
\hline HGT3 & orf19.4356 & 1.1 & 1.5 & 2.5 & 1.7 & 1.0 & 1.0 & 1.9 & 1.7 & glucose transmembrane transporter activity (ISS) \\
\hline HYRl & orf19.4975 & 1.3 & 1.3 & 1.7 & 1.5 & 1.2 & 1.0 & 1.8 & 1.5 & \\
\hline$I F C 3$ & orf19.3749 & 1.1 & 1.7 & 5.9 & 3.6 & 1.3 & 1.2 & 3.6 & 3.4 & oligopeptide transmembrane transporter activity \\
\hline$I H D 1$ & orf19.5760 & 1.3 & 1.8 & 1.7 & 1.9 & 1.2 & 1.7 & 1.7 & 2.0 & \\
\hline IRS4 & orf19.6953 & 1.3 & 1.4 & 1.9 & 1.6 & 1.5 & 1.3 & 1.7 & 1.5 & \\
\hline$L A P 3$ & orf19.539 & 1.1 & 2.0 & 2.6 & 2.0 & 1.2 & 1.0 & 1.8 & 2.0 & aminopeptidase activity (NAS) \\
\hline$M E P 1$ & orf19.1614 & 1.1 & 2.2 & 6.6 & 4.5 & 1.2 & 1.1 & 2.9 & 3.5 & ammonium transmembrane transporter activity (IGI) \\
\hline MET13 & orf19.2887 & 1.4 & 1.3 & 1.8 & 1.6 & 1.3 & 1.3 & 1.6 & 1.5 & \\
\hline MSO1 & orf19.5248 & 1.3 & 1.3 & 3.0 & 2.9 & 2.1 & 1.3 & 2.9 & 2.3 & \\
\hline ORC3 & orf19.6942 & 1.4 & 1.2 & 1.8 & 1.6 & 1.4 & 1.2 & 1.8 & 1.6 & DNA replication origin binding (IEA)|chromatin binding (IEA) \\
\hline PGA10 & orf19.5674 & 1.3 & 2.2 & 2.1 & 2.8 & 1.4 & 2.1 & 1.7 & 2.6 & heme binding (IDA, IGI) \\
\hline
\end{tabular}


Appendix B. Continued.

\begin{tabular}{|c|c|c|c|c|c|c|c|c|c|c|}
\hline \multirow[b]{3}{*}{ CGD Name } & \multirow[b]{3}{*}{ orf19 Designation } & \multicolumn{8}{|c|}{ Change in fold expression ${ }^{\mathrm{a}}$} & \multirow[b]{3}{*}{ Molecular function } \\
\hline & & \multicolumn{2}{|c|}{$\underline{\text { G648S }}$} & \multicolumn{2}{|c|}{$\underline{\text { G648D }}$} & \multicolumn{2}{|c|}{$\underline{\mathrm{Y} 642 \mathrm{~F}}$} & \multicolumn{2}{|c|}{$\underline{\mathrm{A} 643 \mathrm{~V}}$} & \\
\hline & & Exp1 & Exp2 & Exp1 & Exp2 & Exp1 & Exp2 & Exp1 & Exp2 & \\
\hline PLB4 & orf19.1443 & 1.4 & 1.9 & 2.0 & 1.9 & 1.2 & 1.3 & 1.5 & 1.5 & lysophospholipase activity (ISS) \\
\hline POS5 & orf19.6800 & 1.1 & 1.5 & 2.2 & 2.1 & 1.4 & 0.9 & 1.9 & 1.9 & NADH kinase activity (IEA) \\
\hline PTC4 & orf19.6638 & 0.9 & 1.1 & 1.6 & 1.7 & 1.2 & 1.0 & 1.5 & 1.5 & protein serine/threonine phosphatase activity (IEA) \\
\hline PTC6 & orf19.3705 & 1.7 & 1.3 & 2.1 & 2.1 & 1.7 & 1.2 & 2.3 & 1.9 & protein serine/threonine phosphatase activity (IDA) \\
\hline RAD57 & orf19.2174 & 1.3 & 1.3 & 2.3 & 1.7 & 1.6 & 1.1 & 1.9 & 1.5 & protein heterodimerization activity (IEA) \\
\hline$R P N 4$ & orf19.1069 & 1.4 & 1.1 & 2.0 & 2.2 & 1.5 & 1.3 & 1.7 & 1.6 & sequence-specific DNA binding (IDA)|transcription factor activity (ISS) \\
\hline SAP5 & orf19.5585 & 1.0 & 2.3 & 1.9 & 2.9 & 0.9 & 0.8 & 1.9 & 2.8 & aspartic-type endopeptidase activity (IDA, ISS)|drug binding (IDA,ISS) \\
\hline$S C T 2$ & orf19.5815 & 1.5 & 1.1 & 2.5 & 1.7 & 1.3 & 1.2 & 1.8 & 1.5 & \\
\hline SOD6 & orf19.2108 & 1.9 & 1.2 & 1.9 & 2.1 & 1.6 & 1.1 & 2.6 & 1.7 & superoxide dismutase activity (ISS) \\
\hline$U B A 4$ & orf19.2324 & 1.5 & 1.0 & 1.5 & 1.7 & 1.6 & 1.1 & 1.9 & 1.6 & URM1 activating enzyme activity (IEA) \\
\hline$V A M 3$ & orf19.5875 & 0.9 & 1.5 & 1.6 & 1.7 & 1.5 & 1.0 & 1.5 & 2.0 & \\
\hline VPS51 & orf19.5568 & 1.2 & 1.3 & 2.0 & 1.7 & 1.9 & 1.0 & 2.0 & 1.5 & \\
\hline$V R P 1$ & orf19.2190 & 1.0 & 2.3 & 2.2 & 2.4 & 1.2 & 1.2 & 1.6 & 2.1 & actin binding (IEA) \\
\hline YРT31 & orf19.2622 & 1.5 & 1.2 & 2.0 & 1.6 & 1.7 & 1.4 & 1.9 & 1.5 & GTPase activity (IEA) \\
\hline \multirow[t]{10}{*}{ ZCF21 } & orf19.4166 & 1.8 & 1.2 & 2.2 & 1.9 & 1.6 & 1.3 & 2.1 & 1.6 & \\
\hline & orf19.90 & 1.3 & 1.1 & 4.9 & 3.4 & 1.9 & 1.1 & 3.9 & 2.3 & \\
\hline & orf19.2515 & 1.4 & 4.0 & 9.4 & 4.5 & 1.8 & 1.3 & 3.6 & 3.2 & \\
\hline & orf19.5205 & 1.3 & 1.6 & 2.5 & 2.7 & 1.5 & 0.9 & 3.4 & 2.5 & \\
\hline & orf19.4031 & 1.6 & 1.4 & 3.3 & 2.4 & 2.0 & 1.2 & 3.4 & 2.2 & \\
\hline & orf19.6027 & 1.3 & 1.3 & 1.5 & 1.6 & 2.1 & 1.0 & 3.3 & 1.5 & \\
\hline & orf19.7578 & 1.2 & 1.7 & 2.7 & 2.2 & 1.5 & 1.0 & 3.1 & 2.3 & protein transporter activity (IEA) \\
\hline & orf19.7457 & 1.4 & 1.6 & 2.3 & 2.7 & 1.7 & 1.2 & 2.7 & 1.8 & \\
\hline & orf19.4897 & 1.9 & 1.3 & 2.7 & 2.2 & 2.3 & 1.4 & 2.7 & 1.8 & phosphatidylinositol transporter activity (IEA) \\
\hline & orf19.3318 & 1.5 & 1.2 & 3.1 & 2.0 & 1.6 & 1.0 & 2.6 & 1.6 & \\
\hline
\end{tabular}


Appendix B. Continued.

\begin{tabular}{|c|c|c|c|c|c|c|c|c|c|c|}
\hline \multirow[b]{3}{*}{ CGD Name } & \multirow[b]{3}{*}{ orf19 Designation } & \multicolumn{8}{|c|}{ Change in fold expression ${ }^{\mathrm{a}}$} & \multirow[b]{3}{*}{ Molecular function } \\
\hline & & \multicolumn{2}{|c|}{$\underline{\text { G648S }}$} & \multicolumn{2}{|c|}{$\underline{\text { G648D }}$} & \multicolumn{2}{|c|}{$\underline{\mathrm{Y} 642 \mathrm{~F}}$} & \multicolumn{2}{|c|}{$\underline{\mathrm{A} 643 \mathrm{~V}}$} & \\
\hline & & Exp1 & Exp2 & Exp1 & Exp2 & Exp1 & Exp2 & Exp1 & Exp2 & \\
\hline & orf19.5777 & 1.2 & 2.1 & 3.5 & 3.6 & 1.3 & 1.6 & 2.6 & 2.9 & \\
\hline & orf19.5535 & 1.4 & 1.7 & 2.2 & 2.6 & 1.4 & 1.9 & 2.5 & 2.9 & anion:cation symporter activity (ISS)|pantothenate transporter activity (IEA) \\
\hline & orf19.5727 & 1.2 & 1.5 & 2.7 & 2.4 & 1.4 & 1.2 & 2.5 & 2.0 & \\
\hline & orf19.3544 & 1.1 & 1.9 & 3.5 & 1.6 & 1.1 & 1.0 & 2.4 & 1.9 & \\
\hline & orf19.5275 & 1.4 & 1.3 & 3.0 & 2.2 & 2.7 & 1.4 & 2.4 & 1.8 & ATPase activator activity (IEA) \\
\hline & orf19.951 & 1.3 & 1.4 & 2.1 & 2.6 & 1.8 & 1.1 & 2.4 & 1.5 & \\
\hline & orf19.288 & 1.6 & 1.2 & 2.7 & 2.4 & 1.7 & 1.4 & 2.4 & 1.8 & methylenetetrahydrofolate reductase (NADPH) activity (IEA) \\
\hline & orf19.1985 & 1.6 & 1.4 & 2.4 & 2.1 & 1.6 & 1.3 & 2.3 & 2.2 & * protein phosphorylation \\
\hline & orf19.4287 & 1.1 & 2.1 & 3.2 & 3.7 & 1.1 & 1.3 & 2.3 & 3.0 & $(\mathrm{R}, \mathrm{R})$-butanediol dehydrogenase activity (IEA) \\
\hline & orf19.910 & 1.7 & 1.2 & 1.7 & 1.7 & 1.9 & 1.3 & 2.3 & 1.7 & \\
\hline & orf19.1277 & 1.2 & 2.1 & 2.2 & 2.3 & 1.5 & 1.3 & 2.3 & 1.9 & \\
\hline & orf19.2943 & 1.5 & 1.0 & 2.4 & 2.3 & 1.7 & 0.9 & 2.3 & 1.7 & amino acid transmembrane transporter activity (IEA) \\
\hline & orf19.1592 & 1.9 & 1.2 & 2.1 & 1.6 & 2.0 & 1.3 & 2.2 & 1.5 & \\
\hline & orf19.996 & 1.3 & 1.3 & 2.5 & 1.9 & 1.9 & 1.1 & 2.2 & 1.7 & adenylate cyclase activity (IEA) \\
\hline & orf19.1698 & 1.7 & 1.3 & 2.3 & 1.5 & 1.6 & 1.0 & 2.2 & 1.7 & \\
\hline & orf19.4723 & 1.5 & 1.2 & 1.7 & 1.6 & 1.4 & 1.2 & 2.2 & 1.5 & FMN adenylyltransferase activity (IEA) \\
\hline & orf19.2691 & 1.0 & 2.8 & 3.8 & 4.1 & 0.9 & 1.3 & 2.2 & 3.2 & \\
\hline & orf19.7347 & 1.5 & 1.1 & 1.9 & 1.6 & 1.6 & 1.2 & 2.1 & 1.7 & ubiquitin-protein ligase activity (ISS) \\
\hline & orf19.35 & 1.3 & 1.1 & 2.1 & 1.9 & 1.4 & 1.1 & 2.1 & 1.6 & \\
\hline & orf19.89 & 1.2 & 1.0 & 1.6 & 1.9 & 1.3 & 1.0 & 2.1 & 1.6 & peroxisome matrix targeting signal-2 binding (IEA) \\
\hline & orf19.4711 & 1.2 & 1.3 & 2.1 & 2.1 & 1.4 & 1.2 & 2.1 & 1.8 & \\
\hline & orf19.3135 & 1.3 & 1.2 & 2.0 & 1.8 & 1.3 & 1.0 & 2.1 & 1.7 & protein binding, bridging (IEA) \\
\hline & orf19.296 & 0.9 & 2.4 & 3.4 & 2.3 & 0.9 & 1.0 & 2.1 & 2.2 & \\
\hline & orf19.6912 & 1.4 & 1.1 & 2.5 & 1.6 & 1.5 & 1.1 & 2.1 & 1.7 & choline kinase activity (IEA) \\
\hline
\end{tabular}


Appendix B. Continued.

\begin{tabular}{|c|c|c|c|c|c|c|c|c|c|c|}
\hline \multirow[b]{3}{*}{ CGD Name } & \multirow[b]{3}{*}{ orf19 Designation } & \multicolumn{8}{|c|}{ Change in fold expression ${ }^{\mathrm{a}}$} & \multirow[b]{3}{*}{ Molecular function } \\
\hline & & \multicolumn{2}{|c|}{$\underline{\text { G648S }}$} & \multicolumn{2}{|c|}{$\underline{\text { G648D }}$} & \multicolumn{2}{|c|}{$\underline{\mathrm{Y} 642 \mathrm{~F}}$} & \multicolumn{2}{|c|}{$\underline{\mathrm{A} 643 \mathrm{~V}}$} & \\
\hline & & Exp1 & Exp2 & Exp1 & Exp2 & Exp1 & Exp2 & Exp1 & Exp2 & \\
\hline & orf19.1857 & 1.7 & 1.2 & 2.2 & 1.6 & 1.9 & 1.0 & 2.1 & 1.5 & histone acetyltransferase activity (IEA) \\
\hline & orf19.2026 & 1.2 & 1.1 & 1.7 & 1.7 & 1.5 & 1.0 & 2.1 & 1.6 & ubiquitin-specific protease activity (IEA) \\
\hline & orf19.2703 & 1.2 & 1.1 & 2.1 & 1.7 & 1.1 & 1.1 & 2.1 & 1.6 & \\
\hline & orf19.6624 & 1.6 & 1.3 & 1.9 & 1.8 & 1.2 & 1.2 & 2.1 & 1.5 & \\
\hline & orf19.5601 & 1.2 & 1.5 & 2.9 & 2.4 & 1.4 & 1.0 & 2.1 & 1.7 & \\
\hline & orf19.5492 & 1.2 & 1.0 & 2.0 & 1.8 & 1.4 & 1.2 & 2.1 & 1.5 & mRNA binding (IEA) \\
\hline & orf19.7131 & 1.4 & 1.8 & 2.9 & 1.9 & 1.3 & 1.3 & 2.0 & 1.6 & gamma-butyrobetaine dioxygenase activity (IMP) \\
\hline & orf19.6557 & 1.8 & 1.4 & 2.4 & 1.5 & 1.7 & 1.1 & 2.0 & 1.9 & \\
\hline & orf19.7567 & 1.4 & 1.8 & 3.7 & 2.7 & 1.7 & 1.2 & 2.0 & 2.0 & \\
\hline & orf19.3881 & 1.2 & 1.3 & 2.2 & 2.0 & 1.2 & 1.0 & 2.0 & 1.7 & \\
\hline & orf19.5249 & 1.2 & 1.4 & 2.6 & 1.9 & 1.6 & 1.1 & 2.0 & 1.5 & \\
\hline & orf19.3734 & 1.2 & 1.3 & 2.0 & 1.9 & 1.4 & 1.1 & 2.0 & 1.7 & \\
\hline & orf19.5114 & 1.0 & 1.4 & 2.0 & 2.2 & 1.1 & 1.1 & 2.0 & 1.6 & phosphatidylinositol 3-phosphate binding (IEA)|protein binding (IEA) \\
\hline & orf19.4658 & 1.6 & 1.4 & 3.3 & 2.6 & 1.7 & 1.3 & 2.0 & 1.5 & \\
\hline & orf19.6057 & 1.4 & 1.3 & 1.9 & 2.2 & 1.5 & 1.4 & 2.0 & 1.9 & 3-methyl-2-oxobutanoate hydroxymethyltransferase activity (IEA) \\
\hline & orf19.1162.1 & 1.1 & 1.3 & 2.4 & 1.6 & 1.3 & 1.1 & 1.9 & 1.7 & \\
\hline & orf19.719 & 1.1 & 1.5 & 1.7 & 2.4 & 1.3 & 1.2 & 1.9 & 1.8 & ubiquitin-protein ligase activity (IEA) \\
\hline & orf19.6148 & 1.4 & 1.9 & 4.5 & 3.0 & 1.8 & 1.4 & 1.9 & 1.8 & structural constituent of cytoskeleton (IEA) \\
\hline & orf19.6626 & 1.2 & 1.3 & 1.9 & 2.2 & 1.4 & 1.4 & 1.9 & 1.8 & \\
\hline & orf19.6411 & 1.3 & 1.4 & 2.4 & 2.3 & 1.3 & 1.3 & 1.9 & 1.8 & enzyme activator activity (IEA) \\
\hline & orf19.4292 & 1.2 & 1.1 & 1.8 & 1.8 & 1.4 & 1.1 & 1.9 & 1.5 & SNAP receptor activity (IEA) \\
\hline & orf19.6832 & 1.2 & 1.3 & 1.8 & 1.8 & 1.4 & 1.1 & 1.9 & 1.6 & \\
\hline & orf19.1306 & 1.2 & 0.9 & 2.2 & 2.1 & 1.4 & 1.3 & 1.9 & 1.8 & \\
\hline & orf19.775 & 1.1 & 1.6 & 2.4 & 2.1 & 1.3 & 1.1 & 1.8 & 1.6 & \\
\hline
\end{tabular}


Appendix B. Continued.

\begin{tabular}{|c|c|c|c|c|c|c|c|c|c|c|}
\hline \multirow[b]{3}{*}{ CGD Name } & \multirow[b]{3}{*}{ orf19 Designation } & \multicolumn{8}{|c|}{ Change in fold expression ${ }^{\mathrm{a}}$} & \multirow[b]{3}{*}{ Molecular function } \\
\hline & & \multicolumn{2}{|c|}{$\underline{\text { G648S }}$} & \multicolumn{2}{|c|}{$\underline{\text { G648D }}$} & \multicolumn{2}{|c|}{$\underline{\mathrm{Y} 642 \mathrm{~F}}$} & \multicolumn{2}{|c|}{$\underline{\mathrm{A} 643 \mathrm{~V}}$} & \\
\hline & & Exp1 & Exp2 & Exp1 & $\operatorname{Exp2}$ & Exp1 & Exp2 & Exp1 & Exp2 & \\
\hline & orf19.2278 & 1.4 & 1.6 & 2.5 & 2.2 & 1.6 & 1.4 & 1.8 & 1.7 & \\
\hline & orf19.5125 & 1.1 & 1.8 & 2.4 & 1.6 & 1.1 & 0.8 & 1.8 & 1.5 & \\
\hline & orf19.5711 & 1.4 & 1.4 & 2.1 & 1.5 & 1.4 & 1.2 & 1.8 & 1.7 & phosphatidylinositol transporter activity (IEA) \\
\hline & orf19.1424 & 1.1 & 1.6 & 2.1 & 2.2 & 1.1 & 1.5 & 1.8 & 1.9 & oxalate transmembrane transporter activity (ISS) \\
\hline & orf19.4699 & 1.0 & 2.2 & 2.1 & 2.5 & 1.2 & 1.3 & 1.8 & 2.0 & phospholipase activity (ISS)|triglyceride lipase activity (IEA) \\
\hline & orf19.5925 & 1.3 & 1.1 & 1.5 & 1.6 & 1.7 & 1.0 & 1.8 & 1.8 & \\
\hline & orf19.577 & 1.2 & 1.4 & 2.3 & 1.8 & 1.2 & 1.1 & 1.8 & 1.7 & \\
\hline & orf19.6194 & 1.1 & 1.5 & 1.8 & 1.7 & 1.3 & 1.1 & 1.8 & 1.5 & \\
\hline & orf19.5459 & 1.3 & 2.0 & 1.9 & 1.6 & 1.2 & 1.8 & 1.7 & 1.8 & \\
\hline & orf19.7092 & 1.3 & 1.2 & 2.0 & 1.7 & 1.4 & 1.1 & 1.7 & 1.5 & \\
\hline & orf19.346 & 1.2 & 1.6 & 2.0 & 1.8 & 1.1 & 1.0 & 1.7 & 1.6 & L-alanine:2-oxoglutarate aminotransferase activity (IEA) \\
\hline & orf19.5751 & 1.2 & 1.2 & 2.0 & 2.4 & 1.4 & 1.3 & 1.7 & 1.5 & \\
\hline & orf19.3058 & 1.3 & 1.4 & 1.9 & 2.0 & 1.3 & 1.3 & 1.7 & 1.9 & \\
\hline & orf19.6816 & 1.0 & 1.1 & 1.5 & 1.7 & 1.2 & 1.1 & 1.7 & 1.6 & aldehyde reductase activity (IEA) \\
\hline & orf19.5752 & 1.1 & 1.3 & 1.8 & 2.0 & 1.2 & 1.2 & 1.7 & 1.7 & contributes_to ubiquitin-protein ligase activity (IEA) \\
\hline & orf19.6043 & 1.3 & 1.5 & 2.5 & 1.9 & 1.7 & 1.3 & 1.7 & 1.8 & D-lactate dehydrogenase (cytochrome) activity (IEA) \\
\hline & orf19.2558 & 1.3 & 1.3 & 1.5 & 2.1 & 1.4 & 1.6 & 1.7 & 1.9 & \\
\hline & orf19.2050 & 0.9 & 1.6 & 1.9 & 1.9 & 1.0 & 1.1 & 1.7 & 2.0 & sterol esterase activity (IEA) \\
\hline & orf19.3615 & 1.3 & 1.6 & 2.3 & 2.9 & 1.5 & 1.3 & 1.7 & 1.9 & \\
\hline & orf19.3991 & 1.3 & 1.2 & 1.7 & 1.6 & 1.3 & 1.3 & 1.7 & 1.5 & \\
\hline & orf19.6850 & 1.1 & 1.3 & 1.7 & 1.7 & 1.2 & 1.1 & 1.7 & 1.7 & transcription factor activity (ISS)|ubiquitin-protein ligase activity (IEA) \\
\hline & orf19.3508 & 0.8 & 2.0 & 2.2 & 2.0 & 1.0 & 1.2 & 1.7 & 2.3 & \\
\hline & orf19.135 & 1.4 & 1.2 & 2.1 & 1.6 & 1.7 & 1.0 & 1.7 & 1.5 & protein binding (IEA) \\
\hline & orf19.3815 & 1.4 & 1.1 & 1.5 & 1.8 & 1.4 & 1.0 & 1.7 & 1.6 & ubiquitin-specific protease activity (IEA) \\
\hline
\end{tabular}


Appendix B. Continued.

\begin{tabular}{|c|c|c|c|c|c|c|c|c|c|c|}
\hline \multirow[b]{3}{*}{ CGD Name } & \multirow[b]{3}{*}{ orf19 Designation } & \multicolumn{8}{|c|}{ Change in fold expression ${ }^{\mathrm{a}}$} & \multirow[b]{3}{*}{ Molecular function } \\
\hline & & \multicolumn{2}{|c|}{$\underline{\text { G648S }}$} & \multicolumn{2}{|c|}{ G648D } & \multicolumn{2}{|c|}{$\underline{\mathrm{Y} 642 \mathrm{~F}}$} & \multicolumn{2}{|c|}{$\underline{\mathrm{A} 643 \mathrm{~V}}$} & \\
\hline & & Exp1 & Exp2 & Exp1 & Exp2 & Exp1 & Exp2 & Exp1 & Exp2 & \\
\hline & orf19.1630 & 1.5 & 1.1 & 1.6 & 1.6 & 1.3 & 1.3 & 1.7 & 1.5 & \\
\hline & orf19.3910 & 0.9 & 2.2 & 2.9 & 2.4 & 0.8 & 0.6 & 1.7 & 1.7 & \\
\hline & orf19.1433 & 1.1 & 1.4 & 1.8 & 2.0 & 1.3 & 1.1 & 1.6 & 1.6 & \\
\hline & orf19.6102 & 1.2 & 1.4 & 1.9 & 1.8 & 1.3 & 1.3 & 1.6 & 1.8 & specific RNA polymerase II transcription factor activity (IEA) \\
\hline & orf19.467 & 1.3 & 2.0 & 2.8 & 1.7 & 1.3 & 1.1 & 1.6 & 1.6 & \\
\hline & orf19.6860 & 1.2 & 1.4 & 1.6 & 1.8 & 1.2 & 1.3 & 1.6 & 1.6 & CDP-diacylglycerol-inositol 3-phosphatidyltransferase activity (IEA) \\
\hline & orf19.1307 & 1.0 & 2.9 & 3.2 & 1.7 & 0.8 & 1.2 & 1.6 & 2.1 & \\
\hline & orf19.6838 & 1.1 & 1.8 & 1.9 & 2.0 & 1.1 & 1.3 & 1.6 & 2.0 & 2,4-dienoyl-CoA reductase (NADPH) activity (IEA) \\
\hline & orf19.1162 & 1.2 & 1.5 & 1.7 & 1.6 & 1.3 & 1.4 & 1.6 & 1.7 & \\
\hline & orf19.1940 & 1.0 & 1.4 & 1.6 & 2.1 & 1.2 & 1.3 & 1.6 & 1.8 & \\
\hline & orf19.4865 & 1.2 & 1.2 & 1.5 & 1.6 & 1.2 & 1.3 & 1.6 & 1.7 & phosphatidylinositol-3,5-bisphosphate 5-phosphatase activity (IEA) \\
\hline & orf19.6003 & 1.6 & 1.3 & 2.2 & 1.7 & 1.6 & 0.8 & 1.6 & 1.5 & \\
\hline & orf19.3659 & 1.1 & 1.2 & 1.6 & 1.7 & 1.3 & 1.2 & 1.6 & 1.6 & \\
\hline & orf19.5614 & 0.9 & 2.6 & 2.4 & 3.1 & 0.9 & 2.0 & 1.6 & 2.3 & ribonuclease $\mathrm{H}$ activity (IEA) \\
\hline & orf19.7459 & 1.2 & 0.9 & 1.5 & 1.5 & 1.3 & 1.2 & 1.6 & 1.6 & \\
\hline & orf19.2838 & 0.7 & 1.5 & 1.5 & 1.6 & 0.8 & 0.8 & 1.6 & 1.9 & \\
\hline & orf19.2163 & 1.0 & 1.7 & 1.8 & 1.9 & 1.1 & 1.2 & 1.6 & 1.6 & \\
\hline & orf19.417 & 1.4 & 1.4 & 1.6 & 1.8 & 1.3 & 1.5 & 1.6 & 1.6 & metallochaperone activity (IEA)|transporter activity (IEA) \\
\hline & orf19.3973 & 0.9 & 2.0 & 2.1 & 2.1 & 1.0 & 1.1 & 1.6 & 2.0 & \\
\hline & orf19.5953 & 1.4 & 1.2 & 1.5 & 1.9 & 1.3 & 1.4 & 1.5 & 1.7 & sequence-specific DNA binding (IEA)|transcription factor activity (IEA) \\
\hline & orf19.4127 & 1.1 & 1.2 & 1.5 & 1.7 & 1.3 & 1.2 & 1.5 & 1.5 & \\
\hline & orf19.4055 & 0.7 & 1.1 & 1.5 & 1.5 & 0.9 & 0.7 & 1.5 & 1.6 & \\
\hline & orf19.3021 & 0.8 & 1.5 & 1.9 & 2.0 & 1.4 & 1.0 & 1.5 & 1.7 & \\
\hline & orf19.5943 & 1.3 & 1.2 & 1.6 & 1.6 & 1.2 & 1.0 & 1.5 & 1.5 & \\
\hline
\end{tabular}


Appendix B. Continued.

\begin{tabular}{|c|c|c|c|c|c|c|c|c|c|c|}
\hline \multirow[b]{3}{*}{ CGD Name } & \multirow[b]{3}{*}{ orf19 Designation } & \multicolumn{8}{|c|}{ Change in fold expression ${ }^{a}$} & \multirow[b]{3}{*}{ Molecular function } \\
\hline & & \multicolumn{2}{|c|}{ G648S } & \multicolumn{2}{|c|}{$\underline{\text { G648D }}$} & \multicolumn{2}{|c|}{$\underline{\mathrm{Y} 642 \mathrm{~F}}$} & \multicolumn{2}{|c|}{$\underline{\mathrm{A} 643 \mathrm{~V}}$} & \\
\hline & & Exp1 & Exp2 & Exp1 & Exp2 & Exp1 & Exp2 & Exp1 & Exp2 & \\
\hline & orf19.7038 & 1.1 & 1.4 & 1.8 & 1.8 & 1.1 & 1.0 & 1.5 & 1.5 & phosphatidylinositol 3-phosphate binding (IEA) \\
\hline & orf19.6443 & 1.2 & 1.9 & 2.1 & 1.9 & 1.2 & 1.2 & 1.5 & 1.7 & dodecenoyl-CoA delta-isomerase activity (IEA) \\
\hline & orf19.3233 & 1.2 & 1.2 & 1.9 & 1.5 & 1.2 & 1.2 & 1.5 & 1.6 & protein binding (IEA) \\
\hline & orf19.6995 & 1.0 & 1.1 & 1.9 & 1.6 & 0.6 & 0.9 & 1.5 & 1.6 & \\
\hline & orf19.5236 & 1.2 & 1.4 & 1.5 & 1.5 & 1.3 & 1.0 & 1.5 & 1.7 & dehydrodolichyl diphosphate synthase activity (IEA) \\
\hline & orf19.6469 & 1.1 & 1.5 & 1.7 & 1.7 & 1.1 & 1.2 & 1.5 & 1.7 & * RNA-directed DNA polymerase activity \\
\hline & orf19.6440 & 1.1 & 1.2 & 1.7 & 1.5 & 1.0 & 0.9 & 1.5 & 1.5 & ubiquitin-protein ligase activity (IEA) \\
\hline & orf19.278 & 1.4 & 1.6 & 1.6 & 1.9 & 1.4 & 1.2 & 1.5 & 1.7 & \\
\hline & orf19.827 & 1.4 & 1.9 & 1.9 & 2.9 & 1.3 & 1.4 & 1.5 & 1.8 & \\
\hline & orf19.4070 & 1.2 & 1.6 & 1.7 & 1.6 & 1.3 & 1.2 & 1.5 & 1.7 & \\
\hline & orf19.5239 & 1.0 & 1.8 & 2.4 & 2.1 & 1.8 & 1.0 & 1.5 & 1.5 & \\
\hline & orf19.6048 & 0.8 & 1.3 & 1.5 & 1.5 & 0.8 & 1.0 & 1.5 & 1.6 & dolichyl-phosphate-mannose-protein mannosyltransferase activity (IEA) \\
\hline$A L S 1$ & orf19.5741 & 2.6 & 0.9 & 1.3 & 2.2 & 2.8 & 2.3 & 3.4 & 2.1 & protein binding (IDA)|cell adhesion molecule binding (IEA) \\
\hline IFM3 & orf19.2176 & 1.6 & 1.4 & 1.4 & 1.4 & 1.6 & 1.7 & 1.8 & 1.8 & \\
\hline \multirow[t]{4}{*}{ MSO1 } & orf19.5248 & 1.3 & 1.4 & 2.7 & 3.0 & 1.6 & 1.5 & 2.9 & 2.2 & \\
\hline & orf19.4041 & 1.8 & 1.3 & 1.2 & 2.4 & 1.9 & 1.5 & 3.2 & 2.3 & ubiquitin-protein ligase activity (IEA) \\
\hline & orf19.1050 & 1.3 & 2.5 & 0.8 & 3.2 & 1.9 & 2.9 & 1.6 & 1.9 & \\
\hline & orf19.2016 & 1.1 & 1.5 & 1.3 & 2.1 & 1.5 & 1.7 & 1.5 & 1.6 & protein binding, bridging (IEA) \\
\hline COQ4 & orf19.3008 & 1.7 & 1.4 & 2.6 & 2.8 & 1.8 & 1.5 & 2.9 & 2.3 & \\
\hline$D A L 1$ & orf19.5454 & 2.1 & 1.3 & 1.8 & 2.3 & 2.8 & 2.4 & 2.8 & 1.9 & \\
\hline$D A P 1$ & orf19.489 & 1.3 & 2.0 & 3.0 & 3.9 & 1.8 & 2.0 & 2.7 & 2.8 & heme binding (IEA) \\
\hline ERG11 & orf19.922 & 1.6 & 1.4 & 1.8 & 1.6 & 1.7 & 1.6 & 1.7 & 1.7 & drug binding (IDA, ISS)|sterol 14-demethylase activity (IDA) \\
\hline ERG6 & orf19.1631 & 1.6 & 1.4 & 2.1 & 1.5 & 1.8 & 1.8 & 1.7 & 1.9 & sterol 24-C-methyltransferase activity (IGI, IMP, ISS) \\
\hline$E R G 7$ & orf19.1570 & 1.4 & 1.6 & 2.4 & 2.1 & 1.6 & 1.7 & 2.4 & 2.7 & lanosterol synthase activity (IGI, ISS) \\
\hline
\end{tabular}


Appendix B. Continued.

\begin{tabular}{|c|c|c|c|c|c|c|c|c|c|c|}
\hline \multirow[b]{3}{*}{ CGD Name } & \multirow[b]{3}{*}{ orf19 Designation } & \multicolumn{8}{|c|}{ Change in fold expression ${ }^{\mathrm{a}}$} & \multirow[b]{3}{*}{ Molecular function } \\
\hline & & \multicolumn{2}{|c|}{$\underline{\text { G648S }}$} & \multicolumn{2}{|c|}{$\underline{\text { G648D }}$} & \multicolumn{2}{|c|}{$\underline{\mathrm{Y} 642 \mathrm{~F}}$} & \multicolumn{2}{|c|}{$\underline{\mathrm{A} 643 \mathrm{~V}}$} & \\
\hline & & Exp1 & Exp2 & Exp1 & Exp2 & Exp1 & Exp2 & Exp1 & Exp2 & \\
\hline$E R G 9$ & orf19.3616 & 1.5 & 1.3 & 2.0 & 1.7 & 1.7 & 1.5 & 1.8 & 1.8 & farnesyl-diphosphate farnesyltransferase activity (ISS) \\
\hline$E X G 2$ & orf19.2952 & 2.0 & 1.4 & 2.4 & 2.4 & 2.0 & 1.5 & 2.4 & 1.9 & glucan 1,3-beta-glucosidase activity (IDA, IGI, IMP) \\
\hline$I P K 2$ & orf19.1377 & 2.1 & 1.2 & 3.0 & 2.2 & 2.5 & 1.5 & 3.7 & 2.1 & inositol 1,3,4,5,6-pentakisphosphate kinase activity (IEA) \\
\hline PGA10 & orf19.5674 & 1.4 & 2.3 & 1.8 & 2.4 & 1.6 & 2.2 & 1.6 & 2.5 & heme binding (IDA, IGI) \\
\hline$S L D 1$ & orf19.260 & 1.6 & 1.4 & 1.9 & 2.0 & 1.7 & 1.6 & 1.9 & 1.8 & \\
\hline \multirow[t]{17}{*}{ SNO1 } & orf19.2948 & 1.4 & 1.4 & 2.4 & 2.0 & 1.8 & 1.5 & 2.3 & 1.9 & imidazoleglycerol-phosphate synthase activity (IEA) \\
\hline & orf19.692 & 1.3 & 2.7 & 2.2 & 3.4 & 1.7 & 1.8 & 3.0 & 4.1 & \\
\hline & orf19.1344 & 4.7 & 1.3 & 2.9 & 1.8 & 3.2 & 2.5 & 3.5 & 2.1 & \\
\hline & orf19.7504 & 2.2 & 1.4 & 4.0 & 2.4 & 2.4 & 1.8 & 2.6 & 1.8 & \\
\hline & orf19.2175 & 1.5 & 1.3 & 1.5 & 1.6 & 1.8 & 1.6 & 1.8 & 1.7 & \\
\hline & orf19.2452 & 2.2 & 1.3 & 2.1 & 1.9 & 2.2 & 1.5 & 2.7 & 1.7 & \\
\hline & orf19.4167 & 1.9 & 0.7 & 2.2 & 2.2 & 2.8 & 1.7 & 2.9 & 1.7 & \\
\hline & orf19.4690 & 1.2 & 5.0 & 11.3 & 10.3 & 1.9 & 2.4 & 3.4 & 6.7 & manganese ion transmembrane transporter activity (IEA) \\
\hline & orf19.3921 & 1.6 & 1.2 & 2.8 & 2.2 & 1.6 & 1.5 & 2.0 & 1.7 & \\
\hline & orf19.633 & 1.7 & 1.0 & 2.3 & 2.3 & 1.9 & 1.6 & 2.8 & 1.9 & \\
\hline & orf19.4459 & 1.6 & 1.2 & 3.5 & 3.5 & 2.1 & 1.7 & 2.4 & 2.0 & \\
\hline & orf19.6753 & 1.4 & 1.5 & 3.3 & 1.5 & 1.8 & 1.5 & 2.9 & 2.0 & \\
\hline & orf19.6558 & 1.7 & 1.4 & 1.7 & 1.7 & 1.7 & 1.5 & 1.8 & 1.5 & GTPase activator activity (IEA) \\
\hline & orf19.633 & 1.7 & 1.0 & 2.3 & 2.3 & 1.9 & 1.6 & 2.8 & 1.9 & \\
\hline & orf19.6558 & 1.7 & 1.4 & 1.7 & 1.7 & 1.7 & 1.5 & 1.8 & 1.5 & GTPase activator activity (IEA) \\
\hline & orf19.2296 & 2.2 & 2.4 & 3.6 & 1.2 & 1.7 & 1.9 & 2.7 & 1.9 & \\
\hline & orf19.6342 & 1.9 & 3.9 & 3.3 & 9.3 & 0.6 & 4.7 & 2.5 & 9.9 & \\
\hline AROS & orf19.1237 & 1.5 & 3.4 & 5.8 & 4.2 & 1.5 & 1.9 & 2.1 & 2.8 & aromatic-amino-acid:2-oxoglutarate aminotransferase activity (ISS) \\
\hline$C A G 1$ & orf19.4015 & 2.4 & 1.6 & 2.7 & 1.5 & 1.4 & 0.9 & 2.1 & 1.5 & GTPase activity (IGI)|guanyl nucleotide binding (ISS) \\
\hline
\end{tabular}


Appendix B. Continued.

\begin{tabular}{|c|c|c|c|c|c|c|c|c|c|c|}
\hline \multirow[b]{3}{*}{ CGD Name } & \multirow[b]{3}{*}{ orf19 Designation } & \multicolumn{8}{|c|}{ Change in fold expression ${ }^{a}$} & \multirow[b]{3}{*}{ Molecular function } \\
\hline & & \multicolumn{2}{|c|}{$\underline{\text { G648S }}$} & \multicolumn{2}{|c|}{$\underline{\text { G648D }}$} & \multicolumn{2}{|c|}{$\underline{\mathrm{Y} 642 \mathrm{~F}}$} & \multicolumn{2}{|c|}{ A643V } & \\
\hline & & Exp1 & Exp2 & Exp1 & Exp2 & Exp1 & Exp2 & Exp1 & Exp2 & \\
\hline CAN1 & orf19.97 & 1.6 & 1.7 & 5.5 & 3.2 & 1.2 & 1.1 & 2.5 & 2.4 & amino acid binding \\
\hline CDR11 & orf19.919 & 1.8 & 2.1 & 4.1 & 4.9 & 1.4 & 1.5 & 2.3 & 4.2 & ATP binding (ISS) \\
\hline $\mathrm{CNHI}$ & orf19.367 & 2.3 & 1.9 & 2.8 & 2.3 & 1.9 & 1.3 & 2.4 & 1.8 & Candida albicans $\mathrm{Na}+/ \mathrm{H}+$ antiporter \\
\hline ENT3 & orf19.1553 & 2.1 & 1.9 & 2.2 & 2.1 & 2.0 & 1.4 & 1.5 & 1.9 & \\
\hline HAP31 & orf19.517 & 1.5 & 1.7 & 2.3 & 2.4 & 1.8 & 1.4 & 2.0 & 1.9 & transcription factor activity (NAS)|transcription activator activity (IEA) \\
\hline HAP42 & orf19.1481 & 1.6 & 1.5 & 3.6 & 2.3 & 2.2 & 1.0 & 2.1 & 1.5 & \\
\hline IFRI & orf19.1763 & 1.6 & 3.3 & 6.8 & 2.9 & 1.9 & 1.1 & 2.6 & 2.4 & \\
\hline LIG4 & orf19.5798 & 1.7 & 1.5 & 3.3 & 2.4 & 1.9 & 1.2 & 2.6 & 1.9 & DNA ligase activity (IGI, ISS)|DNA ligase (ATP) activity (IEA) \\
\hline$P X P 2$ & orf19.1655 & 1.5 & 2.5 & 3.5 & 3.3 & 0.8 & 1.3 & 2.0 & 2.2 & acyl-CoA oxidase activity (ISS) \\
\hline UGA6 & orf19.5820 & 1.7 & 1.9 & 3.7 & 1.5 & 1.7 & 1.0 & 3.0 & 1.8 & amino acid transmembrane transporter activity \\
\hline \multirow[t]{14}{*}{ ZCF11 } & orf19.2423 & 1.7 & 1.5 & 2.8 & 2.5 & 2.1 & 1.4 & 2.9 & 2.3 & sequence-specific DNA binding (IEA) \\
\hline & orf19.4191.1 & 1.5 & 1.5 & 3.2 & 2.9 & 1.9 & 1.4 & 3.5 & 3.1 & ubiquitin-ubiquitin ligase activity (IEA) \\
\hline & orf19.2836 & 2.5 & 1.7 & 5.3 & 3.4 & 2.0 & 1.3 & 5.7 & 3.0 & \\
\hline & orf19.6970 & 1.8 & 1.9 & 3.8 & 3.8 & 2.6 & 1.4 & 4.7 & 2.8 & \\
\hline & orf19.3848 & 2.4 & 1.8 & 6.3 & 3.5 & 2.0 & 1.1 & 6.4 & 2.8 & \\
\hline & orf19.5799 & 1.7 & 2.0 & 3.4 & 2.4 & 1.5 & 1.5 & 2.4 & 2.6 & \\
\hline & orf19.6986 & 2.4 & 1.8 & 2.5 & 2.2 & 2.8 & 1.2 & 2.6 & 2.2 & \\
\hline & orf19.1881 & 1.5 & 1.6 & 2.4 & 2.2 & 1.5 & 1.5 & 2.4 & 2.1 & 1-acylglycerol-3-phosphate O-acyltransferase activity (IEA) \\
\hline & orf19.4763 & 1.6 & 2.0 & 2.7 & 3.1 & 1.7 & 1.1 & 2.3 & 2.0 & \\
\hline & orf19.1964 & 1.5 & 2.0 & 1.6 & 2.1 & 1.2 & 1.9 & 1.6 & 2.0 & \\
\hline & orf19.5644 & 1.9 & 1.6 & 3.1 & 2.2 & 1.7 & 1.2 & 2.6 & 1.9 & \\
\hline & orf19.4982 & 1.7 & 1.6 & 3.0 & 2.0 & 1.9 & 1.1 & 2.7 & 1.8 & \\
\hline & orf19.5037 & 3.1 & 1.5 & 2.3 & 2.0 & 2.0 & 1.2 & 2.7 & 1.8 & \\
\hline & orf19.541 & 1.6 & 1.6 & 1.7 & 1.9 & 1.6 & 1.2 & 2.2 & 1.7 & \\
\hline
\end{tabular}




\begin{tabular}{|c|c|c|c|c|c|c|c|c|c|c|}
\hline \multirow[b]{3}{*}{ CGD Name } & \multirow[b]{3}{*}{ orf19 Designation } & \multicolumn{8}{|c|}{ Change in fold expression ${ }^{\mathrm{a}}$} & \multirow[b]{3}{*}{ Molecular function } \\
\hline & & \multicolumn{2}{|c|}{$\underline{\text { G648S }}$} & \multicolumn{2}{|c|}{$\underline{\text { G648D }}$} & \multicolumn{2}{|c|}{$\underline{\mathrm{Y} 642 \mathrm{~F}}$} & \multicolumn{2}{|c|}{$\underline{\mathrm{A} 643 \mathrm{~V}}$} & \\
\hline & & Exp1 & Exp2 & Exp1 & Exp2 & Exp1 & Exp2 & Exp1 & Exp2 & \\
\hline & orf19.4170 & 2.3 & 1.7 & 2.8 & 2.5 & 2.9 & 1.3 & 4.2 & 1.7 & structural constituent of cell wall (IEA) \\
\hline & orf19.5426 & 2.0 & 1.9 & 3.0 & 1.8 & 1.6 & 1.2 & 2.1 & 1.7 & triglyceride lipase activity (IEA) \\
\hline & orf19.7380 & 1.5 & 2.1 & 2.4 & 2.3 & 1.3 & 1.2 & 1.6 & 1.6 & \\
\hline & orf19.6797 & 1.5 & 2.1 & 1.5 & 1.8 & 1.1 & 1.7 & 1.8 & 1.8 & \\
\hline & orf19.4657 & 2.1 & 1.5 & 2.8 & 2.0 & 2.1 & 1.4 & 2.5 & 1.6 & contributes_to phosphoprotein phosphatase activity (IEA) \\
\hline & orf19.1504 & 1.6 & 1.6 & 2.7 & 1.6 & 1.8 & 1.0 & 3.0 & 1.6 & \\
\hline & orf19.7345 & 1.7 & 1.6 & 2.1 & 2.0 & 1.8 & 1.1 & 2.6 & 1.6 & ATP binding (IEA) \\
\hline & orf19.4346 & 1.6 & 1.6 & 2.4 & 1.8 & 1.6 & 0.9 & 2.1 & 1.5 & protein anchor \\
\hline & orf19.4589 & 1.5 & 1.5 & 1.8 & 1.6 & 1.3 & 1.3 & 1.7 & 1.5 & amine oxidase activity (IEA) \\
\hline & orf19.2607 & 1.7 & 1.5 & 2.2 & 1.7 & 2.0 & 1.3 & 1.8 & 1.5 & \\
\hline & orf19.6348 & 1.7 & 2.0 & 2.0 & 2.2 & 1.5 & 1.2 & 2.4 & 1.5 & \\
\hline \multirow[t]{3}{*}{ PRM9 } & orf19.2508 & 14.1 & 2.9 & 15.1 & 1.6 & 13.3 & 2.6 & 13.7 & 1.2 & \\
\hline & orf19.1444 & 2.0 & 1.7 & 4.4 & 1.9 & 2.9 & 1.6 & 2.6 & 1.4 & phosphatidylinositol-4,5-bisphosphate binding \\
\hline & orf19.4330 & 1.7 & 2.0 & 2.3 & 1.9 & 1.7 & 1.5 & 1.4 & 1.8 & \\
\hline ARO9 & orf19.1237 & 1.5 & 3.4 & 5.8 & 4.2 & 1.5 & 1.9 & 2.1 & 2.8 & aromatic-amino-acid:2-oxoglutarate aminotransferase activity (ISS) \\
\hline BTS1 & orf19.6674 & 2.0 & 2.5 & 6.8 & 6.4 & 2.6 & 2.0 & 5.0 & 4.1 & farnesyltranstransferase activity (IEA) \\
\hline$B U L 1$ & orf19.5094 & 1.9 & 1.9 & 4.1 & 3.1 & 2.5 & 1.8 & 2.1 & 1.9 & \\
\hline COQ4 & orf19.3008 & 1.5 & 1.5 & 2.3 & 2.8 & 1.9 & 1.6 & 2.6 & 2.5 & \\
\hline DDR48 & orf19.4082 & 2.2 & 4.3 & 5.8 & 4.7 & 2.7 & 2.9 & 3.4 & 4.3 & \\
\hline$E R G 1$ & orf19.406 & 1.8 & 1.8 & 2.7 & 2.3 & 2.2 & 2.0 & 2.3 & 2.3 & squalene monooxygenase activity (IDA, IMP)|drug binding (IEA) \\
\hline$E R G 2$ & orf19.6026 & 1.7 & 1.5 & 2.3 & 1.9 & 1.9 & 1.9 & 2.1 & 2.0 & C-8 sterol isomerase activity (IGI, IMP) \\
\hline$E R G 24$ & orf19.1598 & 1.7 & 1.7 & 2.3 & 2.1 & 1.7 & 1.8 & 2.1 & 2.3 & delta14-sterol reductase activity (IGI, ISS) \\
\hline$E R G 26$ & orf19.2909 & 1.7 & 1.6 & 2.7 & 2.6 & 2.2 & 2.0 & 2.7 & 2.7 & C-3 sterol dehydrogenase (C-4 sterol decarboxylase) activity (IGI, ISS) \\
\hline$E R G 27$ & orf19.3240 & 1.8 & 2.1 & 3.3 & 3.4 & 2.2 & 2.2 & 3.1 & 3.2 & 3-keto sterol reductase activity (IGI, IMP) \\
\hline
\end{tabular}


Appendix B. Continued.

\begin{tabular}{|c|c|c|c|c|c|c|c|c|c|c|}
\hline \multirow[b]{3}{*}{ CGD Name } & \multirow[b]{3}{*}{ orf19 Designation } & \multicolumn{8}{|c|}{ Change in fold expression ${ }^{\mathrm{a}}$} & \multirow[b]{3}{*}{ Molecular function } \\
\hline & & \multicolumn{2}{|c|}{$\underline{\text { G648S }}$} & \multicolumn{2}{|c|}{$\underline{\text { G648D }}$} & \multicolumn{2}{|c|}{$\underline{\mathrm{Y} 642 \mathrm{~F}}$} & \multicolumn{2}{|c|}{$\underline{\mathrm{A} 643 \mathrm{~V}}$} & \\
\hline & & Exp1 & Exp2 & Exp1 & Exp2 & Exp1 & Exp2 & Exp1 & Exp2 & \\
\hline$E R G 7$ & orf19.1570 & 1.5 & 1.6 & 2.8 & 2.1 & 1.8 & 1.6 & 2.7 & 2.4 & lanosterol synthase activity (IGI, ISS) \\
\hline FMA1 & orf19.6837 & 1.8 & 3.2 & 2.7 & 5.3 & 1.5 & 3.3 & 1.7 & 3.0 & nucleotide binding, oxidoreductase activity \\
\hline FRP1 & orf19.5634 & 5.4 & 5.7 & 17.3 & 17.5 & 8.1 & 5.9 & 16.3 & 14.7 & ferric-chelate reductase activity (IEA, IMP) \\
\hline FTH1 & orf19.4802 & 2.6 & 2.6 & 5.3 & 5.2 & 2.3 & 1.9 & 6.6 & 5.2 & \\
\hline GYP1 & orf19.3811 & 1.6 & 1.6 & 3.7 & 3.5 & 1.8 & 1.5 & 3.7 & 3.4 & Rab GTPase activator activity (IEA) \\
\hline HEM14 & orf19.4747 & 2.1 & 1.6 & 3.6 & 3.0 & 2.2 & 1.8 & 2.9 & 2.3 & oxygen-dependent protoporphyrinogen oxidase activity (ISS) \\
\hline HXT5 & orf19.4384 & 1.8 & 2.1 & 2.6 & 2.0 & 1.8 & 1.5 & 2.3 & 1.7 & hexose transmembrane transporter activity (IEP) \\
\hline$I D P 2$ & orf19.3733 & 2.2 & 1.6 & 3.3 & 2.8 & 2.6 & 2.0 & 3.2 & 2.5 & isocitrate dehydrogenase (NADP+) activity (IEA) \\
\hline IFD7 & orf19.629 & 1.7 & 3.2 & 4.6 & 6.8 & 1.7 & 2.7 & 1.7 & 4.3 & \\
\hline$M D R 1$ & orf19.5604 & 5.9 & 5.5 & 15.1 & 8.0 & 6.6 & 6.1 & 5.9 & 5.7 & drug transporter activity (ISS)|fluconazole transporter activity (IDA) \\
\hline$N C P 1$ & orf19.2672 & 1.6 & 1.7 & 2.6 & 2.0 & 1.7 & 1.9 & 1.9 & 1.9 & NADPH-hemoprotein reductase activity (ISS)|electron carrier activity (IEA) \\
\hline PGA45 & orf19.2451 & 1.7 & 1.5 & 2.1 & 2.2 & 1.7 & 1.9 & 2.0 & 2.3 & \\
\hline$P G A 7$ & orf19.5635 & 2.3 & 3.7 & 5.7 & 7.4 & 2.7 & 3.8 & 4.8 & 6.1 & \\
\hline PHOI00 & orf19.4424 & 1.5 & 3.4 & 4.8 & 2.7 & 1.6 & 1.5 & 2.0 & 2.3 & acid phosphatase activity (NAS) \\
\hline SET3 & orf19.7221 & 2.8 & 2.7 & 6.3 & 5.7 & 4.4 & 3.6 & 4.8 & 4.8 & NAD-dependent histone deacetylase activity (IEA) \\
\hline$S L D 1$ & orf19.260 & 1.8 & 1.5 & 2.2 & 1.8 & 1.7 & 1.6 & 2.0 & 1.9 & oxidoreductase activity \\
\hline TEF4 & orf19.2652 & 16.4 & 16.6 & 55.8 & 90.2 & 29.9 & 32.7 & 65.5 & 67.8 & translation elongation factor activity (IEA) \\
\hline \multirow[t]{7}{*}{$Y M X 6$} & orf19.5713 & 1.6 & 2.6 & 2.9 & 2.0 & 1.6 & 1.6 & 1.7 & 1.8 & NADH dehydrogenase activity (IEA) \\
\hline & orf19.1801 & 1.7 & 1.6 & 2.4 & 2.0 & 1.9 & 1.6 & 2.0 & 1.8 & cytochrome-b5 reductase activity (IEA) \\
\hline & orf19.5535 & 1.5 & 1.6 & 2.2 & 2.7 & 1.5 & 1.9 & 2.7 & 3.2 & anion:cation symporter activity (ISS)|pantothenate transporter activity (IEA) \\
\hline & orf19.6169.2 & 1.7 & 1.8 & 2.6 & 2.5 & 1.8 & 1.7 & 2.0 & 1.5 & \\
\hline & orf19.288 & 1.8 & 1.5 & 3.0 & 2.5 & 2.1 & 1.5 & 2.5 & 2.0 & methylenetetrahydrofolate reductase (NADPH) activity (IEA) \\
\hline & orf19.329 & 2.1 & 2.3 & 2.4 & 2.6 & 2.0 & 1.6 & 2.5 & 2.5 & RNA binding (IEA) \\
\hline & orf19.7547 & 1.8 & 1.5 & 2.8 & 2.7 & 2.0 & 1.5 & 3.1 & 2.3 & phosphatidylinositol 3-phosphate binding (IEA)|ubiquitin-protein ligase activity (IEA) \\
\hline
\end{tabular}


Appendix B. Continued.

\begin{tabular}{|c|c|c|c|c|c|c|c|c|c|c|}
\hline \multirow[b]{3}{*}{ CGD Name } & \multirow[b]{3}{*}{ orf19 Designation } & \multicolumn{8}{|c|}{ Change in fold expression ${ }^{\mathrm{a}}$} & \multirow[b]{3}{*}{ Molecular function } \\
\hline & & \multicolumn{2}{|c|}{$\underline{\text { G648S }}$} & \multicolumn{2}{|c|}{$\underline{\text { G648D }}$} & \multicolumn{2}{|c|}{$\underline{Y 642 F}$} & \multicolumn{2}{|c|}{$\underline{\mathrm{A} 643 \mathrm{~V}}$} & \\
\hline & & Exp1 & Exp2 & Exp1 & Exp2 & Exp1 & Exp2 & Exp1 & Exp2 & \\
\hline & orf19.7329 & 1.6 & 2.1 & 3.1 & 3.0 & 1.7 & 1.8 & 2.5 & 2.3 & ubiquitin-protein ligase activity (IEA) \\
\hline & orf19.3627 & 4.0 & 1.9 & 7.0 & 4.0 & 4.4 & 1.9 & 5.1 & 1.9 & \\
\hline & orf19.5210 & 1.5 & 2.1 & 2.7 & 2.7 & 1.8 & 1.5 & 2.9 & 2.3 & transcription factor activity (IEA) \\
\hline & orf19.2350 & 1.7 & 1.7 & 3.4 & 4.4 & 2.0 & 1.9 & 3.2 & 3.0 & drug transporter activity (ISS) \\
\hline & orf19.3483 & 2.0 & 2.4 & 5.3 & 4.7 & 2.9 & 2.2 & 3.5 & 3.3 & phosphatidylglycerol phospholipase C activity (IEA) \\
\hline & orf19.344 & 8.2 & 9.0 & 25.0 & 20.7 & 9.7 & 7.3 & 13.3 & 10.2 & \\
\hline & orf19.5777 & 1.7 & 2.4 & 4.8 & 3.8 & 1.9 & 1.5 & 3.1 & 2.5 & \\
\hline & orf19.5799 & 1.7 & 2.0 & 3.4 & 2.4 & 1.5 & 1.5 & 2.4 & 2.6 & \\
\hline & orf19.3261 & 1.8 & 2.2 & 2.7 & 3.4 & 1.6 & 2.3 & 4.5 & 4.5 & \\
\hline & orf19.7456 & 1.7 & 2.6 & 4.4 & 4.1 & 2.2 & 2.1 & 3.9 & 3.5 & \\
\hline & orf19.7043 & 2.5 & 1.9 & 5.2 & 3.6 & 2.8 & 2.3 & 4.9 & 3.0 & \\
\hline & orf19.4013 & 2.6 & 2.2 & 4.2 & 3.5 & 3.1 & 2.8 & 4.8 & 3.8 & \\
\hline & orf19.4014 & 1.5 & 2.1 & 2.3 & 2.4 & 1.7 & 1.6 & 2.6 & 2.6 & \\
\hline & orf19.4031 & 1.8 & 1.8 & 2.5 & 2.5 & 1.6 & 1.5 & 2.9 & 2.5 & \\
\hline & orf19.6840 & 2.0 & 2.1 & 4.1 & 4.4 & 2.6 & 2.4 & 3.1 & 2.6 & \\
\hline & orf19.286 & 2.1 & 2.5 & 2.3 & 2.2 & 1.9 & 1.9 & 3.5 & 1.9 & \\
\hline & orf19.1865 & 2.4 & 2.2 & 3.7 & 2.9 & 2.4 & 2.2 & 3.4 & 3.1 & \\
\hline & orf19.7504 & 2.1 & 1.8 & 3.0 & 3.3 & 1.9 & 2.0 & 2.2 & 2.1 & \\
\hline & orf19.3737 & 1.6 & 1.9 & 2.9 & 3.4 & 2.0 & 1.8 & 3.1 & 2.6 & \\
\hline & orf19.7263 & 1.8 & 1.9 & 2.9 & 2.8 & 1.6 & 1.7 & 2.1 & 1.9 & \\
\hline & orf19.1964 & 2.0 & 1.8 & 2.5 & 2.2 & 1.7 & 1.6 & 2.2 & 1.6 & \\
\hline & orf19.1800 & 2.3 & 2.2 & 4.6 & 3.9 & 2.5 & 2.2 & 4.5 & 3.4 & \\
\hline & orf19.6025 & 2.2 & 2.1 & 3.9 & 5.2 & 3.8 & 3.1 & 5.2 & 5.2 & $\mathrm{~N}$-acetylglucosaminyldiphosphodolichol $\mathrm{N}$-acetylglucosaminyltransferase activity \\
\hline & orf19.2691 & 1.5 & 5.4 & 5.4 & 9.1 & 1.6 & 2.3 & 4.4 & 6.5 & \\
\hline
\end{tabular}


Appendix B. Continued.

\begin{tabular}{|c|c|c|c|c|c|c|c|c|c|c|}
\hline \multirow[b]{3}{*}{ CGD Name } & \multirow[b]{3}{*}{ orf19 Designation } & \multicolumn{8}{|c|}{ Change in fold expression ${ }^{a}$} & \multirow[b]{3}{*}{ Molecular function } \\
\hline & & \multicolumn{2}{|c|}{$\underline{\text { G648S }}$} & \multicolumn{2}{|c|}{$\underline{\text { G648D }}$} & \multicolumn{2}{|c|}{$\underline{Y 642 F}$} & \multicolumn{2}{|c|}{$\underline{\mathrm{A} 643 \mathrm{~V}}$} & \\
\hline & & Exp1 & Exp2 & Exp1 & Exp2 & Exp1 & Exp2 & Exp1 & Exp2 & \\
\hline & orf19.496 & 2.4 & 2.3 & 4.4 & 3.8 & 2.5 & 2.0 & 3.6 & 3.1 & DNA-dependent ATPase activity (IEA) \\
\hline & orf19.1881 & 1.5 & 1.6 & 2.4 & 2.2 & 1.5 & 1.5 & 2.4 & 2.1 & 1-acylglycerol-3-phosphate O-acyltransferase activity (IEA) \\
\hline & orf19.2285 & 1.7 & 2.5 & 1.8 & 1.5 & 1.8 & 2.4 & 2.1 & 2.1 & \\
\hline
\end{tabular}

${ }^{a}$ Fold change defined as the average ratio of gene expression levels in the isolates compared in two independent microarray experiments.

Bold indicates "real" result as defined in Materials and Methods

Red shading indicates ratio values above 1.5 . 
APPENDIX C. GENES DOWN-REGULATED IN STRAINS CARRYING UPC2 GOF ALLELE BY AT LEAST 1.5 FOLD

\begin{tabular}{|c|c|c|c|c|c|c|c|c|c|c|}
\hline & & \multicolumn{8}{|c|}{ Change in fold expression ${ }^{\mathrm{a}}$} & \\
\hline & & \multicolumn{2}{|c|}{$\underline{\text { G648S }}$} & \multicolumn{2}{|c|}{$\underline{\text { G648D }}$} & \multicolumn{2}{|c|}{$\underline{\mathbf{Y 6 4 2 F}}$} & \multicolumn{2}{|c|}{$\underline{\mathbf{A} 643 \mathrm{~V}}$} & \\
\hline \multicolumn{11}{|c|}{ CGD Name orf19 Designation Exp1 Exp2 Exp1 Exp2 Exp1 Exp2 Exp1 Exp2 Molecular function } \\
\hline$A B P 140$ & orf19.3676 & 1.6 & 0.4 & 0.5 & 0.5 & 1.7 & 1.0 & 0.8 & 0.5 & \\
\hline$A D H 3$ & orf19.4505 & 0.5 & 1.8 & 0.6 & 0.6 & 0.7 & 1.4 & 0.8 & 0.8 & alcohol dehydrogenase (NAD) activity (IEA) \\
\hline$A G P 2$ & orf19.4679 & 1.1 & 0.8 & 0.6 & 0.6 & 0.8 & 0.7 & 0.9 & 0.6 & amino acid transmembrane transporter activity (IEA) \\
\hline$A R X 1$ & orf19.3015 & 1.2 & 0.5 & 0.4 & 0.6 & 1.2 & 1.0 & 0.7 & 0.6 & \\
\hline$A Y R 2$ & orf19.5615 & 0.7 & 1.1 & 0.6 & 0.6 & 0.6 & 0.7 & 0.7 & 0.9 & acylglycerone-phosphate reductase activity (IEA) \\
\hline BUD21 & orf19.5430 & 1.1 & 0.6 & 0.4 & 0.4 & 1.2 & 1.2 & 0.7 & 0.5 & snoRNA binding (IEA) \\
\hline BUD22 & orf19.3287 & 1.5 & 0.6 & 0.4 & 0.5 & 1.3 & 0.9 & 0.7 & 0.4 & \\
\hline CAN2 & orf19.111 & 0.8 & 0.8 & 0.6 & 0.6 & 0.6 & 0.7 & 0.7 & 0.7 & arginine transmembrane transporter activity \\
\hline$C C W 14$ & orf19.1969 & 0.8 & 0.6 & 0.4 & 0.6 & 0.8 & 0.5 & 0.9 & 0.5 & structural constituent of cell wall (IEA) \\
\hline$C D R 1$ & orf19.6000 & 1.0 & 0.7 & 0.5 & 0.6 & 0.9 & 0.8 & 0.8 & 0.8 & ATP binding (IDA, IGI, IMP, ISS) \\
\hline$C D R 3$ & orf19.1313 & 0.9 & 0.8 & 0.6 & 0.6 & 0.8 & 0.8 & 0.8 & 0.7 & \\
\hline CHRI & orf19.3756 & 2.0 & 0.5 & 0.6 & 0.5 & 2.3 & 0.8 & 0.8 & 0.3 & ATP-dependent RNA helicase activity (ISS) \\
\hline CHS6 & orf19.5155 & 0.9 & 0.7 & 0.6 & 0.4 & 0.7 & 0.5 & 0.8 & 0.4 & \\
\hline$C I C l$ & orf19.124 & 1.9 & 0.5 & 0.5 & 0.5 & 1.8 & 1.1 & 0.9 & 0.5 & protein binding, bridging (IEA) \\
\hline CNS1 & orf19.6052 & 1.2 & 0.7 & 0.6 & 0.6 & 1.3 & 1.0 & 0.7 & 0.6 & Hsp70 protein binding (IEA) $\mid \mathrm{Hsp} 90$ protein binding (IEA) \\
\hline CSI2 & orf19.5232 & 1.5 & 0.5 & 0.5 & 0.6 & 1.7 & 1.0 & 1.0 & 0.5 & 5S rRNA binding (IEA)|rRNA primary transcript binding (IEA) \\
\hline$D B P 7$ & orf19.6902 & 1.6 & 0.5 & 0.5 & 0.6 & 1.5 & 1.1 & 0.9 & 0.6 & ATP-dependent RNA helicase activity (IEA) \\
\hline$D B P 8$ & orf19.6652 & 1.6 & 0.5 & 0.5 & 0.6 & 1.8 & 0.9 & 0.7 & 0.3 & ATP-dependent RNA helicase activity (IEA) \\
\hline DFII & orf19.7084 & 0.9 & 0.8 & 0.5 & 0.6 & 0.8 & 1.0 & 0.7 & 0.8 & \\
\hline$D I P 2$ & orf19.5106 & 1.5 & 0.6 & 0.5 & 0.5 & 1.4 & 1.1 & 0.8 & 0.5 & snoRNA binding (IEA) \\
\hline$D L D 1$ & orf19.5805 & 0.8 & 1.0 & 0.6 & 0.6 & 0.6 & 0.8 & 0.5 & 0.7 & D-lactate dehydrogenase (cytochrome) activity (IEA) \\
\hline ECMI & orf19.5299 & 1.9 & 0.6 & 0.5 & 0.6 & 1.6 & 0.9 & 0.8 & 0.4 & \\
\hline
\end{tabular}


Appendix C. Continued

\begin{tabular}{|c|c|c|c|c|c|c|c|c|c|c|}
\hline & & \multicolumn{8}{|c|}{ Change in fold expression ${ }^{\mathrm{a}}$} & \\
\hline & & \multicolumn{2}{|c|}{ G648S } & \multicolumn{2}{|c|}{$\underline{\text { G648D }}$} & \multicolumn{2}{|c|}{$\underline{Y 642 F}$} & \multicolumn{2}{|c|}{$\underline{\mathrm{A} 643 \mathrm{~V}}$} & \\
\hline \multicolumn{11}{|c|}{ CGD Name orf19 Designation Exp1 Exp2 Exp1 Exp2 Exp1 Exp2 Exp1 Exp2 Molecular function } \\
\hline ENP1 & orf19.5507 & 1.5 & 0.6 & 0.5 & 0.4 & 1.3 & 1.1 & 0.7 & 0.4 & snoRNA binding (IEA) \\
\hline ENP2 & orf19.6686 & 1.5 & 0.5 & 0.3 & 0.5 & 1.6 & 1.0 & 0.9 & 0.3 & \\
\hline FAD3 & orf19.4933 & 1.1 & 0.5 & 0.3 & 0.5 & 1.0 & 1.0 & 0.8 & 0.6 & omega-3 fatty acid desaturase activity (IMP) \\
\hline FCR1 & orf19.6817 & 0.7 & 0.9 & 0.6 & 0.6 & 0.6 & 0.7 & 0.7 & 0.7 & purine nucleotide binding (ISS)|transcription factor activity (IGI, ISS) \\
\hline FCY21 & orf19.1357 & 1.2 & 1.0 & 0.6 & 0.6 & 1.0 & 0.4 & 1.0 & 0.3 & \\
\hline GAL102 & orf19.3674 & 0.7 & 0.5 & 0.6 & 0.4 & 1.1 & 0.4 & 0.8 & 0.5 & UDP-glucose 4-epimerase activity (IEA) \\
\hline GAP4 & orf19.4456 & 0.9 & 0.8 & 0.6 & 0.6 & 0.8 & 0.9 & 0.7 & 0.7 & \\
\hline GAT2 & orf19.4056 & 1.2 & 1.4 & 0.4 & 0.5 & 0.8 & 1.0 & 0.6 & 0.4 & transcription factor activity (IEA) \\
\hline$H B R 3$ & orf19.6955 & 1.3 & 0.6 & 0.5 & 0.5 & 1.5 & 1.3 & 0.7 & 0.5 & SSU rRNA binding (IEA)|endoribonuclease activity (IEA) \\
\hline HCA4 & orf19.2712 & 1.5 & 0.5 & 0.6 & 0.5 & 1.8 & 1.0 & 0.8 & 0.5 & \\
\hline$H G T 8$ & orf19.2021 & 0.8 & 0.8 & 0.5 & 0.5 & 0.7 & 0.7 & 0.6 & 0.7 & glucose transmembrane transporter activity (ISS) \\
\hline IFD6 & orf19.1048 & 0.7 & 0.7 & 0.6 & 0.4 & 0.9 & 0.6 & 0.8 & 0.6 & aryl-alcohol dehydrogenase activity (NAS) \\
\hline$I P T 1$ & orf19.4769 & 0.8 & 0.8 & 0.5 & 0.6 & 0.6 & 0.8 & 0.7 & 0.6 & transferase activity, transferring phosphorus-containing groups (IMP, ISS) \\
\hline KTI12 & orf19.2385 & 1.2 & 0.7 & 0.5 & 0.5 & 1.5 & 0.9 & 0.7 & 0.5 & chromatin binding (IEA)|enzyme regulator activity (IEA) \\
\hline$M D N 1$ & orf19.4697 & 1.4 & 1.1 & 0.6 & 0.3 & 1.1 & 1.1 & 0.8 & 0.5 & ATPase activity (IEA) \\
\hline MET10 & orf19.4076 & 0.9 & 0.7 & 0.5 & 0.6 & 0.7 & 0.8 & 0.7 & 0.7 & sulfite reductase (NADPH) activity (IEA)|contributes_to sulfite reductase (NADPH) activity (IEA) \\
\hline MET14 & orf19.946 & 0.9 & 0.8 & 0.6 & 0.5 & 0.9 & 1.0 & 1.0 & 0.9 & adenylylsulfate kinase activity (IEA) \\
\hline MET3 & orf19.5025 & 1.4 & 0.9 & 0.4 & 0.4 & 1.1 & 0.8 & 1.3 & 0.8 & sulfate adenylyltransferase (ATP) activity (ISS) \\
\hline$N G T 1$ & orf19.5392 & 1.6 & 0.4 & 0.5 & 0.6 & 1.0 & 0.8 & 1.1 & 0.7 & $\mathrm{~N}$-acetylglucosamine transmembrane transporter activity (IDA, IMP, ISS) \\
\hline NIP7 & orf19.3478 & 1.4 & 0.5 & 0.4 & 0.5 & 1.2 & 1.0 & 0.7 & 0.4 & protein binding (IEA) \\
\hline NOC4 & orf19.1902 & 1.4 & 0.5 & 0.4 & 0.4 & 1.4 & 1.0 & 0.7 & 0.5 & \\
\hline NSAI & orf19.2185 & 1.3 & 0.5 & 0.5 & 0.6 & 1.7 & 1.1 & 0.7 & 0.5 & \\
\hline$P G A 26$ & orf19.2475 & 0.6 & 0.8 & 0.5 & 0.5 & 0.5 & 0.7 & 0.6 & 0.7 & \\
\hline
\end{tabular}


Appendix C. Continued

\begin{tabular}{|c|c|c|c|c|c|c|c|c|c|c|}
\hline & & \multicolumn{8}{|c|}{ Change in fold expression ${ }^{\mathrm{a}}$} & \\
\hline & & \multicolumn{2}{|c|}{$\underline{\text { G648S }}$} & \multicolumn{2}{|c|}{$\underline{\text { G648D }}$} & \multicolumn{2}{|c|}{$\underline{\mathrm{Y} 642 \mathrm{~F}}$} & \multicolumn{2}{|c|}{$\underline{\mathrm{A} 643 \mathrm{~V}}$} & \\
\hline \multicolumn{11}{|c|}{ CGD Name orf19 Designation Exp1 Exp2 Exp1 Exp2 Exp1 Exp2 Exp1 Exp2 Molecular function } \\
\hline$P G A 32$ & orf19.6784 & 0.9 & 0.9 & 0.6 & 0.5 & 0.8 & 0.8 & 0.7 & 0.6 & \\
\hline PHO113 & orf19.2619 & 0.8 & 0.7 & 0.6 & 0.6 & 0.8 & 0.8 & 0.7 & 0.7 & acid phosphatase activity (IEA) \\
\hline POL93 & orf19.6078 & 1.2 & 0.7 & 0.6 & 0.6 & 1.0 & 0.8 & 0.9 & 0.6 & DNA binding, RNA binding, RNA directed DNA polymerase activity \\
\hline POL93 & orf19.6078 & 1.0 & 0.5 & 0.3 & 0.4 & 0.8 & 0.8 & 0.8 & 0.7 & \\
\hline POP3 & orf19.7657 & 1.2 & 0.7 & 0.5 & 0.5 & 1.3 & 1.1 & 0.7 & 0.5 & ribonuclease MRP activity (IEA) \\
\hline POP4 & orf19.5567 & 1.2 & 0.7 & 0.6 & 0.6 & 1.3 & 0.9 & 0.7 & 0.6 & ribonuclease MRP activity (IEA) \\
\hline PRN4 & orf19.2461 & 0.9 & 0.7 & 0.6 & 0.5 & 0.9 & 0.6 & 0.7 & 0.6 & \\
\hline PUT1 & orf19.4274 & 1.2 & 0.8 & 0.6 & 0.5 & 1.0 & 0.7 & 0.7 & 0.6 & proline dehydrogenase activity (IEA) \\
\hline PUT2 & orf19.3974 & 1.2 & 0.9 & 0.6 & 0.6 & 1.0 & 0.8 & 0.8 & 0.8 & 1-pyrroline-5-carboxylate dehydrogenase activity (IEA) \\
\hline$P W P 2$ & orf19.3276 & 1.4 & 0.5 & 0.5 & 0.5 & 1.4 & 1.0 & 0.7 & 0.5 & snoRNA binding (IEA) \\
\hline$R C L 1$ & orf19.1886 & 1.4 & 0.7 & 0.6 & 0.5 & 1.3 & 0.9 & 0.8 & 0.6 & RNA-3'-phosphate cyclase activity (IEA \\
\hline REII & orf19.59 & 1.8 & 0.5 & 0.5 & 0.5 & 1.7 & 1.0 & 0.8 & 0.4 & sequence-specific DNA binding (IEA) \\
\hline RHD3 & orf19.5305 & 0.8 & 0.9 & 0.4 & 0.5 & 0.7 & 0.8 & 0.6 & 0.8 & \\
\hline RIM2 & orf19.4499 & 1.1 & 0.7 & 0.6 & 0.6 & 0.9 & 1.0 & 0.7 & 0.6 & pyrimidine nucleotide transmembrane transporter activity (IEA) \\
\hline$R L M 1$ & orf19.4662 & 1.2 & 0.8 & 0.5 & 0.6 & 1.0 & 0.8 & 0.9 & 0.5 & transcription regulator activity (IMP)|DNA bending activity (IEA) \\
\hline RPA12 & orf19.2287 & 1.8 & 0.5 & 0.5 & 0.5 & 1.7 & 1.0 & 1.0 & 0.4 & DNA-directed RNA polymerase activity (IEA)|exoribonuclease activity (IEA) \\
\hline$R P F 1$ & orf19.2667 & 1.5 & 0.5 & 0.5 & 0.6 & 1.4 & 1.1 & 1.0 & 0.6 & rRNA primary transcript binding (IEA) \\
\hline RRN11 & orf19.718 & 1.3 & 0.5 & 0.6 & 0.6 & 1.4 & 0.9 & 0.9 & 0.4 & transcription factor activity (ISS)|RNA polymerase I transcription factor activity (IEA) \\
\hline$R R P 15$ & orf19.563 & 1.6 & 0.5 & 0.5 & 0.5 & 1.6 & 1.0 & 0.7 & 0.5 & \\
\hline RTA2 & orf19.24 & 0.9 & 1.0 & 0.6 & 0.5 & 0.7 & 0.7 & 0.8 & 0.7 & phospholipid-translocating ATPase activity (IEA) \\
\hline$S D A 1$ & orf19.6648 & 1.4 & 0.5 & 0.4 & 0.5 & 1.4 & 1.0 & 0.8 & 0.5 & \\
\hline SKN1 & orf19.7362 & 1.5 & 0.4 & 0.3 & 0.3 & 1.2 & 0.7 & 0.8 & 0.3 & \\
\hline$S N G 3$ & orf19.1333 & 1.0 & 0.8 & 0.6 & 0.6 & 0.8 & 0.5 & 0.8 & 0.6 & \\
\hline
\end{tabular}


Appendix C. Continued

\begin{tabular}{|c|c|c|c|c|c|c|c|c|c|c|}
\hline & & \multicolumn{8}{|c|}{ Change in fold expression ${ }^{a}$} & \\
\hline & & \multicolumn{2}{|c|}{$\underline{\text { G648S }}$} & \multicolumn{2}{|c|}{ G648D } & \multicolumn{2}{|c|}{$\underline{Y 642 F}$} & \multicolumn{2}{|c|}{$\underline{\mathrm{A} 643 \mathrm{~V}}$} & \\
\hline \multicolumn{11}{|c|}{ CGD Name orf19 Designation Exp1 Exp2 Exp1 Exp2 Exp1 Exp2 Exp1 Exp2 Molecular function } \\
\hline SPC98 & orf19.2600 & 0.9 & 0.9 & 0.4 & 0.6 & 1.2 & 0.7 & 0.8 & 0.6 & structural constituent of cytoskeleton (IEA) \\
\hline$S Y G 1$ & orf19.768 & 0.8 & 0.9 & 0.5 & 0.6 & 0.8 & 0.7 & 0.7 & 0.6 & \\
\hline THI4 & orf19.5986 & 0.9 & 1.0 & 0.6 & 0.5 & 0.7 & 0.9 & 0.6 & 0.7 & \\
\hline TRM2 & orf19.3327 & 1.4 & 0.7 & 0.5 & 0.5 & 1.5 & 1.0 & 0.7 & 0.4 & double-stranded DNA specific 5'-3' exodeoxyribonuclease activity (IEA) \\
\hline TSR2 & orf19.2998 & 1.4 & 0.5 & 0.5 & 0.6 & 1.6 & 1.0 & 0.8 & 0.5 & \\
\hline$U L P 1$ & orf19.353 & 0.8 & 0.6 & 0.6 & 0.6 & 1.0 & 0.8 & 0.8 & 0.6 & SUMO-specific protease activity (IDA) \\
\hline UTP15 & orf19.3609 & 1.1 & 0.6 & 0.6 & 0.6 & 1.3 & 0.9 & 0.7 & 0.6 & snoRNA binding (IEA) \\
\hline UTP21 & orf19.1566 & 1.6 & 0.5 & 0.6 & 0.5 & 1.4 & 0.8 & 0.7 & 0.4 & \\
\hline UTP4 & orf19.1633 & 1.4 & 0.5 & 0.5 & 0.6 & 1.3 & 1.1 & 1.1 & 0.6 & snoRNA binding (IEA) \\
\hline UTP5 & orf19.7599 & 1.5 & 0.6 & 0.6 & 0.5 & 1.5 & 1.0 & 0.8 & 0.5 & \\
\hline UTP8 & orf19.5436 & 1.1 & 0.7 & 0.5 & 0.6 & 1.0 & 1.1 & 0.7 & 0.6 & tRNA binding (ISO)|snoRNA binding (IEA)|tRNA binding (IEA) \\
\hline UTP9 & orf19.6710 & 1.1 & 0.6 & 0.5 & 0.6 & 1.2 & 1.0 & 0.7 & 0.5 & snoRNA binding (IEA) \\
\hline$Y H B 4$ & orf19.7637 & 0.7 & 0.8 & 0.6 & 0.6 & 0.8 & 0.8 & 0.7 & 0.7 & nitric oxide dioxygenase activity (IMP, ISS) \\
\hline YTMI & orf19.4815 & 1.1 & 0.6 & 0.4 & 0.6 & 1.3 & 1.0 & 0.7 & 0.5 & cytoskeletal protein binding (IEA) \\
\hline YVHI & orf19.4401 & 1.3 & 0.6 & 0.6 & 0.5 & 1.6 & 1.0 & 0.8 & 0.4 & protein tyrosine/serine/threonine phosphatase activity (ISS)|protein tyrosine phosphatase activity (IEA) \\
\hline \multirow[t]{8}{*}{$Z R T 2$} & orf19.1585 & 0.7 & 0.8 & 0.5 & 0.6 & 0.6 & 1.0 & 0.6 & 0.8 & low-affinity zinc ion transmembrane transporter activity (IEA) \\
\hline & orf19.5905 & 1.1 & 0.5 & 0.5 & 0.4 & 1.6 & 0.5 & 0.9 & 0.2 & \\
\hline & orf19.2384 & 1.5 & 0.8 & 0.6 & 0.4 & 1.2 & 0.8 & 0.7 & 0.3 & \\
\hline & orf19.1642 & 2.6 & 0.5 & 0.6 & 0.3 & 2.5 & 0.9 & 0.9 & 0.3 & mRNA binding (IEA) \\
\hline & orf19.4760 & 1.6 & 0.5 & 0.4 & 0.4 & 2.0 & 0.8 & 0.8 & 0.4 & \\
\hline & orf19.2547 & 1.3 & 0.7 & 0.5 & 0.4 & 1.1 & 0.7 & 0.7 & 0.4 & \\
\hline & orf19.5353 & 1.8 & 0.7 & 0.6 & 0.5 & 1.4 & 0.8 & 0.9 & 0.4 & \\
\hline & orf19.6886 & 2.1 & 0.5 & 0.6 & 0.4 & 2.0 & 0.9 & 1.0 & 0.4 & rRNA binding (IEA) \\
\hline
\end{tabular}


Appendix C. Continued.

\begin{tabular}{|c|c|c|c|c|c|c|c|c|c|c|}
\hline & \multicolumn{8}{|c|}{ Change in fold expression ${ }^{\mathrm{a}}$} & & \\
\hline & \multicolumn{2}{|c|}{$\underline{\text { G648S }}$} & \multicolumn{2}{|c|}{$\underline{\text { G648D }}$} & \multicolumn{2}{|c|}{$\underline{\mathrm{Y} 642 \mathrm{~F}}$} & \multicolumn{2}{|c|}{$\underline{\mathrm{A} 643 \mathrm{~V}}$} & & \\
\hline \multicolumn{11}{|c|}{ CGD Name orf19 Designation Exp1 Exp2 Exp1 Exp2 Exp1 Exp2 Exp1 Exp2 Molecular function } \\
\hline orf19.5704 & 1.2 & 0.6 & 0.6 & 0.5 & 1.1 & 0.9 & 0.7 & 0.4 & & \\
\hline orf19.1708 & 2.3 & 0.5 & 0.5 & 0.4 & 2.1 & 1.1 & 0.9 & 0.4 & & \\
\hline orf19.6862 & 1.8 & 0.7 & 0.5 & 0.4 & 1.7 & 0.9 & 0.8 & 0.4 & & \\
\hline orf19.2386 & 1.7 & 0.6 & 0.6 & 0.4 & 2.0 & 1.1 & 1.0 & 0.4 & & 0 \\
\hline orf19.2320 & 1.7 & 0.4 & 0.5 & 0.3 & 1.7 & 0.9 & 0.7 & 0.4 & amino acid kinase activity (IEA)|protein kinase activity (IEA) & \\
\hline orf19.6828 & 2.0 & 0.3 & 0.4 & 0.4 & 2.0 & 1.1 & 0.9 & 0.4 & RNA binding (IEA) & \\
\hline orf19.107 & 1.5 & 0.7 & 0.6 & 0.5 & 1.4 & 1.0 & 0.7 & 0.4 & RNA helicase activity (IEA) & \\
\hline orf19.6234 & 1.8 & 0.5 & 0.6 & 0.4 & 1.5 & 0.9 & 0.9 & 0.4 & & \\
\hline orf19.2167 & 1.2 & 0.7 & 0.6 & 0.6 & 1.5 & 0.6 & 0.8 & 0.4 & & \\
\hline orf19.6297 & 1.5 & 0.7 & 0.6 & 0.6 & 1.4 & 0.9 & 0.7 & 0.4 & pseudouridine synthase activity (IEA)|pseudouridylate synthase activity (IEA) & \\
\hline orf19.6014 & 2.4 & 0.5 & 0.6 & 0.5 & 2.4 & 0.9 & 1.2 & 0.4 & & \\
\hline orf19.1082 & 1.7 & 0.5 & 0.5 & 0.5 & 1.2 & 1.0 & 0.7 & 0.4 & & \\
\hline orf19.5038 & 1.3 & 0.6 & 0.5 & 0.5 & 1.1 & 1.1 & 0.7 & 0.4 & tRNA (guanine) methyltransferase activity (IEA) & \\
\hline orf19.2830 & 1.6 & 0.6 & 0.5 & 0.4 & 1.7 & 1.0 & 0.7 & 0.4 & snoRNA binding (IEA) & \\
\hline orf19.7468 & 1.1 & 0.9 & 0.5 & 0.6 & 1.2 & 0.9 & 0.7 & 0.4 & & \\
\hline orf19.2870 & 0.7 & 1.3 & 0.6 & 0.4 & 0.7 & 0.4 & 0.7 & 0.5 & & \\
\hline orf19.2319 & 2.0 & 0.6 & 0.6 & 0.6 & 1.6 & 1.0 & 0.9 & 0.5 & RNA binding (IEA) & \\
\hline orf19.3393 & 1.4 & 0.5 & 0.5 & 0.5 & 1.5 & 1.1 & 0.7 & 0.5 & ATP-dependent RNA helicase activity (IEA) & \\
\hline orf19.7650 & 1.4 & 0.7 & 0.6 & 0.6 & 1.5 & 0.9 & 0.8 & 0.5 & & \\
\hline orf19.4793 & 1.4 & 0.5 & 0.5 & 0.5 & 1.4 & 1.1 & 0.8 & 0.5 & & \\
\hline orf19.1360 & 0.9 & 1.1 & 0.4 & 0.6 & 0.8 & 1.1 & 0.7 & 0.5 & & \\
\hline orf19.7384 & 1.5 & 0.6 & 0.5 & 0.5 & 1.5 & 0.9 & 0.9 & 0.5 & GTPase activity (IEA) & \\
\hline orf19.2362 & 1.3 & 0.7 & 0.5 & 0.6 & 1.5 & 0.9 & 0.9 & 0.5 & & \\
\hline
\end{tabular}


Appendix C. Continued.

\begin{tabular}{|c|c|c|c|c|c|c|c|c|c|}
\hline & \multicolumn{8}{|c|}{ Change in fold expression ${ }^{a}$} & \\
\hline & \multicolumn{2}{|c|}{$\underline{\text { G648S }}$} & \multicolumn{2}{|c|}{$\underline{\text { G648D }}$} & \multicolumn{2}{|c|}{$\underline{\text { Y642F }}$} & \multicolumn{2}{|c|}{$\underline{\mathrm{A} 643 \mathrm{~V}}$} & \\
\hline \multicolumn{10}{|c|}{ CGD Name orf19 Designation Exp1 Exp2 Exp1 Exp2 Exp1 Exp2 Exp1 Exp2 Molecular function } \\
\hline orf19.3463 & 1.6 & 0.6 & 0.6 & 0.5 & 1.6 & 0.9 & 0.8 & 0.5 & \\
\hline orf19.4792 & 1.4 & 0.8 & 0.5 & 0.6 & 1.2 & 0.9 & 0.8 & 0.5 & \\
\hline orf19.5039 & 1.5 & 0.8 & 0.6 & 0.6 & 1.6 & 0.9 & 0.7 & 0.5 & 3'-5'-exoribonuclease activity (IEA) \\
\hline orf19.3252 & 0.9 & 0.7 & 0.5 & 0.5 & 1.0 & 0.8 & 0.8 & 0.5 & specific RNA polymerase II transcription factor activity (IEA) \\
\hline orf19.4479 & 1.6 & 0.5 & 0.6 & 0.6 & 1.6 & 1.0 & 0.9 & 0.5 & RNA binding (IEA) \\
\hline orf19.6007 & 0.9 & 0.7 & 0.5 & 0.5 & 0.7 & 0.8 & 0.7 & 0.5 & \\
\hline orf19.3089 & 1.0 & 0.9 & 0.5 & 0.6 & 0.8 & 0.8 & 0.6 & 0.5 & \\
\hline orf19.603 & 1.2 & 0.7 & 0.6 & 0.6 & 1.2 & 1.1 & 0.7 & 0.5 & rRNA primary transcript binding (IEA)|single-stranded telomeric DNA binding (IEA) \\
\hline orf19.4342 & 1.1 & 0.7 & 0.6 & 0.6 & 0.9 & 0.8 & 0.7 & 0.5 & specific RNA polymerase II transcription factor activity (IEA)|transcription factor activity (IEA) \\
\hline orf19.6431 & 1.5 & 0.7 & 0.6 & 0.6 & 1.5 & 0.8 & 0.9 & 0.5 & \\
\hline orf19.2090 & 1.4 & 0.6 & 0.6 & 0.5 & 1.4 & 1.0 & 0.8 & 0.5 & RNA helicase activity (IEA)|snoRNA binding (IEA) \\
\hline orf19.2306 & 1.0 & 0.7 & 0.6 & 0.6 & 1.0 & 0.8 & 0.7 & 0.5 & oxidoreductase activity, acting on single donors with incorporation of molecular oxygen \\
\hline orf19.1404 & 1.3 & 0.7 & 0.6 & 0.6 & 1.5 & 0.8 & 0.7 & 0.5 & tRNA dihydrouridine synthase activity (IEA) \\
\hline orf19.7296 & 1.1 & 1.0 & 0.6 & 0.4 & 0.8 & 0.4 & 0.9 & 0.5 & \\
\hline orf19.1687 & 1.3 & 0.6 & 0.5 & 0.6 & 1.3 & 1.0 & 0.8 & 0.5 & ATP-dependent RNA helicase activity (IEA)|RNA splicing factor activity, transesterification mechanism (IEA) \\
\hline orf19.3539 & 1.1 & 0.6 & 0.5 & 0.5 & 1.5 & 1.1 & 0.7 & 0.5 & \\
\hline orf19.6874 & 1.0 & 0.9 & 0.6 & 0.6 & 0.8 & 0.8 & 0.7 & 0.5 & \\
\hline orf19.3704 & 1.1 & 0.6 & 0.6 & 0.6 & 1.1 & 0.9 & 0.7 & 0.6 & ATP-dependent RNA helicase activity (IEA) \\
\hline orf19.1959 & 1.1 & 0.7 & 0.6 & 0.6 & 1.1 & 0.8 & 0.7 & 0.6 & \\
\hline orf19.962 & 1.3 & 0.6 & 0.4 & 0.6 & 1.5 & 0.9 & 0.9 & 0.6 & \\
\hline
\end{tabular}


Appendix C. Continued.

\begin{tabular}{|c|c|c|c|c|c|c|c|c|c|}
\hline & \multicolumn{8}{|c|}{ Change in fold expression ${ }^{a}$} & \\
\hline & \multicolumn{2}{|c|}{$\underline{\text { G648S }}$} & \multicolumn{2}{|c|}{ G648D } & \multicolumn{2}{|c|}{$\underline{Y 642 F}$} & \multicolumn{2}{|c|}{$\underline{\mathrm{A} 643 \mathrm{~V}}$} & \\
\hline \multicolumn{10}{|c|}{ CGD Name orf19 Designation Exp1 Exp2 Exp1 Exp2 Exp1 Exp2 Exp1 Exp2 Molecular function } \\
\hline orf19.3303 & 1.1 & 0.6 & 0.6 & 0.5 & 1.1 & 0.9 & 0.7 & 0.6 & tRNA methyltransferase activity (IEA) \\
\hline orf19.6137 & 1.0 & 0.6 & 0.6 & 0.6 & 1.0 & 0.7 & 0.7 & 0.6 & \\
\hline orf19.7190 & 1.3 & 0.8 & 0.4 & 0.6 & 1.2 & 0.8 & 0.7 & 0.6 & oxidized purine base lesion DNA N-glycosylase activity (ISA, IEA) \\
\hline orf19.7593 & 1.3 & 0.7 & 0.6 & 0.5 & 1.2 & 0.8 & 0.8 & 0.6 & asparaginase activity (IEA) \\
\hline orf19.4940 & 1.2 & 0.7 & 0.6 & 0.6 & 1.2 & 1.0 & 0.7 & 0.6 & L-histidine transmembrane transporter activity (IEA) \\
\hline orf19.4835 & 1.2 & 0.7 & 0.6 & 0.6 & 1.7 & 0.8 & 0.8 & 0.6 & snoRNA binding (IEA) \\
\hline orf19.5802 & 1.3 & 0.6 & 0.6 & 0.5 & 1.2 & 0.7 & 0.8 & 0.6 & \\
\hline orf19.4375 & 1.0 & 0.7 & 0.6 & 0.6 & 1.1 & 0.9 & 0.8 & 0.6 & \\
\hline orf19.6723 & 1.3 & 0.7 & 0.6 & 0.6 & 1.1 & 0.7 & 0.8 & 0.6 & \\
\hline orf19.2527 & 1.4 & 0.5 & 0.6 & 0.6 & 1.6 & 0.9 & 0.8 & 0.6 & \\
\hline orf19.6580 & 0.9 & 0.7 & 0.6 & 0.5 & 1.0 & 0.7 & 0.8 & 0.6 & \\
\hline orf19.6730 & 1.8 & 0.5 & 0.6 & 0.6 & 1.4 & 0.9 & 1.1 & 0.6 & \\
\hline orf19.6578 & 0.9 & 0.9 & 0.5 & 0.5 & 0.8 & 0.8 & 0.7 & 0.6 & neurotransmitter transporter activity (ISS)|inorganic phosphate transmembrane transporter activity (IEA) \\
\hline orf19.7422 & 1.4 & 0.6 & 0.6 & 0.5 & 1.5 & 0.8 & 0.8 & 0.6 & RNA binding (IEA) \\
\hline orf19.2319 & 1.4 & 1.0 & 0.5 & 0.4 & 2.0 & 0.9 & 1.3 & 0.6 & RNA binding \\
\hline orf19.6766 & 1.3 & 0.5 & 0.5 & 0.6 & 1.3 & 1.0 & 0.8 & 0.6 & RNA binding (IEA) \\
\hline orf19.1697 & 1.2 & 0.8 & 0.6 & 0.5 & 1.3 & 0.8 & 0.8 & 0.6 & \\
\hline orf19.254 & 1.0 & 0.9 & 0.5 & 0.5 & 1.1 & 0.8 & 0.7 & 0.6 & \\
\hline orf19.443 & 1.3 & 0.6 & 0.6 & 0.6 & 1.1 & 0.9 & 0.9 & 0.6 & DNA-directed RNA polymerase activity (IEA) \\
\hline orf19.7107 & 1.3 & 0.5 & 0.6 & 0.6 & 1.5 & 0.9 & 1.0 & 0.6 & \\
\hline orf19.4666 & 0.9 & 0.8 & 0.6 & 0.5 & 0.7 & 0.8 & 0.7 & 0.6 & \\
\hline orf19.3270 & 0.9 & 0.9 & 0.6 & 0.6 & 0.7 & 0.8 & 0.7 & 0.6 & 1,3-beta-glucan synthase activity (IEA) \\
\hline orf19.2045 & 1.0 & 0.7 & 0.6 & 0.6 & 1.0 & 0.9 & 0.8 & 0.6 & diacylglycerol kinase activity (IEA) \\
\hline
\end{tabular}


Appendix C. Continued.

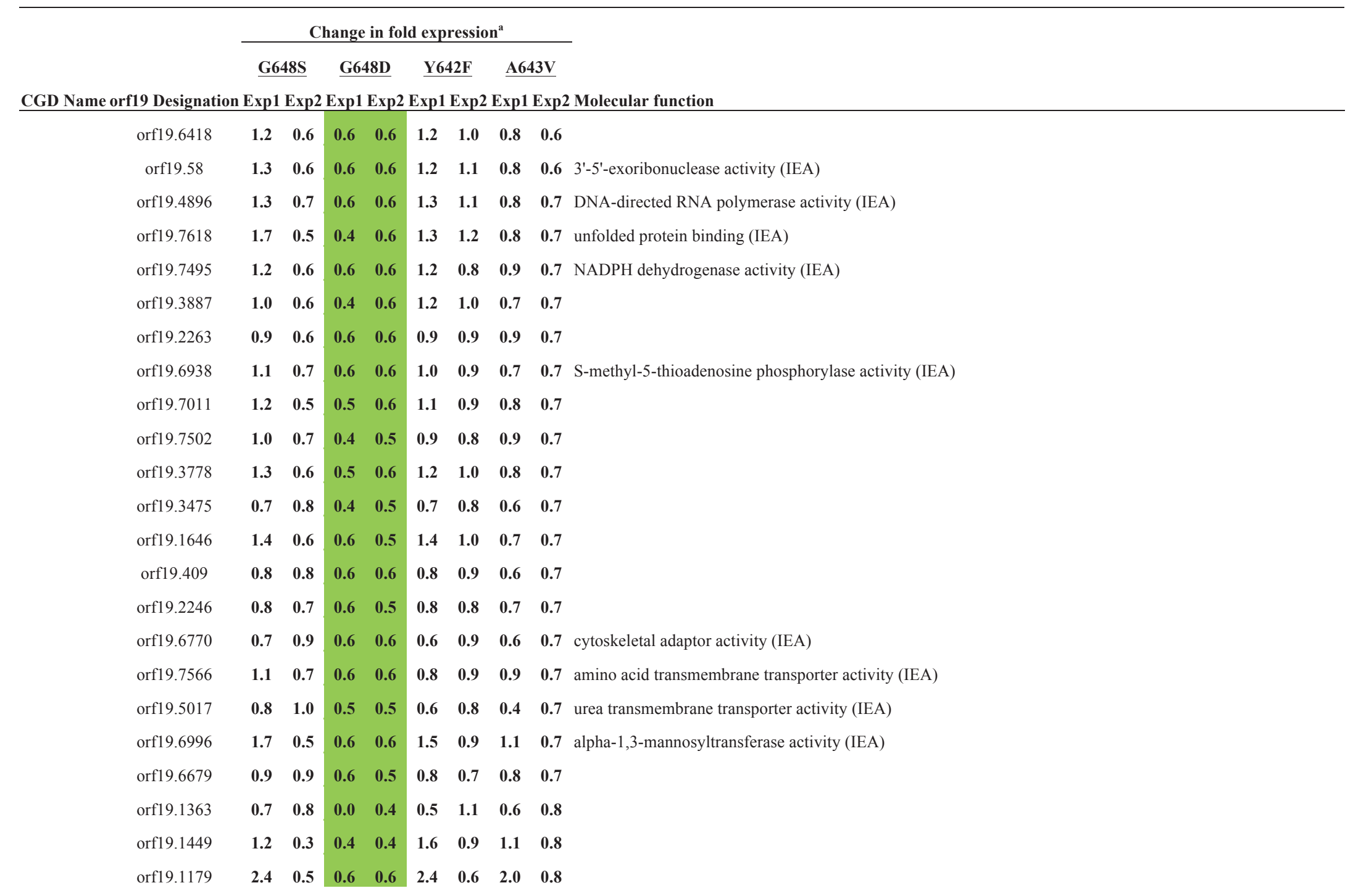


Appendix C. Continued.

\begin{tabular}{|c|c|c|c|c|c|c|c|c|c|}
\hline & & \multicolumn{7}{|c|}{ Change in fold expression ${ }^{a}$} & \\
\hline & & \multicolumn{2}{|c|}{$\underline{\text { G648S }}$} & \multicolumn{2}{|c|}{ G648D } & \multicolumn{2}{|c|}{$\underline{Y 642 F}$} & $\underline{\mathrm{A} 643 \mathrm{~V}}$ & \\
\hline \multicolumn{10}{|c|}{ CGD Name orf19 Designation Exp1 Exp2 Exp1 Exp2 Exp1 Exp2 Exp1 Exp2 Molecular function } \\
\hline & orf19.4266 & 1.0 & 0.8 & 0.6 & 0.3 & 1.1 & 0.8 & $0.4 \quad 0.8$ & \\
\hline & orf19.2959.1 & 0.6 & 0.9 & 0.5 & 0.6 & 0.6 & 0.8 & $0.6 \quad 0.8$ & \\
\hline & orf19.3894 & 0.9 & 0.9 & 0.5 & 0.6 & 1.0 & 1.1 & 0.9 & \\
\hline & orf19.670.2 & 0.8 & 0.4 & 0.3 & 0.4 & 0.7 & 0.5 & $1.5 \quad 0.9$ & \\
\hline ARAl & orf19.2172 & 0.6 & 1.2 & 1.3 & 1.0 & 0.6 & 0.4 & $0.8 \quad 0.7$ & D-arabinose 1-dehydrogenase $[\mathrm{NAD}(\mathrm{P})+]$ activity (IDA) \\
\hline$C I P 1$ & orf19.113 & 0.7 & 2.3 & 1.1 & 0.7 & 0.5 & 0.5 & 0.7 & \\
\hline CIRTAB & orf19.2839 & 0.8 & 1.4 & 1.6 & 1.3 & 0.6 & 0.6 & $1.4 \quad 1.3$ & \\
\hline GACl & orf19.7053 & 0.7 & 1.9 & 1.2 & 0.7 & 0.6 & 0.6 & $0.6 \quad 0.6$ & protein phosphatase type 1 regulator activity (IEA) \\
\hline$I F C 3$ & orf19.3749 & 0.6 & 1.7 & 1.4 & 1.1 & 0.6 & 0.6 & 1.0 & oligopeptide transporter activity (IGI, ISS) \\
\hline IFEI & orf19.769 & 0.5 & 1.7 & 0.8 & 0.8 & 0.4 & 0.6 & $0.4 \quad 0.8$ & \\
\hline$L I P 1$ & orf19.4821 & 0.3 & 0.9 & 1.0 & 0.8 & 0.6 & 0.4 & 0.9 & lipase activity (IDA) \\
\hline MIHI & orf19.3071 & 0.7 & 0.9 & 0.7 & 0.6 & 0.6 & 0.6 & 0.8 & protein tyrosine phosphatase activity (IEA) \\
\hline OPT7 & orf19.5673 & 0.7 & 1.4 & 0.4 & 0.4 & 0.3 & 0.6 & 0.6 & oligopeptide transporter activity (ISS) \\
\hline$P R C 2$ & orf19.4135 & 0.6 & 2.2 & 0.9 & 0.7 & 0.4 & 0.5 & 0.6 & \\
\hline PSA2 & orf19.4943 & 0.6 & 1.4 & 0.9 & 0.7 & 0.5 & 0.5 & 0.8 & mannose-1-phosphate guanylyltransferase activity (IDA, IGI, ISS) \\
\hline$R B T 7$ & orf19.2681 & 0.7 & 1.2 & 0.8 & 0.4 & 0.4 & 0.4 & 0.7 & ribonuclease activity (ISS) \\
\hline SAP10 & orf19.3839 & 0.5 & 1.3 & 0.8 & 0.5 & 0.5 & 0.5 & 0.6 & \\
\hline \multirow[t]{6}{*}{ SKN2 } & orf19.348 & 0.7 & 1.1 & 0.6 & 0.7 & 0.5 & 0.6 & 0.8 & \\
\hline & orf19.5775 & 0.9 & 1.2 & 1.1 & 0.8 & 0.6 & 0.4 & 0.6 & \\
\hline & orf19.7311 & 0.6 & 1.0 & 0.7 & 0.8 & 0.6 & 0.6 & 0.6 & \\
\hline & orf19.6795 & 0.7 & 0.8 & 0.5 & 0.7 & 0.5 & 0.5 & $0.7 \quad 0.7$ & transcription regulator activity (IEA) \\
\hline & orf19.3758 & 0.4 & 0.8 & 0.1 & 0.8 & 0.6 & 0.5 & 0.3 & \\
\hline & orf19.1484 & 0.7 & 0.8 & 0.9 & 1.2 & 0.6 & 0.5 & 0.7 & \\
\hline
\end{tabular}


Appendix C. Continued.

\begin{tabular}{|c|c|c|c|c|c|c|c|c|c|c|}
\hline & & \multicolumn{8}{|c|}{ Change in fold expression ${ }^{\mathrm{a}}$} & \\
\hline & & \multicolumn{2}{|c|}{$\underline{\text { G648S }}$} & \multicolumn{2}{|c|}{$\underline{\text { G648D }}$} & \multicolumn{2}{|c|}{$\underline{\mathrm{Y} 642 \mathrm{~F}}$} & \multicolumn{2}{|c|}{$\underline{\mathrm{A} 643 \mathrm{~V}}$} & \\
\hline \multicolumn{11}{|c|}{ CGD Name orf19 Designation Exp1 Exp2 Exp1 Exp2 Exp1 Exp2 Exp1 Exp2 Molecular function } \\
\hline & orf19.1258 & 0.5 & 1.4 & 1.0 & 0.6 & 0.4 & 0.5 & & 0.7 & \\
\hline & orf19.5449 & 0.8 & 1.0 & 0.8 & 0.6 & 0.6 & 0.6 & 0.7 & 0.7 & \\
\hline & orf19.1600 & 0.7 & 0.9 & 0.9 & 0.5 & 0.5 & 0.6 & 0.6 & 0.7 & \\
\hline & orf19.4768 & 1.0 & 0.5 & 0.9 & 1.8 & 0.4 & 0.5 & 0.7 & 0.8 & \\
\hline & orf19.2737 & 0.5 & 1.2 & 0.8 & 0.7 & 0.5 & 0.6 & 0.5 & 0.8 & \\
\hline & orf19.4349 & 0.9 & 0.7 & 0.5 & 0.9 & 0.5 & 0.6 & 0.4 & 0.8 & \\
\hline & orf19.2030 & 0.7 & 1.2 & 0.8 & 0.7 & 0.6 & 0.6 & 0.8 & 0.8 & \\
\hline & orf19.1887 & 0.6 & 1.1 & 0.9 & 0.7 & 0.6 & 0.6 & 0.6 & 0.8 & sterol esterase activity (IEA) \\
\hline & orf19.764 & 0.1 & 1.0 & 0.6 & 0.8 & 0.6 & 0.1 & 0.6 & 0.8 & \\
\hline & orf19.6017 & 1.2 & 0.9 & 0.8 & 0.7 & 0.6 & 0.6 & 1.0 & 0.9 & \\
\hline & orf19.1159 & 0.8 & 0.9 & 0.6 & 0.7 & 0.6 & 0.6 & 1.0 & 0.9 & homoserine O-acetyltransferase activity (IEA) \\
\hline & orf19.4530.1 & 0.4 & 2.1 & 0.9 & 0.9 & 0.4 & 0.6 & 0.5 & 0.9 & \\
\hline & orf19.6307 & 0.6 & 1.1 & 0.5 & 1.2 & 0.4 & 0.6 & 0.8 & 1.1 & \\
\hline & orf19.5527 & 0.6 & 0.8 & 1.0 & 0.9 & 0.6 & 0.5 & 1.4 & 1.2 & endoribonuclease activity (IEA) \\
\hline & orf19.247 & 0.7 & 0.7 & 1.1 & 1.0 & 0.6 & 0.4 & 0.7 & 1.4 & \\
\hline & orf19.4055 & 0.5 & 0.8 & 0.6 & 1.0 & 0.3 & 0.4 & 1.7 & 1.8 & \\
\hline ASR3 & orf19.842 & 0.8 & 1.2 & 0.8 & 0.5 & 0.8 & 0.5 & 0.6 & 0.5 & \\
\hline BMT4 & orf19.5612 & 0.8 & 1.4 & 1.0 & 0.4 & 0.7 & 0.6 & 0.6 & 0.6 & \\
\hline СНT3 & orf19.7586 & 0.7 & 0.8 & 0.6 & 0.7 & 0.6 & 0.7 & 0.6 & 0.6 & chitinase activity (IDA, IGI, ISS)|endochitinase activity (IEA) \\
\hline CLA4 & orf19.4890 & 0.7 & 0.8 & 0.5 & 0.8 & 0.8 & 0.6 & 0.5 & 0.4 & protein serine/threonine kinase activity (ISS) \\
\hline$C R Z 2$ & orf19.2356 & 0.8 & 1.9 & 1.0 & 0.5 & 0.8 & 0.7 & 0.3 & 0.4 & transcription factor activity (ISS) \\
\hline CSP37 & orf19.2531 & 0.6 & 0.9 & 0.7 & 0.6 & 0.7 & 0.5 & 0.6 & 0.6 & \\
\hline$F C Y 21$ & orf19.1357 & 1.2 & 1.1 & 0.8 & 0.8 & 0.8 & 0.7 & 0.6 & 0.6 & drug membrane transporter activity \\
\hline
\end{tabular}




\begin{tabular}{|c|c|c|c|c|c|c|c|c|c|c|}
\hline & & \multicolumn{8}{|c|}{ Change in fold expression ${ }^{a}$} & \\
\hline & & \multicolumn{2}{|c|}{$\underline{\text { G648S }}$} & \multicolumn{2}{|c|}{$\underline{\text { G648D }}$} & \multicolumn{2}{|c|}{$\underline{\mathbf{Y} 642 \mathrm{~F}}$} & \multicolumn{2}{|c|}{$\underline{\mathrm{A} 643 \mathrm{~V}}$} & \\
\hline \multicolumn{11}{|c|}{ CGD Name orf19 Designation Exp1 Exp2 Exp1 Exp2 Exp1 Exp2 Exp1 Exp2 Molecular function } \\
\hline FGR6 & orf19.5315 & 0.8 & 0.9 & 0.8 & 0.6 & 0.7 & 0.5 & 0.6 & 0.5 & \\
\hline GIT1 & orf19.1979 & 0.8 & 1.3 & 0.9 & 0.7 & 0.8 & 0.8 & 0.6 & 0.6 & inorganic phosphate transmembrane transporter activity (ISS)|phospholipid transporter activity (IEA) \\
\hline GNP3 & orf19.7565 & 1.0 & 0.8 & 1.0 & 0.7 & 0.8 & 0.8 & 0.6 & 0.6 & amino acid transmembrane transporter activity (IEA) \\
\hline KRE1 & orf19.4377 & 1.2 & 1.2 & 0.7 & 0.6 & 1.0 & 1.0 & 0.6 & 0.5 & \\
\hline$M E A 1$ & orf19.5919 & 1.1 & 1.0 & 0.7 & 0.6 & 0.9 & 0.8 & 0.6 & 0.6 & \\
\hline MRRI & orf19.7372 & 1.1 & 1.0 & 0.7 & 0.6 & 1.1 & 0.9 & 0.6 & 0.5 & specific RNA polymerase II transcription factor activity (IMP)|sequence-specific DNA binding (IEA) \\
\hline PHO84 & orf19.655 & 0.8 & 1.2 & 0.9 & 0.6 & 0.8 & 0.9 & 0.6 & 0.6 & inorganic phosphate transmembrane transporter activity (ISS) \\
\hline$R C L 1$ & orf19.1886 & 0.9 & 0.7 & 0.5 & 0.7 & 0.8 & 1.0 & 0.6 & 0.6 & RNA-3'-phosphate cyclase activity (IEA)|enzyme activator activity (IEA) \\
\hline SFU1 & orf19.4869 & 1.1 & 1.1 & 0.8 & 0.6 & 0.9 & 0.9 & 0.6 & 0.6 & DNA binding (IGI)|transcription factor activity (IGI, IMP, ISS) \\
\hline$T E C 1$ & orf19.5908 & 1.0 & 0.9 & 0.7 & 0.4 & 0.9 & 0.8 & 0.5 & 0.6 & specific RNA polymerase II transcription factor activity (IMP, ISS)|transcription factor activity (ISS) \\
\hline \multirow[t]{13}{*}{ XUT1 } & orf19.2882 & 0.9 & 1.1 & 1.0 & 0.4 & 0.9 & 0.5 & 0.5 & 0.5 & nucleobase:cation symporter activity (ISS) \\
\hline & orf19.5826 & 0.5 & 0.8 & 0.6 & 0.7 & 0.6 & 0.4 & 0.5 & 0.4 & L-gamma-aminobutyric acid transmembrane transporter activity (IEA) \\
\hline & orf19.6277 & 1.0 & 1.6 & 0.8 & 0.3 & 0.9 & 0.5 & 0.3 & 0.4 & \\
\hline & orf19.7590 & 0.9 & 0.9 & 0.7 & 0.5 & 1.1 & 0.5 & 0.6 & 0.5 & \\
\hline & orf19.5775 & 0.8 & 0.9 & 1.2 & 0.4 & 1.0 & 0.5 & 0.6 & 0.6 & \\
\hline & orf19.3470 & 1.4 & 0.6 & 0.9 & 0.3 & 1.5 & 0.5 & 0.3 & 0.3 & nitrogenase (flavodoxin) activity (ISS) \\
\hline & orf19.5070 & 0.8 & 1.2 & 0.7 & 0.3 & 0.7 & 0.6 & 0.5 & 0.5 & \\
\hline & orf19.3988 & 0.9 & 1.1 & 0.4 & 0.7 & 1.1 & 0.6 & 0.5 & 0.6 & \\
\hline & orf19.750 & 0.7 & 1.4 & 0.6 & 0.7 & 0.7 & 0.6 & 0.6 & 0.6 & \\
\hline & orf19.5872 & 1.2 & 1.0 & 0.9 & 0.7 & 0.4 & 0.7 & 0.6 & 0.4 & general RNA polymerase II transcription factor activity (IEA) \\
\hline & orf19.48 & 1.8 & 0.9 & 1.0 & 0.8 & 1.4 & 0.7 & 0.4 & 0.4 & ribonuclease $\mathrm{P}$ activity (IEA) \\
\hline & orf19.5910 & 1.0 & 0.8 & 1.0 & 0.6 & 1.0 & 0.7 & 0.6 & 0.6 & histone acetyltransferase activity (IEA)|methylated histone residue binding (IEA) \\
\hline & orf19.1656 & 0.4 & 1.5 & 1.3 & 0.7 & 0.6 & 0.7 & 0.6 & 0.6 & phosphatidylinositol binding (IEA) \\
\hline
\end{tabular}


Appendix C. Continued.

\begin{tabular}{|c|c|c|c|c|c|c|c|c|c|}
\hline & & \multicolumn{7}{|c|}{ Change in fold expression ${ }^{\mathrm{a}}$} & \\
\hline & & \multicolumn{2}{|c|}{$\underline{\text { G648S }}$} & \multicolumn{2}{|c|}{ G648D } & \multicolumn{2}{|c|}{$\underline{\mathrm{Y} 642 \mathrm{~F}}$} & $\underline{\mathrm{A} 643 \mathrm{~V}}$ & \\
\hline \multicolumn{10}{|c|}{ CGD Name orf19 Designation Exp1 Exp2 Exp1 Exp2 Exp1 Exp2 Exp1 Exp2 Molecular function } \\
\hline & orf19.2824 & 0.8 & 0.9 & 0.7 & 0.7 & 0.8 & 0.7 & $0.6 \quad 0.6$ & double-stranded DNA binding (IDA)|specific transcriptional repressor activity (IGI) \\
\hline & orf19.2604 & 1.3 & 0.7 & 0.7 & 0.4 & 1.3 & 0.7 & 0.5 & \\
\hline & orf19.2231 & 1.1 & 0.9 & 0.8 & 0.8 & 1.2 & 0.7 & 0.6 & \\
\hline & orf19.2547 & 1.3 & 0.6 & 0.7 & 0.5 & 1.1 & 0.8 & $\begin{array}{ll}0.6 & 0.4\end{array}$ & \\
\hline & orf19.4143 & 1.5 & 0.6 & 0.8 & 0.4 & 1.2 & 0.8 & 0.6 & \\
\hline & orf19.3170 & 1.5 & 0.6 & 0.8 & 0.7 & 1.5 & 0.8 & 0.6 & \\
\hline & orf19.6976 & 0.9 & 1.0 & 0.8 & 0.7 & 0.9 & 0.8 & 0.6 & folic acid transporter activity (ISS) \\
\hline & orf19.5326 & 1.3 & 1.0 & 0.7 & 0.6 & 1.0 & 0.9 & 0.6 & transcription factor activity (ISS)|transcription corepressor activity (IEA) \\
\hline & orf19.3481 & 1.0 & 0.7 & 0.5 & 0.7 & 0.9 & 0.9 & 0.6 & \\
\hline & orf19.3831 & 1.7 & 1.2 & 0.9 & 0.7 & 1.5 & 1.0 & 0.6 & \\
\hline & orf19.6416 & 1.0 & 1.2 & 0.7 & 0.8 & 0.8 & 1.0 & 0.6 & \\
\hline & orf19.6662 & 1.1 & 0.9 & 0.9 & 0.7 & 1.2 & 1.0 & 0.6 & ubiquinone binding (IEA) \\
\hline & orf19.5128 & 1.0 & 1.1 & 0.9 & 1.2 & 1.1 & 1.0 & 0.6 & RNA binding (IEA) \\
\hline & orf19.1772 & 1.8 & 1.1 & 0.6 & 0.8 & 1.7 & 1.2 & 0.5 & \\
\hline & orf19.69 & 0.6 & 1.0 & 0.7 & 0.9 & 0.5 & 1.3 & 0.5 & \\
\hline & orf19.6586 & 0.9 & 1.7 & 0.7 & 0.6 & 1.0 & 1.3 & 0.4 & \\
\hline & orf19.4563 & 1.1 & 0.6 & 0.5 & 0.8 & 1.0 & 1.5 & 0.5 & \\
\hline & orf19.1117 & 1.1 & 5.5 & 0.1 & 0.7 & 0.8 & 2.1 & 0.1 & formate dehydrogenase activity (ISS) \\
\hline & orf19.2442 & 0.5 & 0.5 & 0.3 & 0.5 & 0.5 & 0.7 & 0.5 & \\
\hline & orf19.2163 & 0.3 & 0.5 & 0.6 & 0.7 & 0.4 & 0.3 & 0.7 & \\
\hline HAL21 & orf19.99 & 0.4 & 0.4 & 0.4 & 1.2 & 0.2 & 0.4 & 0.4 & $3^{\prime}\left(2^{\prime}\right), 5^{\prime}$-bisphosphate nucleotidase activity (IEA) \\
\hline$A F G 1$ & orf19.4743 & 0.8 & 0.6 & 0.5 & 0.5 & 0.6 & 0.5 & 0.9 & ATPase activity (IEA) \\
\hline$H G T 1$ & orf19.4527 & 0.9 & 0.5 & 0.2 & 0.5 & 0.6 & 0.5 & 0.6 & glucose transmembrane transporter activity (IGI, ISS)|sugar transmembrane transporter activity (ISS) \\
\hline
\end{tabular}


Appendix C. Continued.

\begin{tabular}{|c|c|c|c|c|c|c|c|c|c|c|}
\hline & & \multicolumn{8}{|c|}{ Change in fold expression ${ }^{\mathrm{a}}$} & \\
\hline & & \multicolumn{2}{|c|}{$\underline{\text { G648S }}$} & \multicolumn{2}{|c|}{ G648D } & \multicolumn{2}{|c|}{$\underline{Y 642 F}$} & \multicolumn{2}{|c|}{$\underline{\mathrm{A} 643 \mathrm{~V}}$} & \\
\hline \multicolumn{11}{|c|}{ CGD Name orf19 Designation Exp1 Exp2 Exp1 Exp2 Exp1 Exp2 Exp1 Exp2 Molecular function } \\
\hline HSP30 & orf19.4526 & 0.6 & 2.3 & 0.3 & 0.4 & 0.4 & 0.4 & 0.5 & 0.7 & \\
\hline IFF11 & orf19.5399 & 0.6 & 0.8 & 0.6 & 0.5 & 0.5 & 0.6 & 0.8 & 1.0 & \\
\hline OPT7 & orf19.5673 & 0.7 & 1.4 & 0.4 & 0.4 & 0.3 & 0.6 & 0.6 & 0.7 & oligopeptide transporter activity (ISS) \\
\hline$S A P 3$ & orf19.6001 & 0.6 & 1.4 & 0.6 & 0.6 & 0.5 & 0.6 & 0.6 & 1.1 & aspartic-type endopeptidase activity (IDA, ISS)|drug binding (IDA,ISS) \\
\hline \multirow[t]{2}{*}{ SIT1 } & orf19.2179 & 1.0 & 0.7 & 0.4 & 0.5 & 0.6 & 0.6 & 0.8 & 0.5 & ferrichrome transporter activity (IGI, IMP)|siderophore-iron transmembrane transporter activity (ISS) \\
\hline & orf19.675 & 0.5 & 1.5 & 0.6 & 0.6 & 0.4 & 0.6 & 0.4 & 0.7 & \\
\hline$A R G 11$ & orf19.5926 & 1.0 & 0.9 & 0.4 & 0.5 & 1.3 & 0.8 & 0.5 & 0.3 & L-ornithine transmembrane transporter activity (IEA) \\
\hline$C C C 1$ & orf19.6948 & 0.8 & 0.9 & 0.4 & 0.4 & 0.7 & 0.9 & 0.3 & 0.4 & ferrous iron transmembrane transporter activity (IEA) \\
\hline $\mathrm{CHO} 2$ & orf19.169 & 1.1 & 0.7 & 0.6 & 0.5 & 0.9 & 0.8 & 0.6 & 0.5 & methylene-fatty-acyl-phospholipid synthase activity (IEA) \\
\hline CUP9 & orf19.6514 & 1.0 & 1.1 & 0.6 & 0.5 & 0.9 & 0.9 & 0.6 & 0.5 & chromatin binding (IEA)|sequence-specific DNA binding (IEA) \\
\hline DIM1 & orf19.5010 & 1.6 & 0.6 & 0.4 & 0.4 & 1.9 & 1.2 & 0.6 & 0.3 & rRNA (adenine-N6,N6-)-dimethyltransferase activity (IEA) \\
\hline ELP3 & orf19.7387 & 1.1 & 0.7 & 0.6 & 0.6 & 1.1 & 1.0 & 0.6 & 0.5 & RNA polymerase II transcription elongation factor activity (IEA) \\
\hline$F D H 1$ & orf19.638 & 0.8 & 1.4 & 0.6 & 0.4 & 0.7 & 0.7 & 0.5 & 0.5 & formate dehydrogenase activity (ISS) \\
\hline FET3 & orf19.4211 & 0.9 & 0.5 & 0.3 & 0.4 & 0.7 & 0.7 & 0.6 & 0.5 & ferroxidase activity (IGI, IMP) \\
\hline FRE30 & orf19.6140 & 1.4 & 0.5 & 0.3 & 0.3 & 1.2 & 0.6 & 0.6 & 0.3 & \\
\hline FRE7 & orf19.6139 & 1.1 & 0.6 & 0.2 & 0.4 & 0.8 & 0.9 & 0.5 & 0.5 & ferric-chelate reductase activity (IEA) \\
\hline HCMI & orf19.4853 & 0.8 & 0.8 & 0.5 & 0.6 & 0.6 & 0.8 & 0.6 & 0.6 & sequence-specific DNA binding (IEA) \\
\hline$H M X 1$ & orf19.6073 & 1.1 & 0.8 & 0.3 & 0.5 & 1.0 & 1.1 & 0.4 & 0.4 & heme oxygenase (decyclizing) activity (IDA, IMP, ISS) \\
\hline HSP12 & orf19.3160 & 0.6 & 1.2 & 0.6 & 0.4 & 0.6 & 0.7 & 0.5 & 0.6 & \\
\hline NOG2 & orf19.5732 & 1.4 & 0.6 & 0.6 & 0.4 & 1.4 & 0.7 & 0.6 & 0.2 & \\
\hline NOP14 & orf19.5959 & 2.2 & 0.6 & 0.6 & 0.4 & 2.9 & 0.7 & 0.6 & 0.2 & snoRNA binding (IEA) \\
\hline PGA55 & orf19.207 & 0.9 & 0.7 & 0.5 & 0.5 & 0.6 & 0.8 & 0.5 & 0.5 & \\
\hline RLII & orf19.3034 & 1.1 & 0.7 & 0.6 & 0.5 & 1.1 & 0.9 & 0.6 & 0.5 & ATP binding (ISS)|iron ion binding (IEA) \\
\hline
\end{tabular}


Appendix C. Continued.

\begin{tabular}{|c|c|c|c|c|c|c|c|c|c|}
\hline & & \multicolumn{7}{|c|}{ Change in fold expression ${ }^{a}$} & \\
\hline & & \multicolumn{2}{|c|}{$\underline{\text { G648S }}$} & \multicolumn{2}{|c|}{ G648D } & \multicolumn{2}{|c|}{$\underline{Y 642 F}$} & $\underline{\mathrm{A} 643 \mathrm{~V}}$ & \\
\hline \multicolumn{10}{|c|}{ CGD Name orf19 Designation Exp1 Exp2 Exp1 Exp2 Exp1 Exp2 Exp1 Exp2 Molecular function } \\
\hline SEN2 & orf19.2735 & 1.3 & 0.5 & 0.4 & 0.3 & 1.2 & 1.1 & $0.5 \quad 0.3$ & \\
\hline$S M M 1$ & orf19.1362 & 1.4 & 0.6 & 0.5 & 0.3 & 1.4 & 0.7 & $0.5 \quad 0.3$ & tRNA dihydrouridine synthase activity (IEA) \\
\hline$S P B 4$ & orf19.6298 & 1.5 & 0.6 & 0.5 & 0.5 & 1.6 & 1.1 & 0.6 & ATP-dependent RNA helicase activity (IEA) \\
\hline \multirow{20}{*}{ UTP18 } & orf19.7154 & 1.2 & 0.6 & 0.4 & 0.5 & 1.1 & 1.1 & 0.6 & \\
\hline & orf19.4445 & 1.0 & 0.4 & 0.2 & 0.1 & 1.2 & 0.5 & 0.30 .1 & \\
\hline & orf19.6873.1 & 2.2 & 0.8 & 0.3 & 0.2 & 1.6 & 0.9 & 0.5 & electron carrier activity (IEA)|iron ion binding (IEA) \\
\hline & orf19.1091 & 1.5 & 0.4 & 0.4 & 0.3 & 1.9 & 0.5 & $\begin{array}{ll}0.6 & 0.2\end{array}$ & RNA binding (IEA) \\
\hline & orf19.5049 & 1.9 & 0.5 & 0.5 & 0.5 & 1.4 & 0.9 & $\begin{array}{ll}0.4 & 0.2\end{array}$ & \\
\hline & orf19.5207 & 1.2 & 0.6 & 0.4 & 0.5 & 1.1 & 1.1 & 0.3 & \\
\hline & orf19.5500 & 1.7 & 0.6 & 0.5 & 0.4 & 1.9 & 1.2 & 0.5 & \\
\hline & orf19.2934 & 1.6 & 0.5 & 0.4 & 0.4 & 1.8 & 0.7 & 0.6 & \\
\hline & orf19.341 & 1.0 & 1.0 & 0.2 & 0.2 & 0.6 & 0.7 & 0.2 & drug transporter activity (ISS) \\
\hline & orf19.7077 & 1.0 & 0.4 & 0.2 & 0.3 & 0.7 & 0.6 & 0.3 & ferric-chelate reductase activity (IEA) \\
\hline & orf19.5843 & 0.7 & 0.7 & 0.3 & 0.4 & 0.7 & 0.8 & 0.4 & \\
\hline & orf19.5020 & 1.2 & 0.7 & 0.4 & 0.3 & 1.2 & 0.9 & 0.5 & \\
\hline & orf19.1774 & 1.0 & 1.0 & 0.3 & 0.3 & 0.5 & 0.9 & 0.3 & \\
\hline & orf19.4273 & 1.6 & 0.7 & 0.5 & 0.4 & 1.3 & 1.1 & 0.5 & \\
\hline & orf19.3448 & 0.9 & 1.0 & 0.2 & 0.2 & 0.9 & 0.5 & 0.4 & \\
\hline & orf19.2724 & 0.6 & 1.3 & 0.4 & 0.3 & 0.6 & 0.7 & 0.3 & \\
\hline & orf19.494 & 1.3 & 0.5 & 0.5 & 0.5 & 1.5 & 0.9 & 0.6 & RNA binding (IEA) \\
\hline & orf19.3088 & 1.3 & 0.5 & 0.4 & 0.4 & 1.3 & 1.0 & 0.6 & transcription factor activity (IEA) \\
\hline & orf19.2604 & 1.3 & 0.5 & 0.4 & 0.4 & 1.4 & 0.8 & $\begin{array}{ll}0.6 & 0.4\end{array}$ & \\
\hline & orf19.1771 & 1.3 & 0.8 & 0.4 & 0.4 & 1.3 & 0.6 & 0.40 .4 & \\
\hline
\end{tabular}


Appendix C. Continued.

\begin{tabular}{|c|c|c|c|c|c|c|c|c|c|}
\hline & \multicolumn{8}{|c|}{ Change in fold expression ${ }^{a}$} & \\
\hline & \multicolumn{2}{|c|}{$\underline{\text { G648S }}$} & \multicolumn{2}{|c|}{ G648D } & \multicolumn{2}{|c|}{$\underline{\mathrm{Y} 642 \mathrm{~F}}$} & \multicolumn{2}{|c|}{$\underline{\mathrm{A} 643 \mathrm{~V}}$} & \\
\hline \multicolumn{10}{|c|}{ CGD Name orf19 Designation Exp1 Exp2 Exp1 Exp2 Exp1 Exp2 Exp1 Exp2 Molecular function } \\
\hline orf19.6660 & 0.6 & 1.2 & 0.5 & 0.3 & 0.7 & 0.6 & 0.3 & 0.4 & \\
\hline orf19.2018 & 1.1 & 0.6 & 0.5 & 0.4 & 1.0 & 0.8 & 0.5 & 0.4 & \\
\hline orf19.22 & 0.8 & 0.7 & 0.4 & 0.3 & 0.6 & 0.7 & 0.4 & 0.4 & \\
\hline orf19.6899 & 1.0 & 2.7 & 0.4 & 0.5 & 0.6 & 0.9 & 0.2 & 0.4 & \\
\hline orf19.1287 & 1.7 & 0.8 & 0.6 & 0.5 & 1.5 & 1.1 & 0.6 & 0.4 & \\
\hline orf19.3759 & 0.9 & 0.8 & 0.4 & 0.4 & 1.3 & 0.7 & 0.5 & 0.4 & RNA polymerase II transcription elongation factor activity (IEA) \\
\hline orf19.4634 & 1.6 & 0.6 & 0.6 & 0.6 & 1.2 & 1.0 & 0.6 & 0.4 & \\
\hline orf19.5431 & 0.7 & 0.7 & 0.3 & 0.3 & 0.5 & 0.7 & 0.4 & 0.4 & \\
\hline orf19.3048 & 0.6 & 0.8 & 0.6 & 0.5 & 0.8 & 0.5 & 0.6 & 0.5 & \\
\hline orf19.1388 & 1.5 & 0.5 & 0.5 & 0.5 & 1.6 & 1.1 & 0.5 & 0.5 & \\
\hline orf19.2330 & 1.2 & 0.5 & 0.4 & 0.5 & 1.2 & 0.9 & 0.5 & 0.5 & snoRNA binding (IEA) \\
\hline orf19.7088 & 1.3 & 0.7 & 0.5 & 0.6 & 1.4 & 1.1 & 0.6 & 0.5 & single-stranded telomeric DNA binding (IEA) \\
\hline orf19.4161 & 0.9 & 0.8 & 0.5 & 0.6 & 1.4 & 0.6 & 0.6 & 0.5 & \\
\hline orf19.4900 & 0.8 & 0.7 & 0.5 & 0.4 & 0.7 & 0.5 & 0.6 & 0.5 & alpha-1,3-mannosyltransferase activity (IEA) \\
\hline orf19.7085 & 0.6 & 1.0 & 0.5 & 0.4 & 0.8 & 0.6 & 0.5 & 0.5 & \\
\hline orf19.3335 & 0.7 & 0.8 & 0.6 & 0.6 & 0.9 & 0.7 & 0.6 & 0.5 & \\
\hline orf19.6751 & 1.2 & 0.8 & 0.6 & 0.6 & 1.3 & 1.0 & 0.6 & 0.5 & tRNA methyltransferase activity (IEA) \\
\hline orf19.3869 & 0.9 & 0.8 & 0.4 & 0.5 & 0.8 & 0.7 & 0.6 & 0.5 & \\
\hline orf19.5626 & 0.8 & 0.8 & 0.4 & 0.5 & 0.9 & 0.8 & 0.6 & 0.6 & \\
\hline orf19.28 & 0.8 & 0.7 & 0.5 & 0.6 & 1.0 & 0.9 & 0.6 & 0.6 & thiamin transmembrane transporter activity (IEA) \\
\hline orf19.4889 & 1.0 & 0.7 & 0.6 & 0.4 & 0.9 & 0.8 & 0.6 & 0.6 & drug transporter activity (ISS) \\
\hline orf19.3854 & 1.1 & 0.9 & 0.6 & 0.5 & 1.1 & 0.9 & 0.6 & 0.6 & protein kinase activity (IEA) \\
\hline orf19.2022 & 0.8 & 0.8 & 0.5 & 0.5 & 0.7 & 0.7 & 0.6 & 0.6 & glucose transmembrane transporter activity (ISS) \\
\hline
\end{tabular}


Appendix C. Continued.

\begin{tabular}{|c|c|c|c|c|c|c|c|c|c|c|}
\hline & & \multicolumn{8}{|c|}{ Change in fold expression ${ }^{a}$} & \\
\hline & & \multicolumn{2}{|c|}{$\underline{\text { G648S }}$} & \multicolumn{2}{|c|}{ G648D } & \multicolumn{2}{|c|}{$\underline{\mathrm{Y} 642 \mathrm{~F}}$} & \multicolumn{2}{|c|}{$\underline{\mathrm{A} 643 \mathrm{~V}}$} & \\
\hline \multicolumn{11}{|c|}{ CGD Name orf19 Designation Exp1 Exp2 Exp1 Exp2 Exp1 Exp2 Exp1 Exp2 Molecular function } \\
\hline & orf19.6691 & 0.9 & 0.8 & 0.5 & 0.4 & 0.8 & 0.9 & 0.6 & 0.6 & \\
\hline & orf19.4282 & 1.3 & 0.9 & 0.5 & 0.6 & 0.8 & 0.9 & 0.6 & 0.6 & \\
\hline & orf19.4959 & 0.5 & 0.9 & 0.4 & 0.6 & 0.5 & 0.9 & 0.5 & 0.6 & \\
\hline & orf19.3110 & 0.6 & 0.8 & 0.6 & 0.5 & 0.6 & 0.7 & 0.5 & 0.6 & \\
\hline & orf19.2726 & 0.8 & 1.2 & 0.6 & 0.5 & 0.7 & 0.9 & 0.6 & 0.6 & \\
\hline & orf19.6245 & 0.6 & 1.0 & 0.6 & 0.6 & 0.7 & 0.7 & 0.6 & 0.6 & \\
\hline & orf19.6077 & 0.8 & 0.9 & 0.6 & 0.5 & 0.6 & 0.7 & 0.6 & 0.6 & \\
\hline $\mathrm{MOHI}$ & orf19.3369 & 0.4 & 2.1 & 0.8 & 0.3 & 0.4 & 0.4 & 0.4 & 0.4 & \\
\hline \multirow[t]{4}{*}{$G A C 1$} & orf19.7053 & 0.7 & 1.9 & 1.2 & 0.7 & 0.6 & 0.6 & 0.6 & 0.6 & protein phosphatase type 1 regulator activity (IEA) \\
\hline & orf19.251 & 0.6 & 1.4 & 0.8 & 0.4 & 0.5 & 0.6 & 0.6 & 0.6 & \\
\hline & orf19.7311 & 0.6 & 1.0 & 0.7 & 0.8 & 0.6 & 0.6 & 0.6 & 0.6 & \\
\hline & orf19.1368 & 0.7 & 1.0 & 0.7 & 0.4 & 0.6 & 0.6 & 0.6 & 0.6 & \\
\hline$A O X 1$ & orf19.4774 & 0.5 & 0.8 & 0.4 & 0.3 & 0.4 & 0.4 & 0.3 & 0.3 & alternative oxidase activity (IMP, ISS) \\
\hline$A S R 2$ & orf19.7284 & 0.4 & 1.2 & 0.6 & 0.4 & 0.4 & 0.6 & 0.4 & 0.4 & \\
\hline$C D G 1$ & orf19.7314 & 1.2 & 0.6 & 0.6 & 0.5 & 0.6 & 0.5 & 0.6 & 0.5 & \\
\hline$C F L 2$ & orf19.1264 & 0.7 & 0.3 & 0.3 & 0.2 & 0.5 & 0.4 & 0.5 & 0.4 & ferric-chelate reductase activity (IEA) \\
\hline$D A L 8$ & orf19.5859 & 0.7 & 0.8 & 0.6 & 0.6 & 0.5 & 0.5 & 0.5 & 0.4 & anion:cation symporter activity (ISS) \\
\hline FMP45 & orf19.6489 & 0.4 & 1.7 & 0.5 & 0.4 & 0.2 & 0.3 & 0.3 & 0.5 & \\
\hline FRE10 & orf19.1415 & 1.0 & 0.6 & 0.4 & 0.4 & 0.6 & 0.4 & 0.5 & 0.4 & ferric-chelate reductase activity (IDA, IGI, IMP) \\
\hline HSP31 & orf19.3664 & 0.7 & 0.7 & 0.2 & 0.3 & 0.4 & 0.5 & 0.3 & 0.2 & \\
\hline RTA4 & orf19.6595 & 0.9 & 0.7 & 0.3 & 0.3 & 0.5 & 0.5 & 0.5 & 0.5 & phospholipid-translocating ATPase activity (IEA) \\
\hline \multirow[t]{2}{*}{ SOU1 } & orf19.2896 & 0.5 & 1.3 & 0.5 & 0.4 & 0.4 & 0.6 & 0.5 & 0.6 & mannitol 2-dehydrogenase (NADP+) activity (IDA) \\
\hline & orf19.3107 & 0.2 & 0.9 & 0.6 & 0.0 & 0.4 & 0.5 & 0.1 & 0.4 & \\
\hline
\end{tabular}


Appendix C. Continued.

\begin{tabular}{|c|c|c|c|c|c|c|c|c|c|c|}
\hline & & \multicolumn{8}{|c|}{ Change in fold expression ${ }^{a}$} & \\
\hline & & \multicolumn{2}{|c|}{$\underline{\text { G648S }}$} & \multicolumn{2}{|c|}{$\underline{\text { G648D }}$} & \multicolumn{2}{|c|}{$\underline{\mathbf{Y} 642 \mathrm{~F}}$} & \multicolumn{2}{|c|}{$\underline{\mathrm{A} 643 \mathrm{~V}}$} & \\
\hline \multicolumn{11}{|c|}{ CGD Name orf19 Designation Exp1 Exp2 Exp1 Exp2 Exp1 Exp2 Exp1 Exp2 Molecular function } \\
\hline & orf19.7310 & 0.4 & 1.5 & 0.6 & 0.3 & 0.4 & 0.5 & 0.5 & 0.4 & \\
\hline & orf19.822 & 0.3 & 1.4 & 0.3 & 0.3 & 0.4 & 0.5 & 0.3 & 0.4 & \\
\hline & orf19.3439 & 0.6 & 1.0 & 0.1 & 0.2 & 0.5 & 0.5 & 0.4 & 0.5 & \\
\hline & orf19.6741 & 0.7 & 0.8 & 0.4 & 0.4 & 0.5 & 0.6 & 0.4 & 0.5 & \\
\hline & orf19.1368 & 0.5 & 0.8 & 0.6 & 0.4 & 0.5 & 0.5 & 0.5 & 0.5 & \\
\hline & orf19.2846 & 0.5 & 1.9 & 0.5 & 0.3 & 0.5 & 0.5 & 0.5 & 0.6 & \\
\hline & orf19.3932 & 0.4 & 1.7 & 0.5 & 0.6 & 0.4 & 0.5 & 0.4 & 0.6 & \\
\hline & orf19.94 & 0.5 & 0.7 & 0.4 & 0.4 & 0.5 & 0.5 & 0.4 & 0.6 & \\
\hline$H A L 21$ & orf19.99 & 0.4 & 0.4 & 0.4 & 1.2 & 0.2 & 0.4 & 0.4 & 0.6 & 3'(2'),5'-bisphosphate nucleotidase activity (IEA) \\
\hline \multirow[t]{2}{*}{ SUL2 } & orf19.2738 & 0.6 & 0.6 & 0.2 & 0.3 & 0.4 & 0.5 & 0.7 & 0.7 & sulfate transmembrane transporter activity (NAS) \\
\hline & orf19.2163 & 0.3 & 0.5 & 0.5 & 0.6 & 0.4 & 0.4 & 0.9 & 1.0 & peptidase activity \\
\hline$H G T 10$ & orf19.5753 & 0.6 & 0.5 & 0.2 & 0.2 & 0.5 & 0.6 & 0.2 & 0.3 & glucose transmembrane transporter activity (ISS) \\
\hline \multirow[t]{4}{*}{ SAP7 } & orf19.756 & 0.4 & 0.4 & 0.3 & 0.2 & 0.4 & 0.4 & 0.3 & 0.4 & aspartic-type endopeptidase activity (IDA, ISS) \\
\hline & orf19.716 & 0.4 & 0.5 & 0.4 & 0.4 & 0.6 & 0.6 & 0.4 & 0.4 & \\
\hline & orf19.3621 & 0.3 & 0.5 & 0.3 & 0.3 & 0.6 & 0.4 & 0.5 & 0.5 & \\
\hline & orf19.4873 & 0.4 & 0.5 & 0.6 & 0.5 & 0.6 & 0.6 & 0.6 & 0.6 & \\
\hline
\end{tabular}

${ }^{a}$ Fold change defined as the average ratio of gene expression levels in the isolates compared in two independent microarray experiments Bold indicates real result as defined in Materials and Methods

Green shading indicates ratio values of 0.6 or less. 


\section{VITA}

Stephanie A. Flowers was born in 1978 in Oceanside, California, to Allan and Marcia Flowers. She graduated from Fallbrook Union High School in 1996 and then attended the University of California at Davis, graduating with a BS in Neurobiology, Physiology and Behavioral Sciences and a minor in Japanese. In 2003, she enrolled in pharmacy school at the University of Southern California where she graduated with honors and moved to Memphis, Tennessee, to complete her Pharmacy Practice Residency at the Veterans Affairs Medical Center in Memphis. After residency, she started her fellowship and graduate training at the University of Tennessee, Health Science Center and anticipates completing her Doctor of Philosophy in December of 2013. 